UNIVERSIDADE DE SÃO PAULO

INSTITUTO DE QUÍMICA

\title{
UM SISTEMA ESPECIALISTA PARA DETERMINAÇÃO ESTRUTURAL DE SESQUITERPENOS COM BASE EM DADOS DE RMN ${ }^{13} \mathrm{C}$
}

Francimeiry Cavalcante de Oliveira

Dissertação de Mestrado

Prof. Dr. Vicente de Paulo Emerenciano

Orientador

São Paulo

1998 


\section{"Um Sistema Especialista para Determinação Estrutural de Sesquiterpenos com Base em Dados de RMN ${ }^{13} \mathrm{C} "$.}

\section{FRANCIMEIRY CAVALCANTE DE OLIVEIRA}

Dissertação de Mestrado submetida ao Instituto de Química da Universidade de São Paulo como parte dos requisitos necessários à obtenção do grau de Mestre em Ciências - Área: Química Orgânica.

Aprovada por:

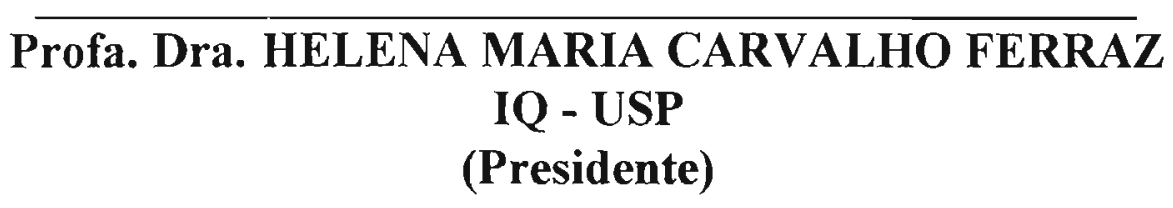

Profa. Dra. DAISY DE BRITO REZENDE

IQ - USP

Prof. Dr. GILBERTO DO VALE RODRIGUES

DQ - UFMG

SÃo PAULO

04 DE DEZEMBRO DE 1998 
Àquele que de diversas formas sempre está presente; À minha família, Josué (in memorian), Zilda e Silvia pelo amor incondicional, incentivo e apoio constante. 


\section{AGRADECIMENTOS}

Ao Prof. Dr. Vicente de Paulo Emerenciano por conceder-me a oportunidade de participar do seu grupo de pesquisa e desenvolver este trabalho.

Aos Profs. Drs. Jean Pierre Gastmans e Gilberto do Vale Rodrigues pela criação e aperfeiçoamento dos programas do SISTEMAT, que embasaram todo este trabalho.

Ao Prof. Dr. Júlio Militão pela indicação ao Prof. Vicente Emerenciano e pelo incentivo.

Aos amigos de PVh, Elcimar, Angelina e Jorjão pelo apoio imprescindivel e pela confiança.

Aos amigos de Sampa, Mara e Martin (desculpem-me aturá-los!), Kennia, Marcelo Silveira e Marcelo Pena por me ajudarem na digitação desta tese, por darem de si mesmos e por terem me prorporcionado tantos momentos bons. Conhecê-los foi saber o melhor!

Especialmente, ao amigo Marcelo Pena, pela paciência ao me ensinar o funcionamento do SISTEMAT, pelas suas sugestões, atenção e amizade. Haja mil dólares! Eternamente, obrigado!

Às amigas Juliana, Fátima, Patrícia, Cristina e Tanmatra por compartilharmos momentos felizes e momentos difíceis de nossa vidas.

Ao Toledo, pela ajuda inestimável no levantamento bibliográfico.

A todos os colegas do Bloco 11.

Ao Governo do Estado de Rondônia pela licença concedida.

À Capes, pela bolsa concedida. 


\section{THE ABSOLUTE}

No mind, no form, I only exist;

Now ceased all will and thought.

The final end of Nature's dance,

I am It whom I have sought.

A realm of Bliss bare, ultimate;

Beyond both knower and known;

A rest immense I enjoy at last;

I face the One alone.

I have crossed the secret ways of life;

I have become the Goal.

The Truth immutable is revealed;

I am the way, the God-Soul.

My spirit aware of all the heights,

I am mute in the core of the Sun.

I barter nothing with time and deeds;

My cosmic play is done.

(Sri Chinmoy) 


\section{RESUMO}

Este trabalho descreve a utilização dos programas do sistema especialista denominado SISTEMAT, avaliando a sua eficiência para auxiliar os pesquisadores da área de Produtos Naturais no processo de determinação estrutural de sesquiterpenos, a partir de dados de $\mathrm{RMN}{ }^{13} \mathrm{C}$.

Os principais sistemas especialistas em determinação estrutural operam, basicamente, combinando fragmentos estruturais, chamados "restrições estruturais", obtidos a partir de dados espectroscópicos. Ocorre que para moléculas complexas (com mais do que 15 átomos), o número de propostas estruturais geradas é muito grande, sendo necessária a ajuda do químico para eliminar estruturas improváveis. Este fato demonstra a necessidade de grandes restrições estruturais para o bom funcionamento do programa gerador, como por exemplo, a definição de classe e esqueleto, que foram utilizadas no desenvolvimento do SISTEMAT.

O SISTEMAT foi criado especificamente para determinação estrutural de substâncias de origem natural, sendo desenvolvidas técnicas para obtenção de regras heurísticas, a partir de dados de $\mathrm{RMN}^{13} \mathrm{C}$, que permitam a identificação de esqueletos de produtos naturais. Estes serão usados como grandes restrições pelo gerador de estruturas, evitando-se, assim, a explosão combinatória e a geração de propostas estruturais incompativeis.

Para a realização deste trabalho foi criado um banco de dados contendo os deslocamentos químicos e multiplicidades de 2306 espectros de sesquiterpenos, distrubuídos entre substâncias pertencentes a 288 tipos de esqueletos. Estes dados são analisados pelos vários programas aplicativos do SISTEMAT, sendo fornecidos os esqueletos mais prováveis para uma substância-problema e grandes subestruturas compativeis com o espectro analisado. Para avaliar o desempenho do sistema foram realizados testes com dados de $\mathrm{RMN}{ }^{13} \mathrm{C}$ de 60 sesquiterpenos.

A aplicação do SISTEMAT na identificação de sesquiterpenos em misturas, como por exemplo, em óleos essenciais, também foi investigada pela primeira vez, com resultados promissores. 


\section{ABSTRACT}

The present work describes the use of the expert system programs called SISTEMAT, evaluating their efficacy at helping Natural Products researchers to determine the structure of sesquiterpenes, using ${ }^{13} \mathrm{C}-\mathrm{NMR}$ spectroscopy data.

The major expert systems for structural determination operate by combining structural fragments called "structural restrictions", obtained from spectroscopic data. For complex molecules (bearing more than 15 atoms), the number of proposed structures becomes too large, hence the imposition of external chemical knowledge is required, in order to exclude improbable structures. This fact demonstrates the need of large structural restrictions to be imposed to get acceptable results output by the generator program. The definition of class and skeleton, which were used during the development of SISTEMAT, are examples of such restrictions.

SISTEMAT was created specially for natural products structural determination and methods were developed to obtain heuristic rules, mainly from ${ }^{13} \mathrm{C}-\mathrm{NMR}$ data, which allow the identification of natural products skeletal types. These will be used as large restrictions by the generator of structures, in order to avoid a combinatory explosion process at the generation step of the system.

To reach the purpose of this work a database was created with $2306{ }^{13} \mathrm{C}-\mathrm{NMR}$ spectra of sesquiterpenes, distributed in 288 skeletal types. Those data are used by the set of SISTEMAT programs which propose the most probable skeleton for the unknown sesquiterpene together with some large substructures compatible with the analysed spectrum. To evaluate the efficacy of the system, tests were carried out with the RMN ${ }^{13} \mathrm{C}$ data from 60 sesquiterpenes.

The application of SISTEMAT in the identification of sesquiterpenes in neat essential oils was also investigated for the first time with promising results. 


\section{CONTEÚDO}

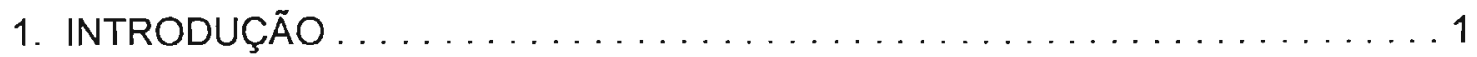

2. SISTEMAS ESPECIALISTAS EM DETERMINAÇÃO ESTRUTURAL

2.1. O Sistema DENDRAL .............................. 3

2.2. O Sistema Speclnfo .............................. 6

2.3. O Sistema especialista SISTEMAT

2.3.1. Breve histórico . . . . . . . . . . . . . . . . . . . . 11

2.3.2. Filosofia do SISTEMAT . . . . . . . . . . . . . . . . . . . 12

2.3.3. Codificação da estrutura. . . . . . . . . . . . . . . . . . . . 13

3. METODOLOGIA

3.1. O Levantamento bibliográfico. ........................ 18

3.2. A construção do banco de dados $\ldots \ldots \ldots \ldots \ldots \ldots \ldots \ldots \ldots \ldots \ldots$

3.3. Programas para a criação de bancos de dados ................. 36

3.4. PROGRAMAS APLICATIVOS . . . . . . . . . . . . . . . . . 40

3.4.1. Os programas SISLIST e REFERENC. . . . . . . . . . . . . . . . 40

3.4.2. Os programas SISBOTA e SISOCBOT . . . . . . . . . . . . . . . 46

3.4.3. O programa SISCONST . . . . . . . . . . . . . . . . . 48

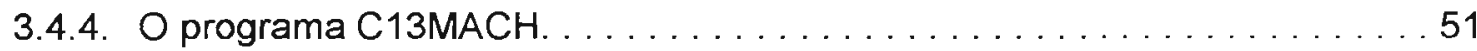

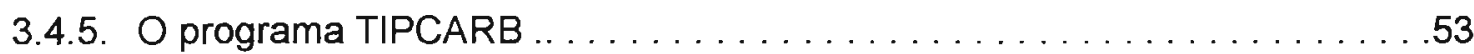

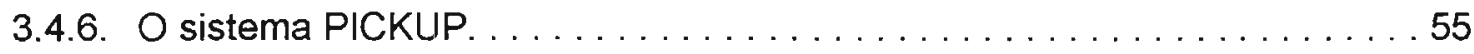

3.4.7. O sistema SESQREG.......................... 58

3.4.8. O programa MACRONO . . . . . . . . . . . . . . . . . . . . . . 67

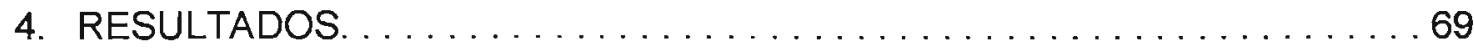

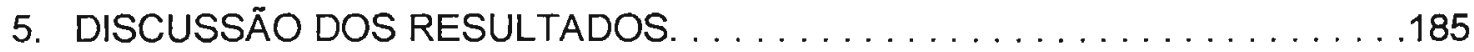

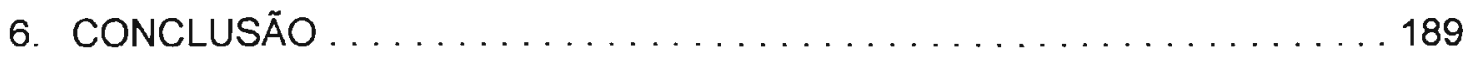

7. REFERÊNCIAS BIBLIOGRÁFICAS . . . . . . . . . . . . . . 191 


\section{ÍNDICE DE FIGURAS}

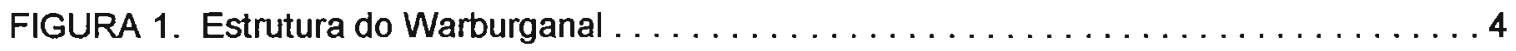

FIGURA 2. Fragmentos formecidos ao DENDRAL para gerar a estrutura do Warburganal. . . . 5

FIGURA 3. Fluxograma do SpecSolv $\ldots \ldots \ldots \ldots \ldots \ldots \ldots \ldots \ldots \ldots \ldots \ldots$

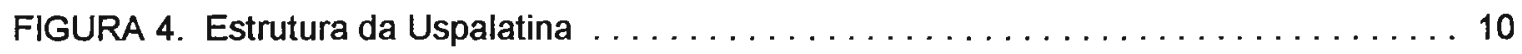

FIGURA 5. Estrutura da $23,24-$ Dihidro-15-oxocurcubitacina $F \ldots \ldots \ldots \ldots \ldots \ldots \ldots$

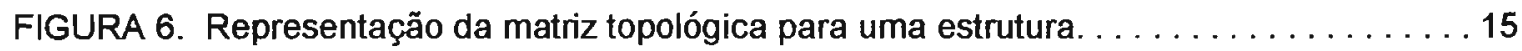

FIGURA 7. Exemplo utilizado para obtenção dos vetores $\ldots \ldots \ldots \ldots \ldots \ldots \ldots \ldots$

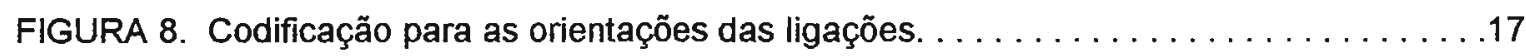

FIGURA 9. Esqueletos dos sesquiterpenos presentes no banco de dados. . . . . . . . 19

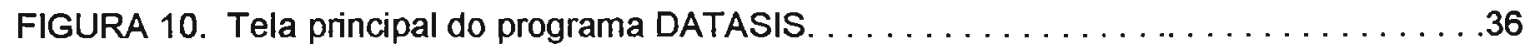

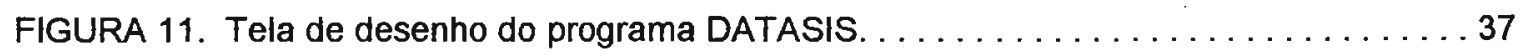

FIGURA 12. Tela de codificação do programa DATASIS $\ldots \ldots \ldots \ldots \ldots \ldots \ldots \ldots$

FIGURA 13. Esquema dos bancos arquiteturados do SISTEMAT . . . . . . . . . . 39

FIGURA 14. Estrutura do sesquiterpeno usado para teste do programa SISCONST. . . . . 49

FIGURA 15. Subestruturas propostas pelo programa SISCONST para a substância da figura

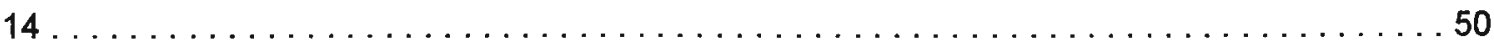

FIGURA 16. Sesquiterpenos com os melhores índices de similaridade . . . . . . . . .44

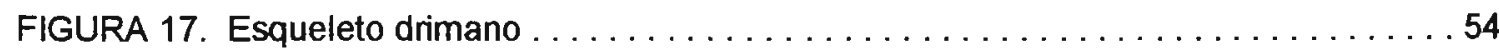

FIGURA18. Subestrutura inferida a partir do programa TIPCARB para obtenção de deslocamentos químicos característicos de drimanos . . . . . . . . . . . . . 57

FIGURA 19. Dados utilizados para testar o programa MACRONO $\ldots \ldots \ldots \ldots \ldots \ldots$ 


\section{ÍNDICE DE TABELAS}

TABELA 1. Tipos de átomos e ligações utilizadas no SISTEMAT.

TABELA 2. Listagem dos esqueletos presentes no banco de dados e o número de ocorrências .

TABELA 3. Listagem das ocorrências botânicas obtidas pelo programa Sislist para a família

Celastraceae

TABELA 4. Esqueletos de sesquiterpenos mais frequentes em 8 familias.

TABELA 5. Análise do esqueleto mais provável para uma dada substância, utilizando-se o programa SISCONST

TABELA 6. Dados de RMN ${ }^{13} \mathrm{C}$ do sesquiterpeno da FIGURA 14 associados aos deslocamentos químicos atribuídos às subestruturas $A$ e $B$ pelo programa SISCONST. . . . . . . . . 50

TABELA 7. Análise dos esqueletos mais prováveis fornecidos pelo programa $\mathrm{C} 13 \mathrm{MACH}$. . .52

TABELA 8. Listagem fornecida pelo programa TIPCARB para o esqueleto drimano 53

TABELA 9. Códigos das subestuturas utilizadas pelo programa PICKUP $\ldots \ldots \ldots \ldots \ldots$

TABELA 10. Códigos que representam as letras nas subestruturas da TABELA 9. .56

TABELA 11. Dados utilizados para desfuncionalização do espectro questionado 60

TABELA 12. Relação dos números de carbonos quaternários, metínicos, metilênicos e metílicos e o número total de carbonos dos esqueletos de sesquiterpenos presentes no banco de dados... .61

TABELA 13. Resultados dos testes com o programa MACRONO .60

TABELA 14. Resultados dos testes com o programa MACRONO, após a retirada dos sinais dos

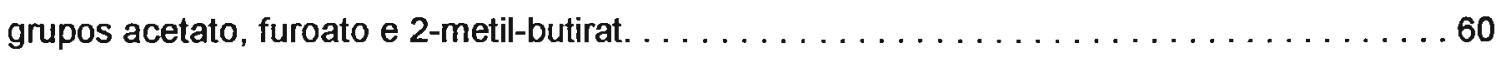

TABELA 15. Faixas de deslocamentos químicos característicos de subesqueletos de sesquiterpenos e respectivos percentuais de reconhecimento. . . . . . . . . . . 72 


\section{ABREVIATURAS}

EM - Espectro de Massas

IV - Infravermelho

RMN ${ }^{13} \mathrm{C}$ - Ressonância Magnética Nuclear de ${ }^{13} \mathrm{C}$

RMN ${ }^{1} \mathrm{H}$ - Ressonância Magnética Nuclear de ${ }^{1} \mathrm{H}$

IS - índice de similaridade

$s$ - singleto

d-dubleto

$t$ - tripleto

$q$ - quarteto

EP - epóxido

OXI - éter cíclico diferente de epóxido

OXO - carbonila

EN - dupla ligação

IN - tripla ligação

Ang - angeloil

Gly-glicose

$\mathrm{Xyl}$ - xilose

$p$ - para 


\section{INTRODUÇÃO}

O desenvolvimento acelerado dos computadores a partir da década de 50 , despertou o interesse de cientistas de diversas áreas do conhecimento, que pretendiam usá-los como ferramentas para auxiliar em suas pesquisas. Desde modo, surgiu, no início dos anos 60 , a idéia de se utilizar o computador para realizar tarefas que até então, eram consideradas possiveis apenas ao ser humano. Tarefas tais como a compreensão da linguagem natural, a prova de teoremas, o diagnóstico de doenças, a determinação estrutural e o planejamento de sínteses orgânicas, que requerem "inteligência". Começou a nascer, então, um novo ramo do conhecimento, chamado de Inteligênica Artificial, que procura simular o raciocínio humano, usando técnicas computacionais, para resolver problemas complexos. Nesta área, os primeiros problemas a serem estudados foram os jogos e a prova de teoremas ${ }^{1}$.

As pesquisas no campo da Inteligência Artificial têm obtido considerável sucesso no desenvolvimento de sistemas computacionais conhecidos como Sistemas Especialistas ${ }^{2}$, porque solucionam problemas de domínio restrito, usando um modelo computacional do raciocinio de um especialista humano. Estes sistemas, embora atuem em diferentes áreas, apresentam alguns aspectos em comum como, por exemplo, uma grande base de conhecimento, um processo de representação adequada deste conhecimento para o próprio sistema, flexibilidade para aquisição de novos conhecimentos e um grau de confiabilidade da resposta fornecida. Além disso, devem interagir facilmente com o usuário.

Quando os Sistemas Especialistas realizam buscas exaustivas e sistemáticas, que muitas vezes resultam numa explosão combinatorial ou em tempo computacional muito alto, é necessário uma redução do espaço de busca, ou espaço de soluções. Para isto, utilizam-se as chamadas regras heurísticas, que são regras informais e podem ser entendidas como uma resposta pronta, desenvolvida na mente do especialista após longa observação de resultados típicos ${ }^{2}$. Estas regras dão uma orientação sobre como resolver um problema particular. Por exemplo, a afirmação de que "muitas drogas são moléculas de baixo peso molecular" é uma heurística. Ela não define como sintetizar uma droga útil, ou que estrutura ela deve ter, mas ela ajuda a reduzir o espaço de busca ${ }^{3}$, encurtando os caminhos para se chegar a uma solução, embora nem sempre garantam que seja encontrada a solução perfeita. 
O domínio dos sistemas especialistas está se expandindo rapidamente, demonstrando suas conveniências para muitos setores da economia, particularmente no campo de projetos tecnológicos e científicos complexos (por exemplo, o controle da missão espacial na NASA, explorações geológicas, automação de produção industrial e outros) ${ }^{4}$.

Na Medicina, destaca-se o sistema $\mathrm{MYCIN}^{5}$, desenvolvido na Universidade de Stanford, que realiza o diagnóstico de doenças infecciosas bacterianas, possuindo regras heurísticas que incluem sintomas, diagnósticos, sugestôes de exames adicionais e terapias sugeridas, informando os medicamentos e melhor tratamento a serem utilizados ${ }^{5}$. Na área de Geologia, o programa PROSPECTOR ${ }^{6}$ fornece assessoria na prospecção de petróleo e minerais, tendo descoberto um depósito de molibdênio cujo valor excedeu 100 milhões de dólares. Na Química, podemos destacar, na área de síntese orgânica, o programa $\mathrm{CAMEO}^{7}$, que faz a previsão lógica dos produtos de reações orgânicas e, na área de determinação estrutural, os programas DENDRAL ${ }^{8}$, DARC/EPIOS $^{9}$ e Speclnfo ${ }^{10}$.

Uma das grandes vantagens dos sistemas especialistas é a disseminação do conhecimento humano especializado, raro e de alto custo, além da possibilidade de se combinar o conhecimento de muitos especialistas em um banco de dados. Com o advento dos microcomputadores pessoais e dos terminais de trabalho, a criação destes sistemas tornou-se financeiramente viável.

A seguir, serão descritos os sistemas DENDRAL, que foi um sistema pioneiro na área de determinação estrutural, o Speclnfo, um dos sistemas mais recentes e o SISTEMAT, que é o objeto de estudo deste trabalho. 


\section{SISTEMAS ESPECIALISTAS EM DETERMINAÇÃO ESTRUTURAL}

Nos últimos anos, a aquisição de dados espctroscópicos tem tornado-se cada vez mais fácil, devido ao uso de computadores no controle dos espectrômetros modernos e ao uso de trocadores de amostra automático. No entanto, a eficiência de um laboratório de espectroscopia é determinada pela velocidade com a qual ele dá respostas para as questões analíticas e não apenas pela rapidez na geração de dados. Portanto, a interpretação de dados é um ponto fundamental em laboratórios que trabalham com elucidação estrutural.

Em algumas indústrias, por exemplo, o espectroscopista desempenha um papel chave em muitos estágios do desenvolvimento de um novo produto, devendo fornecer informações rápidas e confiáveis que serão a base para a tomada de muitas decisões. O período de tempo que uma inovação feita no laboratório leva para atingir o mercado determinará o sucesso ou fracasso de um produto, ou mesmo, de uma companhia inteira. Para vencer esta limitação, têm sido desenvolvidas, nos últimos 30 anos, várias abordagens para criação de sistemas especialistas em determinação estrutural.

\subsection{O sistema DENDRAL}

O sistema DENDRAL ${ }^{8}$, amplamente citado como o primeiro sistema especialista completo usado rotineiramente pelos químicos orgânicos, resultou em mais de 50 artigos publicados e teve sua origem no início dos anos 60 , na Universidade de Stanford, quando o prof. Lederberg, interessado pelo problema do isomerismo químico, criou um sistema que era um algoritmo combinatorial para gerar todas as estruturas possíveis de uma dada composição química ${ }^{11}$.

O DENDRAL utiliza dados de várias técnicas espectrométricas, entre elas a espectrometria de massas, RMN ${ }^{13} \mathrm{C}, \mathrm{RMN}{ }^{1} \mathrm{H}$ e IV, sendo desenvolvido para uso em computadores de grande porte. A metodologia usada por este sistema consiste no método básico "planejar-gerar-testar", que está relacionado com as fases tipicamente desenvolvidas no processo de elucidação estrutural feito pelo químico: a interpretação de dados, a geração de estrutura e a avaliação estrutural. 
Na fase de Planejamento, que é a etapa mais crítica do processo, o sistema requer uma grande interação com o usuário, pois ele é quem fornecerá ao sistema os fragmentos subestruturais inferidos a partir da ánalise de dados químicos e espectrais.

$\mathrm{Na}$ etapa de geração de estruturas, o módulo gerador, chamado GENOA, utiliza os dados fornecidos pelo usuário na fase anterior e gera todos os isômeros possíveis, ligando os fragmentos segundo as regras de valências. Este procedimento é exaustivo, de modo a garantir que todas as combinações serão feitas. Portanto, o número de soluções é muito grande quando são fornecidos muitos fragmentos. Nesta fase também há a participação do usuário. Os fragmentos maiores e as estruturas parciais geradas pelo programa são submetidos à apreciação do químico e este pode eliminá-las ou mantê-las para geração das propostas estruturais definitivas. Deste modo, eliminam-se subestruturas incompatíveis antes da geração exaustiva e economiza-se tempo.

A fase de teste consiste na avaliação das estruturas propostas para eliminação daquelas que forem improváveis. Isto pode ser feito através da simulação de espectros para as estruturas e comparação com os espectros questionados ou, caso a substância seja um produto natural, confrontando-se as estruturas com os bancos de esqueletos de produtos naturais.

Um dos exemplos importantes e bem descrito na literatura ${ }^{12}$ sobre a aplicação desse sistema é a elucidação estrutural do sesquiterpeno warbuganal (figura 1), isolado de Warburgia ugandensis ${ }^{13}$, com fórmula molecular $\mathrm{C}_{15} \mathrm{H}_{22} \mathrm{O}_{3}$ e um esqueleto do tipo drimano. Neste teste, o usuário fornece ao programa a fórmula molecular, obtida do espectro de massas, os fragmentos estruturais inferidos a partir dos dados espectrais de IV, RMN ${ }^{1} \mathrm{H}, \quad \mathrm{RMN}{ }^{13} \mathrm{C}$ e experimentos de dupla irradiação (desacoplamento) em RMN ${ }^{1} \mathrm{H}$, bem como o número de ocorrências destes e outros fragmentos que não podem existir na molécula, em virtude dos dados espectrais analisados (figura 2).

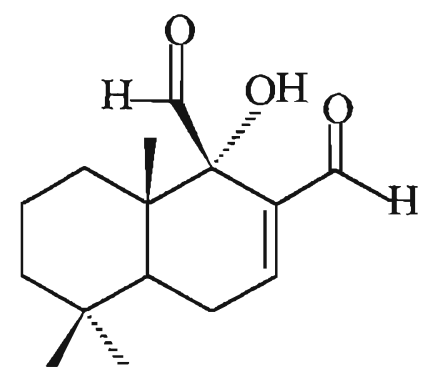

Figura 1. Estrutura do Warburganal 
FRAGMENTOS

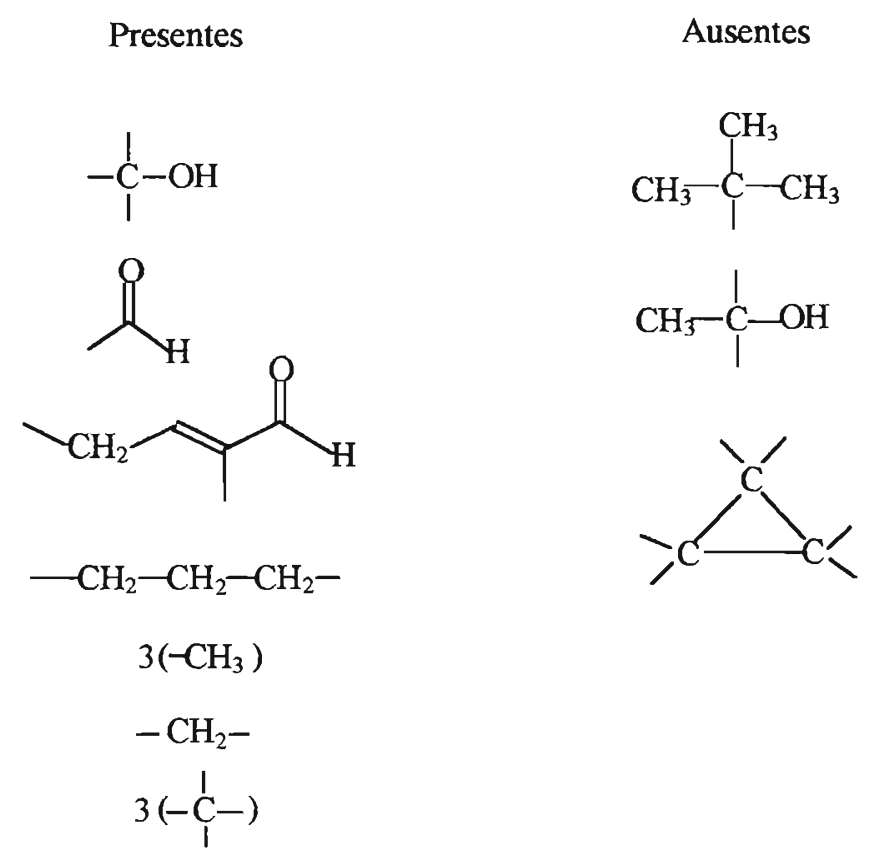

Figura 2. Fragmentos fornecidos ao DENDRAL para gerar a estrutura do Warburganal.

Com as restrições impostas ao programa foram geradas 42 estruturas, dentre elas, o warburganal. Muitas destas estruturas, no entanto, eram incompatíveis com produtos naturais, sendo descartadas na fase de teste. Nesta fase, é utilizado o módulo SURVEY, que confronta as estruturas propostas com um banco de esqueletos de produtos naturais e mostra os esqueletos ou fragmentos de esqueletos encontrados nas estruturas. Das 42 candidatas, apenas uma incorporava um esqueleto completo e correspondia à estrutura proposta para o warburganal. Uma outra forma de avaliação das estruturas propostas é a previsão dos espectros de RMN ${ }^{13} \mathrm{C}$ e EM e a comparação destes com o espectro real. Deste modo, podem-se eliminar substâncias naturais improváveis. 


\section{2- O sistema Speclnfo}

O sistema de informação espectroscópica multidimensional Specinfo tem sido desenvolvido pela BASF, desde 1970, como uma ferramenta para dar assistência ao espectroscopista analítico, que se depara com um fluxo constante de espectros e cujo serviço deve ser rápido, barato e confiável. Encontra-se, atualmente, em sua terceira versão e foi desenvolvido para rodar em estações de trabalho, utilizando uma interface gráfica do tipo $X$-Windows ${ }^{14}$.

O Speclnfo possui um sistema de codificação de subestruturas baseado na idéia de que ambientes químicos influenciam as propriedades de qualquer átomo em uma mólecula, utilizando o código HOSE (Hierarchically Ordered Spherical Description of Environment) ${ }^{15}$ para caracterizar o ambiente de átomos individuais esfericamente. Este código é basicamente uma sequência descrevendo os átomos que circundam um dado nó central, até a $3^{\text {a }}$ esfera. Deste modo, os efeitos sobre acoplamento e deslocamentos químicos do átomo central fazem parte da informação calculada e armazenada para qualquer átomo particular na base de dados. $O$ resultado deste conceito é um "estoque genético" de ambientes químicos de átomos, cuja aplicabilidade não está limitada a classes específicas de substâncias.

Como cada membro da base de dados está correlacionado com um padrão espectral, torna-se possivel estimar o espectro de uma dada estrutura química, a qual é convertida automaticamente em uma lista de códigos HOSE, um para cada átomo da molécula. No caso de RMN ${ }^{13} \mathrm{C}$, uma busca na biblioteca fornecerá um deslocamento químico para cada código HOSE desta lista.

A base de dados do Speclnfo é uma das maiores disponibilizadas e está sendo continuamente ampliada. Até 1990 , ela continha 110.000 espectros de $\mathrm{RMN}{ }^{13} \mathrm{C}$, 15.000 espectros de $R M N{ }^{31} \mathrm{P},{ }^{19} \mathrm{~F},{ }^{17} \mathrm{O},{ }^{15} \mathrm{~N},{ }^{11} \mathrm{~B}, 20.000$ espectros de IV e 16.000 espectros de massas. Para assegurar um excelente funcionamento das ferramentas de previsão de espectro dentro do Specinfo, cada espectro a ser incluído na base de dados passa por um procedimento de segurança de qualidade, que consiste em testes automatizados e uma inspeção final por um espectroscopista experiente, de modo a certificar-se de que espectros de baixa qualidade sejam identificados antes de contaminar a base de dados.

Recentemente, foi adicionado ao pacote de programas do Specinfo um novo módulo, denominado SpecSolv, que é considerado pelos autores uma ferramenta realmente inovadora para elucidação estrutural completamente automatizada ${ }^{16}$. Este 
sistema está baseado exclusivamente em $\mathrm{RMN}{ }^{13} \mathrm{C}$ e não requer informações adicionais de outras técnicas espectroscópicas, nem mesmo o conhecimento da fórmula molecular.

A elucidação estrutural no SpecSolv está dividida em três etapas:

1. A aquisição do espectro de $R M N^{13} \mathrm{C}$-DEPT experimental e a extração dos dados de deslocamentos químicos, intensidades e multiplicidades;

2. A busca de subespectros em uma biblioteca de correlações subestruturasubespectro (SSC) e geração de uma lista de resultados positivos;

3. A reunião das subestruturas, usando-se uma abordagem que tira vantagem da informação de subestruturas parcialmente ou totalmente sobreponiveis, seguida pelos passos de validação para estruturas intermediárias.

A base de conhecimento do SpecSolv consiste de uma base de dados de SCC, contendo mais de 400.000 subestruturas de códigos HOSE de três esferas e mais de 100.000 de códigos HOSE de duas esferas. Estes dados foram derivados da base de dados de Speclnfo que possui atualmente 200.000 espectros de RMN ${ }^{13} \mathrm{C}$. Um conjunto SSC contém as informações sobre o subespectro, a matriz de conectividade e o código HOSE para todos os átomos. Os parâmetros de $\mathrm{RMN}{ }^{13} \mathrm{C}$ já citados também são armazenados para cada átomo de cada subestrutura.

A seguir são descritas as etapas 2 e 3 do processo de elucidação estrutural no SpecSolv.

\section{A BUSCA DE SUBESPECTROS}

Esta etapa consiste na comparação de todos os subespectros da base de dados de SSC com o espectro questionado, sendo aceitas apenas as correlações subestrutura-subespectro (SSC) cujas diferenças de deslocamentos químicos, com relação ao espectro experimental, encontrem-se dentro da faixa definida pelo usuário, considerando-se também as intensidades e as multiplicidades.

As subestruturas selecionadas são então classificadas de acordo com um fator de comparação, que indica o desvio médio dos deslocamentos químicos entre as SSCs e o espectro questionado, em ppm. 


\section{A GERAÇÃO DE ESTRUTURAS}

Na descrição do código HOSE das SSC, cada átomo de uma subestrutura tem um código HOSE individual, consequentemente os átomos vizinhos têm algo redundante. Esta redundância na descrição estrutural é a chave para o novo processo de combinação de subestruturas no SpecSolv.

As subestruturas provenientes da busca de SSC são ligadas pela sobreposição dos átomos comuns a duas subestruturas. A subestrutura maior resultante tem sua plausibilidade testada através da previsão do seu espectro, antes de ser aceita e sobreposta a uma terceira subestrutura.

O processo de combinação começa com a subestrutura maior e com o melhor fator de comparação. Se não existir nenhuma sobreposição da subestrutura de partida com uma segunda subestrutura, o SpecSolv escolhe a próxima estrutura. Caso haja sobreposição, ambos os subespectros são combinados e o espectro resultante é comparado ao experimental. Se eles foram compatíveis, a tabela de conexão para uma estrutura maior é gerada e esta estrutura é usada como estrutura de partida. Deste modo, o processo se repete até que todas as subestruturas sejam sobrepostas com a estrutura de partida e/ou todos os deslocamentos químicos do espectro questionado estejam completamente atribuídos.

Durante o processo de combinação das subestruturas individuais, todos os deslocamentos químicos da subestrutura resultante ficam disponiveis na memória central do computador e um novo subespectro pode ser gerado por simples combinação dos subespectros originais. Isto torna o processo de validação de estrutura muito rápido porque não é necessário o acesso a uma base de dados e também permite a verificação de cada subestrutura intermediária, bem como da estrutura final, verificando-se se o espectro previsto é compativel ou não com o questionado. As subestruturas com desvios de deslocamento químico acima de uma faixa definida são rejeitadas. A figura 3 descreve um fluxograma simplificado do gerador de estrutura do SpecSolv.

A validação imediata de estruturas intermediárias é uma das principais diferenças entre o SpecSolv e outros programas de geração de estruturas, que a realizam após a geração da molécula inteira. Isto torna o espaço de solução significantemente menor e, por sua vez, conduz a uma redução crucial no tempo computacional.

As técnicas de sobreposição e a metodologia de previsão espectral adotados pelo Speclnfo funcionam bem para moléculas com massa até aproximadamente 
1000Da. Moléculas maiores podem requerer um tempo computacional de horas devido ao número combinatorial de possibilidades para sobrepor as estruturas.

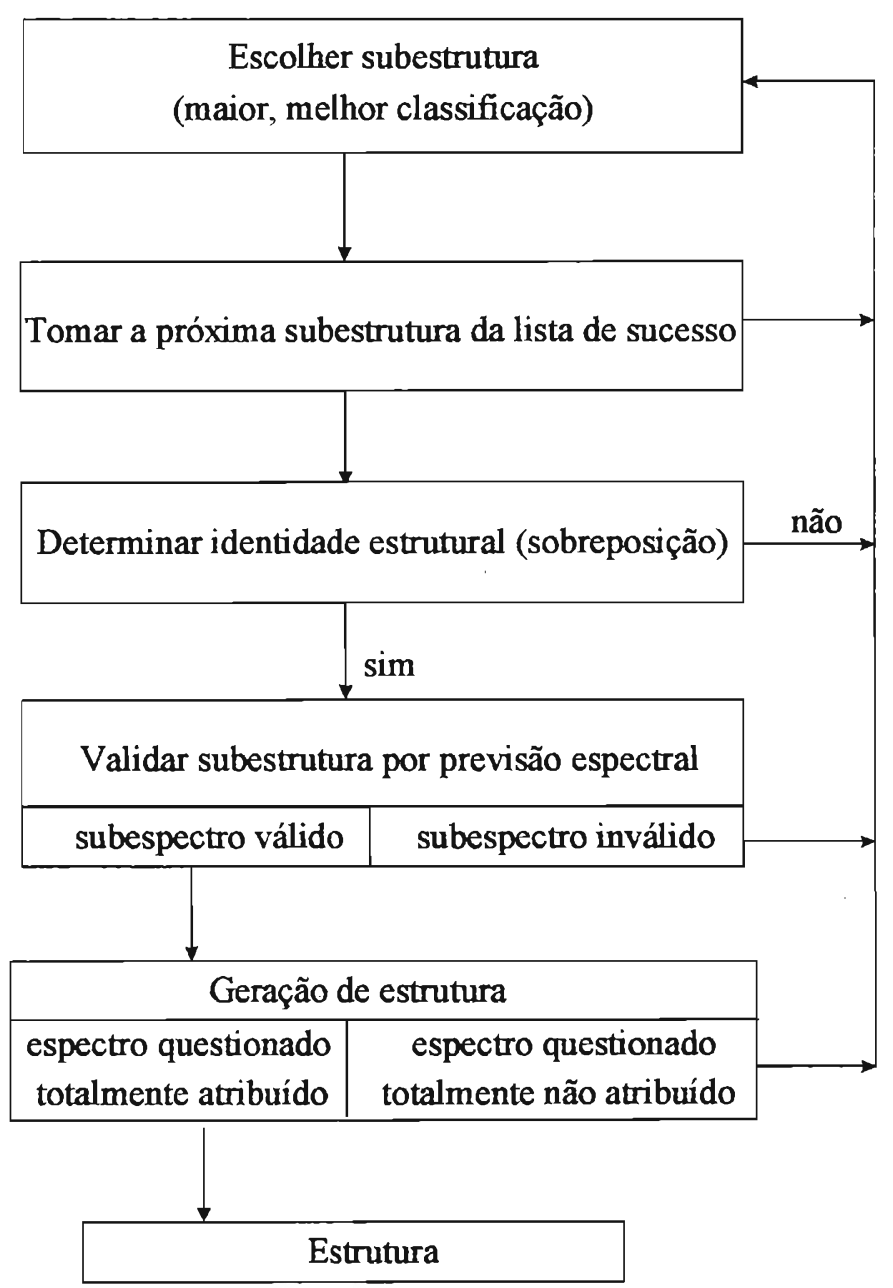

Figura 3. Fluxograma do SpecSolv.

Para demonstrar o funcionamento e o potencial do SpecSolv, foram realizados testes com as substâncias Uspalatina ${ }^{17}$ e 23,24-Di-hidro-15-oxocurcubitacina $F^{18}$ (figuras 4 e 5 , respectivamente).

A elucidação estrutural da Uspalatina foi realizada num tempo menor que 3 minutos, em um computador VAX 6610. Os dados de entrada requeridos pelo programa foram os deslocamentos químicos, multiplicidades e intensidades dos sinais, o espectro de $\mathrm{RMN}{ }^{13} \mathrm{C}$ e um teto máximo para fórmula molecular do tipo 
$\mathrm{C}_{100} \mathrm{H}_{100} \mathrm{O}_{100} \mathrm{~N}_{100}$. Neste caso, também foi feita uma estimativa do número máximo de átomos de nitrogênio próximo do valor real, para redução do tempo computacional. $A$ busca de subespectros resultou em 150 subestruturas, a partir das quais, o gerador construiu apenas uma molécula com espectro compatível com o experimental e um fator de comparação de 0,44 ppm, que é conhecida como Uspalatina. O espectro experimental completamente atribuído foi adicionado à base de dados do Speclnfo e à biblioteca de SSC do SpecSolv.<smiles>C/C=C1/CC(C)C(C)(O)C(=O)OCC2=CCN3CC(O)C(OC1=O)C23</smiles>

Figura 4. Estrutura da Uspalatina

Para o espectro da 23,24-Di-hidro-15-oxocurcubitacina $F$, a busca de subestruturas resultou em 1118 SSCs, porém o gerador forneceu apenas duas estruturas possíveis, em menos de 3 minutos, ambas com bons fatores de comparação, sendo uma delas descartada por conter um sistema cíclico com uma ponte não usual. A estrutura melhor classificada corresponde à substância da figura 5.

Vale ressaltar que este sistema só é capaz de elucidar estruturas que podem ser descritas por sobreposição de SSCs, sendo a qualidade e diversidade da base de dados de SSC fatores cruciais na determinação estrutural. Além disso, esta base de dados ainda não contém informações estereoquímicas, não levando em conta efeitos configuracionais.<smiles>CC(C)(O)CCC(=O)C(C)(O)C1C(O)C(=O)C2(C)C3CC=C4C(CC(O)C(O)C4(C)C)C3(C)C(=O)CC12C</smiles>

Figura 5. Estrutura da 23,24-Dihidro-15-oxocurcubitacina F. 


\section{3 - O sistema especialista SISTEMAT}

\subsection{1- BREVE HISTÓRICO}

O sistema especialista SISTEMAT teve sua origem em 1988, no Instituto de Química da USP, e foi desenvolvido pelos professores Emerenciano e Gastmans para auxiliar pesquisadores da área de Produtos Naturais no processo de determinação estrutural, bem como em estudos quimiotaxonômicos, sendo estes últimos ainda pouco explorados. Este sistema, atualmente, ainda se encontra em fase de desenvolvimento e aperfeiçoamento.

Inicialmente, foram desenvolvidas técnicas de codificação, ou seja, de representação de substâncias e de arquivamento dos dados relacionados a estas, como por exemplo, dados espectrais e botânicos ${ }^{19,20}$. Posteriormente, foram criados vários programas aplicativos para determinação estrutural ${ }^{21,22,23}$, utilizando-se dados de Espectrometria de Massas, RMN ${ }^{1} \mathrm{H}$ e, principalmente, de $\mathrm{RMN}{ }^{13} \mathrm{C}$, que são os mais explorados, tendo em vista o número de informações que se pode obter a partir dos espectros de RMN ${ }^{13} \mathrm{C}$. Também foram utilizados dados botânicos para a criação de alguns programas ${ }^{24,25}$, como filtros para orientar o químico quanto à possibilidade de se encontrar os principais tipos de esqueletos presentes em determinados taxons.

O processo de codificação de estruturas era, inicialmente, manual e a criação dos bancos de dados requeria vários programas, o que tornava este processo muito lento para ser utilizado. Em 1992, foi desenvolvido o programa $\operatorname{CODISIS}^{26}$ que permitia a codificação semi-automática, requerendo que o usuário estabelecesse apenas a sequência de numeração da cadeia carbônica, e substituia alguns dos programas de criação de banco de dados. Posteriormente, este programa foi aperfeiçoado e recebeu o nome de DATASIS ${ }^{25}$, tornando-se a codificação totalmente automática e ampliando-se os recursos de manutenção dos bancos de dados. Isto aumentou consideravelmente a velocidade de arquivamento de dados e diminuiu 0 número de erros que poderiam ocorrer na codificação manual, tornando favorável também a exploração do sistema para aspectos quimiotaxonômicos, tendo em vista que este tipo de estudo requer uma grande quantidade de informação.

O crescimento dos bancos de dados do SISTEMAT se dá por classes químicas, de modo a se ter um controle das substâncias já arquivadas e para que cada pessoa do grupo trabalhe com substâncias afins. 
Atualmente, o SISTEMAT conta com um total de 11.000 espectros de RMN ${ }^{13} \mathrm{C}$ e cerca de 1000 espectros de massas de produtos naturais, tendo sido estudadas as seguintes classes de substâncias naturais: monoterpenos ${ }^{27}$, iridóides ${ }^{27}$, sesquiterpenos lactonizados $^{28}$ e não lactonizados ${ }^{29}$, diterpenos ${ }^{24}$, triterpenos ${ }^{25}$, esteróides ${ }^{30}$, flavonóides $^{31}$ e neolignanas. Pretende-se, futuramente, acrescentar ao banco outras classes, tais como, alcalóides e cumarinas.

Os programas aplicativos já foram testados com sucesso em outros trabalhos e teses, sendo agora aplicados à base de dados de sesquiterpenos.

Recentemente, foi desenvolvido o programa gerador de estruturas do SISTEMAT, denominado SISGER ${ }^{32}$, que utiliza informações sobre o tipo de esqueleto do produto natural e fórmula molecular, começando o processo de geração com um determinado esqueleto carbônico inferido a partir de outros programas, descritos no item PROGRAMAS APLICATIVOS. Além do conceito de esqueleto, o gerador também utiliza um banco de dados de subestruturas com a descrição de carbonos em diversos ambientes químicos. Tais subestruturas são conhecidas como ELCOs ${ }^{33}$ (ambientes que são limitados, concêntricos e ordenados) e são gerados a partir dos bancos fonte do SISTEMAT. A geração de estruturas ocorre pela sobreposição progressiva dos ELCOs, respeitando, contudo, a condição do tipo de esqueleto.

\subsection{2- FILOSOFIA DO SISTEMAT}

Ao contrário de outros sistemas, o SISTEMAT tem sua aplicação totalmente voltada para a química de produtos naturais, onde a informação sobre a classe química da substância e o tipo de esqueleto carbônico são pontos-chave no processo de determinação estrutural, quer seja ele desempenhado por um especialista ou por programas computacionais. Sendo assim, foram introduzidos nos bancos de dados do SISTEMAT as informações sobre a classe química e o esqueleto de cada substância, e foram desenvolvidos programas que permitem a busca de faixas de deslocamentos químicos de $\mathrm{RMN}{ }^{13} \mathrm{C}$ característicos dos diversos tipos de esqueletos ou subesqueletos.

A utilização do conceito de esqueleto durante a etapa de geração de estrutura reduz grandemente a possibilidade de um número enorme de estruturas propostas, ou seja, de uma explosão combinatorial, o que normalmente ocorre em outros sistemas, quando se analisam moléculas muito complexas como os triterpenos, por exemplo. 
Além de resultar num menor tempo computacional e produzir um número menor de estruturas incompatíveis.

Uma das grandes vantagens do SISTEMAT com relação a outros sistemas especialistas é o fato dele possuir um sistema de codificação estrutural e de compactação de dados extremamente eficiente, permitindo assim a utilização de microcomputadores em ambiente DOS. Todas as informações possíveis acerca de todos os produtos naturais conhecidos não ocupariam mais de $35 \mathrm{Mb}$. Os outros sistemas são executados em computadores de grande e médio porte. Portanto, este é um sistema desenvolvido com um baixo custo e que pode ser utilizado por qualquer usuário que possua um microcomputador.

O método de codificação utilizado pelo SISTEMAT também permite que o próprio sistema descubra informações químicas das substâncias, tais como grupos funcionais, massa molecular, fórmula molecular e número de oxidação, através da fórmula estrutural codificada. Além disso, o SISTEMAT também apresenta flexibilidade para o armazenamento de dados espectrais de substâncias de origem natural, juntamente com a classe química e com informações botânicas (familia, gênero, espécie), utéis em estudos quimiotaxonômicos e evolutivos.

Os primeiros programas do SISTEMAT foram escritos em FORTRAN e os mais recentes em PASCAL.

Atualmente, o SISTEMAT apresenta, basicamente, dois tipos de programas: Os programas de criação de banco de dados e os programas aplicativos.

\subsection{3- CODIFICAÇÃO DA ESTRUTURA}

O passo preliminar na construção de qualquer sistema especialista na área de Química Orgânica é a representação de uma substância em uma linguagem matématica que possa ser entendida pelo computador. Tal processo é chamado de codificação molecular.

No método de codificação utilizado pelo SISTEMAT, a molécula é tratada como se fosse um grafo, ou seja, uma interligação entre pontos, chamados nós (átomos da molécula), através de vértices (ligações químicas). Como existem vários tipos de átomos e ligaçōes químicas, o grafo é chamado de ponderado, sendo atribuídos pesos puramente arbitrários aos tipos de átomos e ligações (tabela 1). 
Tabela 1 - Tipos de átomos e ligações utilizadas no SISTEMAT.

\begin{tabular}{l} 
ÁTOMO \\
\begin{tabular}{|l|c|c|c|}
\hline \multicolumn{4}{|c|}{ LIGAÇÃO } \\
\hline Carbono & PESO & TIPO & PESO \\
\hline Carbono Aromático & 1 & Nenhuma & 0 \\
\hline Oxigênio & 2 & Simples & 1 \\
\hline Nitrogênio & 3 & Aromática & 1 \\
\hline Nitrogênio Aromático & 4 & Dupla & 2 \\
\hline Flúor & 5 & Tripla & 3 \\
\hline Cloro & 6 & & \\
\hline Bromo & 7 & & \\
\hline lodo & 8 & & \\
\hline Enxofre & 9 & & \\
\hline Fósforo & 10 & & \\
\hline
\end{tabular} \\
\hline
\end{tabular}

Um dos recursos matemáticos mais simples para representação de um grafo é a matriz topológica, que é construída a partir do desenho da estrutura e numeração dos seus átomos. Os elementos da diagonal representam os pesos dos átomos na ordem em que foram numerados e, fora da diagonal, representam os tipos de ligações entre os átomos das coodenadas do elemento. Na figura 6 é dado um exemplo de como seria a matriz topológica da estrutura desenhada. Analisando esta matriz o computador pode encontrar as principais informações estruturais da molécula, faltando apenas a representação das ligações alfa e beta, ligações transanulares de conformação cis e os macronós (substituintes comuns em produtos naturais, tais como, angelato e acetato). Estes são codificados em quatro vetores distintos, por sua vez associados à matriz.

No entanto, o armazenamento de toda a matriz juntamente com os vetores ocuparia um grande volume de memória, fugindo do objetivo proposto na idealização do SISTEMAT, que é a maior compactação possivel de dados. Para solucionar este problema, fez-se uso da técnica do vetor reduzido, cuja aplicação só é possivel porque a maioria dos elementos da matriz topológica são nulos. Este vetor consiste numa sequência das posições e valores dos elementos não nulos da matriz.

Depois disso, ainda é necessário outro método de codificação que permita ao computador transformar os números da matriz numa linguagem familiar ao químico, 0 desenho da molécula. Foi então desenvolvido, por tentativa e erro, um método de codificação arbitrário, que conduzisse a um vetor com menor tamanho possivel, contendo todas as informaçōes necessárias. Este vetor foi denominado vetor nó. 


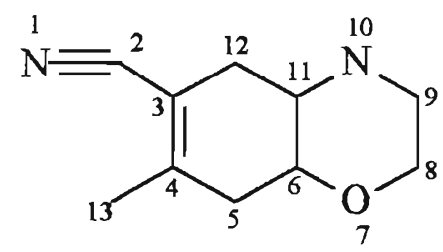

\begin{tabular}{|c|ccccccccccccc|}
\hline & 1 & 2 & 3 & 4 & 5 & 6 & 7 & 8 & 9 & 10 & 11 & 12 & 13 \\
\hline 1 & 4 & 3 & 0 & 0 & 0 & 0 & 0 & 0 & 0 & 0 & 0 & 0 & 0 \\
2 & 3 & 1 & 1 & 0 & 0 & 0 & 0 & 0 & 0 & 0 & 0 & 0 & 0 \\
3 & 0 & 1 & 1 & 2 & 0 & 0 & 0 & 0 & 0 & 0 & 0 & 1 & 0 \\
4 & 0 & 0 & 2 & 1 & 1 & 0 & 0 & 0 & 0 & 0 & 0 & 0 & 1 \\
5 & 0 & 0 & 0 & 1 & 1 & 1 & 0 & 0 & 0 & 0 & 0 & 0 & 0 \\
6 & 0 & 0 & 0 & 0 & 1 & 1 & 1 & 0 & 0 & 0 & 1 & 0 & 0 \\
7 & 0 & 0 & 0 & 0 & 0 & 1 & 3 & 1 & 0 & 0 & 0 & 0 & 0 \\
8 & 0 & 0 & 0 & 0 & 0 & 0 & 1 & 1 & 1 & 0 & 0 & 0 & 0 \\
9 & 0 & 0 & 0 & 0 & 0 & 0 & 0 & 1 & 1 & 1 & 0 & 0 & 0 \\
10 & 0 & 0 & 0 & 0 & 0 & 0 & 0 & 0 & 1 & 4 & 1 & 0 & 0 \\
11 & 0 & 0 & 0 & 0 & 0 & 1 & 0 & 0 & 0 & 1 & 1 & 1 & 0 \\
12 & 0 & 0 & 1 & 0 & 0 & 0 & 0 & 0 & 0 & 0 & 1 & 1 & 0 \\
13 & 0 & 0 & 0 & 1 & 0 & 0 & 0 & 0 & 0 & 0 & 0 & 0 & 1 \\
\hline
\end{tabular}

Figura 6. Representação da matriz topológica para uma estrutura.

A primeira etapa da codificação é a numeração da substância, que respeita as seguintes regras :

1. Dois átomos ligados por uma ligação longa não podem apresentar números consecutivos. A ligação é dita longa quando ela é representada por um número ímpar de caracteres, maior que dois.

2. Os nós monoatômicos, ou seja, os átomos ligados por uma única ligação a um único átomo pertencente à cadeia, devem ser numerados em primeiro lugar.

3. A numeração da estrutura deve ser feita de modo que o programa possa reconhecer quais são as ligações alfa e beta. Portanto, foi estabelecido que se um átomo estiver em posicão alfa, todas as suas ligações com átomos de maior numeração serão consideradas alfa, o mesmo sendo aplicado às ligações beta. 
A próxima etapa é a elaboração do vetor nó, que deve conter a seguinte sequência de informação:

1. Os números iniciais e finais de cada cadeia.

2. Precedido por -1 , o número dos átomos aos quais os nós monoatômicos estão ligados, seguidos pelos números dos átomos não incluídos nas cadeias.

3. Precedido por -2, os números atômicos dos heteroátomos e suas posições.

4. Precedidas por -3 , as posições dos átomos das ligações duplas.

5. Precedida por -4 , a posição de um átomo do ciclo aromático que faça parte de apenas um anel.

6. Precedidas por -5 , as posições dos átomos das ligações triplas.

7. Precedidos por $-6-1$, os átomos beta, por -2 , os átomos alfa, por -3 , os átomos com carga positiva e por -4 , aqueles com carga negativa.

8. Precedidos por -7 , os átomos das ligações transanulares de conformação cis.

9. Precedidos por -8, os códigos e as posições dos macronós.

10. O sinal -9 , para fechar este primeiro vetor.

O sesquiterpenó da figura 7 será, portanto representado pelo seguinte vetor nó:

0717-113101717091707160813-2080203-30509-6-10116-20203-9.

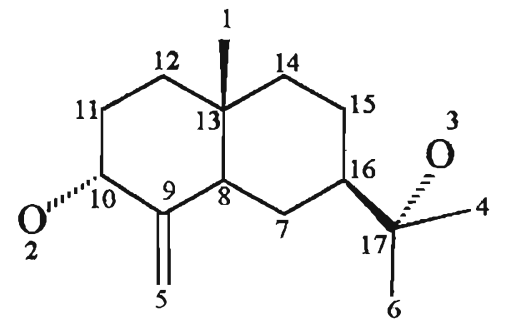

Figura 7. Exemplo utilizado para obtenção dos vetores. 
O vetor nó permite ao programa reconhecer o tipo de ligação, os tipos de átomos e as cadeias existentes na molécula, mas faltam ainda outras informações para que o computador possa transformar a matriz topológica numa representação gráfica. Estas informações são as indicações das direções das ligações que estão armazenadas no vetor passo. Este contém as coordenadas do átomo inicial da cadeia e as orientações sucessivas das ligações que a compõem, codificadas de acordo com a figura 8. O vetor final será o resultado da concatenação dos vetores nó e passo.

Para o exemplo da figura 7, o vetor passo será : 182301070102030503050605.

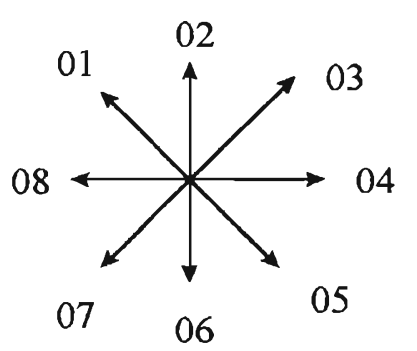

Figura 8. Codificação para as orientações das ligações.

Como a numeração dos átomos para a codificação da molécula não tem qualquer relação com a numeração biogenética, utilizada na química de produtos naturais, criou-se um vetor, chamado vetor biogenético, que correlaciona as duas numerações. O primeiro elemento do vetor biogenético é o número dado ao átomo que corresponde à posição biogenética 1 e assim sucessivamente.

Para identificação de cada estrutura pelo computador, foi criado um número denominado identificador, que é formado pelos cinco primeiros algarismos da maior raiz do determinante secular da matriz topológica. Isto permite uma fácil localização de estruturas dentro do banco de dados. 


\section{METODOLOGIA}

\section{1- O Levantamento Bibliográfico}

Para a construção do banco de dados foi realizado um levantamento bibliográfico dos dados espectrais de RMN ${ }^{13} \mathrm{C}$ de sesquiterpenos, utilizando-se os periódicos Phytochemistry (1970-1997) e Journal of Organic Chemistry (1975-1995), o review "Carbon-13 NMR of Natural Products. Monoterpenes and Sesquiterpenes. Attaur-Rahman, Ahmad, Plenum Publishing Co. Ltd., New York, vol. 1 (1992)" e as referências disponiveis no Instituto de Química da USP, citadas no Chemical Abstract (1967-1997), cujos números de registro foram obtidos através de pesquisa via on line, com as palavras-chave "sesquiterpen*" e "NMR".

\section{2- A Construção do Banco de Dados}

Os dados obtidos a partir do levantamento bibliográfico foram transferidos para formulários apropriados, que consistem de um espaço para o desenho da estrutura da substância e uma área onde são anotados os seguintes dados: número da ficha, nome trivial, classe química, tipo de esqueleto, dados botânicos (família, gênero e espécie), referência bibliográfica e dados físico-químicos. Para este trabalho foram pesquisados apenas os sequiterpenos que apresentavam dados de $R M N{ }^{13} \mathrm{C}$ atribuídos. Consequentemente, os dados botânicos presentes no banco de dados são escassos, comparados com o grande número de ocorrências botânicas de sesquiterpenos presentes na literatura.

Para a obtenção de deslocamentos químicos característicos de esqueletos ou subesqueletos, foi padronizada uma numeração para cada tipo de esqueleto, que nem sempre é idêntica à numeração biogenética. Isto deve-se ao fato de serem encontradas na literatura diferentes numerações para um mesmo tipo de esqueleto, sendo utilizadas aqui aquelas mais comumente encontradas.

Os solventes utilizados na obtenção dos espectros também foram considerados no armazenamento de dados, sendo que a grande maioria dos sesquiterpenos foram analisados em $\mathrm{CDCl}_{3}$. Por isso, durante as análises agrupamos todos os dados.

Pode-se observar, pelo levantamento realizado, a grande diversificação estrutural dos sesquiterpenos, o que torna esta classe de compostos um grande desafio para os espectroscopistas. A base de dados do SISTEMAT contém 2306 espectros de sesquiterpenos, distribuídos em 288 esqueletos (Figura 9). 
Figura 9. Esqueletos de sesquiterpenos presentes no banco de dados.<smiles>CC(C)=C1CCC2(S)C([13CH3])CCC[C@H](C)[C@H]2C1</smiles>

1. Eudesmano<smiles>CC(C)C1CCC2C(C)CCC(C)C2C1</smiles>

4. $15(\rightarrow 1)$-Eudesmano<smiles>CC(C)C1CCC2CCCC(C)C2C1</smiles>

7. 14-Nor-eremofilano<smiles>CC(C)C1CCC([AlH2])C2CC[C@@H](S)CC12</smiles>

10. Cadinano<smiles>[3H][C@@]1([Hg])CCC[C@]2(C)C(C)C(I)CCC12</smiles>

13. Drimano<smiles>CC(C)C1CCC2CCCC(C)C2C1</smiles>

2. 15-Nor-eudesmano<smiles>CC1CCCC2CCCCC12</smiles>

5. 11, 12, 13-Trinor-eudesmano<smiles>CCCCC1[C]CCCC1</smiles>

8. 11, 12, 13-Trinor-eremofilano<smiles>CC1CCC2CCCC(C(C)C)C2C1</smiles>

11. 14-Nor-cadinano<smiles>CC1CCC2(C)C(C)C(C)CCC2C1C</smiles>

14. $14(\rightarrow 3)$-Drimano<smiles>CC(C)C1CCC2(C)CCCCC2C1</smiles>

3. 14-Nor-eudesmano<smiles>CC(C)C1CCC2CCCC(S)C2([Hg])C1</smiles>

6. Eremofilano<smiles>CCC1CCC2CCCC(C)C2(C)C1</smiles>

9. 13-Nor-eremofilano<smiles>CC12CCCCC1CCCC2</smiles>

12. $11,12,13,15$-Tetranor-eremofilan<smiles>[Y3]C1([As])CCCC2([Y])CCCCC12</smiles>

15. 11-Nor-drimano 
<smiles>CC1CCC2C(C)C(C)CCC2(C)C1</smiles>

16. 11-Nor-14 $(\rightarrow 3)$-drimano<smiles>CC1CCC2C(C)CCCC2C1C</smiles>

19. 13, 14-Dinor-drimano<smiles>CC(C)=C1CCCC2CCCC(S)C12C</smiles>

22. Nardosinano

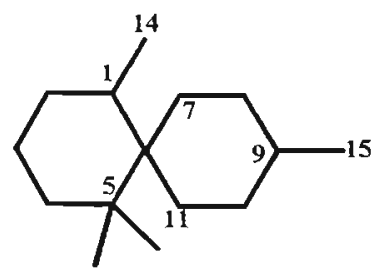

25. Chamigrano

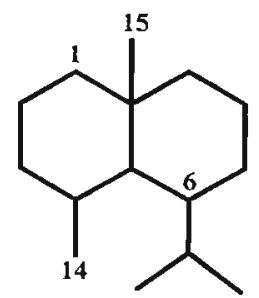

28. Gorgonano

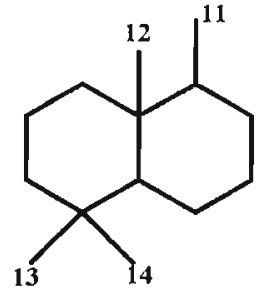

17. 12-Nor-drimano<smiles>[3H]C12CCCC(C)(C)C1CC(I)CC2</smiles>

20. 11-Nor-12(->7-drimano<smiles>CCC1CCCC2CCCC([AlH2])C12C</smiles>

23. 13-Nor-nardosinano

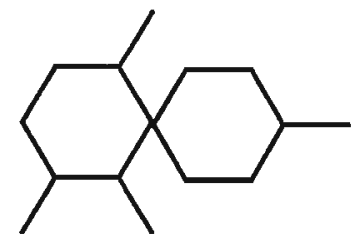

26. 12(->4)-Chamigrano<smiles>C=C1C([Hg])CCC2CC(C(C)C)CCC12</smiles>

29. Esq 18<smiles>CC1CCC2C(CCCC2(C)C)C1(C)C</smiles>

18. $13(->9)-$ Drimano<smiles>CC(C)C1CCC2CCCC(C)C2C1C</smiles>

21. 14(->6)-Eremofilano<smiles>CC(C)=C1CC[C@]2(C)C(C)CCC(C)C2C1</smiles>

24. $\operatorname{Esq} 7$

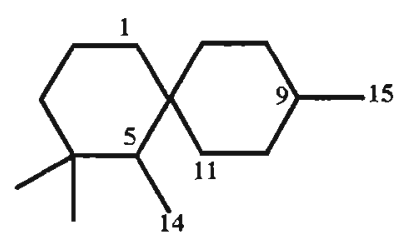

27. Isochamigrano

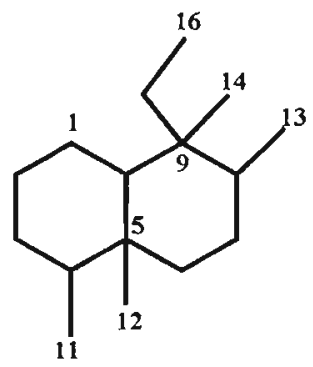

30. Esq 38 


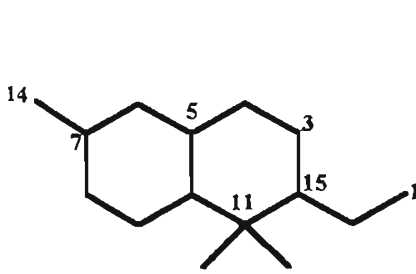

31. Furodisano<smiles>CC1CCC2CCC3C(C3(C)C)[C@@]2(C)C1C</smiles>

35. Aristolano

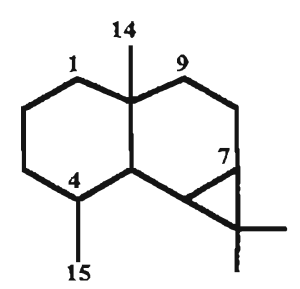

36. Maliano

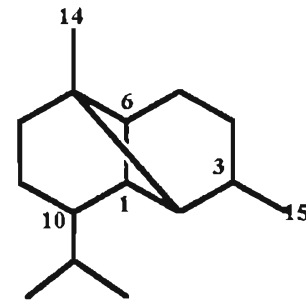

33. Copaano<smiles>CC1CCC(C)C2C1CCC1C2C1(C)C</smiles>

37. $14(\rightarrow 1)-M a l i a n o$

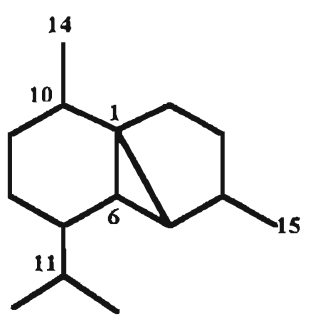

34. Epicubebol

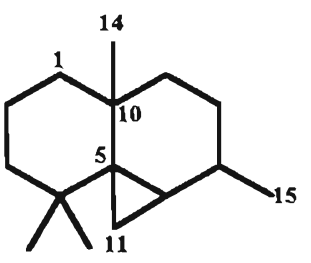

38. Tujopsano<smiles>CC(C)C1CCC2(S)C3CC(CC3C)C2(C)C1</smiles>

39. 1-3-Eudesmano<smiles>CCC1C([12CH2])C[C@@H]2CC(C)(C)CC2C1[13CH]</smiles>

43. Pterosin

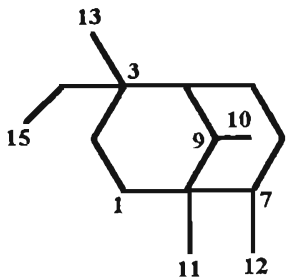

40. Trifarano<smiles>CCC1C(C)CC2CC(C)CC2C1C</smiles>

44. 11-Nor-pterosin

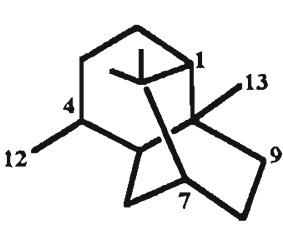

41. Pre-seichelano

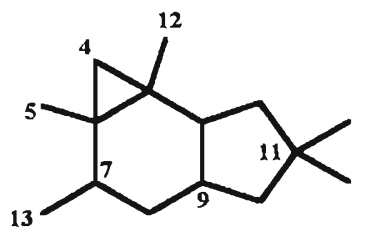

42. Marasmano

45. Aliacano

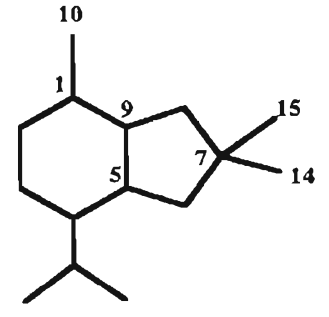

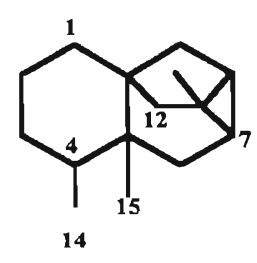

46. Ishwarano

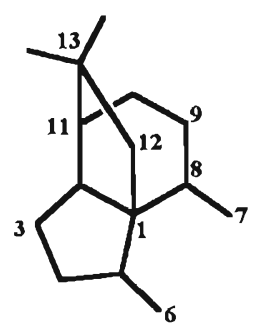

47. Esq 32<smiles>CC1(C)CC2CC2([13CH3])C2CC(S)CCC21</smiles>

48. Lipifoliano

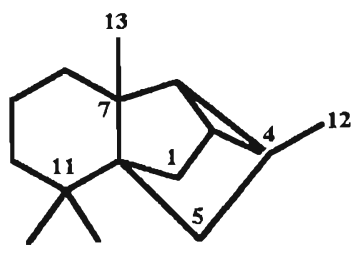

49. Ciclomiltailano

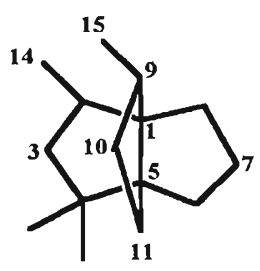

50. 15(->9)-Modefano 


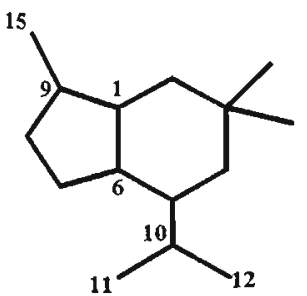

51. Brasilano<smiles>CC1(C)CC([AlH2])([AsH3])C2C(=O)C([AlH2])CCC21</smiles>

55. Botriano

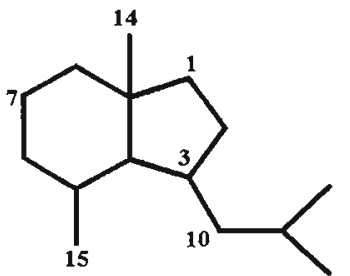

59. Bulatano<smiles>CC1CCC2([18F])CC1(C)CCC2C</smiles>

52. Pinguisano

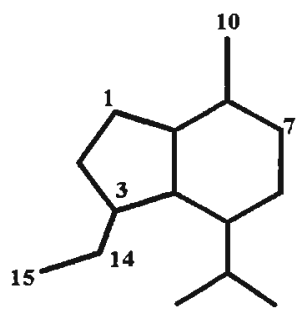

56. Oplopanano

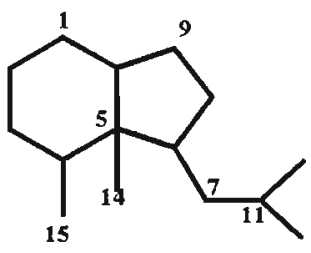

60. Chiloscifano<smiles>CC(C)C1CCC2(C)C([AlH2])C([Hg])CCC12</smiles>

63. Esq 31<smiles>CC(C)C[C@H]1C(C)CCC2(C)C([As])CCC12</smiles>

64. Hidrindano<smiles>CCC1CCC2(C)CCCC2(C)C1</smiles>

53. 15-Nor-pinguisano<smiles>CC(C)C1CCCC2CCC([13CH3])C21</smiles>

57. 10-Nor-oplopanano<smiles>CC(C)=CC1CCC([AlH2])[C@@H]2CCC(C)[C@H]12</smiles>

62. $13(->10)-V a l e r e n a n o$

54. 10, 11,15-Trinor-pinguisano

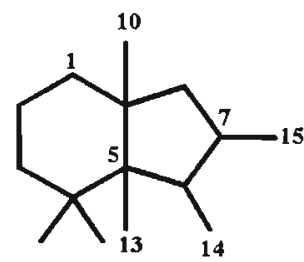

58. Thapsano

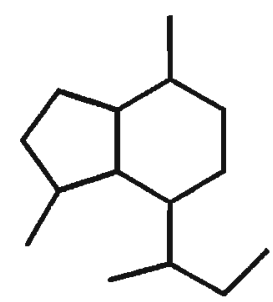

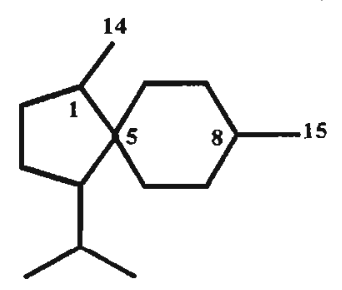

65. Acorano
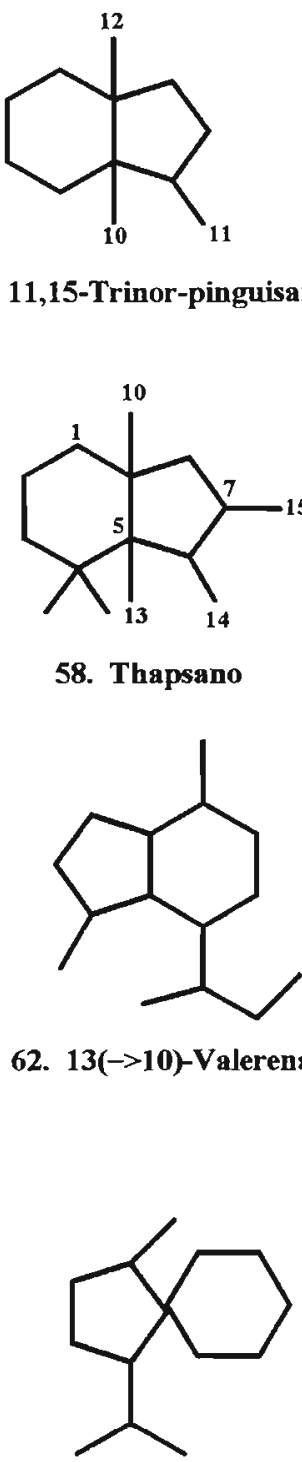

66. 15-Nor-acorano

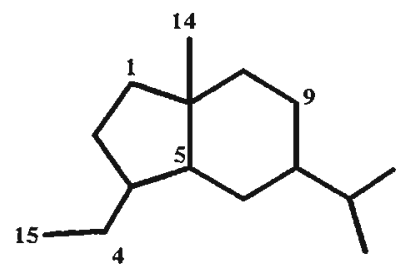

69. Ipionano

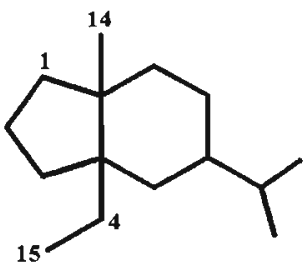

70. Isoipionano
68. Esq 12

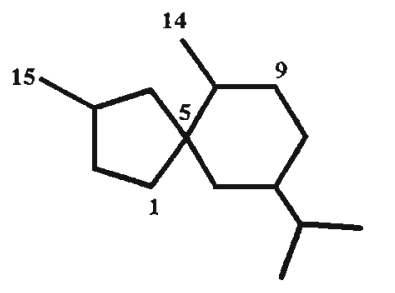

67. Esq 11<smiles>CC(C)C1CC[C@@H]([AlH2])[C@]2(CCC([Hg])C2)C1</smiles> 
<smiles>CC1CCC(C2(I)CCC(C)C2[18F])CC1</smiles>

71. Laurano

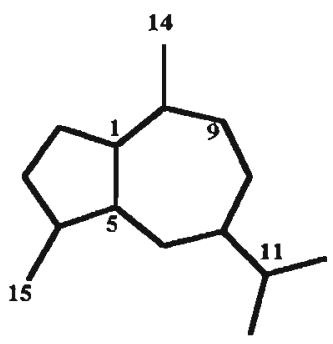

75. Guaiano

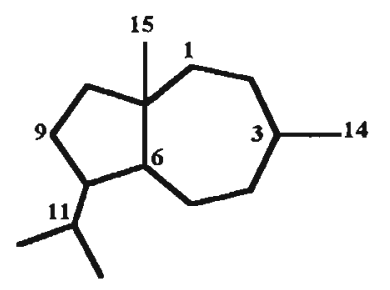

79. Carotano

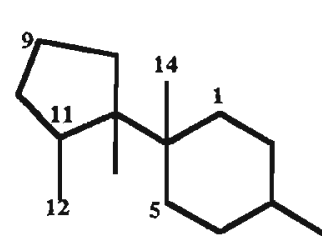

72. Tricotecano

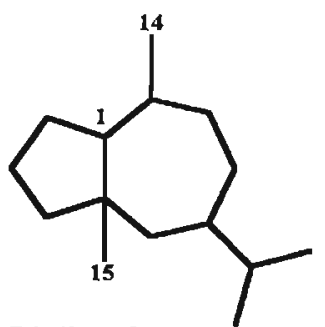

76. Pseudo-guaiano

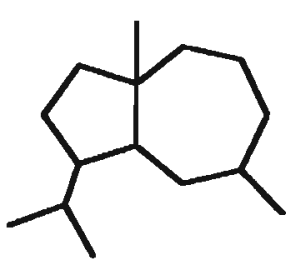

80. $14(->4)-$ Carotano

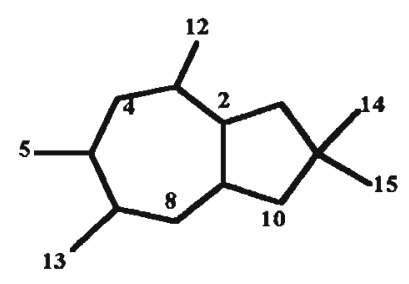

83. Lactarano

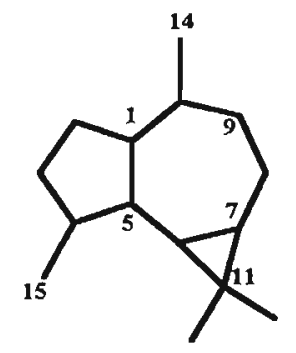

87. Aromadendrano

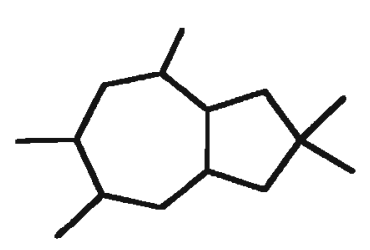

84. $5(->4)$-Lactarano

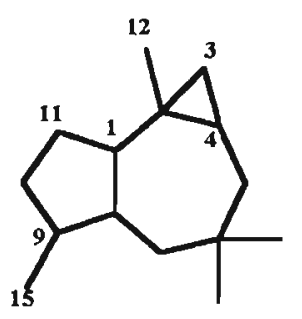

88. Africano

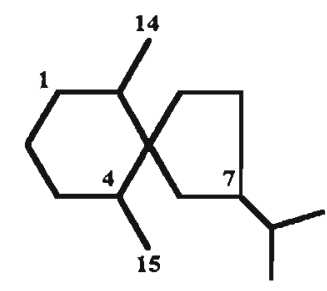

73. Vetispirano

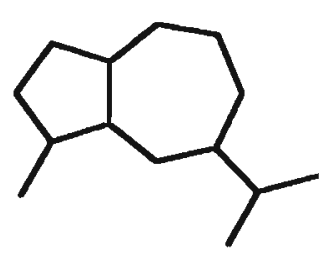

77. 14-Nor-guaiano

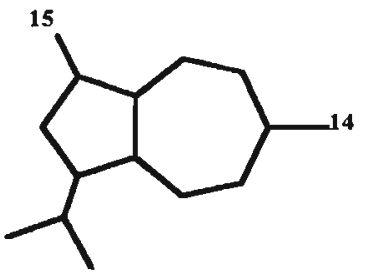

81. Isocarotano

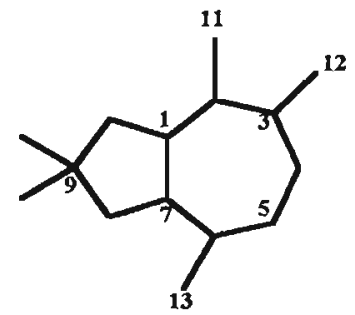

85. Tremulano

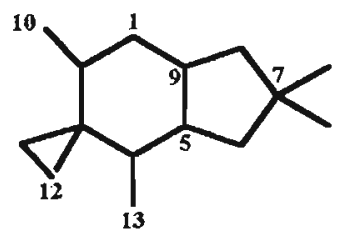

74. Iludano

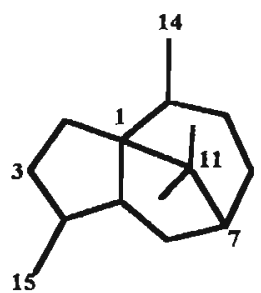

78. Ciperano

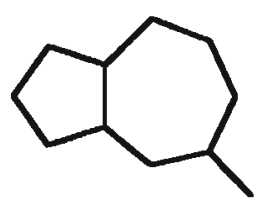

82. Tetranor-carotano

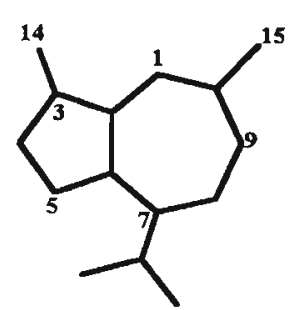

86. Esq 25

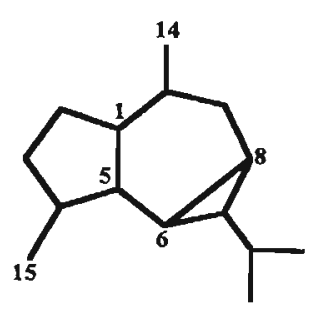

89. 6-8-Guaiano

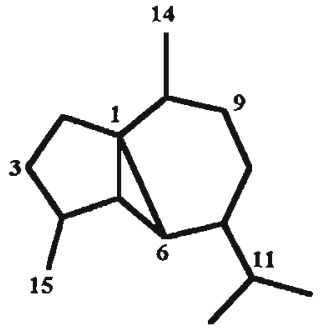

90. Cubebano 


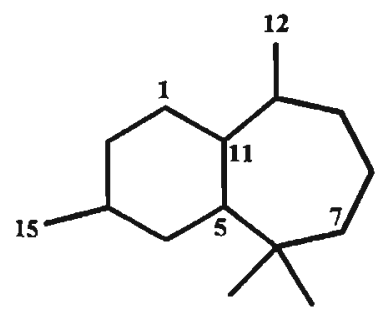

91. Himachalano

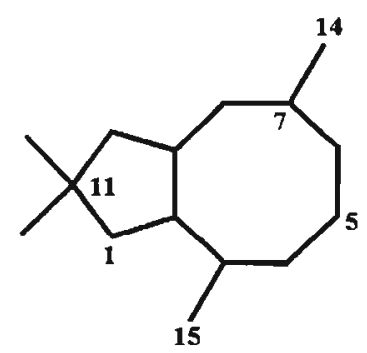

94. Asteriscano<smiles>CC(C)C1C(C)C([Hg])CCC2C1C2(C)C</smiles>

97. 2,3-Seco-aromadendrano

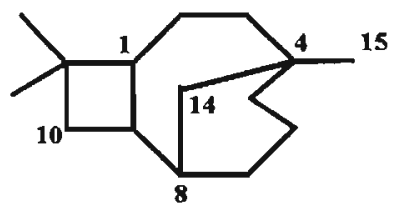

100. 4-14-Cariofilano

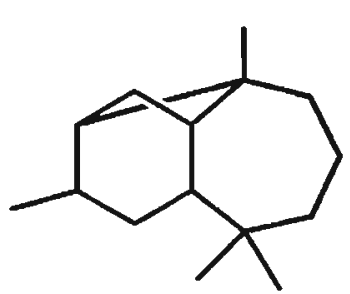

92. 2-10-Himachalano

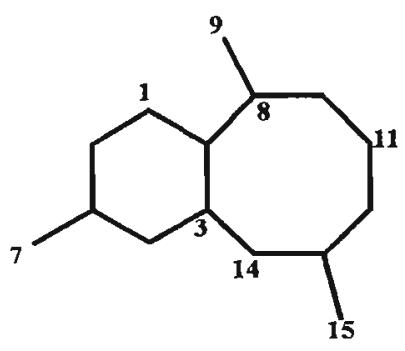

95. Esq 19

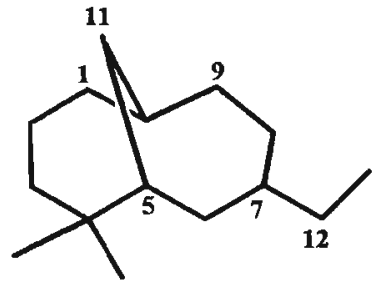

98. Espiniferin

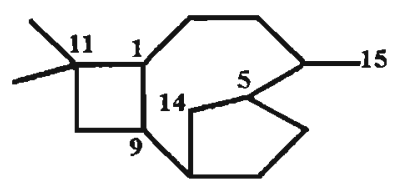

101. 5-14-Cariofilano

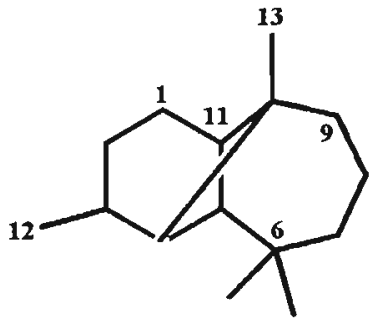

93. Longipinano<smiles>[Y]C1CCCCC2CCCC([As])C2(C)C1</smiles>

96. Neolemnano

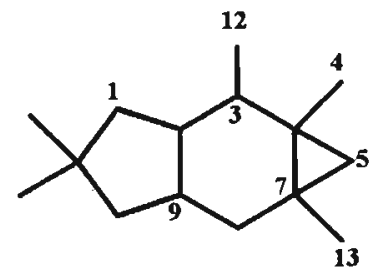

99. Isolactarano

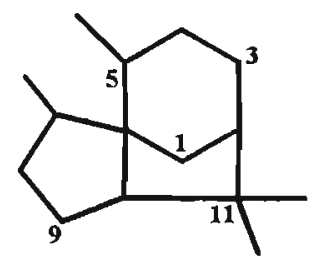

102. Isocedrano

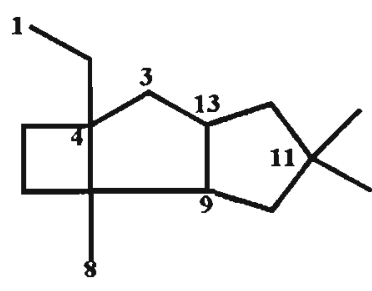

103. Esq 30

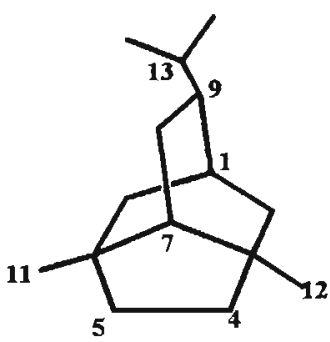

104. Esq 34 


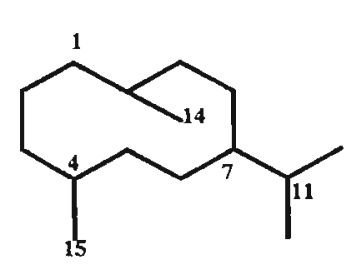

105. Germacrano

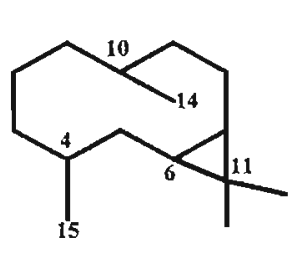

106. Biciclogermacrano<smiles>CC1CCCC2C(C1)C2(C)C</smiles>

107. Lepidozano

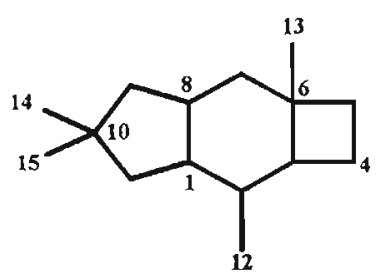

108. Esterpurano

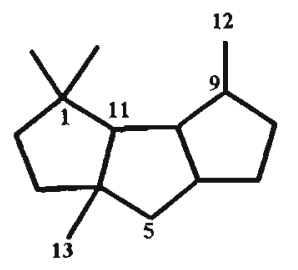

109. Capnelano

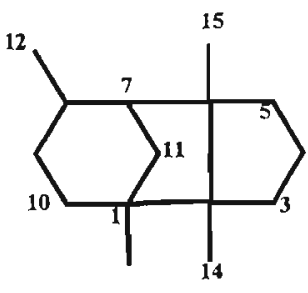

113. Gimnomitrano

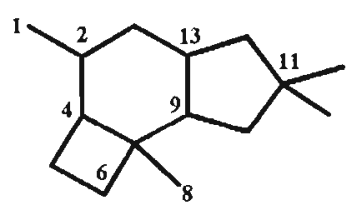

117. Protoiludano

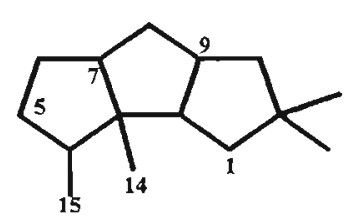

110. Hirsutano

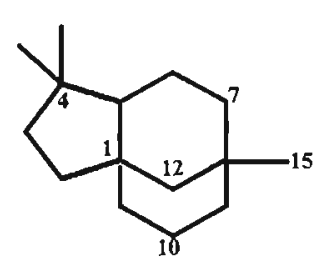

114. Clovano

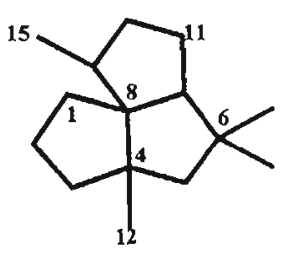

118. Silfinano

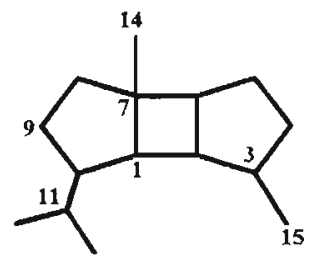

111. Bourbonano

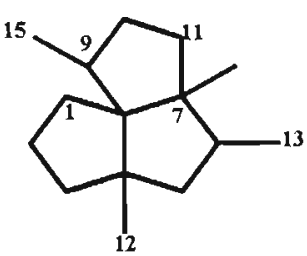

115. Isocomano

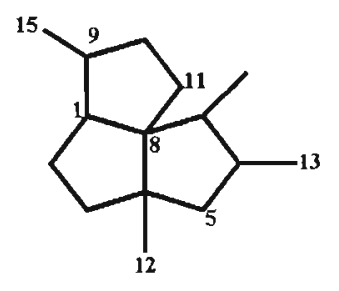

119. Silfiperfolano<smiles>[Y]C1CCCC(C)(C)CCC([125I])CC1</smiles>

123. Humulano

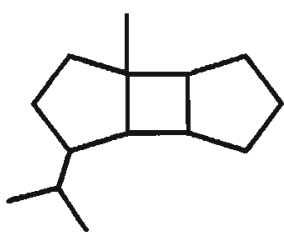

112. 15-Nor-bourbonano

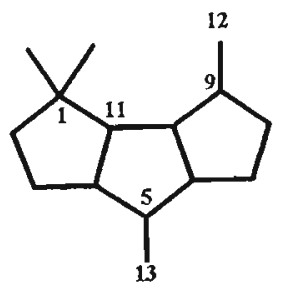

116. Isocapnelano

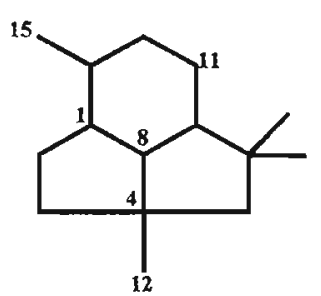

120. Presilfiperfolano

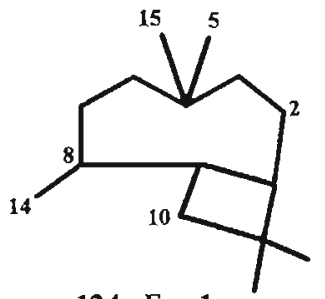

124. Esq 1

121. Cedrano

122. Cariofilano

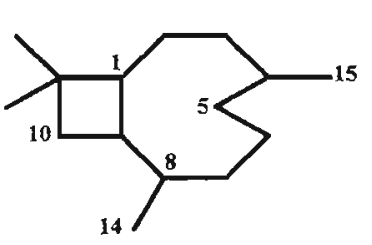




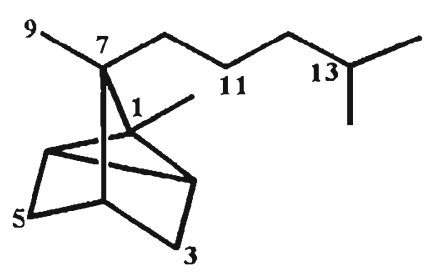

125. o-Santalano<smiles>[Y]C(C)C1CC(C(C)C)CCC1([AsH3])[Hg]</smiles>

128. Elemano

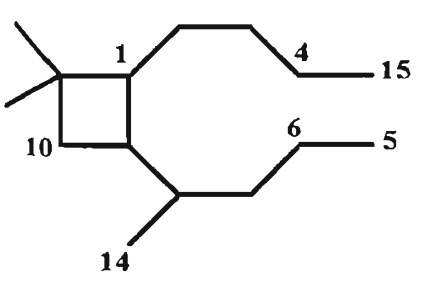

131. 4,5-Seco-cariofilano

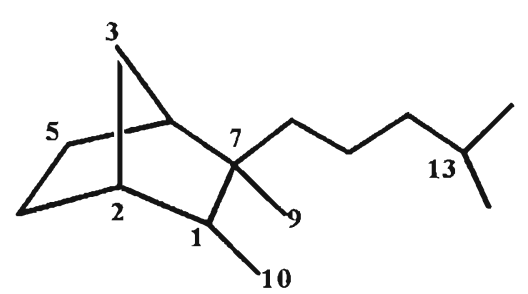

126. $\beta$-Santalano<smiles>CC(C)=[13CH]CCC([AlH2])[C@H]1[CH]CC([AsH])CC1</smiles>

129. Bis abolano

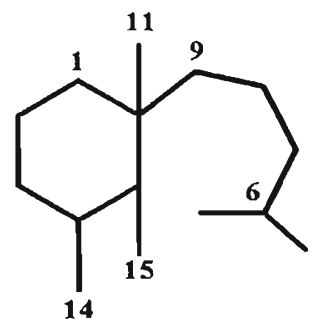

132. Oxepine<smiles>C=C(CC)C1CCCCC1CCC</smiles>

135. Esq 24

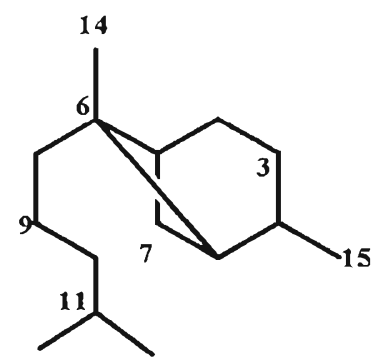

127. Bergamotano<smiles>CCCC1[C@@H](C(C)C)CCC(I)C1(C)C</smiles>

130. $3,4-\operatorname{Seco}-13(->9)$-drimano

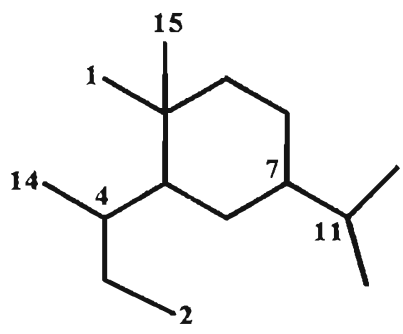

133. Fituberin<smiles>CC(C)CCCC([Al])CCCC([As])CI</smiles>

134. Farnesano

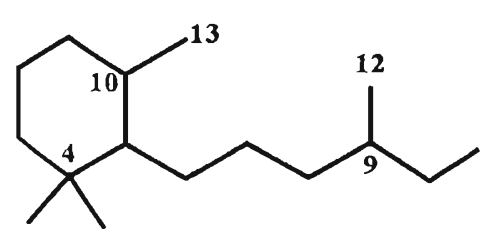

137. 9,10-Seco-drimano<smiles>CC1(C)CCCC([AlH2])C1CCC([AsH3])C[TlH]</smiles>

138. Monociclofarnes ano<smiles>CCCCC1C(C)CCC(C)C1C</smiles>

136. Esq14<smiles>CCC(S)CCC1C(C)CCC([Hg])C1[Tl]</smiles>

139. 13( $(\rightarrow 2)$ - Monociclofarnes ano 
<smiles>CC(C)CCC1C(C)CCC(C(C)C)C1c1ccc2c(c1)CCPC2</smiles>

140. Esq 60

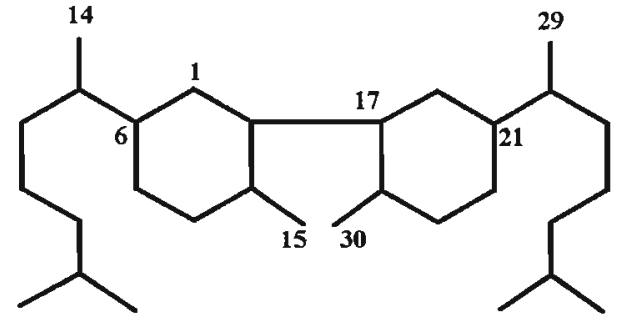

141. 2, 2'-Bis-bisabolano<smiles>CC1CCCC([AlH2])(CCC2(S)CCc3ccccc3O2)C1C</smiles><smiles>CC1CCC(I)C(C)(CCC(S)CCC2CCCCC2)C1C</smiles>

143. Esq 27<smiles>CC(C)=CCC[C]([AlH])CCCC([135I])CCC1COc2ccccc2C1</smiles>

144. Esq 37

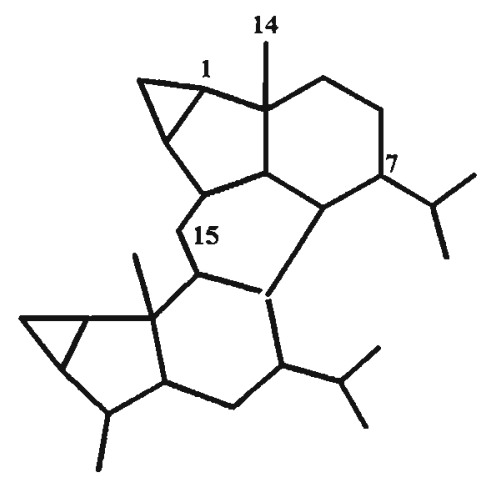

145. $6-8$ ', 15-9'-Bis-lindenano 


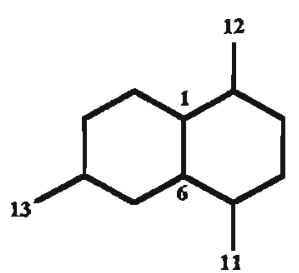

146. 12, 13-Dinor-cadinano<smiles>[Y]C1CCC(C(C)C)C2CCCC[C@@H]12</smiles>

147. 15-Nor-cadinano<smiles>CCC(S)CC1CC(C)CCC1C(C)C</smiles>

148. 1,2-Seco-cadinano<smiles>CCC(C)CC1CCCCC1C(C)C</smiles>

149. 14-Nor-1, 2-seco-cadinano<smiles>CC(C)=C1CC[C@](C)(C(C)CCI)C1</smiles>

150. 1, 10-Seco-eudesmano<smiles>CCCC(C)C1CCC(C(C)C)CC1C</smiles>

153. $15(->1)-4,5-$ Seco-eremofilano<smiles>CCC(CCC1CCCC([As])C1)C(C)C</smiles>

151. $14(\rightarrow \rightarrow)-5,6-$ Seco-eremofilano<smiles>CC1CCCC2CCC(C3CC3)CC12C</smiles>

154. 12-13-Eremofllano<smiles>CC(C)=C1CCC(C(C)CCCS)C(C)C1</smiles>

152. 4, 5-Seco-eremofilano

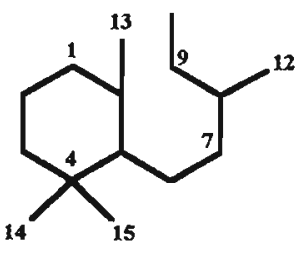

155. 9, 10-Seco-drimano<smiles>CCCCC1CCC(C(C)C)CCC1[As]</smiles>

156. 4, 5-Seco-guaiano

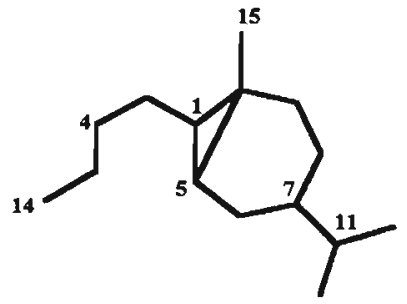

160. 5-10-4, 5-Seco-guaiano

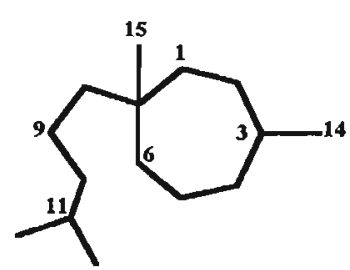

157. 6, 10-Seco-carotano

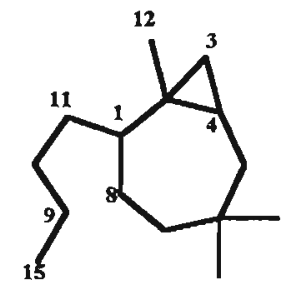

158. 8, 9-Seco-africano

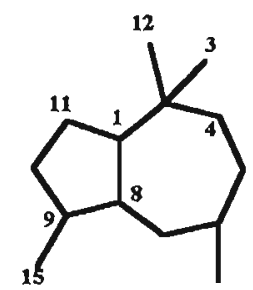

159. 3, 4-Seco-african

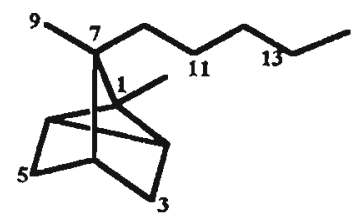

161. 15-Nor-a-Santalano

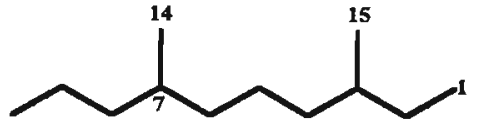

162. 11,12,13-Trinor-farnesano 


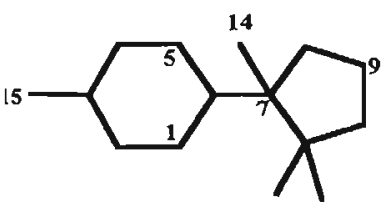

163. Cuparano
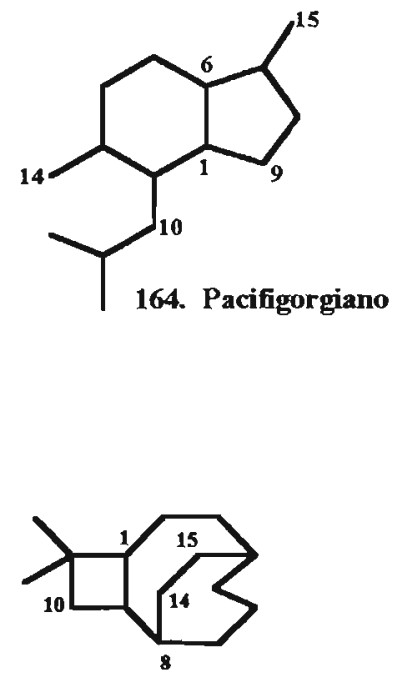

168. 14-15-Cariofilano

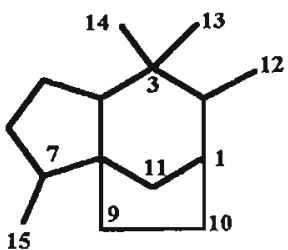

165. Prezizano

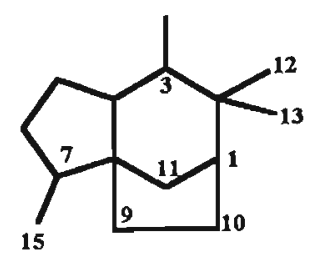

166. Zizano

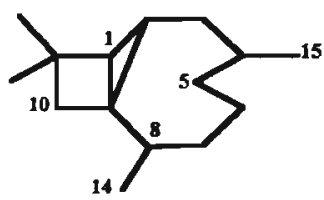

167. 2-9-Cariofilano

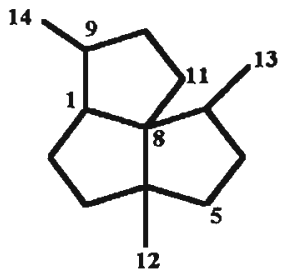

171. 13-Nor-silfiperfolano

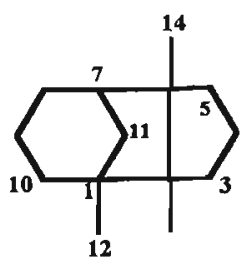

172. 12-Nor-gimnomitrano

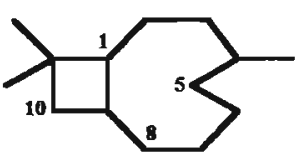

169. 14-Nor-cariofilano

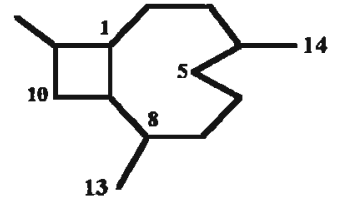

170. 12-Nor-cariofilano

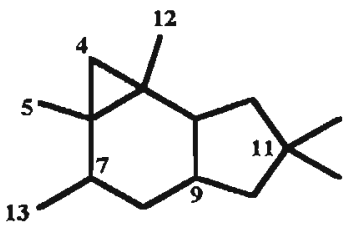

173. 13-Nor-marasmano

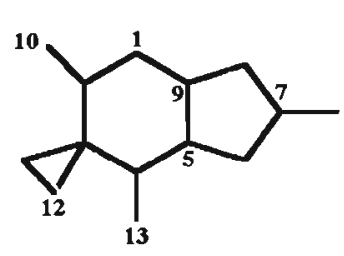

177. 15-Nor-iludano

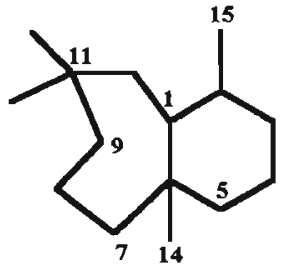

174. Esq 13

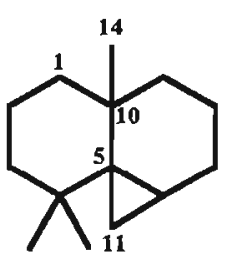

178. 15-Nor-Tujopsanc

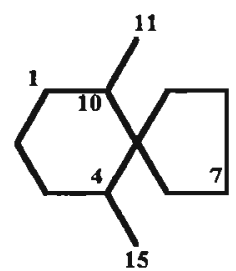

11,12,13-Trinor-vetispiran

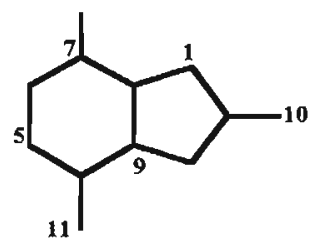

179. 11,13,14-Trinor-12(->4)-pterosin

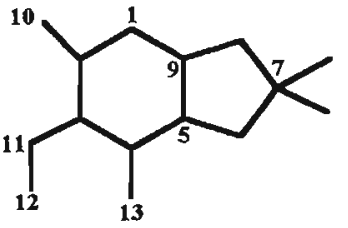

180. Iudalano

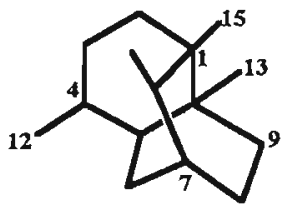

181. Seichelano

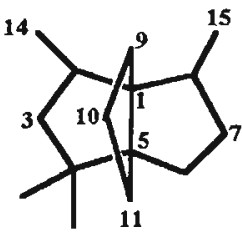

182. Modefano 


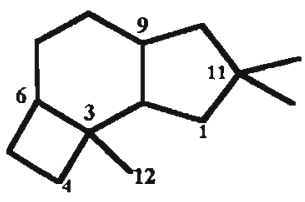

183. 1- Nor-protoiludano

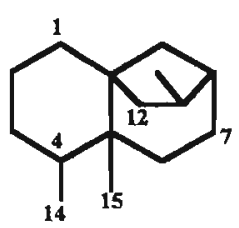

187. 7, 11-Seco-ishwarano

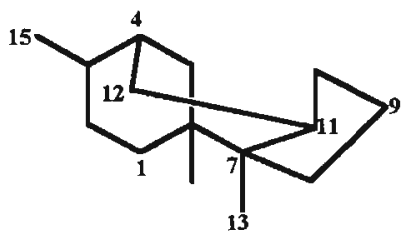

184. 4,12-Ciclo-tricotecano

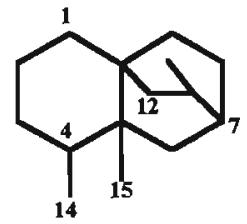

188. 8, 11-Seco-ishwarano

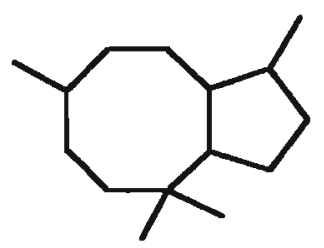

185. Ambiguano

186. $13,14,15$-Trinor-ishwarano
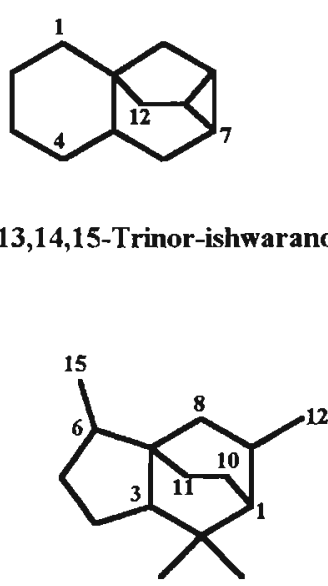

190. Esq 17

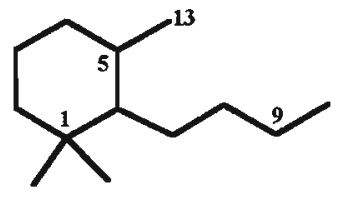

191. 11, 15-Dinor-monociclofarnesano

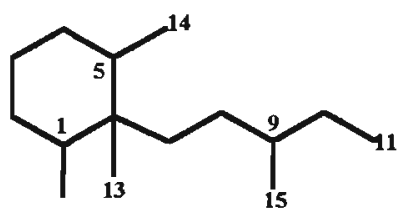

192. $13(->6)$-monociclofarnesano

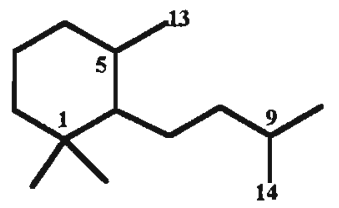

193. 11-Nor-monociclofarnesan!

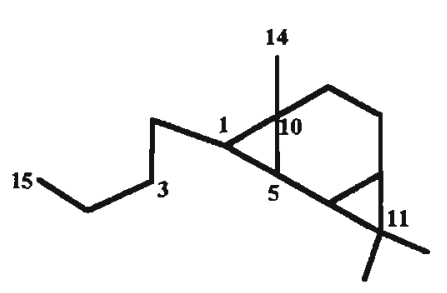

194. 5(4->10)-Abeoaromadendrano

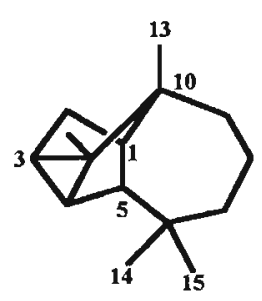

195. Longiciclano<smiles>CC(C)CCCC1(C)C2CCC(S)CC21</smiles>

196. Sesquicarano

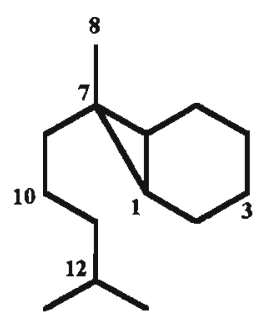

197. 15-Nor-sesquicaranc

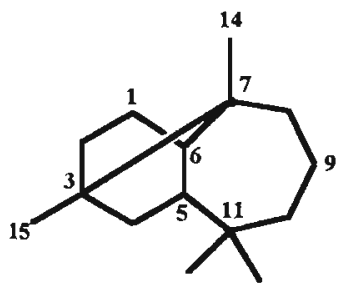

198. Longibornano

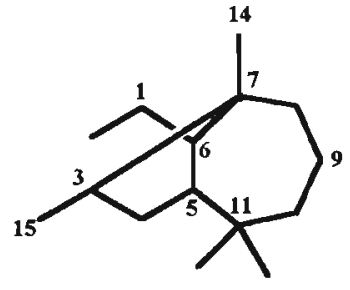

199. 2, 3-Seco-longibornano

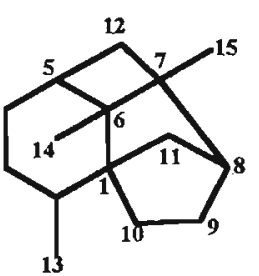

200. Solanascono

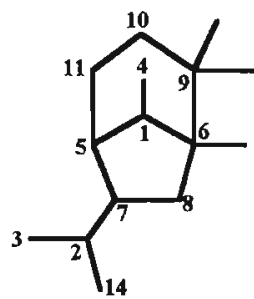

201. Esq 8 


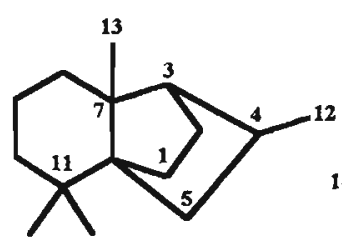

202. Miltailano<smiles>CCC1CCC2=C(CC1)C(S)C(C)CC2</smiles>

203. Esq 63<smiles>C/C=C\CCC(C)C1CCC(C)CC1</smiles>

204. 13-Nor-bisabolano

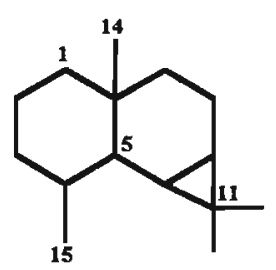

205. 14(->10)-Aristolano<smiles>CCCC(C)C1CCC(I)CC1</smiles>

206. 11, 12, 13-Trinor-bisabolano

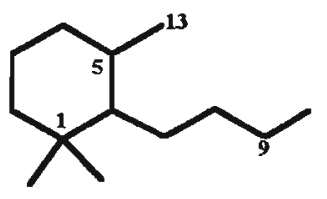

207. 10, 11-Dinor-monociclofarnesano

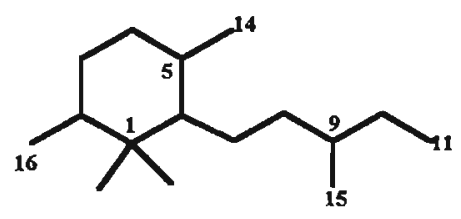

208. 2-Me-monociclofamesano<smiles>CC(C)CCCC(P)CC(C)C(C)I</smiles>

209. Esq 3

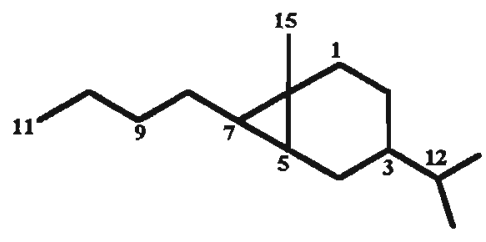

210. Esq 4<smiles>C=C(C)CCC(CC)C1CCCC([As])CC1</smiles>

211. Esq 6<smiles>CCCC[C@@H](CC1CCCC1C)C(C)C</smiles>

212. Esq 9<smiles>CC(C)C1CCCC2CCCC2(C)CC1</smiles>

213. Esq 10<smiles>CCC(CCC(C)C)C1CCC(S)CC1</smiles>

214. Esq 23<smiles>CC(C)C1CCC2C(C)CCCC(C)C2C1</smiles>

215. Esq 26

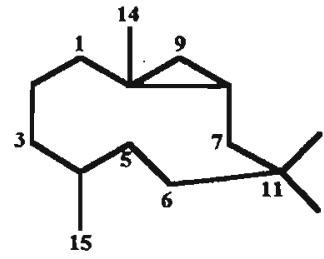

216. Biciclohumulano<smiles>CC(C)(CCCC[12F])[C@@H]1C[CH]CC([SiH3])C1</smiles>

217. 10, 11-Seco-himachalano<smiles>CCCCCC1(S)CCC(C(C)C)CC1</smiles>

219. 4, 5-Seco-Eudesmano

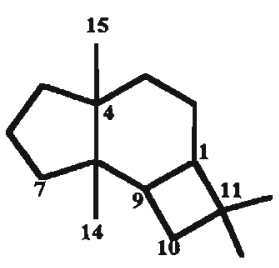

220. Punctatano 


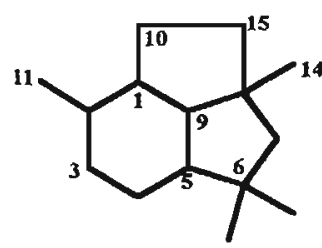

221. Probotriano

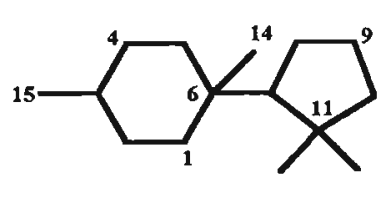

222. $14(->6)$-Cuparano

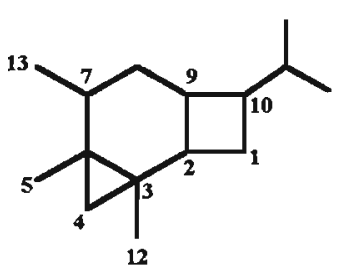

223. $1(11->10)$-Abeomarasmano

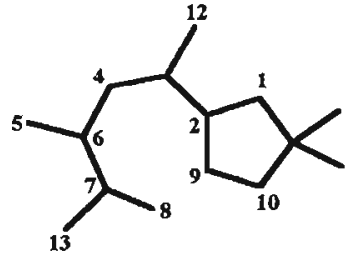

224. 8, 9-Seco-lactarano

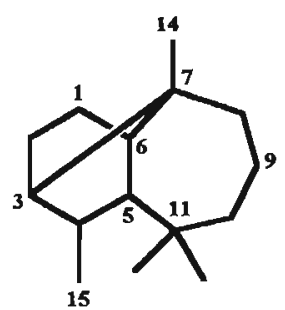

225. $15(->4)$-Longibormano

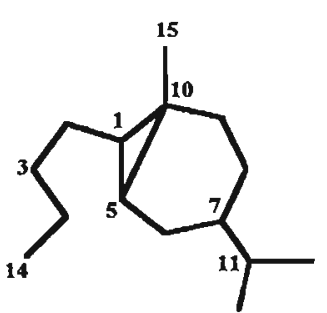

226. 5-10, 4, 5-Seco-guaiano

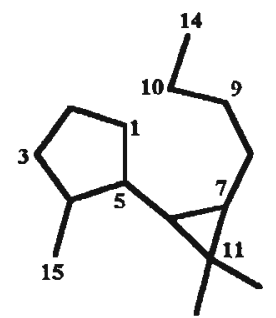

227. 1,10-Seco-aromadendrano

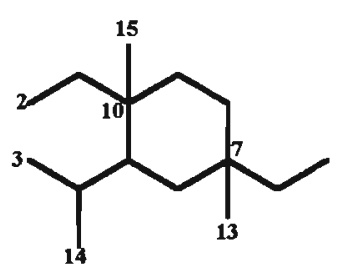

228. $13(->7)$-Elemano

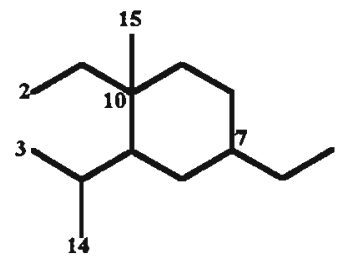

229. 13-Nor-elemano

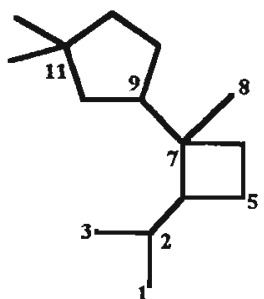

230. Fomanosin

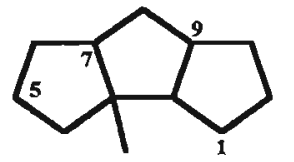

231. 12,13,15-Trinor-hirsutano

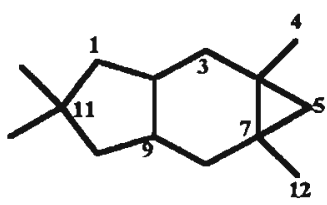

232. 12-Nor-isolactarano

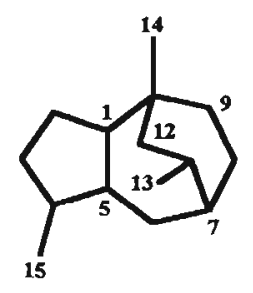

233. Rotundano

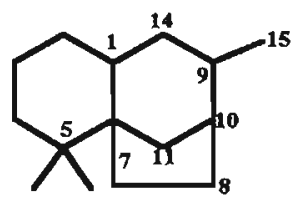

237. Omfalano

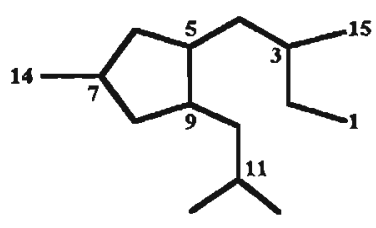

234. Esq 29

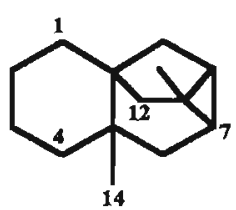

238. 14-Nor-ishwarano<smiles>CC(C)C1CCC(C)C(CCCS)C1C</smiles>

235. 4, 5-Seco-cadinano<smiles>CCC1CCC(C(C)C)CC1([Hg])C[13CH3]</smiles>

239. Esq 64<smiles>CCCCC(C(C)C)C1CCCC(C)C1</smiles>

236. 1, 10-Seco-cadinan<smiles>CCCC1CC(C(C)C)CCC1C</smiles>

240. Esq 57 


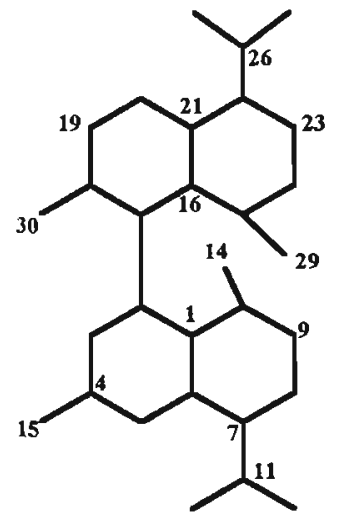

241. 2-Bis-cadinano

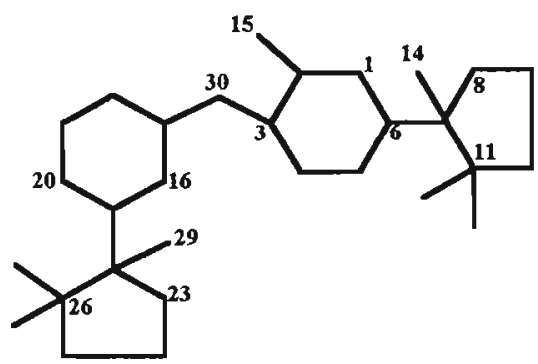

244. 3-15'-Bis-isocuparano

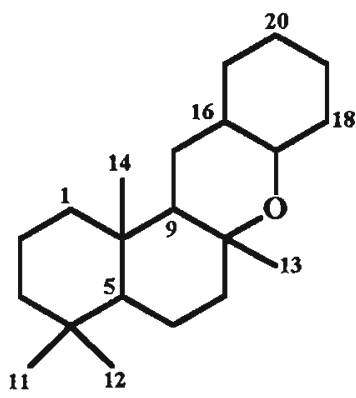

246. DRI-8O,15-CM

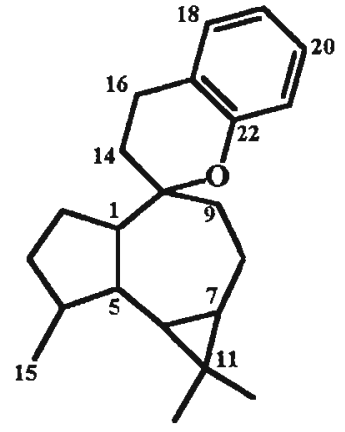

242. Aromad-14-CM1

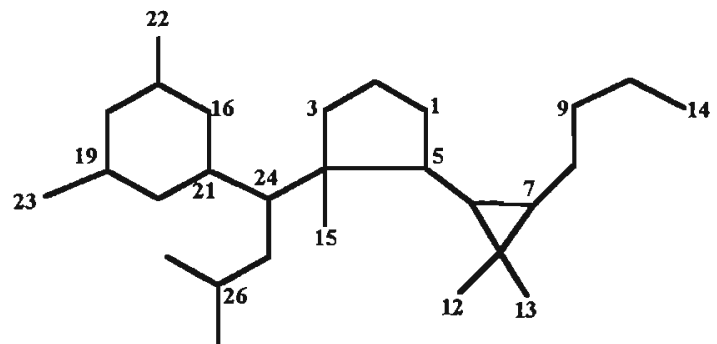

243. Esq 35<smiles>Cc1coc2c1C(Cc1oc3c(c1C)C([13CH3])[C@@H]1C(C)CCC(C)C31)C1CCCC(C)C21</smiles>

245. 12-14'-Bis-14(->6)-8,12-Furanoeremofilano

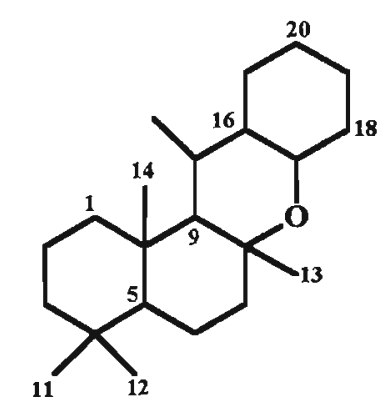

247. 15-Me-DRI-80,15-CM

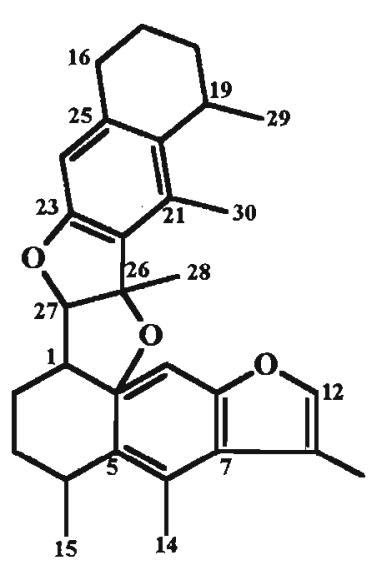

248. Esq 45

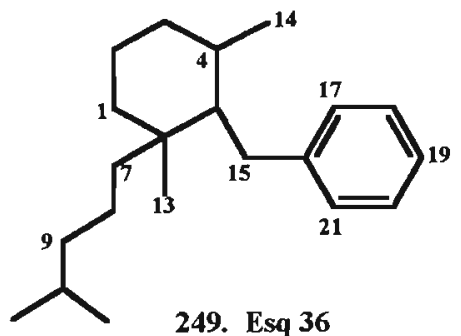

249. Esq 36

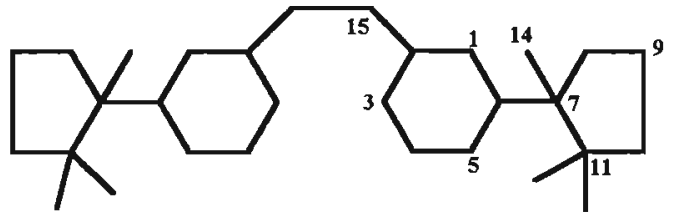

250. 15-15'-Bis-isocuparano 


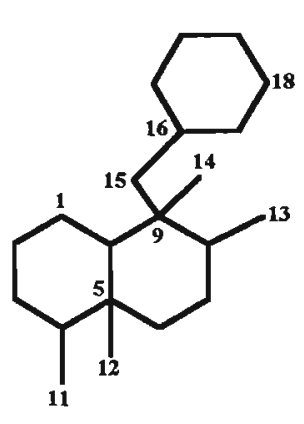

251. Esq 20

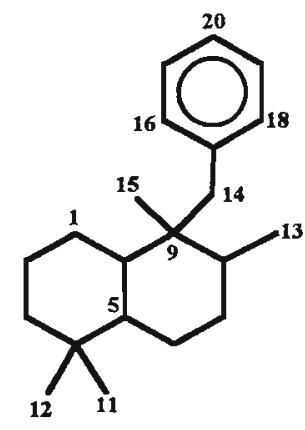

252. Esq 21

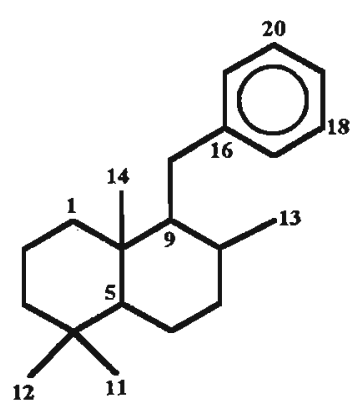

253. Esq 22

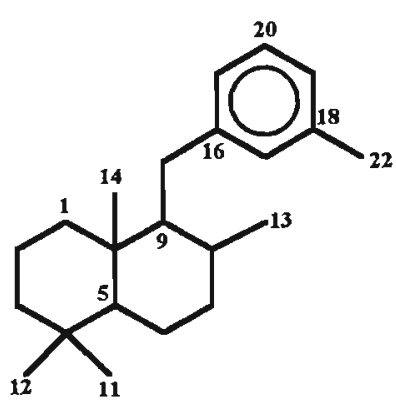

254. Esq 41<smiles>[Z]C(I)CCCC([Tl])CCC[C@@H](C)CCc1ccccc1C</smiles>

255. Esq 42

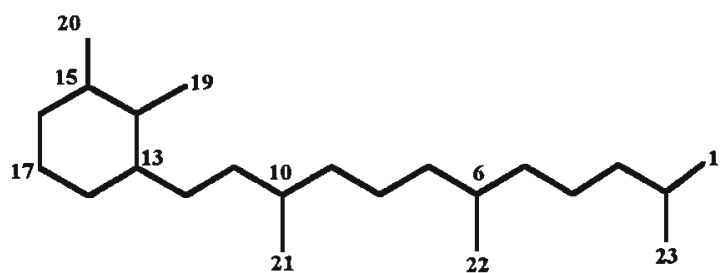

256. Esq 43

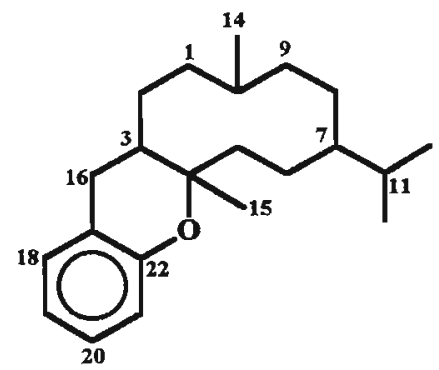

257. Esq 44

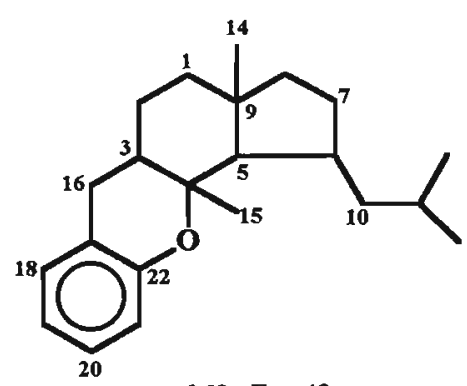

260. Esq 49<smiles></smiles>

258. EUD-3, 4O-CM

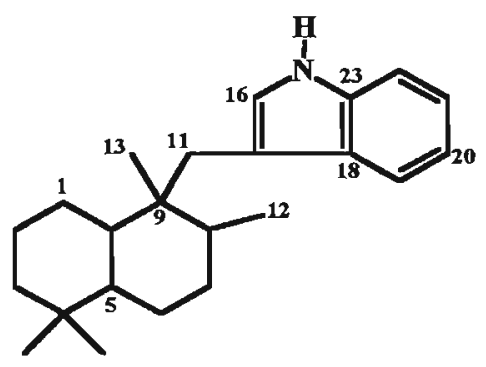

261. Esq 51 B

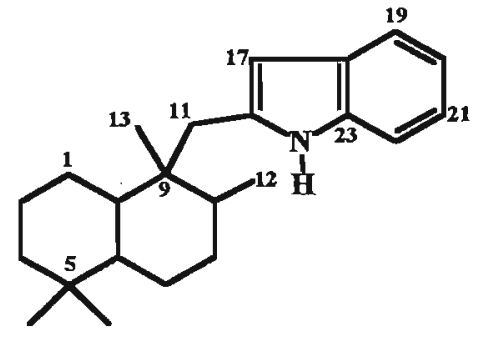

259. Esq 51 A

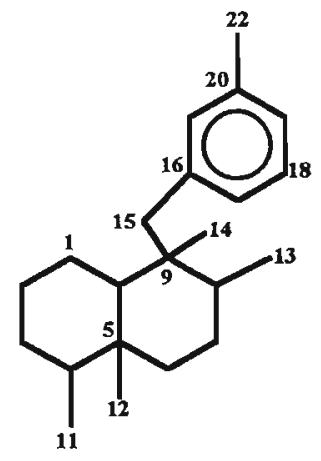

262. Esq 52 
<smiles>CC(C)C(C)CCCC1CCCCC1</smiles>

263. Esq 33

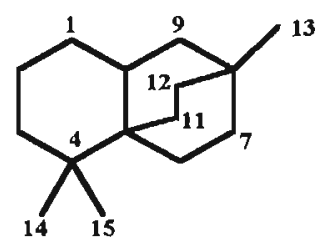

266. Esq 50

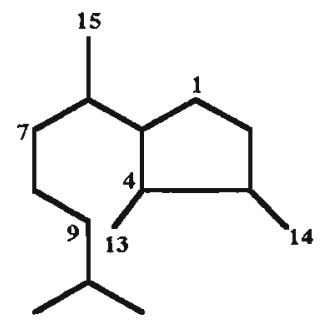

264. Esq 46

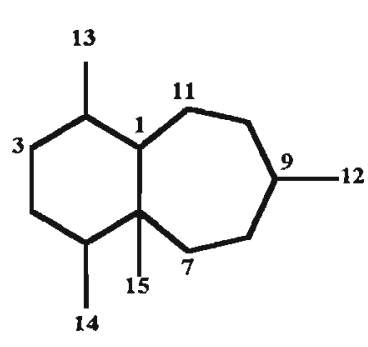

267. Esq 53

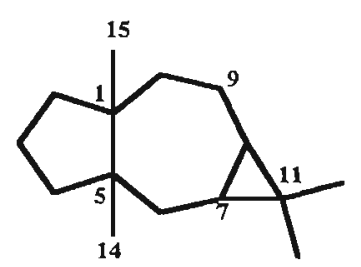

265. Esq 48

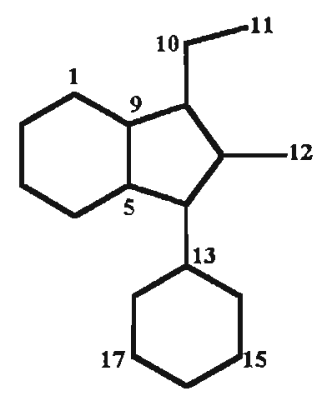

268. Esq 54

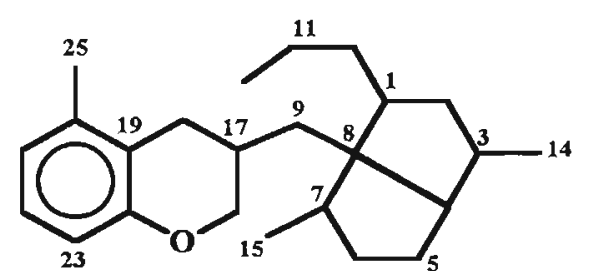

269. Esq 55

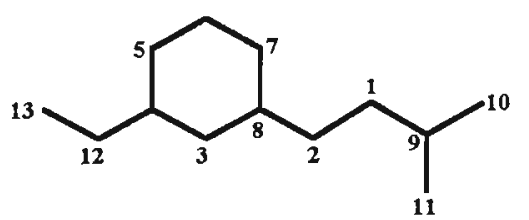

271. Esq 58

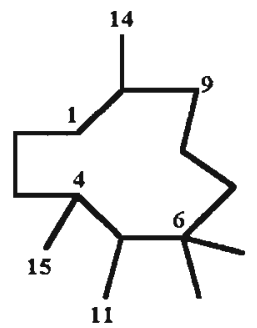

273. Esq 61<smiles>CC[C@H](C)CC[C@H](C)CCCI</smiles>

270. Esq 56

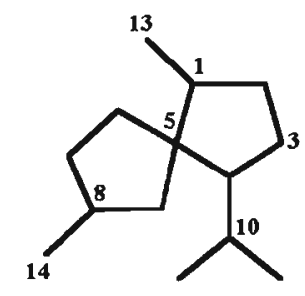

272. Esq 59

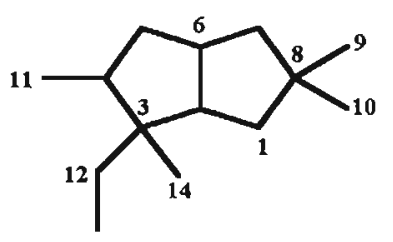

274. Esq 62 


\section{3- PROGRAMAS PARA CRIAÇÃO DE BANCOS DE DADOS}

O programa DATASIS permite a codificação automática dos vetores nó e passo $^{28}$, a partir do desenho da molécula na tela do computador. Estes desenhos podem ser armazenados em arquivos como esqueletos e/ou substâncias funcionalizadas para agilizar a entrada de novas substâncias no banco.

Ao iniciarmos o programa DATASIS, é mostrada uma tela solicitando o nome do arquivo de banco fonte, ou seja, o banco no qual são primeiramente armazenados os dados recolhidos da literatura, sendo depois solicitados os nomes dos arquivos de esqueletos e de substâncias. Logo em seguida, é mostrado o menu principal (Figura 10).

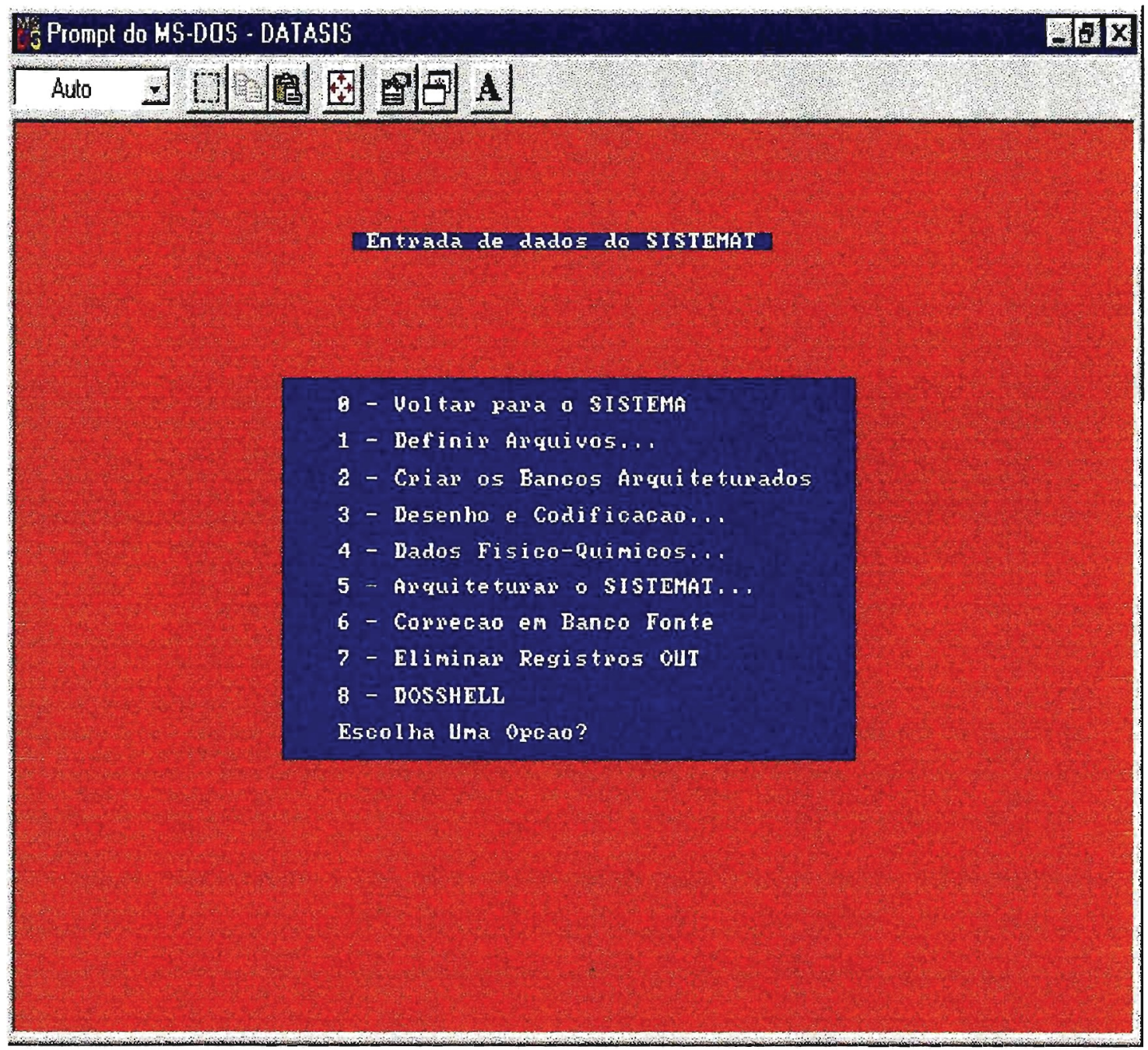

Figura 10. Tela principal do programa DATASIS. 
A opção 3 leva a outro menu que permite ao usuário voltar ao menu anterior, desenhar uma estrutura, verificar a possibilidade de codificação da mesma e, por último, iniciar a entrada dos dados da substância. Na figura 11 é mostrada a tela de desenho do programa DATASIS, que apresenta opções para manipulação dos arquivos e os símbolos usados para representação dos átomos e ligações.

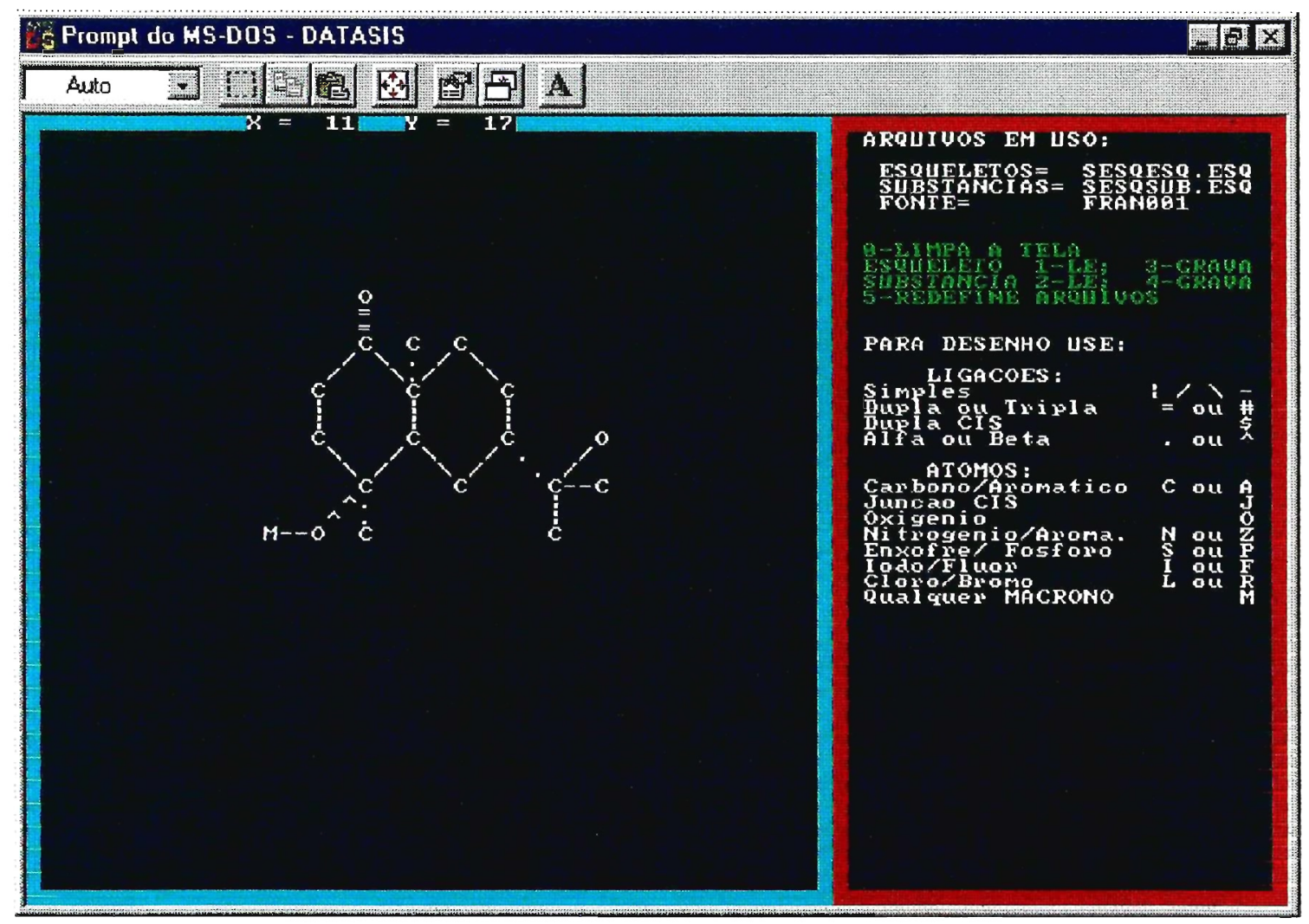

Figura 11. Tela de desenho do programa DATASIS.

Após o desenho da substância, o programa numera todos os átomos da estrutura e pede a confirmação da mesma. Em caso afirmativo, ele apresenta o vetor completo, o número de bytes usados na compactação e o número de oxidação da substância (figura 12). O programa também calcula automaticamente o identificador matemático. Em seguida são solicitados os dados obrigatórios (classe e esqueleto da substância, referência bibliográfica e, se existirem, os dados optativos (família, gênero e espécie). 


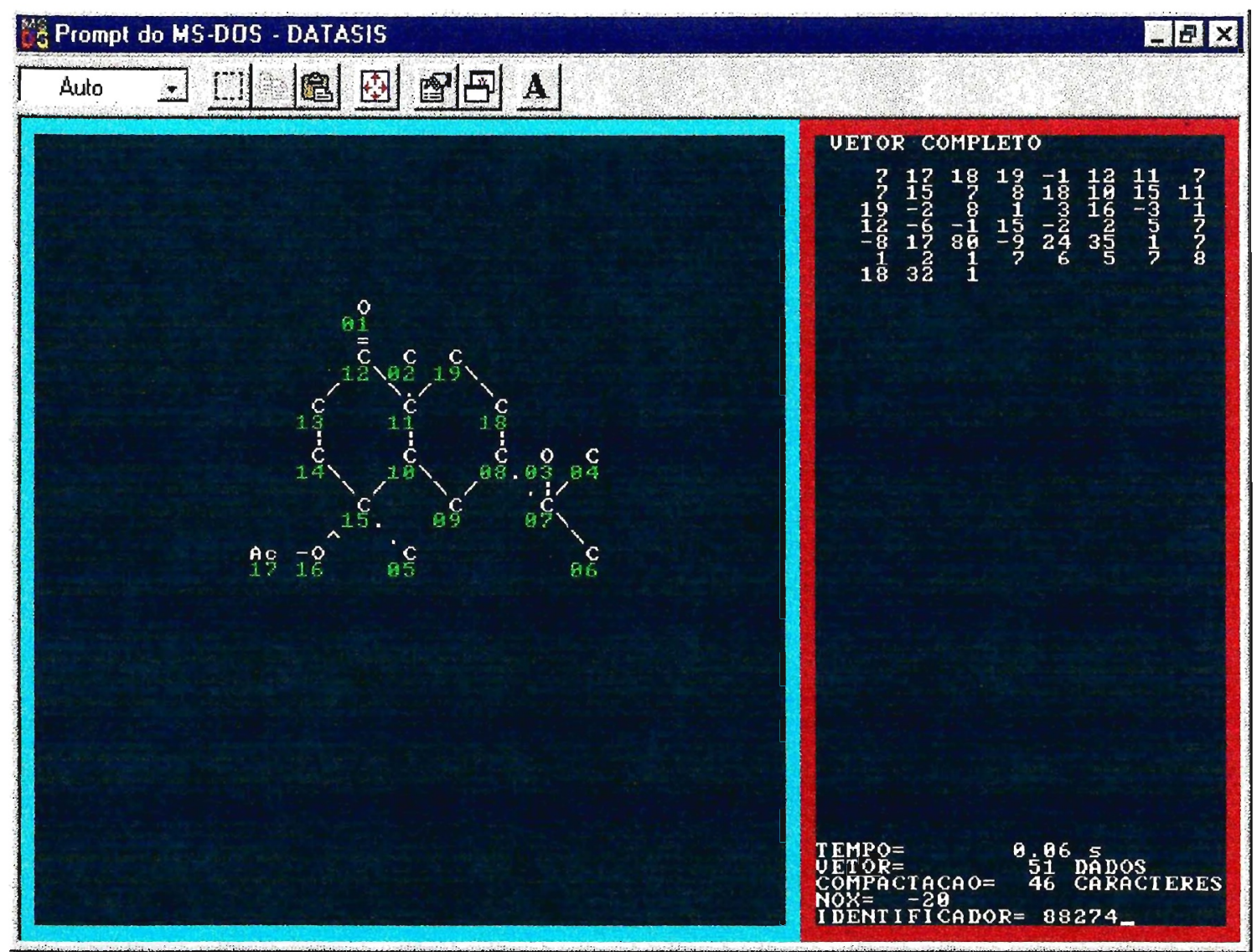

Figura 12. Tela de codificação do programa DATASIS.

Logo depois, é necessária a arquiteturaçăo do banco-fonte, utilizando-se o programa ARQUISIS. Por arquiteturação, entende-se a distribuição dos dados do banco fonte em bancos interligados, que contêm apenas um tipo de dado (figura 13). A arquiteturação é necessária para que sejam introduzidos os dados físico-químicos como RMN ${ }^{13} \mathrm{C}$ e outros, tais como, o vetor biogenético, a parte da planta da qual o sesquiterpeno foi isolado, local e época de coleta. Isto é feito através da opção 4.

A opção 6 permite corrigir as informações contidas nos bancos-fonte. No entanto, os dados corrigidos nos bancos-fonte continuam incorretos nos bancos arquiteturados, sendo sempre necessária uma rearquiteturação do sistema após alguma correção no banco-fonte.

A opção 7 permite eliminar definitivamente uma substância do banco de dados, após a sua desativação através da opção 6 . Isto normalmente é necessário quando houver algum erro no desenho da estrutura. 


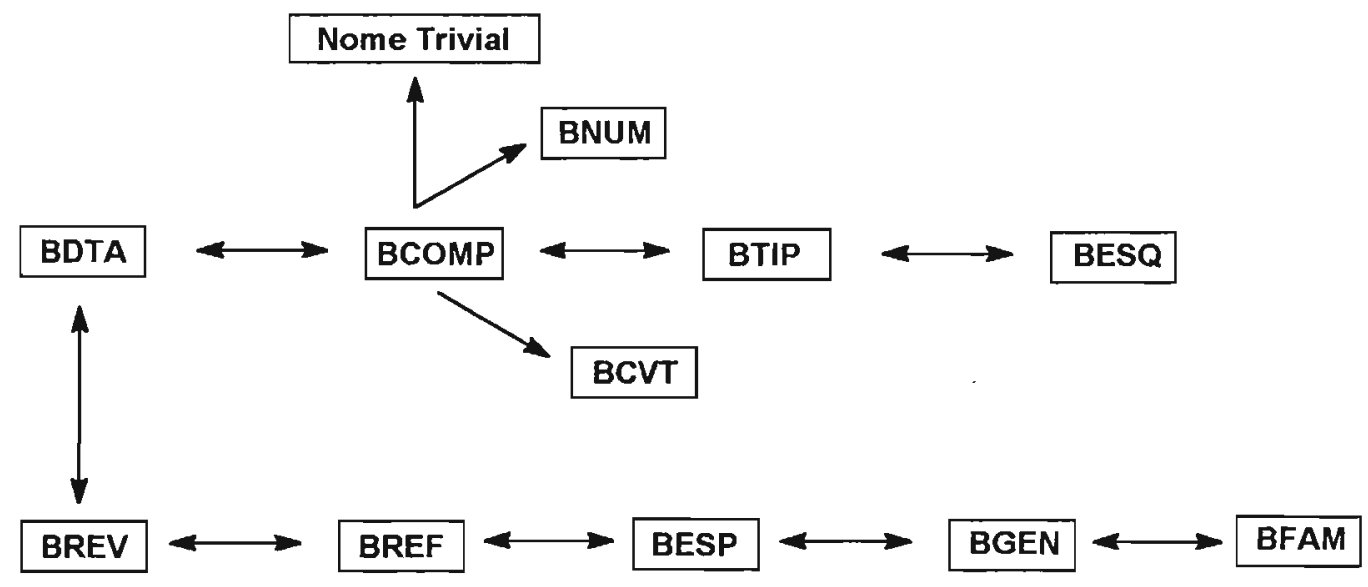

Figura 13. Esquema dos bancos arquiteturados do SISTEMAT.

BANCO

BNUM

BTIP

BESQ

BREF

BREV

BESP

BGEN

BFAM

BCOMP

BDTA
CONTEÚDO

Número matemático criado para cada substância

Classe das substâncias e tipos de dados

Nome do esqueleto

Referências bibliográficas

Nome das revistas

Espécies

Gêneros

Familias

Código das substâncias

Dados físico-químicos 


\subsection{PROGRAMAS APLICATIVOS}

Os programas aplicativos são realmente a parte "inteligente" do SISTEMAT, permitindo a análise dos dados presentes no sistema e a obtenção de informações importantes para o processo de elucidação estrutural. Através dos dados de $\mathrm{RMN}{ }^{13} \mathrm{C}$, EM e dados botânicos, estes programas podem caracterizar o tipo de esqueleto de uma substância desconhecida.

\subsection{1- Os programas SISLIST e REFERENC}

O programa SISLIST fornece dois tipos de listagem sobre o banco de dados. Uma delas apresenta o total de substâncias em cada classe e a distribuição destas substâncias em cada esqueleto. A outra apresenta informações botânicas, ou seja, para cada família são apresentadas as ocorrências de cada espécie e a soma de ocorrências por gênero e familia. Nas tabelas 2 e 3 são mostradas as listagens obtidas através deste aplicativo para o banco de sesquiterpenos. A tabela 2 mostra o número total de sesquiterpenos no banco de dados e o número de substâncias por esqueleto e, a tabela 3 , o número de sesquiterpenos presentes, por exemplo, na família Celastraceae por gênero e espécie.

O programa REFERENC fornece as referências bibliográficas usadas na criação do banco de dados, listando o nome da revista, ano, página e o número de substâncias ou ocorrências botânicas obtidas nesta referência. No entanto, o programa não fornece as referências bibliográficas dos compostos que não apresentam dados botânicos. 
Tabela 2. Listagem dos esqueletos e número de ocorrências.

\begin{tabular}{|c|c|c|c|}
\hline ise & SESQUITERPENO & 2306 & \\
\hline 1. - & EUDESMANO & 349 & compostos \\
\hline $2 .-$ & CADINANO & 124 & compostos \\
\hline 3.- & 12,13 -DINOR-CADINANO & 1 & compostos \\
\hline 4. - & 15-NOR-CADINANO & 1 & compostos \\
\hline 5.- & DRIMANO & 58 & compostos \\
\hline 6.- & EREMOFILANO & 68 & compostos \\
\hline 7.- & GUAIANO & 93 & compostos \\
\hline 8. - & $11,12,13$-TRINOR-EUDESMANO & 5 & compostos \\
\hline 9.- & AROMADENDRANO & 57 & compostos \\
\hline $10 .-$ & $15(->1)$-EUDESMANO & 5 & compostos \\
\hline $11 .-$ & $14(->3)-$ DRIMANO & 9 & compostos \\
\hline $12 .-$ & AFRICANO & 6 & compostos \\
\hline $13 .-$ & II-NOR-DRIMANO & 3 & compostos \\
\hline $14 .-$ & 11NOR-14(->3)-DRIMANO & $I$ & compostos \\
\hline $15 .-$ & 12 -NOR-DRIMANO & 1 & compostos \\
\hline $16 .-$ & CAROTANO & 113 & compostos \\
\hline $17 .-$ & 14-NOR-GUAIANO & 1 & compostos \\
\hline $18 .-$ & GERMACRANO & 65 & compostos \\
\hline $19 .-$ & ARISTOLANO & 19 & compostos \\
\hline $20 .-$ & BRAS ILANO & 3 & compostos \\
\hline $21 .-$ & BISABOLANO & 158 & compostos \\
\hline $22 .-$ & ACORANO & 4 & compostos \\
\hline $23 .-$ & EPICUBEBOL & 4 & compostos \\
\hline 24 . - & $14(->6)$ - FURANOEREMOFILANO & 10 & compostos \\
\hline $25 .-$ & 4, 5-SECO-FURANOEREMOF ILANO & 3 & compostos \\
\hline $26 .-$ & 4, 5-SECO-FUROEREMOF II_ANO & 1 & compostos \\
\hline $27 .-$ & ELEMANO & 20 & compostos \\
\hline $28 .-$ & LONGIPINANO & 41 & compostos \\
\hline $29 .-$ & 15-NOR-PINGUISANO & 3 & compostos \\
\hline $30 .-$ & 4, 5-SECO-GUAIANO & 4 & compostos \\
\hline $31 .-$ & CIPERANO & 12 & compostos \\
\hline $32 .-$ & CEDRANO & 10 & compostos \\
\hline $33 .-$ & $14(->1)$-MALIANO & 1 & compostos \\
\hline $34 .-$ & $1,10-$ SECO-EUDESMANO & 1 & compostos \\
\hline $35 .-$ & PINGUISANO & 12 & compostos \\
\hline 36. & ISOCAPNELANO & 3 & compostos \\
\hline $37 .-$ & LACTARANO & 14 & compostos \\
\hline $38 .-$ & 2, 3-SECO-AROMADENDRANO & 20 & compostos \\
\hline $39 .-$ & CARIOFILANO & 35 & compostos \\
\hline $40 .-$ & 14-NOR-CARIOFILANO & 1 & compostos \\
\hline 41.- & $4,5-S E C O-C A R I O F$ ILANO & 4 & compostos \\
\hline $42 .-$ & 14-NOR-EREMOFILANO & 1 & compostos \\
\hline $43 .-$ & 13-NOR-EREMOF ILANO & 2 & compostos \\
\hline $44 .-$ & 14-NOR-1,2-SECO-CADINANO & 2 & compostos \\
\hline $45 .-$ & 1,2 -SECO-CADINANO & 3 & compostos \\
\hline 46.- & BULATANO & 5 & compostos \\
\hline $47 .-$ & PROTOILUDANO & 41 & compostos \\
\hline $48 .-$ & 8,9 -SECO-AFR ICANO & 1 & compostos \\
\hline $49 .-$ & 15-NOR-EUDESMANO & 6 & compostos \\
\hline $50 .-$ & FUROEREMOFILANO & 1 & compostos \\
\hline $51 .-$ & FARNESANO & 90 & compostos \\
\hline & SII & & \\
\hline
\end{tabular}


Tabela 2. Continuação

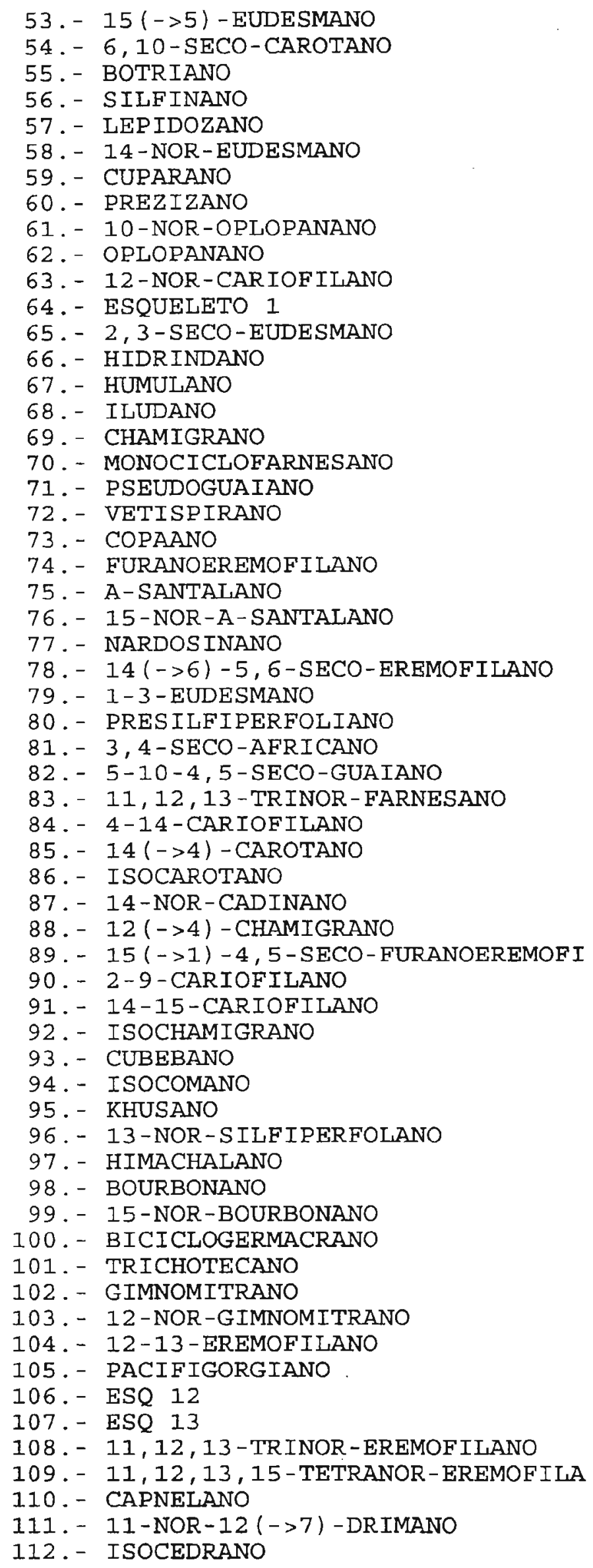

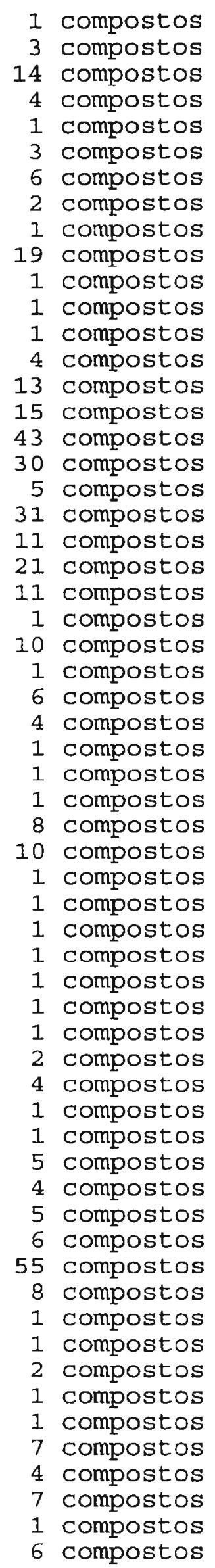


Tabela 2. Continuação

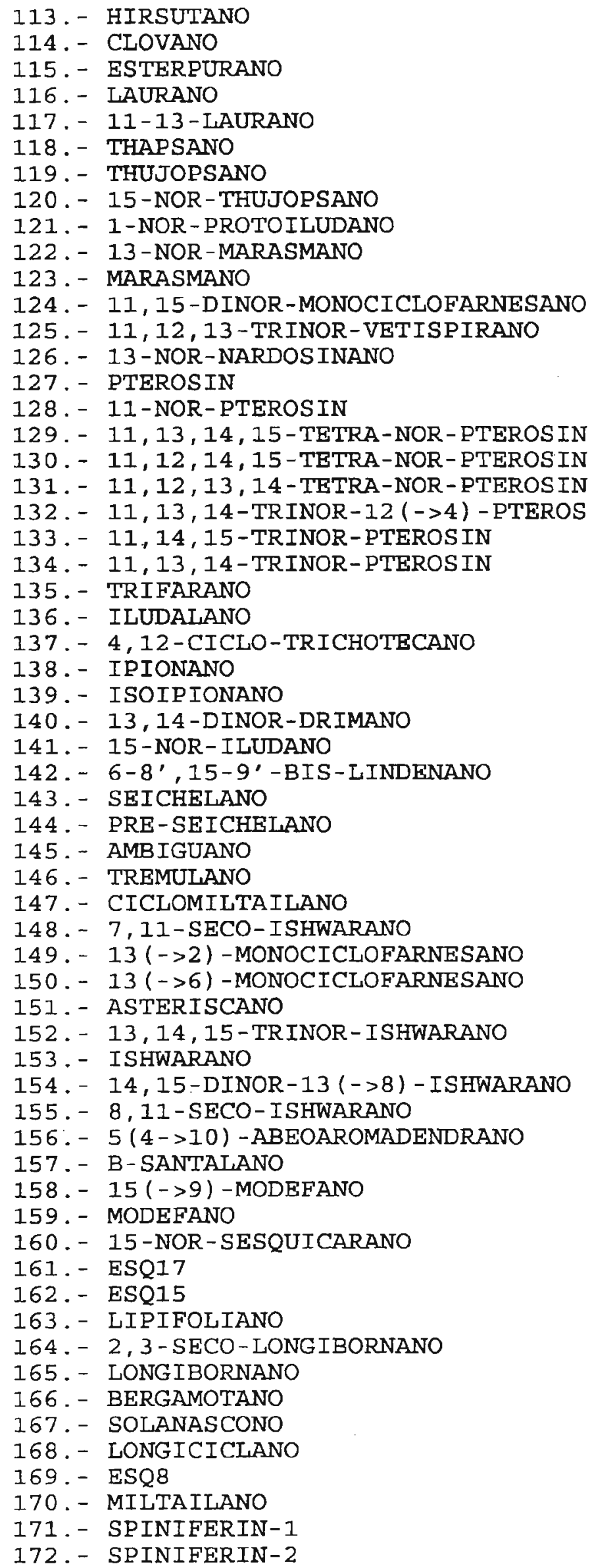

6 compostos

7 compostos

3 compostos

8 compostos

1 compostos

5 compostos

10 compostos

I compostos

1 compostos

2 compostos

3 compostos

2 compostos

2 compostos

3 compostos

6 compostos

11 compostos

1 compostos

1 compostos

1 compostos

1 compostos

1 compostos

1 compostos

7 compostos

2 compostos

1 compostos

2 compostos

1 compostos

1 compostos

2 compostos

2 compostos

1 compostos

4 compostos

1 compostos

7 compostos

2 compostos

1 compostos

13 compostos

1 compostos

4 compostos

1 compostos

3 compostos

1 compostos

1 compostos

1 compostos

2 compostos

2 compostos

1 compostos

1 compostos

1 compostos

1 compostos

4 compostos

1 compostos

1 compostos

2 compostos

1 compostos

I compostos

1 compostos

1 compostos

2 compostos

1 compostos 
Tabela 2. Continuação

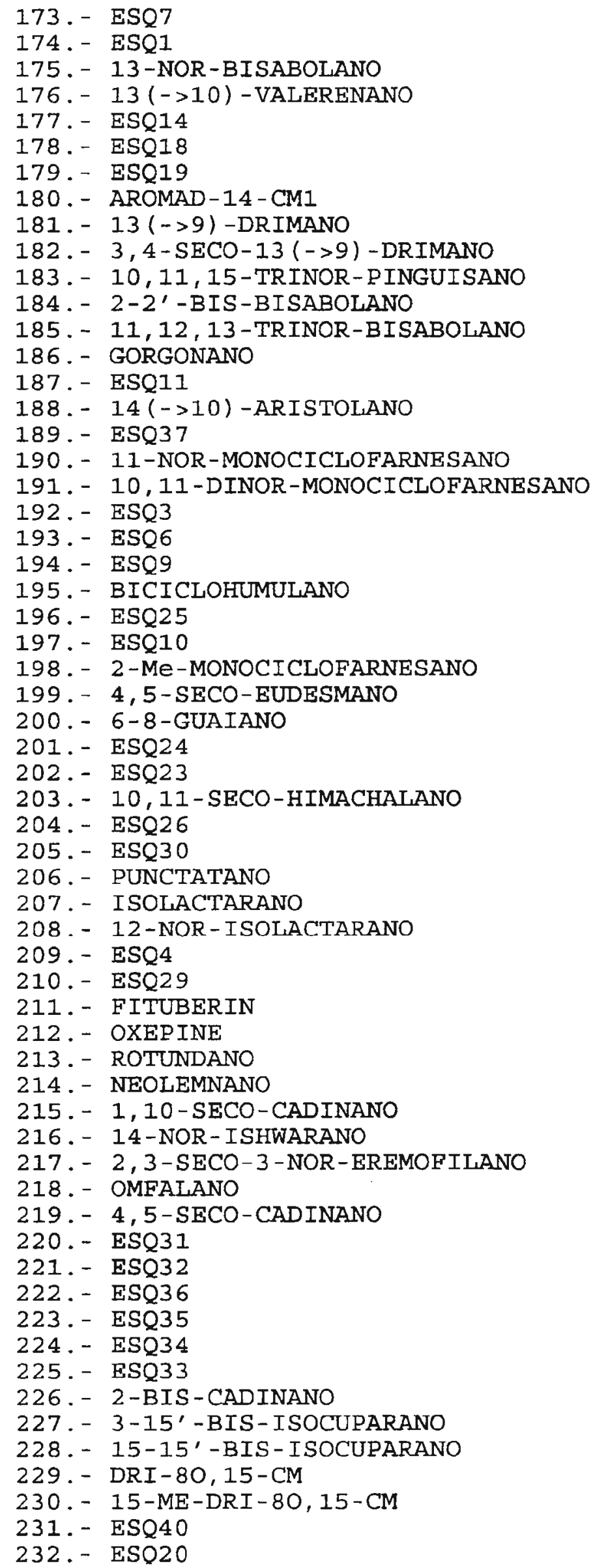

1 compostos

3 compostos

2 compostos

2 compostos

4 compostos

1 compostos

3 compostos

1 compostos

1 compostos

2 compostos

3 compostos

3 compostos

1 compostos

2 compostos

3 compostos

1 compostos

3 compostos

2 compostos

11 compostos

1 compostos

6 compostos

1 compostos

1 compostos

1 compostos

1 compostos

1 compostos

1 compostos

2 compostos

2 compostos

1 compostos

1 compostos

5 compostos

2 compostos

1 compostos

1 compostos

1 compostos

1 compostos

1 compostos

2 compostos

4 compostos

1 compostos

2 compostos

1 compostos

1 compostos

1 compostos

1 compostos

2 compostos

5 compostos

4 compostos

1 compostos

1 compostos

2 compostos

1 compostos

1 compostos

1 compostos

1 compostos

7 compostos

1 compostos

1 compostos

10 combostos 
Tabela 2. Continuação

\begin{tabular}{|c|c|c|c|}
\hline 233.- & ESQ21 & 2 & compostos \\
\hline $234 .-$ & ESQ2 2 & 1 & compostos \\
\hline 235 .- & ESQ38 & 3 & compostos \\
\hline $36 .-$ & ESQ41 & 2 & compostos \\
\hline $237 .-$ & ESQ5 & 2 & compostos \\
\hline $238 .-$ & $11,12,13-T R I N O R-E L E M A N O$ & 6 & compostos \\
\hline $239 .-$ & 12,13 -DINOR-ELEMANO & 1 & compostos \\
\hline 240 .- & $13(->7)$-ELEMANO & 1 & compostos \\
\hline $241 .-$ & 13-NOR-ELEMANO & 5 & compostos \\
\hline 242 . - & $\operatorname{ESQ} 28$ & 3 & compostos \\
\hline $243 .-$ & ESQ27 & 6 & compostos \\
\hline $244 .-$ & $11,12,14$-TRINOR-OXEPINA & 1 & compostos \\
\hline 245 . - & 1,2-DINOR-FARNESANO & 1 & compostos \\
\hline $246 .-$ & ESQ42 & 1 & compostos \\
\hline $247 .-$ & ESQ43 & 1 & compostos \\
\hline 248.- & ESQ44 & 1 & compostos \\
\hline $249 .-$ & $5-10,4,5-S E C O-G U A I A N O$ & 1 & compostos \\
\hline $250 .-$ & 1,10 -SECO-AROMADENDRANO & 1 & compostos \\
\hline 251.- & 2-10-HIMACHALANO & 3 & compostos \\
\hline 252 . - & 14-4-CARIOF ILANO & 1 & compostos \\
\hline 253.- & ESQ45 & 1 & compostos \\
\hline 254 .- & MALIANO & 2 & compostos \\
\hline 255 . - & EUD - 3,40-CM & 1 & compostos \\
\hline $256 .-$ & VALERENANO & 4 & compostos \\
\hline $257 .-$ & ALIACANO & 4 & compostos \\
\hline 258 . - & $9,10-$ SECO-DRIMANO & 3 & compostos \\
\hline $259 .-$ & FURODISANO & 2 & compostos \\
\hline $260 .-$ & 8,9 -SECO-LACTARANO & 1 & compostos \\
\hline $261 .-$ & ESQ46 & 1 & compostos \\
\hline $262 .-$ & ESQ47 & 1 & compostos \\
\hline $263 .-$ & ESQ56 & 1 & compostos \\
\hline 264 .- & ESQ4 8 & 1 & compostos \\
\hline 265 .- & ESQ49 & 1 & compostos \\
\hline $266 .-$ & ESQ50 & 1 & compostos \\
\hline 267 . - & ESQ51A & 1 & compostos \\
\hline $268 .-$ & ESQ51B & 1 & compostos \\
\hline $269 .-$ & ESQ52 & 3 & compostos \\
\hline 270 .- & ESQ53 & 1 & compostos \\
\hline $271 .-$ & ESQ54 & 1 & compostos \\
\hline $272 .-$ & CHILOSCIFANO & 2 & compostos \\
\hline 273.- & $15(->4)$-LONGIBORNANO & 1 & compostos \\
\hline $274 .-$ & ESQ58 & 1 & compostos \\
\hline 275 .- & ESQ57 7 & 1 & compostos \\
\hline 276 . - & $5(->4)$ - LACTARANO & 2 & compostos \\
\hline $277 .-$ & $14(->6)$-CUPARANO & 1 & compostos \\
\hline 278 . - & $1(11->10)-$ ABEOMARASMANO & 1 & compostos \\
\hline $279 .-$ & PROBOTRIANO & 1 & compostos \\
\hline $280 .-$ & ESQ59 & 1 & compostos \\
\hline 281.- & ESQ60 & 7 & compostos \\
\hline $282 .-$ & $12,13,15$-TRINOR-HIRSUTANO & 1 & compostos \\
\hline $283 .-$ & ESQ62 & 1 & compostos \\
\hline $284 .-$ & FOMANOS IN & 1 & compostos \\
\hline $285 .-$ & ESQ69 & 1 & compostos \\
\hline $286 .-$ & 5-14-CARIOFILANO & 2 & compostos \\
\hline $287 .-$ & 12-14'-BIS-14(->6)-7,8-FURANOE & 1 & compostos \\
\hline $28 \varepsilon$ & ESQ55 & 1 & compostos \\
\hline
\end{tabular}


Tabela 3. Listagem das ocorrências botânicas obtidas pelo Sislist para a família Celastraceae.

\begin{tabular}{|c|c|c|}
\hline \multicolumn{3}{|l|}{$\begin{array}{c}\text { Familia : CELASTRACEAE } \\
\text { Gênero : MAYTENUS }\end{array}$} \\
\hline \multicolumn{3}{|l|}{$\begin{array}{l}\text { Espécie: BOARIA } \\
\text { Total do Gênero : MAYTENUS } \\
\text { Gênero: TRIPTERYGIUM }\end{array}$} \\
\hline & Total & $\begin{array}{l}=16 \\
=16\end{array}$ \\
\hline $\begin{array}{l}\text { Espécie : ROSTHORNIANUS } \\
\text { Espécie : ANGULATUS } \\
\text { Espécie : GEMMATUS } \\
\text { Espécie : PANICULATUS }\end{array}$ & $\begin{array}{l}\text { Total } \\
\text { Total } \\
\text { Total } \\
\text { Total }\end{array}$ & $\begin{array}{l}=2 \\
=8 \\
=6 \\
=1 \\
=17\end{array}$ \\
\hline \multicolumn{3}{|l|}{$\begin{array}{l}\text { Gênero : EUONYMUS } \\
\text { Espécie : BUNGEANL }\end{array}$} \\
\hline $\begin{array}{l}\text { Espécie : SIEBOLDIANUS } \\
\text { Espécie : SACHALIENSIS } \\
\text { Espécie : NANUS }\end{array}$ & $\begin{array}{l}\text { Total } \\
\text { Total } \\
\text { Tota }\end{array}$ & $\begin{array}{l}=1 \\
=1 \\
=1\end{array}$ \\
\hline \multicolumn{3}{|l|}{ Total do gênero : EUONYMUS } \\
\hline \multicolumn{2}{|l|}{ Total da família : CELASTRACEAE } & $=41$ \\
\hline
\end{tabular}

\subsection{2- Os programas SISBOTA e SISOCBOT ${ }^{19,25}$}

Estes programas permitem a análise dos dados botânicos e requerem como dado de entrada obrigatório o nome da família que se quer pesquisar, sendo o gênero e a espécie optativos. Como resultado, fornecem uma listagem do número de ocorrências de cada esqueleto na família e/ou gênero escolhidos.

Além da família, o SISBOTA também permite o uso de outras condições de procura como classe e/ou esqueleto, massa molecular, índice de oxidação, fórmula bruta e requisitos químicos, podendo fornecer oito tipos de informações :

1 -classe e/ou esqueleto

2- massa molecular

3- índice de oxidação

4- fórmula bruta

5- dados botânicos completos

6- desenho da molécula

7- referência bibliográfica

8- dados físico-químicos 
Também podem ser obtidos os histogramas de uma família ou gênero escolhido, contra índice de oxidação e/ou esqueleto e/ou classe. Os histogramas são recursos interessantes apenas se o sistema apresentar uma base de dados desenvolvida para análise quimiossistemática, isto é, se existir uma amostragem representativa de dados botânicos acerca de um determinado grupo taxonômico.

O programa SISOCBOT fornece a porcentagem dos esqueletos presentes em cada família e gênero, sendo utilizado também como mais uma ferramenta para a previsão do tipo de esqueleto. Para exemplicar o seu uso, o programa foi aplicado em 8 famílias e os dados obtidos encontram-se na tabela 4, onde são mostrados os dois esqueletos com maior número de ocorrências nessas familias.

Tabela 4. Esqueletos de sesquiterpenos mais frequentes em 8 famílias.

\begin{tabular}{|l|ll|}
\hline FAMÍLIA & \multicolumn{2}{|c|}{ ESQUELETOS } \\
\hline Solanaceae & cadinano $(45.0 \%)$ & eremofilano(12.5\%) \\
\hline Celastraceae & eudesmano $(100.0 \%)$ \\
\hline Asteraceae & eudesmano $(26.2 \%)$ & bisabolano (9.2\%) \\
\hline Cupressaceae & eudesmano (16.0) & cadinano (56.0\%) \\
\hline Meliaceae & cadinano $(41.0 \%)$ & carotano $(33.3 \%)$ \\
\hline Canellaceae & drimano $(100 \%)$ & \\
\hline Umbelliferae & carotano $(65.5 \%)$ & guaiano $(8.6 \%)$ \\
\hline
\end{tabular}




\subsection{3- SISCONST $T^{34}$}

Este programa foi desenvolvido para auxiliar o químico na determinação estrutural de produtos naturais, utilizando os dados de $\mathrm{RMN}{ }^{13} \mathrm{C}$ (deslocamentos químicos e multiplicidades). É um dos programas mais importantes do SISTEMAT, pois fornece o tipo de esqueleto mais provável para uma substância-teste, grandes subestruturas compativeis com o espectro-problema e a atribuição dos sinais de RMN ${ }^{13} \mathrm{C}$.

O SISCONST confronta o espectro questionado com todos os espectros pertencentes à base de dados. Se um sinal do espectro questionado está presente, por exemplo, no espectro da primeira substância do banco e tem a mesma multiplicidade, os deslocamentos químicos dos carbonos vizinhos interligados são então confrontados com os do espectro-problema. Este processo se repete até que sejam obtidos os maiores fragmentos das substâncias do banco que apresentem deslocamentos químicos compativeis com os dados do espectro-problema.

Para as pesquisas de subestrutura e esqueleto foram fixados os intervalos de erro de 1,0 e 5,0 ppm, respectivamente. Se a pesquisa não é bem sucedida, os intervalos são aumentados de acordo com um gradiente de 0,5 e 1,0 ppm, respectivamente. Caso o intervalo atinja um valor maior do que $3,0 \mathrm{ppm}$ para estruturas e maior do que 10,0 ppm para esqueletos, o sistema pára e não há previsão para esqueleto e/ou subestrutura. Na busca dos esqueletos mais prováveis, as substâncias escolhidas devem ter uma subestrutura contendo pelo menos metade do número total de carbonos.

O usuário deve fornecer ao programa, além dos deslocamentos químicos e multiplicidades, as seguintes informações:

- O número mínimo de átomos para a subestrutura proposta.

- A faixa de erro admitida pelo programa, geralmente 1,0 ppm.

- O gradiente, que permite o aumento automático da faixa de erro, caso não seja encontrada uma resposta com os parâmetros iniciais;

- O espaço da pesquisa, ou seja, se esta deve ser feita sobre os compostos de toda a classe ou de um único esqueleto. 
Para exemplificar o uso deste programa foi utilizado o espectro de $\mathrm{RMN}{ }^{13} \mathrm{C}$ do sesquiterpeno de esqueleto cadinano (figura 14), isolado de Artemisia chamaemelifolia (Compositae) $^{35}$, que não consta do banco de dados. Foi exigido um número mínimo de 7 sinais coincidentes (subestruturas com menos de 7 sinais não serão consideradas) $e$ um gradiente de $1,0 \mathrm{ppm}$. Inicialmente, o programa apresenta a probabilidade da substância pertencer a um determinado tipo de esqueleto (tabela 5) e lista apenas a subestrutura A (figura 15), com a atribuição dos deslocamentos químicos (tabela 6). Em seguida, a procura é encerrada,sendo apresentada ao usuário a opção de continuar com a mesma substância. Em caso afirmativo, é listado o espectro digitado e pode-se escolher quais os deslocamentos a serem usados para uma nova pesquisa. Neste caso, a faixa de erro foi ampliada para 3,0 ppm e foram mantidos apenas os deslocamentos químicos não atribuídos, para os quais foi proposta a subestrutura $B$ (figura 15) com a atribuição apresentada na tabela 6 . Os deslocamentos químicos atribuídos aos carbonos 13 e 14 encontram-se invertidos quando comparados com os dados da literatura. Isto pode refletir um erro nos dados da literatura ou mesmo um erro presente na base de dados.

A sobreposição das estruturas A e B, juntamente com a informação de que o esqueleto mais provável é um cadinano, nos conduz à estrutura do sesquiterpeno da figura 14.

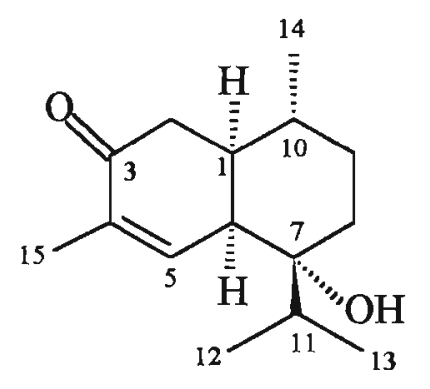

Figura 14. Estrutura do sesquiterpeno usado para teste do programa SISCONST. 
Tabela 5. Análise do esqueleto mais provável para uma dada substância, utilizando-se o programa SISCONST.

\begin{tabular}{|l|c|}
\hline Esqueleto & Probabilidade \\
\hline \hline Cadinano & 78.4 \\
Esqueleto-25 & 9.1 \\
Vetispirano & 0.0 \\
Isocomano & 0.0 \\
Guaiano & 2.3 \\
Bisabolano & 1.1 \\
Germacrano & 1.7 \\
Aromadendrano & 1.7 \\
Outros & 5.6 \\
\hline
\end{tabular}

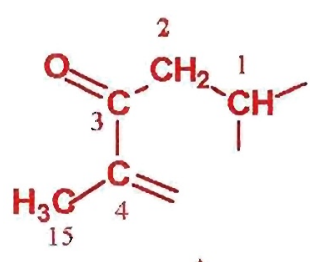

A

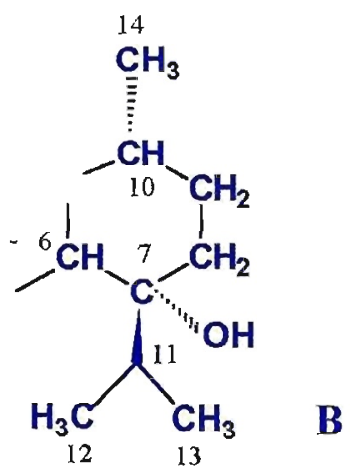

B

Figura 15. Subestruturas propostas pelo programa SISCONST.

Tabela 6. Dados de $\mathrm{RMN}{ }^{13} \mathrm{C}$ do sesquiterpeno da figura 14 associados aos deslocamentos químicos atribuídos às subestruturas $\mathrm{A}$ e $\mathrm{B}$ pelo programa SISCONST.

\begin{tabular}{rccc}
\hline C & Literatura & A & B \\
\hline 1 & 40.8 & 40.0 & -- \\
2 & 42.4 & 43.0 & --- \\
3 & 199.6 & 199.8 & --- \\
4 & 136.7 & 135.8 & -- \\
5 & 142.9 & --- & -- \\
6 & 45.0 & -- & 44.0 \\
7 & 74.4 & --- & 74.9 \\
8 & 32.5 & -- & 31.7 \\
9 & 29.8 & -- & 30.1 \\
10 & 28.1 & -- & 27.1 \\
11 & 33.0 & --- & 32.8 \\
12 & $16.1 *$ & --- & 15.6 \\
13 & $15.7^{*}$ & --- & 19.5 \\
14 & 19.3 & --- & 16.0 \\
15 & 16.1 & 16.1 & -- \\
\hline
\end{tabular}

*deslocamentos químicos intercambiáveis 


\subsection{4- O programa $\mathrm{C} 13 \mathrm{MACH}$}

O C13MACH, assim como o SISCONST, é um sistema que prevê, a partir dos dados do espectro experimental de $\mathrm{RMN}{ }^{13} \mathrm{C}$ (sinal e multiplicidade), o tipo de esqueleto de um determinado produto natural, fornecendo também as estruturas existentes no banco de dados que têm espectros semelhantes ao da amostra analisada. Este sistema é constituído de dois programas ${ }^{28}$ :

a) O primeiro é o programa C13CR que extrai do SISTEMAT os dados necessários para análise e cria um novo banco para o $\mathrm{C} 13 \mathrm{MACH}$ propriamente dito. Este programa lista os tipos de dados físico-químicos disponiveis para que o usuário escolha os códigos dos dados de interesse.

b) O segundo é o programa de procura, denominado $\mathrm{C13MACH}$, que utiliza dados de $\mathrm{RMN}{ }^{13} \mathrm{C}$ e trabalha com um algoritmo de comparação. Este programa confronta os sinais do espectro questionado com os dados do banco e verifica o índice de semelhança entre eles, o qual é calculado a partir do número de sinais do espectro analisado que estão presentes em cada uma das substâncias do banco. Quanto maior o índice de semelhança, maior será a probabilidade do espectro analisado pertencer a uma substância com o mesmo esqueleto daquela presente no banco.

O programa fornece como dados de saída as estruturas das substâncias onde ocorre alguma coincidência espectral, 0 indice de semelhança e a porcentagem de ocorrência desses deslocamentos químicos nos vários esqueletos do banco de dados. No entanto, ele não requer a condição de que os átomos de carbonos responsáveis pelos deslocamentos químicos, dentro da faixa de erro especificada, estejam interligados. Isto pode conduzir a um resultado impreciso.

Os dados solicitados ao usuário são os seguintes:

- a faixa de erro admitida pelo programa, ou seja, de quanto pode ser a diferença entre o deslocamento químico formecido e o deslocamento químico dos compostos presentes no banco.

- o número de compostos com melhores índices de semelhança que devem ser considerados para o cálculo da probabilidade de esqueleto. Este valor pode variar de acordo com a classe de compostos estudada. 
- os dados dos deslocamentos químicos e multiplicidades do espectro problema.

Este programa foi testado com o espectro de $\mathrm{RMN}{ }^{13} \mathrm{C}$ do mesmo sesquiterpeno utilizado no exemplo do programa SISCONST (figura14), sendo apresentados na figura 16 os quatro sesquiterpenos do banco com maior índice de similaridade. A tabela 7 mostra os esqueletos mais prováveis fornecidos pelo $\mathrm{C} 13 \mathrm{MACH}$, com base na análise dos 10 sesquiterpenos com melhor índice de similaridade ${ }^{36}$.

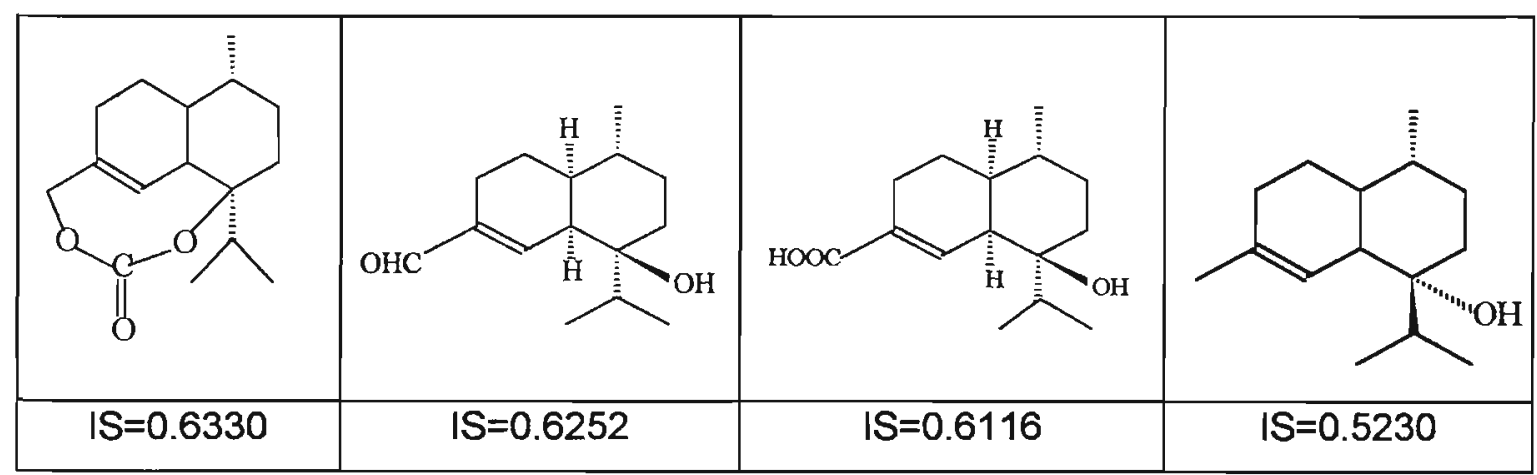

Figura 16. Sesquiterpenos com os melhores indices de similaridade.

Tabela 7. Análise dos esqueletos mais prováveis fornecidos pelo programa C13MACH.

\begin{tabular}{|l|c|}
\hline Esqueleto & Porcentagem \\
\hline Cadinano & $91 \%$ \\
\hline Eudesmano & $9 \%$ \\
\hline
\end{tabular}




\subsection{5- O programa TIPCARB}

Este programa apresenta uma listagem com o padrão de substituição de cada átomo de um determinado esqueleto, de acordo com a numeração padronizada. Deste modo, pode-se verificar quais são as posições mais ou menos oxidadas em um esqueleto. Estas informações são de grande relevância porque elas podem guiar o usuário na busca de regras heurísticas.

Como exemplo, são apresentados, na tabela 8 , os dados fornecidos pelo programa para o esqueleto drimano (figura 17).

Tabela 8. Listagem fornecida pelo programa TIPCARB para o esqueleto drimano.

\begin{tabular}{|c|rrrrrrrrrrr|}
\hline $\mathrm{C}$ & $\mathrm{CH}_{3}$ & $\mathrm{CH}_{2}$ & \multicolumn{1}{c}{$\mathrm{CH}$} & \multicolumn{1}{c}{$\mathrm{C}$} & $\mathrm{CH}_{2}=$ & $\mathrm{CH}=$ & $\mathrm{C}=$ & $\mathrm{A}$ & $\mathrm{B}$ & $\mathrm{C}$ & $\mathrm{D}$ \\
\hline 1 & 0 & 57 & 1 & 0 & 0 & 0 & 0 & 0 & 0 & 0 & 0 \\
2 & 0 & 57 & 1 & 0 & 0 & 0 & 0 & 0 & 0 & 0 & 0 \\
3 & 0 & 31 & 27 & 0 & 0 & 0 & 0 & 0 & 0 & 0 & 0 \\
4 & 0 & 0 & 0 & 58 & 0 & 0 & 0 & 0 & 0 & 0 & 0 \\
5 & 0 & 0 & 57 & 0 & 0 & 0 & 1 & 0 & 0 & 0 & 0 \\
6 & 0 & 49 & 4 & 0 & 0 & 1 & 4 & 0 & 0 & 0 & 0 \\
7 & 0 & 21 & 2 & 0 & 0 & 35 & 0 & 0 & 0 & 0 & 0 \\
8 & 0 & 0 & 0 & 3 & 0 & 0 & 55 & 0 & 0 & 0 & 0 \\
9 & 0 & 0 & 51 & 6 & 0 & 0 & 1 & 0 & 0 & 0 & 0 \\
10 & 0 & 0 & 0 & 58 & 0 & 0 & 0 & 0 & 0 & 0 & 0 \\
11 & 1 & 35 & 8 & 0 & 0 & 12 & 2 & 0 & 0 & 0 & 0 \\
12 & 16 & 5 & 6 & 0 & 19 & 11 & 1 & 0 & 0 & 0 & 0 \\
13 & 58 & 0 & 0 & 0 & 0 & 0 & 0 & 0 & 0 & 0 & 0 \\
14 & 58 & 0 & 0 & 0 & 0 & 0 & 0 & 0 & 0 & 0 & 0 \\
15 & 52 & 4 & 0 & 0 & 0 & 0 & 2 & 0 & 0 & 0 & 0 \\
\hline
\end{tabular}
A: HC tripla
B: C tripla
C: $\mathrm{CH}$ aromático
$\mathrm{D}:$ C aromático

A partir destes dados podemos verificar que nas 58 ocorrências deste esqueleto, o carbono 8 aparece 55 vezes como um carbono quaternário com uma ligação dupla, sendo que 35 vezes está ligado por dupla ligação ao carbono 7 e 19 vezes ao carbono 12. Os carbonos 5 e 9 aparecem, respectivamente, 57 e 51 vezes como carbonos terciários, sendo os carbonos 4 e 10 sempre do tipo quaternário, pela própria estrutura do esqueleto drimano. Tais dados nos permitem inferir quais são os átomos ou subestruturas constantes em um certo esqueleto. Estas serão importantes 
para o aplicativo PICKUIP, pois auxiliam na obtenção de faixas de deslocamentos químicos características de um determinado tipo de esqueleto.

No caso do esqueleto drimano, podemos utilizar para a pesquisa de sinais característicos a sequência dos carbonos $4,5,10,9$ e 8, bem como os carbonos 4, 10 , 7 e 8 ou 4, 10, 8 e 12. Estes últimos caracterizariam um subesqueleto do tipo 7endrimano ou $8(12)$ en-drimano.<smiles>CC1CCCC2(C)C1CCCC2(C)C</smiles>

Figura 17. Esqueleto drimano 


\subsection{6- O SISTEMA PICKUP}

Este sistema aplicativo realiza a tarefa de encontrar as faixas de desiocamentos químicos de ${ }^{13} \mathrm{C}$ (máximo e mínimo) para determinados grupos de átomos (subestruturas) que o químico considera prováveis de identificar um conjunto de substâncias de um determinado esqueleto, verificando também se estes deslocamentos são realmente característicos. Ele é constituído por quatro programas :

1) PICKCR2: este programa extrai do SISTEMAT apenas os dados necessários para a análise, criando uma nova rede de bancos com os deslocamentos químicos dos átomos de carbono de todas as substâncias ou por tipo de esqueleto. Isto diminui sensivelmente o tempo gasto na análise ${ }^{37}$.

2) SISPICK2 : é o sistema de procura propriamente dito. Ele escollhe, a partir da numeração biogenética e das subestruturas impostas pelo usuário, o conjunto de substâncias obedecendo a esses requisitos, fornecendo os deslocamentos químicos máximos e mínimos e as multiplicidades dos átomos das subestruturas ${ }^{28}$. Tais subestruturas podem ser escolhidas com base nos tipos de carbono que sofrem pouca variação no seu padrão de substituição. Para isto, utiliza-se o programa TIPCARB ou o próprio conhecimento do químico acerca de uma classe ou de um esqueleto.

Foram codificadas 17 subestruturas-padrão, mostradas na tabela 9 , onde cada letra pode representar qualquer átomo da tabela 10. Cada ligação também recebe um código segundo o tipo de ligação: ligação simples sem "configuração" ou aromática (código 01), dupla (código 02), tripla (código 03), simples de configuração alfa (código 04), simples de configuração beta (código 05).

Podem ser impostos até nove requisitos químicos, devendo-se informar sucessivamente, para cada um deles, os seguintes dados: o código da subestrutura, quantas vezes ela deve estar presente, os códigos dos grupos de átomos (na ordem) e das ligações (na ordem). As subestruturas mostradas na tabela 9 também podem ser interligadas, conduzindo a estruturas maiores. 
Tabela 9. Código das subestruturas utilizadas pelo programa PICKUP.

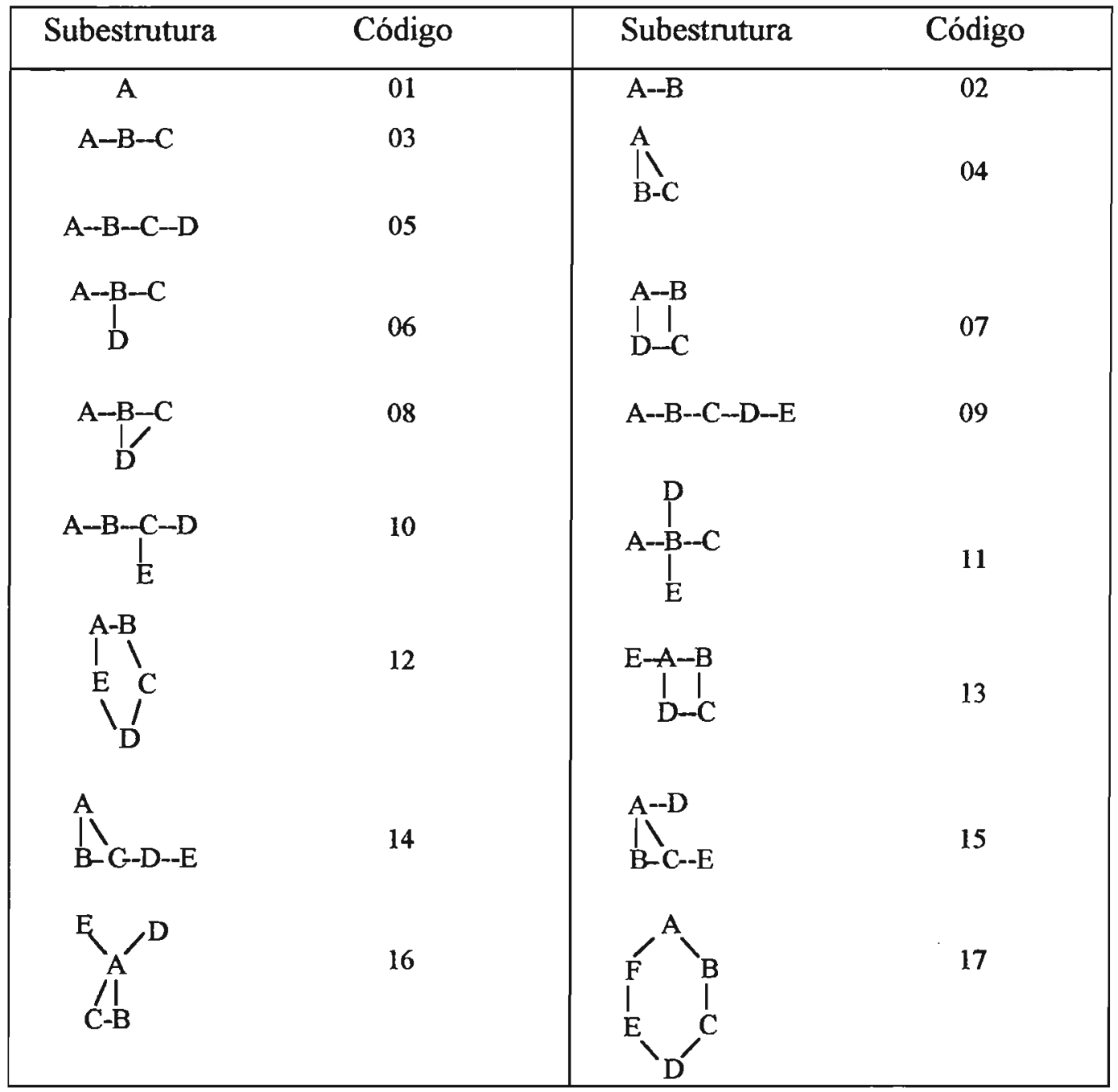

Tabela 10. Códigos que representam as letras nas subestruturas da Tabela 9.

\begin{tabular}{|cc|cc|cc|cc|}
\hline Átomo & Código & Átomo & Código & Átomo & Código & Átomo & Código \\
\hline$-\mathrm{CH}_{3}$ & 01 & $\equiv \mathrm{C}-$ & 09 & $-\mathrm{NH}-$ & 17 & $-\mathrm{Br}$ & 25 \\
$-\mathrm{CH}_{2}-$ & 02 & $\mathrm{HC}_{\mathrm{ar}}$ & 10 & $-\mathrm{N}-$ & 18 & $-\mathrm{I}$ & 26 \\
$-\mathrm{CH}-$ & 03 & $\mathrm{C} \mathrm{ar}$ & 11 & $=\mathrm{NH}$ & 19 & $-\mathrm{SH}$ & 27 \\
$-\mathrm{I}-$ & 04 & $=\mathrm{C}=$ & 12 & $=\mathrm{N}-$ & 20 & $-\mathrm{S}-$ & 28 \\
$\mathrm{I}$ & 05 & $=\mathrm{O}$ & 13 & $\equiv \mathrm{N}$ & 21 & $=\mathrm{S}$ & 29 \\
$=\mathrm{CH}_{2}$ & 05 & $-\mathrm{OH}$ & 14 & $\mathrm{~N}$ & 22 & $-\mathrm{S}=$ & 30 \\
$=\mathrm{C} H-$ & 06 & $-\mathrm{O}=$ & $-\mathrm{F}$ & 23 & $=\underset{\mathrm{S}}{\mathrm{I}}=$ & 31 \\
$=\mathrm{C}-$ & 07 & $-\mathrm{O}-$ & 15 & $-\mathrm{Cl}$ & 24 & $\mathrm{P}$ & 32 \\
$\equiv \mathrm{CH}$ & 08 & $-\mathrm{NH}_{2}$ & 16 & $-\mathrm{Cl}$ &
\end{tabular}


3) PICKRVR: Este programa verifica, em toda a base de dados, se as faixas de deslocamentos químicos obtidas pelo SISPICK2 são ou não são, ou em que grau, características de um determinado tipo de esqueleto, listando os compostos que apresentam aqueles requisitos espectrais. A informação sobre os tipos de carbonos também é necessária na análise.

Por exemplo, para análise do esqueleto drimano (figura 17), foi escolhida inicialmente a subestrutura abaixo (figura 18), obtida a partir dos dados da tabela 8 do programa TIPCARB.

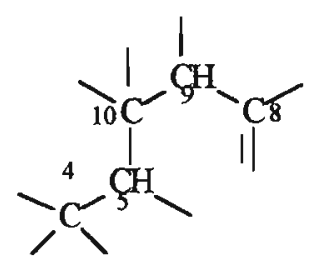

Figura 18. Subestrutura inferida a partir do programa TIPCARB para obtenção de deslocamentos químicos característicos de drimanos.

A subestrutura foi codificada ${ }^{37} \mathrm{e}$, através do uso do programa SISPICK2, foram obtidos os deslocamentos químicos máximos e mínimos de RMN ${ }^{13} \mathrm{C}$ desses cinco átomos. As faixas foram as seguintes: 40,7-29,6s (C-4); 56,0-43,5d (C-5); 42,2-32,9s (C-10); 60,7-50,5d (C-9) e 148,3-127,1s (C-8).

A seguir, através do programa PICKRVR, esses deslocamentos foram confrontados com todos os sesquiterpenos do banco de dados (2306 substâncias com 288 tipos de esqueletos). Foram listadas 53 substâncias, sendo 51 com esqueleto drimano e 2 com esqueleto longipinano, 0 que resulta num percentual de reconhecimento de $96,2 \%$. Deste modo, podemos dizer que existe uma alta probabilidade de que essas faixas de deslocamentos químicos caracterizem o esqueleto drimano no qual o carbono 8 seja um carbono quaternário de dupla. Para a determinação de outros sinais que caracterizem a posição da dupla, outras pesquisas foram realizadas com os carbonos $4,7,8,10$ e 4, 8, 10,12, obtendo-se um percentual de reconhecimento de $94 \%$ para o subesqueleto 7 en-drimano e de $100 \%$ para o $8(12)$ en-drimano. 
O procedimento descrito para o esqueleto drimano foi realizado para vários esqueletos e as faixas de deslocamentos químicos obtidas estão apresentadas na tabela 15, no item resultados. Para a obtenção do percentual de reconhecimento dos esqueletos ou subesqueletos, levou-se em consideração o padrão de carbonos dos mesmos. Por exemplo, no caso acima discutido, em que as faixas obtidas para o esqueleto drimano também são observadas em alguns longipinanos, poderiamos dizer que o percentual de acerto foi de $100 \%$, já que estes esqueletos podem ser diferenciados pelos números de carbonos metinicos e metílicos.

\subsection{7- O sistema SESQREG}

Este sistema consiste na reunião dos vários programas desenvolvidos para determinação de esqueletos, utilizando também as regras heurísticas (faixas de deslocamentos químicos característicos de subesqueletos) obtidas através do sistema PICKUP, bem como o padrão dos tipos de carbonos de cada esqueleto (tabela 12). Os resultados obtidos por estas técnicas são reduzidos a um único número que representa a probabilidade global da substância questionada apresentar um determinado tipo de esqueleto.

O SESQREG é uma adaptação do programa LACSREG ${ }^{28}$, usado na análise de sesquiterpenos lactonizados, para a classe de sesquiterpenos não-lactonizados, tendo sido realizadas as seguintes modificações.

1. Inclusão da opção que permite a criação de um banco de regras heurísticas para sesquiterpenos. Estas regras não foram utilizadas na criação do programa LACSREG.

2. Acréscimo de mais quatro faixas de deslocamentos químicos para desfuncionalização de esqueletos (tabela 11).

3. Alteração do cálculo da probabilidade, que passa a ser uma média ponderada das probabilidades fornecidas pelos programas especificos.

O menu prinicipal do programa apresenta 9 opções ao usuário: 
1. Montar banco de dados

2. Entrar com dados de $\mathrm{RMN}{ }^{13} \mathrm{C}$

3. Executar Regras

4. Executar SISCONST

5. Executar $\mathrm{C} 13 \mathrm{MACH}$

6. Executar SISOCBOT

7. Executar MASMACH

8. Executar $\mathrm{H} 1 \mathrm{MACH}$

9. Combinar resultados

A opção 1 permite a entrada das regras de $\mathrm{RMN}{ }^{13} \mathrm{C}$ obtidas para cada subesqueleto (Tabela 15), juntamente com os padrões dos tipos de carbonos de cada esqueleto presente no banco de dados. Este padrão consiste no número de carbonos quaternários, metínicos, metilênicos e metílicos e no número total de carbonos de cada esqueleto (Tabela 12).

Após a entrada dos dados de $\mathrm{RMN}{ }^{13} \mathrm{C}$ (deslocamentos químicos e multiplicidades), inicia-se a análise com a opção 3, que permite a desfuncionalização do espectro questionado. Esta desfuncionalização é feita com base nas faixas de deslocamentos químicos normalmente observadas para um dado grupo funcional, considerando também a mudança na multiplicidade (Tabela 11). Deste modo, o programa verifica quais os esqueletos do banco que apresentam o mesmo padrão de carbonos do espectro desfuncionalizado, e dentre estes, quais possuem deslocamentos químicos característicos que também façam parte do espectro questionado. O resultado é uma lista dos prováveis esqueletos com as respectivas probabilidades. 
Tabela 11. Dados utilizados para desfuncionalização do espectro questionado.

\begin{tabular}{|c|c|c|c|}
\hline $\begin{array}{l}\text { FUNÇÄO } \\
\text { QUÍMICA }\end{array}$ & $\begin{array}{l}\text { MULTIPLICIDADE } \\
\text { INICIAL }\end{array}$ & $\begin{array}{c}\text { MULTIPILICIDADE } \\
\text { FINAL }\end{array}$ & $\begin{array}{c}\text { FAIXA DE } \\
\text { DESLOCAMENTO } \\
\text { QUIMICO }\end{array}$ \\
\hline$C=0$ & 1 & 3 & 190 a 250 \\
\hline $\mathrm{CHO}$ & 2 & 4 & \\
\hline R-COO-R' & 1 & 4 & 167 a 189 \\
\hline \multirow{3}{*}{$C=C$} & 1 & 2 & \multirow[t]{2}{*}{107 a 166} \\
\hline & 2 & 3 & \\
\hline & 3 & 4 & 100 a 166 \\
\hline \multirow{3}{*}{$\mathrm{C}-\mathrm{OH}$} & 1 & 2 & \multirow{3}{*}{58 a 90} \\
\hline & 2 & 3 & \\
\hline & 3 & 4 & \\
\hline $\mathrm{C}(\mathrm{OR})_{2}$ & 1 & 3 & 104 a 106 \\
\hline $\mathrm{CH}(\mathrm{OR})_{2}$ & 2 & 4 & 91 a 102 \\
\hline
\end{tabular}

As opções 4 e 5 dão acesso aos programas SISCOSNT e C13MACH, que também requerem dados de $\mathrm{RMN}{ }^{13} \mathrm{C}$, porém utilizam diferentes técnicas de confrontação e já foram discutidos anteriormente. Os programas que utilizam dados de RMN ${ }^{1} \mathrm{H}$ e EM (opções 7 e 8 ) não foram utilizados neste trabalho, tendo em vista que a análise destes dados não fazia parte do nosso objetivo.

O programa SISOCBOT fornece os esqueletos mais prováveis baseado nos tipos de esqueletos de maior ocorrência na família e/ou gênero do qual a substância desconhecida foi isolada.

A probabilidade global é a média ponderada das probabilidades fornecidas pelos programas especificos, sendo atribuidos os seguintes pesos aos programas SISCONST, C13MACH, SISOCBOT e REGRAS, respectivamente: 0,8;0,6;0,2 e 0,9, de acordo com o percentual de acerto de cada programa nos 60 testes realizados. 
Tabela 12. Relação dos números de carbonos quaternários, metínicos, metilênicos, metílicos e o número total de carbonos dos esqueletos de sesquiterpenos presentes no banco de dados.

\begin{tabular}{|c|c|c|c|c|c|}
\hline ESQUELETO & No. C & $\mathrm{C}$ & $\mathrm{CH}$ & $\mathrm{CH}_{2}$ & $\mathrm{CH}_{3}$ \\
\hline $11,12,14,15-T E T R A-N O R-P T E R O S I N$ & 11 & 0 & 4 & 5 & 2 \\
\hline $11,12,14,15-T E T R A-N O R-P T E R O S I N$ & 11 & 0 & 4 & 5 & 2 \\
\hline 11,13,14,15-TETRA-NOR-PTEROSIN & 11 & 0 & 4 & 5 & 2 \\
\hline $11,12,13,15$-TETRANOR-EREMOFILANO & 11 & 1 & 1 & 8 & 1 \\
\hline ESQ56 & 12 & 0 & 2 & 6 & 4 \\
\hline 11,12,13-TRINOR-FARNESANO & 12 & 0 & 2 & 6 & 4 \\
\hline 11,12,13-TRINOR-BISABOLANO & 12 & 0 & 3 & 6 & 3 \\
\hline 11,13,14-TRINOR-PTEROSIN & 12 & 0 & 5 & 4 & 3 \\
\hline 11,13,14-TRINOR-12(->4)-PTEROSIN & 12 & 0 & 5 & 4 & 3 \\
\hline 11,14,15-TRINOR-PTEROSIN & 12 & 0 & 5 & 4 & 3 \\
\hline 11,12,14-TRINOR-OXEPINA & 12 & 1 & 1 & 7 & 3 \\
\hline 11,12,13-TRINOR-ELEMANO & 12 & 1 & 2 & 5 & 4 \\
\hline 11,12,13-TRINOR-EREMOFILANO & 12 & 1 & 2 & 7 & 2 \\
\hline $11,12,13-T R I N O R-V E T I S P I R A N O$ & 12 & 1 & 2 & 7 & 2 \\
\hline 11,12,13-TRINOR-EUDESMANO & 12 & 1 & 2 & 7 & 2 \\
\hline 12,13,15-TRINOR-HIRSUTANO & 12 & 1 & 3 & 7 & 1 \\
\hline 13,14,15-TRINOR-ISHWARANO & 12 & 1 & 4 & 7 & 0 \\
\hline 10,11,15-TRINOR-PINGUISANO & 12 & 2 & 1 & 6 & 3 \\
\hline 1,2-DINOR-FARNESANO & 13 & 0 & 2 & 7 & 4 \\
\hline ESQ58 & 13 & 0 & 3 & 7 & 3 \\
\hline ESQ24 & 13 & 0 & 3 & 7 & 3 \\
\hline 13,14-DINOR-DRIMANO & 13 & 0 & 5 & 5 & 3 \\
\hline 10,11-DINOR-MONOCICLOFARNESANO & 13 & 1 & 2 & 6 & 4 \\
\hline 12,13-DINOR-ELEMANO & 13 & 1 & 3 & 4 & 5 \\
\hline 14,15-DINOR-13(->8)-ISHWARANO & 13 & 2 & 3 & 7 & 1 \\
\hline ESQ33 & 14 & 0 & 3 & 8 & 3 \\
\hline 13-NOR-BISABOLANO & 14 & 0 & 3 & 8 & 3 \\
\hline ESQ3 & 14 & 0 & 4 & 4 & 6 \\
\hline ESQ5 & 14 & 0 & 4 & 6 & 4 \\
\hline ESQ57 & 14 & 0 & 4 & 6 & 4 \\
\hline 14-NOR-1,2-SECO-CADINANO & 14 & 0 & 4 & 6 & 4 \\
\hline 14-NOR-EREMOFILANO & 14 & 0 & 5 & 6 & 3 \\
\hline 14-NOR-GUAIANO & 14 & 0 & 5 & 6 & 3 \\
\hline 14-NOR-CADINANO & 14 & 0 & 5 & 6 & 3 \\
\hline 12-NOR-CARIOFILANO & 14 & 0 & 5 & 6 & 3 \\
\hline 15-NOR-EUDESMANO & 14 & 0 & 5 & 6 & 3 \\
\hline 11-NOR-PTEROSIN & 14 & 0 & 6 & 4 & 4 \\
\hline 11-NOR-MONOCICLOFARNESANO & 14 & 1 & 3 & 5 & 5 \\
\hline 2,3-SECO-3-NOR-EREMOFILANO & 14 & 1 & 3 & 5 & 5 \\
\hline 13-NOR-ELEMANO & 14 & 1 & 3 & 5 & 5 \\
\hline 14-NOR-CARIOFILANO & 14 & 1 & 3 & 7 & 3 \\
\hline 15-NOR-SESQUICARANO & 14 & 1 & 3 & 7 & 3 \\
\hline 14-NOR-EUDESMANO & 14 & 1 & 3 & 7 & 3 \\
\hline 13-NOR-NARDOSINANO & 14 & 1 & 3 & 7 & 3 \\
\hline 13-NOR-EREMOFILANO & 14 & 1 & 3 & 7 & 3 \\
\hline ESQ59 & 14 & 1 & 4 & 5 & 4 \\
\hline 11-NOR-14(->3)-DRIMANO & 14 & 1 & 4 & 5 & 4 \\
\hline 15-NOR-BOURBONANO & 14 & 1 & 5 & 5 & 3 \\
\hline
\end{tabular}


Tabela 12. Continuação

\begin{tabular}{|c|c|c|c|c|c|}
\hline ESQUELETO & No. C & $\mathrm{C}$ & $\mathrm{CH}$ & $\mathrm{CH}_{2}$ & $\mathrm{CH}_{3}$ \\
\hline 11-NOR-DRIMANO & 14 & 2 & 2 & 6 & 4 \\
\hline 11-NOR-12(->7)-DRIMANO & 14 & 2 & 2 & 6 & 4 \\
\hline 15-NOR-PINGUISANO & 14 & 2 & 2 & 6 & 4 \\
\hline 12-NOR-DRIMANO & 14 & 2 & 2 & 6 & 4 \\
\hline ESQ62 & 14 & 2 & 3 & 4 & 5 \\
\hline 13-NOR-SILFIPERFOLANO & 14 & 2 & 3 & 6 & 3 \\
\hline 1-NOR-PROTOILUDANO & 14 & 2 & 3 & 6 & 3 \\
\hline 12-NOR-GIMNOMITRANO & 14 & 3 & 1 & 7 & 3 \\
\hline 15-NOR-TUJOPSANO & 14 & 3 & 1 & 7 & 4 \\
\hline 14-NOR-ISHWARANO & 14 & 3 & 2 & 7 & 2 \\
\hline FARNESANO & 15 & 0 & 3 & 7 & 5 \\
\hline 1,2-SECO-CADINANO & 15 & 0 & 4 & 6 & 5 \\
\hline ESQ6 & 15 & 0 & 4 & 7 & 4 \\
\hline BISABOLANO & 15 & 0 & 4 & 7 & 4 \\
\hline 4,5-SECO-EREMOFILANO & 15 & 0 & 4 & 7 & 4 \\
\hline 14(->6)-5,6-SECO-EREMOFILANO & 15 & 0 & 4 & 7 & 4 \\
\hline 4,5-SECO-GUAIANO & 15 & 0 & 4 & 7 & 4 \\
\hline ESQ9 & 15 & 0 & 4 & 7 & 4 \\
\hline GERMACRANO & 15 & 0 & 4 & 7 & 4 \\
\hline 10-SECO-EUDESMANO & 15 & 0 & 5 & 5 & 5 \\
\hline 4,5-SECO-CADINANO & 15 & 0 & 5 & 5 & 5 \\
\hline ESQ29 & 15 & 0 & 5 & 5 & 5 \\
\hline 13(->2)-MONOCICLOFARNESANO & 15 & 0 & 5 & 5 & 5 \\
\hline 15(->1)-4,5-SECO-EREMOFILANO & 15 & 0 & 5 & 5 & 5 \\
\hline ESQ46 & 15 & 0 & 5 & 5 & 5 \\
\hline 1,10-SECO-CADINANO & 15 & 0 & 5 & 6 & 4 \\
\hline ESQ19 & 15 & 0 & 5 & 7 & 3 \\
\hline ESPINIFERIN-2 & 15 & 0 & 5 & 7 & 3 \\
\hline ESQ26 & 15 & 0 & 5 & 7 & 3 \\
\hline OPLOPANANO & 15 & 0 & 6 & 5 & 4 \\
\hline ISOCAROTANO & 15 & 0 & 6 & 5 & 4 \\
\hline VALERENANO & 15 & 0 & 6 & 5 & 4 \\
\hline ESQ18 & 15 & 0 & 6 & 5 & 4 \\
\hline 13(->10)-VALERENANO & 15 & 0 & 6 & 5 & 4 \\
\hline ESQ25 & 15 & 0 & 6 & 5 & 4 \\
\hline 14(->6)-EREMOFILANO & 15 & 0 & 6 & 5 & 4 \\
\hline CADINANO & 15 & 0 & 6 & 5 & 4 \\
\hline 15(->1)-EUDESMANO & 15 & 0 & 6 & 5 & 4 \\
\hline PACIFIGORGIANO & 15 & 0 & 6 & 5 & 4 \\
\hline GUAIANO & 15 & 0 & 6 & 5 & 4 \\
\hline 1-3-EUDESMANO & 15 & 0 & 7 & 4 & 4 \\
\hline 6-8-GUAIANO & 15 & 0 & 8 & 3 & 4 \\
\hline HUMULANO & 15 & 1 & 2 & 8 & 4 \\
\hline 4,5-SECO-EUDESMANO & 15 & 1 & 2 & 8 & 4 \\
\hline 10,11-SECO-HIMACHALANO & 15 & 1 & 2 & 8 & 4 \\
\hline 6,10-SECO-CAROTANO & 15 & 1 & 2 & 8 & 4 \\
\hline 8,9-SECO-LACTARANO & 15 & 1 & 3 & 5 & 6 \\
\hline MONOCICLOFARNESANO & 15 & 1 & 3 & 6 & 5 \\
\hline 4,5-SECO-CARIOFILANO & 15 & 1 & 3 & 6 & 5 \\
\hline 13(->6)-MONOCICLOFARNESANO & 15 & 1 & 3 & 6 & 5 \\
\hline OXEPINE & 15 & 1 & 3 & 6 & 5 \\
\hline
\end{tabular}


Tabela 12. Continuação

\begin{tabular}{|c|c|c|c|c|c|}
\hline ESQUELETO & No. C & $\mathrm{C}$ & $\mathrm{CH}$ & $\mathrm{CH}_{2}$ & $\mathrm{CH}_{3}$ \\
\hline $\begin{array}{l}\text { ESQ15 } \\
9,10-S E C O-D R I M A N O\end{array}$ & $\begin{array}{l}15 \\
15\end{array}$ & $\begin{array}{l}1 \\
1\end{array}$ & $\begin{array}{l}3 \\
3\end{array}$ & $\begin{array}{l}6 \\
6\end{array}$ & $\begin{array}{l}5 \\
5\end{array}$ \\
\hline ESQ47 & 15 & 1 & 3 & 7 & 4 \\
\hline ESQ23 & 15 & 1 & 3 & 7 & 4 \\
\hline ESQ10 & 15 & 1 & 3 & 8 & 3 \\
\hline NEOLEMNANO & 15 & 1 & 3 & 8 & 3 \\
\hline ESPINIFERIN-1 & 15 & 1 & 3 & 8 & 3 \\
\hline 3,4-SECO-13(->9)-DRIMANO & 15 & 1 & 4 & 4 & 6 \\
\hline ELEMANO & 15 & 1 & 4 & 4 & 6 \\
\hline FITUBERIN & 15 & 1 & 4 & 4 & 6 \\
\hline EREMOFILANO & 15 & 1 & 4 & 6 & 4 \\
\hline ESQ12 & 15 & 1 & 4 & 6 & 4 \\
\hline LAURANO & 15 & 1 & 4 & 6 & 4 \\
\hline FURODISANO & 15 & 1 & 4 & 6 & 4 \\
\hline 14(->4)-CAROTANO & 15 & 1 & 4 & 6 & 4 \\
\hline ESQ7 & 15 & 1 & 4 & 6 & 4 \\
\hline 12(->4)-CHAMIGRANO & 15 & 1 & 4 & 6 & 4 \\
\hline PSEUDOGUAIANO & 15 & 1 & 4 & 6 & 4 \\
\hline BULATANO & 15 & 1 & 4 & 6 & 4 \\
\hline GORGONANO & 15 & 1 & 4 & 6 & 4 \\
\hline LEPIDOZANO & 15 & 1 & 4 & 6 & 4 \\
\hline 1,10-SECO-AROMADENDRANO & 15 & 1 & 4 & 6 & 4 \\
\hline EUDESMANO & 15 & 1 & 4 & 6 & 4 \\
\hline NARDOSINANO & 15 & 1 & 4 & 6 & 4 \\
\hline ASTERISCANO & 15 & 1 & 4 & 6 & 4 \\
\hline ESQ14 & 15 & 1 & 4 & 6 & 4 \\
\hline ACORANO & 15 & 1 & 4 & 6 & 4 \\
\hline CARIOFILANO & 15 & 1 & 4 & 6 & 4 \\
\hline CAROTANO & 15 & 1 & 4 & 6 & 4 \\
\hline VETISPIRANO & 15 & 1 & 4 & 6 & 4 \\
\hline BERGAMOTANO & 15 & 1 & 4 & 6 & 4 \\
\hline HIDRINDANO & 15 & 1 & 4 & 6 & 4 \\
\hline ESQ11 & 15 & 1 & 4 & 6 & 4 \\
\hline 5-10,4,5-SECO-GUAIANO & 15 & 1 & 4 & 6 & 4 \\
\hline AMBIGUANO & 15 & 1 & 4 & 6 & 4 \\
\hline HIMACHALANO & 15 & 1 & 4 & 6 & 4 \\
\hline IPIONANO & 15 & 1 & 4 & 6 & 4 \\
\hline BICICLOGERMACRANO & 15 & 1 & 4 & 6 & 4 \\
\hline$\beta$-SANTALANO & 15 & 1 & 4 & 6 & 4 \\
\hline CHILOSCIFANO & 15 & 1 & 4 & 6 & 4 \\
\hline ESQ53 & 15 & 1 & 4 & 6 & 4 \\
\hline ESQ4 & 15 & 1 & 4 & 6 & 4 \\
\hline 11-13-LAURANO & 15 & 1 & 4 & 7 & 3 \\
\hline 14-15-CARIOFILANO & 15 & 1 & 4 & 8 & 2 \\
\hline 12-13-EREMOFILANO & 15 & 1 & 4 & 8 & 2 \\
\hline 5(->4)-LACTARANO & 15 & 1 & 5 & 4 & 5 \\
\hline ESQ31 & 15 & 1 & 5 & 4 & 5 \\
\hline 14(->3)-DRIMANO & 15 & 1 & 5 & 4 & 5 \\
\hline BRASILANO & 15 & 1 & 5 & 4 & 5 \\
\hline
\end{tabular}


Tabela 12. Continuação

\begin{tabular}{|c|c|c|c|c|c|}
\hline ESQUELETO & No. C & $\mathrm{C}$ & $\mathrm{CH}$ & $\mathrm{CH}_{2}$ & $\mathrm{CH}_{3}$ \\
\hline PTEROSIN & 15 & 1 & 5 & 4 & 5 \\
\hline ILUDALANO & 15 & 1 & 5 & 4 & 5 \\
\hline ALIACANO & 15 & 1 & 5 & 4 & 5 \\
\hline TREMULANO & 15 & 1 & 5 & 4 & 5 \\
\hline LACTARANO & 15 & 1 & 5 & 4 & 5 \\
\hline 5-14-CARIOFILANO & 15 & 1 & 5 & 6 & 3 \\
\hline ROTUNDANO & 15 & 1 & 5 & 6 & 3 \\
\hline 2,3-SECO-AROMADENDRANO & 15 & 1 & 6 & 2 & 6 \\
\hline EPICUBEBOL & 15 & 1 & 6 & 4 & 4 \\
\hline AROMADENDRANO & 15 & 1 & 6 & 4 & 4 \\
\hline COPAANO & 15 & 1 & 6 & 4 & 4 \\
\hline CUBEBANO & 15 & 1 & 6 & 4 & 4 \\
\hline BOURBONANO & 15 & 1 & 6 & 4 & 4 \\
\hline ISOCAPNELANO & 15 & 1 & 6 & 4 & 4 \\
\hline 13(->7)-ELEMANO & 15 & 2 & 2 & 5 & 6 \\
\hline TRICOTECANO & 15 & 2 & 2 & 7 & 4 \\
\hline ESQ13 & 15 & 2 & 2 & 7 & 4 \\
\hline ISOCHAMIGRANO & 15 & 2 & 2 & 7 & 4 \\
\hline ISOIPIONANO & 15 & 2 & 2 & 7 & 4 \\
\hline 14(->6)-CUPARANO & 15 & 2 & 2 & 7 & 4 \\
\hline BICICLOHUMULANO & 15 & 2 & 2 & 7 & 4 \\
\hline CUPARANO & 15 & 2 & 2 & 7 & 4 \\
\hline CHAMIGRANO & 15 & 2 & 2 & 7 & 4 \\
\hline TRIFARANO & 15 & 2 & 3 & 5 & 5 \\
\hline 13(->9)-DRIMANO & 15 & 2 & 3 & 5 & 5 \\
\hline 2,3-SECO-LONGIBORNANO & 15 & 2 & 3 & 5 & 5 \\
\hline 3,4-SECO-AFRICANO & 15 & 2 & 3 & 5 & 5 \\
\hline DRIMANO & 15 & 2 & 3 & 5 & 5 \\
\hline PINGUISANO & 15 & 2 & 3 & 5 & 5 \\
\hline FOMANOSIN & 15 & 2 & 3 & 5 & 5 \\
\hline ESQ1 & 15 & 2 & 3 & 5 & 5 \\
\hline 4-14-CARIOFILANO & 15 & 2 & 3 & 7 & 3 \\
\hline 7,11-SECO-ISHWARANO & 15 & 2 & 3 & 7 & 3 \\
\hline OMFALANO & 15 & 2 & 3 & 7 & 3 \\
\hline 4,12-CICLO-TRICOTECANO & 15 & 2 & 3 & 7 & 3 \\
\hline 8,11-SECO-ISHWARANO & 15 & 2 & 3 & 7 & 3 \\
\hline BOTRIANO & 15 & 2 & 4 & 3 & 6 \\
\hline ESQ8 & 15 & 2 & 4 & 3 & 6 \\
\hline ISOCEDRANO & 15 & 2 & 4 & 5 & 4 \\
\hline CIPERANO & 15 & 2 & 4 & 5 & 4 \\
\hline 14(->10)-ARISTOLANO & 15 & 2 & 4 & 5 & 4 \\
\hline HIRSUTANO & 15 & 2 & 4 & 5 & 4 \\
\hline PRESILFIPERFOLANO & 15 & 2 & 4 & 5 & 4 \\
\hline$\alpha$-SANTALANO & 15 & 2 & 4 & 5 & 4 \\
\hline ESQ17 & 15 & 2 & 4 & 5 & 4 \\
\hline ESTERPURANO & 15 & 2 & 4 & 5 & 4 \\
\hline 2-9-CARIOFILANO & 15 & 2 & 4 & 5 & 4 \\
\hline 5(4->10)-ABEOAROMADENDRANO & 15 & 2 & 4 & 5 & 4 \\
\hline PROTOILUDANO & 15 & 2 & 4 & 5 & 4 \\
\hline
\end{tabular}


Tabela 12. Continuação

\begin{tabular}{|c|c|c|c|c|c|}
\hline ESQUELETO & No. C & $\mathrm{C}$ & $\mathrm{CH}$ & $\mathrm{CH}_{2}$ & $\mathrm{CH}_{3}$ \\
\hline CAPNELANO & 15 & 2 & 4 & 5 & 4 \\
\hline SILFIPERFOLANO & 15 & 2 & 4 & 5 & 4 \\
\hline ILUDANO & 15 & 2 & 4 & 5 & 4 \\
\hline PRE-SEICHELANO & 15 & 2 & 4 & 5 & 4 \\
\hline SEICHELANO & 15 & 2 & 4 & 5 & 4 \\
\hline ZIZANO & 15 & 2 & 4 & 5 & 4 \\
\hline LIPIFOLIANO & 15 & 2 & 4 & 5 & 4 \\
\hline PREZIZANO & 15 & 2 & 4 & 5 & 4 \\
\hline MALIANO & 15 & 2 & 4 & 5 & 4 \\
\hline CEDRANO & 15 & 2 & 4 & 5 & 4 \\
\hline 15(->4)-LONGIBORNANO & 15 & 2 & 4 & 5 & 4 \\
\hline LONGIPINANO & 15 & 2 & 4 & 5 & 4 \\
\hline 2-10-HIMACHALANO & 15 & 2 & 4 & 5 & 4 \\
\hline ARISTOLANO & 15 & 2 & 4 & 5 & 4 \\
\hline AFRICANO & 15 & 2 & 4 & 5 & 4 \\
\hline PROBOTRIANO & 15 & 2 & 4 & 5 & 4 \\
\hline 1(11->10)-ABEOMARASMANO & 15 & 2 & 5 & 3 & 5 \\
\hline CLOVANO & 15 & 3 & 1 & 7 & 4 \\
\hline ESQ50 & 15 & 3 & 1 & 8 & 3 \\
\hline THAPSANO & 15 & 3 & 2 & 4 & 6 \\
\hline GIMNOMITRANO & 15 & 3 & 2 & 6 & 4 \\
\hline TUJOPSANO & 15 & 3 & 2 & 6 & 4 \\
\hline SILFINANO & 15 & 3 & 2 & 6 & 4 \\
\hline ISOCOMANO & 15 & 3 & 2 & 6 & 4 \\
\hline 15(->9)-MODEFANO & 15 & 3 & 2 & 6 & 4 \\
\hline ESQ48 & 15 & 3 & 2 & 6 & 4 \\
\hline MILTAILANO & 15 & 3 & 2 & 6 & 4 \\
\hline PUNCTATANO & 15 & 3 & 2 & 6 & 4 \\
\hline MODEFANO & 15 & 3 & 2 & 6 & 4 \\
\hline LONGIBORNANO & 15 & 3 & 2 & 6 & 4 \\
\hline ISOLACTARANO & 15 & 3 & 3 & 4 & 5 \\
\hline MARASMANO & 15 & 3 & 3 & 4 & 5 \\
\hline SOLANASCONO & 15 & 3 & 3 & 6 & 3 \\
\hline ISHWARANO & 15 & 3 & 3 & 6 & 3 \\
\hline LONGICICLANO & 15 & 3 & 4 & 4 & 4 \\
\hline CICLOMILTAILANO & 15 & 4 & 2 & 5 & 4 \\
\hline 2-Me-MONOCICLOFARNESANO & 16 & 1 & 4 & 5 & 6 \\
\hline ESQ38 & 16 & 2 & 3 & 6 & 5 \\
\hline ESQ54 & 18 & 0 & 6 & 10 & 2 \\
\hline ESQ36 & 21 & 1 & 4 & 12 & 4 \\
\hline ESQ27 & 21 & 1 & 4 & 12 & 4 \\
\hline ESQ28 & 21 & 1 & 4 & 12 & 4 \\
\hline ESQ20 & 21 & 2 & 4 & 11 & 4 \\
\hline ESQ22 & 21 & 2 & 4 & 11 & 4 \\
\hline ESQ21 & 21 & 2 & 4 & 11 & 4 \\
\hline DRI-8O,15-CM & 21 & 2 & 4 & 11 & 4 \\
\hline ESQ42 & 22 & 0 & 5 & 12 & 5 \\
\hline ESQ44 & 22 & 0 & 6 & 12 & 4 \\
\hline ESQ32 & 22 & 1 & 5 & 11 & 5 \\
\hline EUD-3,4O-CM & 22 & 1 & 6 & 11 & 4 \\
\hline
\end{tabular}


Tabela 12. Continuação

\begin{tabular}{lccccc}
\hline \multicolumn{1}{c}{ ESQUELETO } & No. C & $\mathrm{C}$ & $\mathrm{CH}$ & $\mathrm{CH}_{2}$ & $\mathrm{CH}_{3}$ \\
\hline \hline AROMAD-14-CM1 & & & & & \\
ESQ52 & 22 & 1 & 7 & 11 & 3 \\
15-ME-DRI-8O,15-CM & 22 & 2 & 5 & 10 & 5 \\
ESQ41 & 22 & 2 & 5 & 10 & 5 \\
ESQ43 & 22 & 2 & 5 & 10 & 5 \\
ESQ51A & 23 & 0 & 6 & 11 & 6 \\
ESQ51B & 23 & 2 & 4 & 13 & 4 \\
ESQ37 & 23 & 2 & 5 & 12 & 4 \\
ESQ60 & 24 & 0 & 5 & 14 & 5 \\
ESQ55 & 24 & 0 & 8 & 10 & 6 \\
ESQ35 & 25 & 1 & 10 & 8 & 6 \\
2-2'-BIS-BISABOLANO & 28 & 2 & 8 & 10 & 8 \\
12-14'-BIS-14(->6)-8,12-FURANOEREMOFILANO & 30 & 0 & 10 & 12 & 8 \\
ESQ45 & 30 & 0 & 12 & 12 & 6 \\
2-BIS-CADINANO & 30 & 0 & 13 & 10 & 7 \\
6-8',15-9'-BIS-LINDENANO & 30 & 0 & 14 & 8 & 8 \\
15-15'-BIS-ISOCUPARANO & 30 & 2 & 15 & 6 & 7 \\
3-15'-BIS-ISOCUPARANO & 30 & 4 & 4 & 16 & 6 \\
ESQ40 & 30 & 4 & 5 & 14 & 7 \\
\hline
\end{tabular}




\subsection{8- O programa MACRONO ${ }^{28,38}$}

Em geral, os produtos naturais apresentam grupos substituintes (angelato, butirato, etc...), ligados ao esqueleto carbônico por meio de um heteroátomo, sendo normalmente provenientes da esterificação de hidroxilas do esqueleto da substância com ácidos carboxílicos. No SISTEMAT, estes grupos são denominados macronós.

Durante a análise espectral, a presença dos sinais pertencentes aos macronós pode confundir os algoritmos de identificação, conduzindo a propostas estruturais incorretas, ou até mesmo, resultar numa explosão combinatória durante a geração de estruturas. Para resolver este problema, foi desenvolvido o programa MACRONO, que fornece os possíveis grupos ligados ao esqueleto carbônico por meio de heteroátomos. Deste modo, é feita uma análise prévia do espectro da substânciaproblema, antes que ele seja submetido aos programas que permitem a identificação de esqueleto e as propostas estruturais.

Foi criado, então, um banco de dados com os sinais de $\mathrm{RMN}{ }^{13} \mathrm{C}$ dos substituintes mais comuns encontrados em terpenóides, utilizando-se os dados do levantamento bibliográfico realizado.

Os dados de $\mathrm{RMN}{ }^{13} \mathrm{C}$ da substância-problema são fornecidos ao programa e correlacionados com as informaçōes contidas no banco de dados, de modo a se determinar a presença de um macronó e quais os sinais do espectro da amostra que podem ser atribuídos a ele.

Este aplicativo foi testado com os dados de RMN ${ }^{13} \mathrm{C}$ do sesquiterpeno da figura 19, que não constava do banco de dados, sendo fornecidos pelo programa os resultados apresentados na tabela 13. Estes dados permitem propor a existência dos grupos acetato, furoato e 2-metil-butirato. No entanto, como ainda restaram cinco sinais, além dos quinze que correspodem ao esqueleto sesquiterpênico, os dados de $\mathrm{RMN}{ }^{13} \mathrm{C}$ foram submetidos novamente ao programa MACRONO, tendo sido retirados os sinais já atribuídos aos grupos supramencionados. Dentre os macronós apresentados, o único com cinco sinais e menor erro médio foi o 2-metil-butirato (tabela 14). 


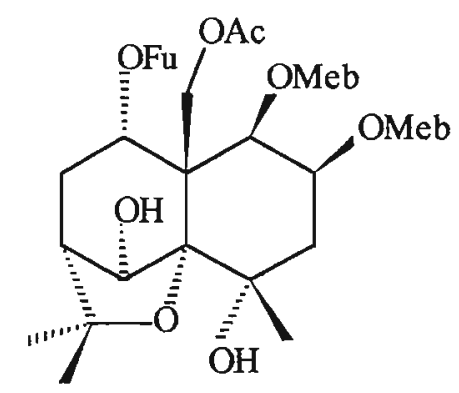

Espectro de RMN ${ }^{13} \mathrm{C}: 69.7(\mathrm{~d}), 67.6(\mathrm{~d}), 41,2(\mathrm{t}), 72.2(\mathrm{~s}), 91.1(\mathrm{~s}), 78.7(\mathrm{~d}), \quad 50.2(\mathrm{~d}), 34.4(\mathrm{t})$, 68.8(d), 53.6(s), 84.8(s), 26.4(q), 30.0(q), 24.6(q), 65.8(d), 21.4(q), 170.3(s), 109.9(d), 118.7(s), $143.7(\mathrm{~d}), 148.9(\mathrm{~d}), 161.8(\mathrm{~s}), 11.3(\mathrm{q}), 11.6(\mathrm{q}), 15.3(\mathrm{q}), 16.4(\mathrm{q}), 25.5(\mathrm{t}), 26.5(\mathrm{t}), 40.6(\mathrm{~d}), 41.6(\mathrm{~d})$, 174.5(s), 175.1(s).

Figura 19. Dados utilizados para testar o programa MACRONO.

Tabela 13. Resultados dos testes com o programa MACRONO.

\begin{tabular}{|c|l|c|c|}
\hline MACRONO & \multicolumn{1}{|c|}{ SINAIS } & ERRO MÉDIO & No. C \\
\hline Acetato & $170.3,21.4$ & 0.850 & 2 \\
\hline Furoato & $161.8,148.9,143.7,118.7,109.9$ & 1.820 & 5 \\
\hline 2-Me-Butirato & $175.1,41.6,26.5,16.4,11.6$ & 0.820 & 5 \\
\hline Propionato & $175.1,26.5,11.3$ & 2.167 & 3 \\
\hline
\end{tabular}

Tabela 14. Resultados dos testes com o programa MACRONO, após a retirada dos sinais dos grupos acetato, furoato e 2-metil-butirato.

\begin{tabular}{|l|l|c|c|}
\hline \multicolumn{1}{|c|}{ MACRONÓ } & \multicolumn{1}{|c|}{ SINAIS } & ERRO MÉDIO & No. C \\
\hline 2-Me-butirato & $174.5,40.6,25.5,15.3,11.3$ & 1.380 & 5 \\
\hline Isopropionato & $65.8,26.4,24.6$ & 1.733 & 3 \\
\hline 2,3-ep-n-but & $174.5,65.8,50.2,15.3$ & 1.875 & 4 \\
\hline propionato & $174.5,25.5,11.3$ & 2.700 & 3 \\
\hline
\end{tabular}

Deste modo, os sinais referentes a um grupo furoato, um acetato e dois grupos 2-Me-butirato podem ser retirados do espectro, restando apenas os 15 sinais que correspodem ao esqueleto carbônico do sesquiterpeno. 


\section{RESULTADOS}

Para se avaliar a eficiência dos programas aplicativos do SISTEMAT no processo de determinação estrutural, foram utilizados os dados referentes a 60 sesquiterpenos, publicados na revista Phytochemistry, no período de janeiro de 1996 a janeiro de 1998, sendo considerados apenas aqueles ditos inéditos ou que ainda não estão presentes no banco de dados. Os resultados obtidos para cada uma das substâncias são apresentados nas páginas a seguir, bem como as faixas de deslocamentos químicos obtidos através do sistema PICKUP (tabela 15).

Nas tabelas de probabilidade de esqueleto são apresentados somente os oito primeiros esqueletos mais prováveis, portanto a somatória das probabilidades constantes nas tabelas não será igual a $100 \%$. O resultado global é uma média ponderada das probabilidades fornecidas pelos programas específicos, tendo sido atribuidos os pesos 0,$9 ; 0,8 ; 0,6$ e 0,2 aos programas REGRAS, SISCONST, C13MACH e SISOCBOT, respectivamente, de acordo com os percentuais de acerto observados para estes programas nos 60 testes realizados.

Além das probabilidades de esqueleto fornecidas pelos programas supramencionados, também são apresentadas as subestruturas propostas pelo programa SISCONST. AOs carbonos de cada subestrutura estão associados os deslocamentos químicos do espectro em análise, cuja atribuição é feita pela comparação com os deslocamentos químicos das subestruturas compatíveis com o espectro questionado, obtidas a partir das substâncias presentes no banco de dados.

Nos testes realizados com o programa SISCONST foram solicitados, em geral, um número mínimo de sete átomos para as subestruturas fornecidas e uma faixa de erro de $1,0 \delta$ Nos casos em que o programa não encontra subestruturas com desiocamentos químicos na faixa de erro de $1,0 \delta$, esta é aumentada automaticamente para 2,0 e 3,0 $\delta$. A faixa de erro máxima utilizada nos testes realizados foi de $5,0 \delta$, levando-se em consideração possiveis efeitos de solvente.

Em alguns testes são apresentadas subestruturas com um número de átomos inferior a sete, as quais foram fornecidas pelo SISCONST após a retirada dos sinais atribuídos aos átomos da primeira subestrutura proposta.

O número de subestruturas propostas pelo SISCONST varia com a faixa de erro considerada, com o número mínimo de átomos requerido para as subestruturas e com 0 tipo de substância analisada. A faixa de erro igual a $1,0 \delta$ conduziu a um 
número máximo de 12 subestruturas para os compostos aqui analisados. Já a faixa de 2,0-3,0 $\delta$ resultou num número máximo de 30 subestruturas. Para uma faixa de 5,0 $\delta$ foi obtido em alguns casos um número máximo de $100-150$ subestruturas. Muitas das subestruturas propostas, no entanto, são sobreponíveis ou diferem apenas na estereoquímica de um determinado grupo, ou então, diferem com relação à atribuição dos sinais de $\mathrm{RMN}{ }^{13} \mathrm{C}$. As subestruturas estão representadas da mesma forma que elas aparecem na tela do programa SISCONST, subentendendo-se a presença dos átomos de hidrogênio. Nos testes realizados são apresentadas as principais subestruturas fornecidas pelo programa, ou seja, as maiores ou as subestruturas menores que sejam complementares àquelas de maior tamanho.

Também são apresentados os resultados fornecidos pelo programa MACRONO para as substâncias que apresentam grupos substituintes. Este programa permite a retirada dos sinais que não pertencem ao esqueleto da substância e que poderiam conduzir a uma probabilidade de esqueleto errada ou a propostas incorretas de subestruturas no programa SISCONST. Nestes casos, os dados de RMN ${ }^{13} \mathrm{C}$ da substância são submetidos ao SISCONST apenas após a retirada dos sinais atribuídos pelo programa MACRONO a um determinado grupo substituinte.

No programa $\mathrm{C} 13 \mathrm{MACH}$, a probabilidade de esqueleto foi obtida com base nas dez primeiras substâncias com maior indice de semelhança, dentro de uma faixa de erro de $2,0 \delta$. Estas são as condições que permitem a obtenção de melhores resultados, de acordo com os testes realizados, variando-se a faixa de erro e o número de substâncias requisitadas com maior índice de semelhança

O programa SESQREG indica 0 subesqueleto e o percentual de reconhecimento, a partir da confrontação do espectro em análise com as faixas de deslocamentos químicos obtidas para os diversos subesqueletos. Quando não existem regras para um subesqueleto, o programa fornece os prováveis esqueletos, baseado na comparação entre o número de sinais do espectro de $\mathrm{RMN}{ }^{13} \mathrm{C}$ e 0 número de átomos de carbonos quaternários, metínicos, metilênicos e metílicos, calculado após a desfuncionalização do esqueleto (tabela 12).

Também foi averiguada a possibilidade de utilização dos programas SISCONST e C13MACH para a identificação de sesquiterpenos em misturas, por exemplo, em óleos essenciais. Nos testes realizados foram utilizados os dados espectrais de uma fração de óleo essencial da planta Xylopia emarginata, obtida no Laboratório de Produtos Naturais do IQ-USP, bem como dados espectrais de RMN ${ }^{13} \mathrm{C}$ de óleos essenciais citados na literatura (J. Essential. Oil Res., 1997, 9, 321 e Magn. Res. Chem., 1995, 233). Nos casos onde já são apresentados os espectros dos 
Res. Chem., 1995, 233). Nos casos onde já são apresentados os espectros dos componentes puros, usamos como dados de entrada os deslocamentos químicos de todos os componentes com suas respectivas multiplicidades, combinados aleatoriamente.

Na tabela 15, onde são apresentadas as faixas de deslocamentos químicos característicos de vários tipos de esqueletos ou subesqueletos, observa-se que o número de faixas varia de acordo com o esqueleto ou subesqueleto analisado. Isto deve-se ao fato de alguns esqueletos apresentarem um arranjo carbônico bem característico, como por exemplo, os esqeleto $\alpha$-santalano, ciclomiltailano e 15(->9)modefano, sendo caracterizados facilmente pelos deslocamentos químicos de apenas alguns carbonos. Outros esqueletos mais comuns ou pouco diferenciados requerem um número maior de deslocamentos quimicos para que a sua caracterização se torne possível. 
Tabela 15. Faixas de deslocamentos químicos característicos de subesqueletos de sesquiterpenos e respectivos percentuais de reconhecimento. ${ }^{*}$

\begin{tabular}{|c|c|c|}
\hline Subesqueleto & $\begin{array}{c}\text { Faixas de deslocamentos } \\
\text { químicos (ppm) } \\
\end{array}$ & $\begin{array}{c}\text { Percentual de } \\
\text { Reconhecimento (\%) }\end{array}$ \\
\hline \multirow{6}{*}{$1[1,90 R ; 5,110 \times 1]$} & $80,5-66,8(d, C 1)$ & \multirow{6}{*}{100,0} \\
\hline & $94,5-57,4(s, C 5)$ & \\
\hline & $65,8-43,0(d, C 7)$ & \\
\hline & $80,9-66,0$ (d, c9) & \\
\hline & $56,0-43,9(\mathrm{~s}, \mathrm{C} 10)$ & \\
\hline & $85,8-69,8(\mathrm{~s}, \mathrm{C} 11)$ & \\
\hline \multirow{7}{*}{$1[1,6,90 \mathrm{OR} ; 5,110 \times 1]$} & $80,5-67,8(d, C 1)$ & \multirow{7}{*}{100,0} \\
\hline & $55,5-45,7 \quad(s, C 10)$ & \\
\hline & $80,9-68,0(d, C 9)$ & \\
\hline & $94,5-88,0(s, C 5)$ & \\
\hline & $85,8-69,8 \quad(s, \mathrm{C} 11)$ & \\
\hline & $57,5-48,2(d, C 7)$ & \\
\hline & $80,9-67,9(\mathrm{~d}, \mathrm{C} 6)$ & \\
\hline \multirow{4}{*}{$1[5,110 \times 1 ; 60 \times 0]$} & $86,0-84,9(s, C 5)$ & \multirow{4}{*}{100,0} \\
\hline & $55,5-55,2(d, C 7)$ & \\
\hline & $78,6-77,5(\mathrm{~s}, \mathrm{C} 11)$ & \\
\hline & $212,6-211,0 \quad(s, C 6)$ & \\
\hline \multirow{8}{*}{$1[1,4,9$ OR; $5,110 \mathrm{OI} ; 6 \mathrm{OXO}]$} & $71,0-69,9(\mathrm{~s}, \mathrm{C} 4)$ & \multirow{8}{*}{100,0} \\
\hline & $86,0-84,9(s, C 5)$ & \\
\hline & $212,6-211,0(s, C 6)$ & \\
\hline & $55,5-55,2(d, C 7)$ & \\
\hline & $78,6-77,5(\mathrm{~s}, \mathrm{C} 11)$ & \\
\hline & $72,5-72,0 \quad(d, C 1)$ & \\
\hline & $56,0-55,0(\mathrm{~s}, \mathrm{C} 10)$ & \\
\hline & $72,3-72,0(\mathrm{~d}, \mathrm{C} 9)$ & \\
\hline \multirow{7}{*}{$1[1,4,6,90 \mathrm{OR} ; 5,110 \mathrm{XI}]$} & $76,5-67,8(\mathrm{~s}, \mathrm{C} 1)$ & \multirow{7}{*}{100,0} \\
\hline & $85,1-69,5(\mathrm{~s}, \mathrm{C} 4)$ & \\
\hline & $94,5-91,0(\mathrm{~s}, \mathrm{C5})$ & \\
\hline & $80,4-67,9(\mathrm{~d}, \mathrm{C6})$ & \\
\hline & $65,0-48,2(d, C 7)$ & \\
\hline & $79,6-68,0(d, C 9)$ & \\
\hline & $85,8-69,8(\mathrm{~s}, \mathrm{C} 11)$ & \\
\hline
\end{tabular}

*O número anterior ao colchete indica o número do esqueleto, conforme a figura 9 , o prefixo EP é usado para epóxido. 
Tabela 15. Continuação

\begin{tabular}{|c|c|c|}
\hline Subesqueleto & $\begin{array}{c}\text { Faixas de deslocamentos } \\
\text { químicos (ppm) } \\
\end{array}$ & $\begin{array}{c}\text { Percentual de } \\
\text { Reconhecimento (\%) }\end{array}$ \\
\hline \multirow{5}{*}{$1[7(11) \mathrm{EN} ; 80 \times 0]$} & $41,2-35,4(s, C 10)$ & \multirow{5}{*}{100,0} \\
\hline & $60,0-56,7$ (t, C9) & \\
\hline & $210,6-200,8(s, C 8)$ & \\
\hline & $131,5-129,6(\mathrm{~s}, \mathrm{C} 7)$ & \\
\hline & $146,1-144,1$ (s, C11) & \\
\hline \multirow{4}{*}{$1[80 \times 0]$} & $50,5-44,7$ (d, C5) & \multirow{4}{*}{100,0} \\
\hline & $41,2-32,7(s, C 10)$ & \\
\hline & $60,4-56,7(t, C 9)$ & \\
\hline & $214,3-200,0(\mathrm{~s}, \mathrm{C} 8)$ & \\
\hline \multirow{10}{*}{$1[1,2,3,4,8,9,15 \mathrm{OR} ; 5,11 \mathrm{OXI}]$} & $75,5-70,8(d, C 1)$ & \multirow{10}{*}{100,0} \\
\hline & $73,5-69,0 \quad(s, \mathrm{c} 2)$ & \\
\hline & $78,4-75,1(d, C 3)$ & \\
\hline & $72,6-69,6(s, C 4)$ & \\
\hline & $94,5-93,0(s, C 5)$ & \\
\hline & $52,5-49,2(d, C 7)$ & \\
\hline & $85,5-84,0(s, C 11)$ & \\
\hline & $74,5-68,9(d, C 8)$ & \\
\hline & $76,3-70,5$ (d, C9) & \\
\hline & $62,5-60,2(t, C 15)$ & \\
\hline \multirow{5}{*}{$1[6 \mathrm{EN} ; 80 \times 0]$} & $40,5-36,9(s, C 10)$ & \multirow{5}{*}{100,0} \\
\hline & $58,5-48,2(t, C 9)$ & \\
\hline & $201,6-191,1 \quad(s, C 8)$ & \\
\hline & $147,8-142,3(s, \mathrm{C} 7)$ & \\
\hline & $145,6-133,5(d, C 6)$ & \\
\hline \multirow{5}{*}{$1[7(11) \mathrm{EN}]$} & $41,2-34,2(s, C 10)$ & \multirow{5}{*}{76,9} \\
\hline & $25,7-20,2(q, C 12)$ & \\
\hline & $23,2-18,8(q, C 13)$ & \\
\hline & $131,8-126,9(\mathrm{~s}, \mathrm{C} 7)$ & \\
\hline & $146,1-123,0 \quad(s, C 11)$ & \\
\hline \multirow{4}{*}{$1[1 \mathrm{EN} ; 30 \times 0]$} & $42,4-36,0(s, \mathrm{C} 10)$ & \multirow{4}{*}{100,0} \\
\hline & $160,8-153,1 \quad(d, C 1)$ & \\
\hline & $127,3-125,1(d, C 2)$ & \\
\hline & $204,0-183,6(s, C 3)$ & \\
\hline
\end{tabular}


Tabela 15. Continuação

\begin{tabular}{|c|c|c|}
\hline Subesqueleto & $\begin{array}{l}\text { Faixas de deslocamentos } \\
\text { químicos (ppm) }\end{array}$ & $\begin{array}{c}\text { Percentual de } \\
\text { Reconhecimento (\%) } \\
\end{array}$ \\
\hline \multirow{4}{*}{$1[11 \mathrm{EN} ; 13 \mathrm{OH}]$} & $42,7-41,5$ (d, C7) & \multirow{4}{*}{100,0} \\
\hline & $154,5-135,1(\mathrm{~s}, \mathrm{C} 11)$ & \\
\hline & $108,0-107,1 \quad(t, C 12)$ & \\
\hline & $65,3-64,8(t, C 13)$ & \\
\hline \multirow{6}{*}{$1[4 \mathrm{OR}]$} & $45,2-40,5 \quad(t, C 1)$ & \multirow{6}{*}{55,8} \\
\hline & $39,4-33,7(s, \mathrm{c} 10)$ & \\
\hline & $57,2-46,2(d, C 5)$ & \\
\hline & $83,4-71,6(s, C 4)$ & \\
\hline & $45,4-35,5(t, C 3)$ & \\
\hline & $23,3-19,2(t, C 2)$ & \\
\hline \multirow{9}{*}{$1[5 \mathrm{EN}]$} & $43,5-39,5(t, C 1)$ & \multirow{9}{*}{100,0} \\
\hline & $22,7-17,7 \quad(t, C 2)$ & \\
\hline & $37,7-33,2(t, C 3)$ & \\
\hline & $39,2-33,2(d, C 4)$ & \\
\hline & $27,7-18,7(q, C 14)$ & \\
\hline & $22,3-20,2(q, C 15)$ & \\
\hline & $34,9-34,2$ (s, C10) & \\
\hline & $152,6-133,1 \quad(s, C 5)$ & \\
\hline & $129,1-114,5(\mathrm{~d}, \mathrm{C} 6)$ & \\
\hline \multirow{9}{*}{$1[11 \mathrm{OH}]$} & $44,7-21,7(t, C 9)$ & \multirow{9}{*}{90,6} \\
\hline & $24,7-20,6(t, C 8)$ & \\
\hline & $50,7-41,0(d, c 7)$ & \\
\hline & $40,9-20,5(t, C 6)$ & \\
\hline & $54,9-42,5(d, C 5)$ & \\
\hline & $48,2-30,2(s, C 10)$ & \\
\hline & $29,8-23,5(q, C 12)$ & \\
\hline & $85,0-72,4(s, C 11)$ & \\
\hline & $29,7-22,3(q, C 10)$ & \\
\hline \multirow{6}{*}{$1[5 \mathrm{OH} ; 11 \mathrm{EN}]$} & $41,4-40,5(d, C 4)$ & \multirow{6}{*}{100,0} \\
\hline & $78,5-75,6(\mathrm{~s}, \mathrm{C5})$ & \\
\hline & $150,5-149,3$ (s, C11) & \\
\hline & $108,8-108,1 \quad(t, C 12)$ & \\
\hline & $21,0-20,8(q, C 13)$ & \\
\hline & $20,2-15,5(q, c 14)$ & \\
\hline
\end{tabular}


Tabela 15. Continuação

\begin{tabular}{|c|c|c|}
\hline Subesqueleto & $\begin{array}{l}\text { Faixas de deslocamentos } \\
\text { químicos (ppm) } \\
\end{array}$ & $\begin{array}{c}\text { Percentual de } \\
\text { Reconhecimento (\%) } \\
\end{array}$ \\
\hline \multirow{8}{*}{$1[4(14) E N]$} & $41,9-30,7(t, C 1)$ & \multirow{8}{*}{61,5} \\
\hline & $29,3-22,2(t, C 2)$ & \\
\hline & $41,7-30,2(t, C 3)$ & \\
\hline & $154,1-146,8 \quad(s, C 4)$ & \\
\hline & $112,3-104,9(t, C 14)$ & \\
\hline & $28,210,1 \quad(q, C 15)$ & \\
\hline & $42,2-35,4(s, C 10)$ & \\
\hline & $60,2-43,7(d, c 5)$ & \\
\hline \multirow{6}{*}{$1[1,4 \mathrm{OR}]$} & $91,3-75,3(d, c 1)$ & \multirow{6}{*}{52,8} \\
\hline & $48,5-33,2(s, C 10)$ & \\
\hline & $60,5-44,9(d, c 5)$ & \\
\hline & $82,5-65,9 \quad(s, \mathrm{C} 4)$ & \\
\hline & $45,2-33,0(t, C 3)$ & \\
\hline & $40,7-23,3(t, c 2)$ & \\
\hline \multirow{6}{*}{$1[1 \mathrm{OR}]$} & $83,0-80,3(d, C 1)$ & \multirow{6}{*}{100,0} \\
\hline & $27,2-26,6(t, C 2)$ & \\
\hline & $31,5-27,3(t, C 3)$ & \\
\hline & $33,7-33,0(\mathrm{~s}, \mathrm{C4})$ & \\
\hline & $53,0-45,9(d, C 5)$ & \\
\hline & $40,9-39,2(s, \mathrm{C} 10)$ & \\
\hline \multirow{5}{*}{$1[1 \mathrm{OR} ; 4 \mathrm{EN}]$} & $80,3-75,4(d, C 1)$ & \multirow{5}{*}{50,0} \\
\hline & $27,7-23,8(t, C 2)$ & \\
\hline & $32,7-31,2(t, C 3)$ & \\
\hline & $133,3-123,8(\mathrm{~s}, \mathrm{C} 4)$ & \\
\hline & $133,6-125,4(s, C 5)$ & \\
\hline
\end{tabular}


Tabela 15. Continuação

\begin{tabular}{|c|c|c|}
\hline Subesqueleto & $\begin{array}{c}\text { Faixas de deslocamentos } \\
\text { químicos (ppm) }\end{array}$ & $\begin{array}{c}\text { Percentual de } \\
\text { Reconhecimento (\%) } \\
\end{array}$ \\
\hline & $40,0-28,7(t, c 1)$ & \\
\hline \multirow[t]{6}{*}{$1[3 \mathrm{EN}]$} & $23,7-20,7(t, C 2)$ & 81,8 \\
\hline & $121,8-119,0 \quad(d, C 3)$ & \\
\hline & $137,0-132,6(s, C 4)$ & \\
\hline & $47,4-42,5(d, C 5)$ & \\
\hline & $46,7-30,2(s, C 10)$ & \\
\hline & $82,5-71,0(s, C 4)$ & \\
\hline \multirow{5}{*}{$1[4 \mathrm{OR} ; 6 \mathrm{EN}]$} & $54,4-48,5(d, C 5)$ & 100,0 \\
\hline & $140,8-115,3(d, C 6)$ & \\
\hline & $146,1-142,3(\mathrm{~s}, \mathrm{C} 7)$ & \\
\hline & $39,0-33,7\left(s, C_{10}\right)$ & \\
\hline & $84,5-70,9(\mathrm{~s}, \mathrm{C} 4)$ & \\
\hline \multirow[t]{4}{*}{$1[4 \mathrm{OR} ; 7 \mathrm{EN}]$} & $52,0-42,9(\mathrm{~d}, \mathrm{C5})$ & 100,0 \\
\hline & $33,9-23,1 \quad(t, C 6)$ & \\
\hline & $142,1-141,6(\mathrm{~s}, \mathrm{C} 7)$ & \\
\hline & $116,1-115,8(d, C 8)$ & \\
\hline \multirow{6}{*}{$1[1 \mathrm{OR} ; 3 \mathrm{EN}]$} & $81,0-74,4(d, C 1)$ & \\
\hline & $32,2-29,0 \quad(t, C 2)$ & 100,0 \\
\hline & $125,0118,9(d, C 3)$ & \\
\hline & $153,8-133,5(\mathrm{~s}, \mathrm{C} 4)$ & \\
\hline & $52,2-46,4(d, C 5)$ & \\
\hline & $40,9-36,2(s, C 10)$ & \\
\hline \multirow{5}{*}{$1[5,7(11) \mathrm{EN}]$} & $147,6-142,5(\mathrm{~s}, \mathrm{C5})$ & \\
\hline & $120,5-114,5(d, C 6)$ & 100,0 \\
\hline & $131,8-127,6(s, \mathrm{c})$ & \\
\hline & $128,8-125,5(\mathrm{~s}, \mathrm{C} 10)$ & \\
\hline & $40,2-34,2(s, C 11)$ & \\
\hline \multirow{5}{*}{$1[1,4 \mathrm{EN} ; 30 \times 0]$} & $158,1-153,1 \quad(d, C 1)$ & \\
\hline & $127,3-125,1 \quad(d, C 2)$ & 100,0 \\
\hline & $187,6-183,6(s, C 3)$ & \\
\hline & $133,1-129,1 \quad(s, C 4)$ & \\
\hline & $160,5-153,8(\mathrm{~s}, \mathrm{C} 5)$ & \\
\hline
\end{tabular}


Tabela 16. Continuação

\begin{tabular}{|c|c|c|}
\hline Subesqueleto & $\begin{array}{l}\text { Faixas de deslocamentos } \\
\text { químicos (ppm) }\end{array}$ & $\begin{array}{c}\text { Percentual de } \\
\text { Reconhecimento (\%) }\end{array}$ \\
\hline & $151,1-147,1 \quad(s, C 4)$ & \\
\hline & $49,7-43,5(d, C 5)$ & \\
\hline \multirow[t]{5}{*}{$1[4(14) \mathrm{EN} ; 11 \mathrm{OH}]$} & $40,9-24,2(t, C 6)$ & 81,8 \\
\hline & $59,0-47,5(d, C 7)$ & \\
\hline & $80,6-71,5(s, \mathrm{c} 11)$ & \\
\hline & $109,0-105,3(t, C 14)$ & \\
\hline & $200,3-186,5(s, C 3)$ & \\
\hline \multirow[t]{5}{*}{ 1[3OXO; 11OR; 4EN] } & $131,3-125,9(\mathrm{~s}, \mathrm{C} 4)$ & 100,0 \\
\hline & $164,1-158,8(\mathrm{~s}, \mathrm{C} 5)$ & \\
\hline & $50,5-49,5(d, C 7)$ & \\
\hline & $73,4-72,0(s, \mathrm{C} 11)$ & \\
\hline & $200,3-180,8(5, C 3)$ & \\
\hline \multirow[t]{4}{*}{$1[30 \times 0 ; 4 E N]$} & $133,8-125,9(s, C 4)$ & 100,0 \\
\hline & $164,1-150,1 \quad(s, C 5)$ & \\
\hline & $42,4-35,0(s, C 10)$ & \\
\hline & $31,5-23,7(\mathrm{t}, \mathrm{C} 6)$ & \\
\hline \multirow[t]{6}{*}{$1[11 \mathrm{EN} ; 130 \times 0 ; 130 \mathrm{OR}]$} & $40,4-35,9(d, C 7)$ & 94,0 \\
\hline & $38,5-25,1 \quad(t, C 8)$ & \\
\hline & $149,5-132,8(s, \mathrm{C} 11)$ & \\
\hline & $125,1-121,9(t, C 12)$ & \\
\hline & $172,3-167,1(s, C 13)$ & \\
\hline & $91,3-76,3(d, C 1)$ & \\
\hline \multirow[t]{4}{*}{$1[1,11 \mathrm{OH}]$} & $48,9-45,4(d, C 5)$ & 100,0 \\
\hline & $40,2-36,2(s, C 10)$ & \\
\hline & $75,1-72,5$ (s, C11) & \\
\hline & $77,0-72,0(\mathrm{~s}, \mathrm{C} 4)$ & \\
\hline \multirow[t]{4}{*}{$1[4,11 \mathrm{OR}]$} & $54,9-47,2(d, C 5)$ & 100,0 \\
\hline & $21,6-20,5(t, C 6)$ & \\
\hline & $50,7-41,0(\mathrm{~d}, \mathrm{C} 7)$ & \\
\hline & $85,0-73,0(s, C 11)$ & \\
\hline
\end{tabular}


Tabela 15. Continuação

\begin{tabular}{|c|c|c|}
\hline Subesqueleto & $\begin{array}{l}\text { Faixas de deslocamentos } \\
\text { químicos (ppm) }\end{array}$ & $\begin{array}{c}\text { Percentual de } \\
\text { Reconhecimento (\%) } \\
\end{array}$ \\
\hline & $91,3-80,0(d, C 1)$ & \\
\hline \multirow[t]{5}{*}{$1[1 \mathrm{OR} ; 4,11 \mathrm{OH}]$} & $72,8-72,0(\mathrm{~s}, \mathrm{C} 4)$ & 100,0 \\
\hline & $48,5-47,2(d, C 5)$ & \\
\hline & $40,2-38,7(s, C 10)$ & \\
\hline & $75,1-74,5(\mathrm{~s}, \mathrm{C} 11)$ & \\
\hline & $84,3-71,0(s, C 4)$ & \\
\hline \multirow[t]{5}{*}{$1[4,6 \mathrm{OR}]$} & $57,7-45,7(d, C 5)$ & 78,9 \\
\hline & $77,9-66,3(d, c 6)$ & \\
\hline & $51,2-43,2(d, C 7)$ & \\
\hline & $29,7-23,5$ (d, C11) & \\
\hline & $52,4-46,4 \quad(d, C 2)$ & \\
\hline \multirow[t]{5}{*}{$94[$ sem substituintes] } & $85,9-72,0 \quad(s, C 3)$ & 100,0 \\
\hline & $87,3-69,5(s, C 7)$ & \\
\hline & $40,4-34,0(d, C 9)$ & \\
\hline & $38,5-37,7(s, \mathrm{C} 11)$ & \\
\hline & $140,5-124,1 \quad(d, C 5)$ & \\
\hline \multirow[t]{4}{*}{$64[4 E N ; 100 \times 0]$} & $135,3-131,6(s, C 4)$ & 100,0 \\
\hline & $65,5-60,2(s, \mathrm{c} 6)$ & \\
\hline & $217,3-214,8(s, C 10)$ & \\
\hline & $45,5-40,0(d, C 1)$ & \\
\hline \multirow[t]{4}{*}{64 [sem substituintes] } & $65,5-60,2(s, \mathrm{c} 6)$ & 100,0 \\
\hline & $35,4-32,5(d, c 9)$ & \\
\hline & $38,9-36,2(d, C 11)$ & \\
\hline & $58,2-33,5(d, C 1)$ & \\
\hline \multirow[t]{4}{*}{$122[8(14) \mathrm{EN}]$} & $158,1-148,3(s, C 8)$ & 92,6 \\
\hline & $56,4-36,2(d, C 9)$ & \\
\hline & $42,0-27,2(t, C 10)$ & \\
\hline & $40,0-32,7(s, C 11)$ & \\
\hline
\end{tabular}


Tabela 15. Continuação

\begin{tabular}{|c|c|c|}
\hline Subesqueleto & $\begin{array}{c}\text { Faixas de deslocamentos } \\
\text { químicos (ppm) } \\
\end{array}$ & $\begin{array}{c}\text { Percentual de } \\
\text { Reconhecimento (\%) } \\
\end{array}$ \\
\hline & $53,5-39,9(d, C 1)$ & \\
\hline \multirow[t]{6}{*}{$122[4,5 \mathrm{EP}]$} & $63,7-57,9(\mathrm{~s}, \mathrm{C} 4)$ & 100,0 \\
\hline & $68,8-59,2(d, C 5)$ & \\
\hline & $56,4-42,2$ (d, C9) & \\
\hline & $40,2-28,7(t, C 10)$ & \\
\hline & $39,9-32,7$ (s, C11) & \\
\hline & $146,8-127,5(\mathrm{~s}, \mathrm{C} 4)$ & \\
\hline \multirow[t]{5}{*}{$122[4,8(14) \mathrm{EN}]$} & $146,6-116,5(d, C 5)$ & 100,0 \\
\hline & $154,3-149,3(\mathrm{~s}, \mathrm{C} 8)$ & \\
\hline & $40,0-32,9(s, 11)$ & \\
\hline & $126,4-110,4(t, C 14)$ & \\
\hline & $126,1-125,1 \quad(d, C 3)$ & \\
\hline \multirow[t]{3}{*}{$122[3 E N]$} & $138,0-137,5(\mathrm{~s}, \mathrm{C} 4)$ & 100,0 \\
\hline & $38,5-33,0(s, \mathrm{c} 11)$ & \\
\hline & $58,2-52,0(d, C 1)$ & \\
\hline \multirow[t]{5}{*}{$122[150 \times O ; 15 O R]$} & $45,2-40,2(d, C 9)$ & 100,0 \\
\hline & $42,0-29,3(t, C 10)$ & \\
\hline & $34,7-33,2$ (s, C11) & \\
\hline & $176,8-168,5(\mathrm{~s}, \mathrm{C} 15)$ & \\
\hline & $48,2-46,2(s, C 5)$ & \\
\hline \multirow[t]{3}{*}{$73[140 \times 0]$} & $58,5-54,2(d, C 10)$ & 100,0 \\
\hline & $206,3-204,3(d, C 14)$ & \\
\hline & $126,0-122,0(\mathrm{~d}, \mathrm{C} 1)$ & \\
\hline \multirow[t]{4}{*}{$73[1(10) E N ; 2 O X O]$} & $199,3-186,3(s, C 2)$ & 100,0 \\
\hline & $52,7-50,0(s, C 5)$ & \\
\hline & $172,3-164,3(s, 10)$ & \\
\hline & $52,7-46,2(s, C 5)$ & \\
\hline \multirow[t]{3}{*}{$73[11 \mathrm{EN}]$} & $49,2-37,5(d, C 7)$ & 92,0 \\
\hline & $150,8-146,1 \quad(s, C 11)$ & \\
\hline & $109,5-107,8(t, C 12)$ & \\
\hline
\end{tabular}


Tabela 15. Continuação

\begin{tabular}{|c|c|c|}
\hline Subesqueleto & $\begin{array}{c}\text { Faixas de deslocamentos } \\
\text { químicos (ppm) }\end{array}$ & $\begin{array}{c}\text { Percentual de } \\
\text { Reconhecimento (\%) } \\
\end{array}$ \\
\hline & $73,6-66,0(\mathrm{~d}, \mathrm{C} 2)$ & \\
\hline \multirow[t]{3}{*}{$73[2,14 \mathrm{OR}]$} & $48,0-46,2(\mathrm{~s}, \mathrm{C} 5)$ & 100,0 \\
\hline & $66,3-61,2$ (t, C14) & \\
\hline & $28,6-24,6(t, C 1)$ & \\
\hline \multirow[t]{4}{*}{59 [sem substituintes] } & $35,0-31,8(d, C 3)$ & 100,0 \\
\hline & $60,2-58,7(d, C 4)$ & \\
\hline & $48,0-45,7$ (s, c9) & \\
\hline & $60,2-58,7(d, C 4)$ & \\
\hline \multirow[t]{4}{*}{$59[5,80 R]$} & $72,4-71,5(\mathrm{~s}, \mathrm{C5})$ & 100,0 \\
\hline & $81,3-79,8(\mathrm{~d}, \mathrm{C} 8)$ & \\
\hline & $48,0-45,7(s, c 9)$ & \\
\hline & $45,2-44,5(s, C 5)$ & \\
\hline \multirow[t]{3}{*}{$65[7 \mathrm{EN}]$} & $122,5-120,3(d, C 7)$ & 100,0 \\
\hline & $137,3-134,0(\mathrm{~s}, \mathrm{C} 8)$ & \\
\hline & $51,5-43,7(d, C 5)$ & \\
\hline \multirow[t]{5}{*}{$91[10 \mathrm{OH}]$} & $38,2-36,0(\mathrm{~s}, \mathrm{C} 6)$ & 100,0 \\
\hline & $76,0-73,8 \quad(s, \mathrm{C} 10)$ & \\
\hline & $52,5-44,0(d, C 11)$ & \\
\hline & 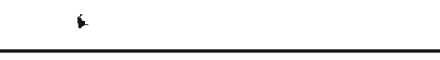 & \\
\hline & $143,3-128,6(d, C 2)$ & \\
\hline \multirow[t]{4}{*}{$91[2 E N]$} & $136,5-135,1 \quad(s, C 3)$ & 100,0 \\
\hline & $51,5-46,2(d, C 5)$ & \\
\hline & $37,0-36,0(\mathrm{~s}, \mathrm{C} 6)$ & \\
\hline & $135,0-132,0(\mathrm{~s}, \mathrm{C} 3)$ & \\
\hline \multirow[t]{4}{*}{$91[3 E N]$} & $131,0-125,0(d, C 4)$ & 100,0 \\
\hline & $47,0-43,0(d, C 5)$ & \\
\hline & $39,0-37,0(\mathrm{~s}, \mathrm{C} 6)$ & \\
\hline & $209,6-198,8(s, C 1)$ & \\
\hline \multirow[t]{3}{*}{$79[10 \times O ; 2 E N]$} & $128,8-126,5(d, C 2)$ & 100,0 \\
\hline & $158,1-145,1 \quad(s, C 3)$ & \\
\hline & $65,0-54,7(s, C 7)$ & \\
\hline
\end{tabular}


Tabela 15. Continuação

\begin{tabular}{|c|c|c|}
\hline Subesqueleto & $\begin{array}{c}\text { Faixas de deslocamentos } \\
\text { químicos }(\mathrm{ppm})\end{array}$ & $\begin{array}{c}\text { Percentual de } \\
\text { Reconhecimento } \\
\end{array}$ \\
\hline & $155,1-123,6(d, C 2)$ & \\
\hline \multirow{5}{*}{$79[5 \mathrm{OR} ; 100 \mathrm{OH} ; 2 \mathrm{EN}]$} & $151,1-125,5(\mathrm{~s}, \mathrm{C} 3)$ & 100,0 \\
\hline & $79,8-67,9(d, C 5)$ & \\
\hline & $55,7-43,2(s, C 7)$ & \\
\hline & $87,9-80,9(\mathrm{~s}, \mathrm{C} 10)$ & \\
\hline & $62,4-58,2(d, c 2)$ & \\
\hline \multirow[t]{3}{*}{$79[2,3 E P]$} & $58,2-56,0(\mathrm{~s}, \mathrm{C} 3)$ & 100,0 \\
\hline & $48,7-43,4(s, C 7)$ & \\
\hline & $78,3-75,0 \quad(s, \mathrm{c} 6)$ & \\
\hline \multirow[t]{4}{*}{$79[6,10 \mathrm{EP}]$} & $55,7-41,9(\mathrm{~s}, \mathrm{C} 7)$ & 100,0 \\
\hline & $76,8-67,0$ (s, C10) & \\
\hline & $33,9-28,5$ (d, C11) & \\
\hline & $87,4-83,5 \quad(s, \mathrm{c} 3)$ & \\
\hline \multirow[t]{3}{*}{$79[3,60 \times 1]$} & $92,1-90,4(s, \mathrm{C} 6)$ & 100,0 \\
\hline & $45,9-44,7(\mathrm{~s}, \mathrm{C} 7)$ & \\
\hline & $129,3-119,4(d, C 2)$ & \\
\hline \multirow[t]{5}{*}{$79[2 \mathrm{EN} ; 8 \mathrm{OXO}]$} & $146,3-133,8(s, \mathrm{C} 3)$ & 83,3 \\
\hline & $60,2-43,2(s, \mathrm{C} 7)$ & \\
\hline & $220,1-217,3(s, C 8)$ & \\
\hline & $61,9-26,2(d, C 11)$ & \\
\hline & $212,8-206,0(s, C 2)$ & \\
\hline \multirow[t]{5}{*}{$79[20 \times 0 ; 6(10) E N]$} & $176,0-148,3(\mathrm{~s}, \mathrm{C} 6)$ & 100,0 \\
\hline & $46,0-41,4(s, \mathrm{c} 7)$ & \\
\hline & $176,6-140,5(s, C 10)$ & \\
\hline & $26,5-24,8$ (d, C11) & \\
\hline & $50,2-42,2(s, \mathrm{c} 7)$ & \\
\hline \multirow[t]{3}{*}{$79[2 \mathrm{EN} ; 14 \mathrm{OXO}]$} & $155,1-150,8(d, C 2)$ & 100,0 \\
\hline & $148,6-140,1 \quad(s, c 3)$ & \\
\hline & $195,0-191,8(d, C 14)$ & \\
\hline
\end{tabular}


Tabela 6. Continuação

\begin{tabular}{|c|c|c|}
\hline Subesqueleto & $\begin{array}{c}\text { Faixas de deslocamentos } \\
\text { químicos (ppm) }\end{array}$ & $\begin{array}{c}\text { Percentual de } \\
\text { Reconhecimento (\%) } \\
\end{array}$ \\
\hline & $154,6-122,8(d, C 2)$ & \\
\hline \multirow[t]{4}{*}{$79[2,11 \mathrm{EN}]$} & $148,6-134,0 \quad(s, C 3)$ & 100,0 \\
\hline & $50,2-42,2(s, c 7)$ & \\
\hline & $148,1-141,8 \quad(s, \mathrm{C} 11)$ & \\
\hline & $113,9-110,8(t, C 12)$ & \\
\hline & $176,0-145,1(\mathrm{~s}, \mathrm{C} 6)$ & \\
\hline \multirow[t]{4}{*}{$79[6(10) \mathrm{EN}]$} & $60,7-41,4(\mathrm{~s}, \mathrm{CT})$ & 83,3 \\
\hline & $176,6-132,8(\mathrm{~s}, \mathrm{C} 10)$ & \\
\hline & $27,7-24,8$ (d, C11) & \\
\hline & $146,6-145,1 \quad(s, C 6)$ & \\
\hline \multirow[t]{4}{*}{$79[6(10) \mathrm{EN}]$} & $60,7-49,7(s, c 7)$ & 100,0 \\
\hline & $32,5-27,3(\mathrm{t}, \mathrm{Cg})$ & \\
\hline & $141,0-132,8(\mathrm{~s}, \mathrm{C} 10)$ & \\
\hline & $56,0-29,1(t, C 5)$ & \\
\hline \multirow[t]{5}{*}{$79[5,6 \mathrm{EP}]$} & $85,5-81,5(\mathrm{~s}, \mathrm{C} 6)$ & 84,2 \\
\hline & $65,0-46,5(\mathrm{~s}, \mathrm{c})$ & \\
\hline & $58,9-50,9(\mathrm{~d}, \mathrm{C} 10)$ & \\
\hline & $29,3-26,2(d, C 11)$ & \\
\hline & $41,0-36,0 \quad(s, C 5)$ & \\
\hline \multirow[t]{6}{*}{$6[9 \mathrm{EN}]$} & $47,0-31,2(t, C 6)$ & 78,9 \\
\hline & $41,5-33,2(d, c 7)$ & \\
\hline & $39,9-22,6(t, C 8)$ & \\
\hline & $135,3-118,9$ (d, C9) & \\
\hline & $144,3-138,6(s, C 10)$ & \\
\hline & $41,0-38,7(s, C 5)$ & \\
\hline \multirow[t]{5}{*}{$6[9,11 \mathrm{EN}]$} & $39,9-29,8(t, C 8)$ & 100,0 \\
\hline & $135,3-118,9$ (d, C9) & \\
\hline & $144,3-138,6(s, C 10)$ & \\
\hline & $154,6-148,0 \quad(s, \mathrm{C} 11)$ & \\
\hline & $123,5-108,1 \quad(t, C 12)$ & \\
\hline
\end{tabular}


Tabela 15. Continuação

\begin{tabular}{|c|c|c|}
\hline Subesqueleto & $\begin{array}{c}\text { Faixas de deslocamentos } \\
\text { químicos (ppm) } \\
\end{array}$ & $\begin{array}{c}\text { Percentual de } \\
\text { Reconhecimento (\%) } \\
\end{array}$ \\
\hline & $50,5-43,2(d, c 7)$ & \\
\hline \multirow[t]{6}{*}{$6[80 \times 0 ; 9,11 \mathrm{EN}]$} & $198,5-197,6(\mathrm{~s}, \mathrm{C} 8)$ & 100,0 \\
\hline & $129,6-123,4(d, C 9)$ & \\
\hline & $167,3-160,6$ (s, C10) & \\
\hline & $143,3-142,8$ (s, C11) & \\
\hline & $144,3-113,5$ (t, C12) & \\
\hline & $139,8-127,0 \quad(s, C 7)$ & \\
\hline \multirow[t]{3}{*}{$6[7(11) \mathrm{EN} ; 80 \times 0]$} & $205,3-191,6(\mathrm{~s}, \mathrm{C} 8)$ & 100,0 \\
\hline & $143,3-131,1 \quad(s, C 11)$ & \\
\hline & $128,1-126,0 \quad(\mathrm{~s}, \mathrm{c} 7)$ & \\
\hline \multirow[t]{5}{*}{$6[7(11), 9 \mathrm{EN} ; 80 \times 0]$} & $192,6-190,6(s, C 8)$ & 100,0 \\
\hline & $127,6-125,5(d, C 9)$ & \\
\hline & $167,1-164,0(\mathrm{~s}, \mathrm{C} 10)$ & \\
\hline & $144,3-142,1 \quad(s, \mathrm{C} 11)$ & \\
\hline & $148,6-145,5(d, C 1)$ & \\
\hline \multirow[t]{5}{*}{$6[1 \mathrm{EN} ; 30 \times O]$} & $131,6-122,9(\mathrm{~d}, \mathrm{C} 2)$ & 100,0 \\
\hline & $202,6-200,6(\mathrm{~s}, \mathrm{C} 3)$ & \\
\hline & $55,2-47,2(t, C 4)$ & \\
\hline & $41,0-39,7(\mathrm{~s}, \mathrm{C} 5)$ & \\
\hline & $54,5-43,2(\mathrm{~d}, \mathrm{C} 7)$ & \\
\hline \multirow[t]{4}{*}{$6[11 \mathrm{EN} ; 80 \times 0]$} & $209,8-197,6(s, C 8)$ & 71,4 \\
\hline & $143,3-141,8$ (s, C11) & \\
\hline & $144,3-113,5(t, C 12)$ & \\
\hline & $40,2-36,9(\mathrm{~s}, \mathrm{C5})$ & \\
\hline \multirow[t]{6}{*}{$6[80 \times 0 ; 9 E N]$} & $42,4-37,5(t, C 6)$ & 71,4 \\
\hline & $50,5-43,2(d, C 7)$ & \\
\hline & $202,8-197,6(s, C 8)$ & \\
\hline & $129,6-123,4(d, C 9)$ & \\
\hline & $168,1-160,6(\mathrm{~s}, \mathrm{C} 10)$ & \\
\hline & $42,7-41,7(\mathrm{~d}, \mathrm{C} 4)$ & \\
\hline \multirow[t]{3}{*}{$6[7,11 E P]$} & $38,5-37,2(s, \mathrm{C} 5)$ & 100,0 \\
\hline & $67,8-61,0 \quad(s, C 7)$ & \\
\hline & $72,0-64,0 \quad(\mathrm{~s}, \mathrm{C} 11)$ & \\
\hline
\end{tabular}


Tabela 16. Continuação

\begin{tabular}{|c|c|c|}
\hline Subesqueleto & $\begin{array}{l}\text { Faixas de deslocamentos } \\
\text { químicos (ppm) }\end{array}$ & $\begin{array}{c}\text { Percentual de } \\
\text { Reconhecimento (\%) }\end{array}$ \\
\hline & $58,2-55,9(\mathrm{~d}, \mathrm{C} 1)$ & \\
\hline \multirow[t]{4}{*}{$6[1,2 \mathrm{EP}]$} & $57,9-50,2(d, C 2)$ & 100,0 \\
\hline & $45,7-41,7(d, C 4)$ & \\
\hline & $38,5-36,9(s, C 5)$ & \\
\hline & $64,0-60,5(d, C 1)$ & \\
\hline \multirow[t]{4}{*}{$6[1,10] E P$} & $41,5-32,0(d, C 4)$ & 100,0 \\
\hline & $45,0-35,9(\mathrm{~s}, \mathrm{C5})$ & \\
\hline & $65,5-63,7(s, C 10)$ & \\
\hline & $32,5-30,1 \quad(t, C 1)$ & \\
\hline \multirow[t]{7}{*}{$6[9 \mathrm{EN}]$} & $39,2-38,5(s, C 5)$ & 100,0 \\
\hline & $44,2-38,5(d, C 6)$ & \\
\hline & $41,5-37,7(d, C 7)$ & \\
\hline & $31,2-22,6(t, C 8)$ & \\
\hline & $122,9-120,0(d, C 9)$ & \\
\hline & $144,3-138,6(s, C 10)$ & \\
\hline & $75,5-67,1 \quad(d, c 1)$ & \\
\hline \multirow[t]{4}{*}{$6[1 \mathrm{OH} ; 9 \mathrm{EN}]$} & $40,2-38,7(s, C 5)$ & 100,0 \\
\hline & $129,3-118,9(d, C 9)$ & \\
\hline & $141,3-140,0(s, C 10)$ & \\
\hline & $73,5-68,1 \quad(d, C 1)$ & \\
\hline \multirow[t]{5}{*}{$6[30 R ; 80 \times 0 ; 9 E N]$} & $42,2-36,9(\mathrm{~s}, \mathrm{C} 5)$ & 92,9 \\
\hline & $202,8-190,6(s, C 8)$ & \\
\hline & $131,5-121,0(\mathrm{~d}, \mathrm{C} 9)$ & \\
\hline & $169,1-160,6(s, C 10)$ & \\
\hline & $51,4-35,2(s, C 5)$ & \\
\hline \multirow[t]{4}{*}{$6[8,12$ FURANO $]$} & $137,3-115,4(s, c 7)$ & 91,0 \\
\hline & $164,1-144,8(s, C 8)$ & \\
\hline & $121,6-116,4$ (s, C11) & \\
\hline & $145,5-137,0(\mathrm{~d}, \mathrm{C} 12)$ & \\
\hline
\end{tabular}


Tabela 15. Continuação

\begin{tabular}{|c|c|c|}
\hline Subesqueleto & $\begin{array}{c}\text { Faixas de deslocamentos } \\
\text { químicos (ppm) } \\
\end{array}$ & $\begin{array}{c}\text { Percentual de } \\
\text { Reconhecimento (\%) }\end{array}$ \\
\hline & $137,3-133,6(s, c 7)$ & \\
\hline \multirow[t]{5}{*}{$6[8,12$ FURANO; $90 \times 0]$} & $146,6-144,8 \quad(\mathrm{~s}, \mathrm{C} 8)$ & 100,0 \\
\hline & $186,5-183,8(s, C 9)$ & \\
\hline & $121,6-120,6(s, C 11)$ & \\
\hline & $145,5-144,3(d, C 12)$ & \\
\hline & $63,4-59,0(d, c 1)$ & \\
\hline \multirow[t]{6}{*}{$6[1,10 \mathrm{EP} ; 8,12 \mathrm{FURANO}]$} & $120,0-117,0(s, \mathrm{c} 7)$ & 100,0 \\
\hline & $162,3-146,3(s, C 8)$ & \\
\hline & $65,9-62,7\left(s, C_{10}\right)$ & \\
\hline & $119,5-116,4$ (s, C11) & \\
\hline & $140,1-137,6(d, C 12)$ & \\
\hline & $132,8-114,3(s, C 1)$ & \\
\hline \multirow[t]{6}{*}{$10[1(6), 2,4,7,9 \mathrm{EN}]$} & $133,6-126,4(s, C 4)$ & 100,0 \\
\hline & $125,0-115,8(d, C 5)$ & \\
\hline & $142,1-125,0(\mathrm{~s}, \mathrm{C} 6)$ & \\
\hline & $144,8-130,3(s, C 7)$ & \\
\hline & $127,3-111,0(s, \mathrm{C} 10)$ & \\
\hline & $162,3-161,6(s, C 5)$ & \\
\hline \multirow[t]{3}{*}{$10[1(6), 5,7,9 \mathrm{EN} ; 7,110 \mathrm{OI}]$} & $127,4-125,5(s, C 6)$ & 100,0 \\
\hline & $126,9-119,0(\mathrm{~s}, \mathrm{C} 7)$ & \\
\hline & $182,3-180,1 \quad(s, C 2)$ & \\
\hline \multirow[t]{6}{*}{$10[1(6), 5,7,9 \mathrm{EN} ; 2,3 \mathrm{OXO}]$} & $180,3-177,8(s, \mathrm{c} 3)$ & 100,0 \\
\hline & $116,3-112,1(s, C 4)$ & \\
\hline & $162,3-161,6(s, \mathrm{C} 5)$ & \\
\hline & $127,4-125,5(\mathrm{~s}, \mathrm{C} 6)$ & \\
\hline & $126,9-119,0(\mathrm{~s}, \mathrm{C} 7)$ & \\
\hline & $117,0-114,3(\mathrm{~s}, \mathrm{C} 1)$ & \\
\hline \multirow[t]{4}{*}{$10[1(6), 2,4,7,9 \mathrm{EN} ; 2 \mathrm{OR}]$} & $151,8-144,3(s, C 2)$ & 100,0 \\
\hline & $129,3-125,0(s, \mathrm{C} 6)$ & \\
\hline & $144,8-132,8(\mathrm{~s}, \mathrm{C} 7)$ & \\
\hline & $113,0-111,0(s, C 10)$ & \\
\hline
\end{tabular}


Tabela 15. Continuação

\begin{tabular}{|c|c|c|}
\hline Subesqueleto & $\begin{array}{c}\text { Faixas de deslocamentos } \\
\text { químicos (ppm) } \\
\end{array}$ & $\begin{array}{c}\text { Percentual de } \\
\text { Reconhecimento (\%) }\end{array}$ \\
\hline & $151,8-144,3(s, \mathrm{C} 2)$ & \\
\hline \multirow[t]{5}{*}{$10[1(6), 2,4,7,9 \mathrm{EN} ; 2,8,9 \mathrm{OR} ; 14 \mathrm{OXO}]$} & $146,5-141,8(s, C 8)$ & 100,0 \\
\hline & $160,3-155,0 \quad(s, c 9)$ & \\
\hline & $113,0-111,0(s, \mathrm{C} 10)$ & \\
\hline & $199,3-198,3$ (d, C14) & \\
\hline & $142,1-126,5(s, C 1)$ & \\
\hline \multirow[t]{6}{*}{$10[1(6), 2,4 \mathrm{EN}]$} & $135,1-120,5(\mathrm{~s}, \mathrm{C} 4)$ & 88,9 \\
\hline & $130,5-122,6(d, C 5)$ & \\
\hline & $142,1-131,3(s, \mathrm{C} 6)$ & \\
\hline & $30,8-27,2(t, c 9)$ & \\
\hline & $33,0-26,7(d, C 10)$ & \\
\hline & $128,6-126,5(s, C 1)$ & \\
\hline \multirow[t]{5}{*}{$10[1(6), 2,4 \mathrm{EN} ; 1 \mathrm{OR}]$} & $157,1-150,8(s, C 2)$ & 100,0 \\
\hline & $113,8-108,5(d, C 3)$ & \\
\hline & $135,1-119,5(\mathrm{~s}, \mathrm{C} 4)$ & \\
\hline & $26,7-26,6(\mathrm{~d}, \mathrm{C} 10)$ & \\
\hline & $142,1-132,1 \quad(s, C 1)$ & \\
\hline \multirow[t]{5}{*}{$10[1(6), 2,4 \mathrm{EN} ; 3 \mathrm{OR}]$} & $113,0-108,4(d, C 2)$ & 100,0 \\
\hline & $156,3-151,3(s, C 3)$ & \\
\hline & $123,5-120,5(\mathrm{~s}, \mathrm{C} 4)$ & \\
\hline & $33,0-32,5\left(d, C_{10}\right)$ & \\
\hline & $136,8-125,5(s, C 1)$ & \\
\hline \multirow[t]{5}{*}{$10[20 \times 0 ; 1(6), 7,9 E N]$} & $186,3-180,1 \quad(s, C 2)$ & 100,0 \\
\hline & $132,3-125,5(\mathrm{~s}, \mathrm{C} 6)$ & \\
\hline & $150,6-119,0 \quad(s, C 7)$ & \\
\hline & $146,5-115,8(s, c 10)$ & \\
\hline & $72,6-70,6(s, \mathrm{C} 1)$ & \\
\hline \multirow[t]{5}{*}{$10[10 \mathrm{OH} ; 4 \mathrm{EN}]$} & $31,2-26,7(t, C 2)$ & 100,0 \\
\hline & $31,2-22,1(t, C 3)$ & \\
\hline & $135,1-133,6(s, C 4)$ & \\
\hline & $123,3-119,8(d, C 5)$ & \\
\hline & $49,2-39,9(d, C 6)$ & \\
\hline
\end{tabular}


Tabela 15. Continuação

\begin{tabular}{|c|c|c|}
\hline Subesqueleto & $\begin{array}{c}\text { Faixas de deslocamentos } \\
\text { químicos (ppm) } \\
\end{array}$ & $\begin{array}{c}\text { Percentual de } \\
\text { Reconhecimento (\%) } \\
\end{array}$ \\
\hline & $85,6-84,9(\mathrm{~s}, \mathrm{C} 1)$ & \\
\hline \multirow[t]{5}{*}{$10[1,80 X \mathrm{O} ;$ 4EN $]$} & $131,1-130,6(\mathrm{~s}, \mathrm{C} 4)$ & 100,0 \\
\hline & $125,1-124,8(d, C 5)$ & \\
\hline & $49,5-41,0(d, C 6)$ & \\
\hline & $78,0-77,5(d, c 8)$ & \\
\hline & $49,5-28,7$ (d, C1) & \\
\hline \multirow[t]{7}{*}{$10[4,11 \mathrm{EN}]$} & $141,3-129,1 \quad(s, C 4)$ & 100,0 \\
\hline & $153,0-124,9(d, C 5)$ & \\
\hline & $152,8-143,3$ (s, C11) & \\
\hline & $51,0-39,4(d, c 6)$ & \\
\hline & $45,4-36,5(d, c 7)$ & \\
\hline & $125,3-108,1 \quad(t, C 12)$ & \\
\hline & $44,4-42,7(d, C 7)$ & \\
\hline \multirow[t]{4}{*}{$10[11 \mathrm{EN} ; 13 \mathrm{OH}]$} & $66,4-65,4$ (t, C13) & 100,0 \\
\hline & $111,8-108,1 \quad(t, C 12)$ & \\
\hline & $152,8-146,8 \quad(s, C 11)$ & \\
\hline & $148,8-124,3(s, C 1)$ & \\
\hline \multirow[t]{5}{*}{$10[1(10), 4 \mathrm{EN}]$} & $137,6-124,0 \quad(\mathrm{~s}, \mathrm{C} 4)$ & 100,0 \\
\hline & $144,3-123,1 \quad(d, C 5)$ & \\
\hline & $41,0-39,2(d, C 6)$ & \\
\hline & $131,1-120,0(s, c 10)$ & \\
\hline & $95,8-95,5(\mathrm{~s}, \mathrm{C} 5)$ & \\
\hline \multirow[t]{2}{*}{$10[5,110 \times 1 ; 5 E N]$} & $166,8-166,3(s, \mathrm{C} 11)$ & 100,0 \\
\hline & $206,1-202,1 \quad(s, C 3)$ & \\
\hline \multirow[t]{5}{*}{$10[1,9 \mathrm{EN} ; 3 \mathrm{OXO}]$} & $139,1-135,1 \quad(d, C 2)$ & 100,0 \\
\hline & $161,1-157,1 \quad(s, C 1)$ & \\
\hline & $133,6-129,6(\mathrm{~s}, \mathrm{C} 10)$ & \\
\hline & $122,1-118,1 \quad(d, C 9)$ & \\
\hline & $199,8-182,0 \quad(s, C 3)$ & \\
\hline \multirow[t]{2}{*}{$10[30 \times 0 ; 4 E N]$} & $135,8-134,5(\mathrm{~s}, \mathrm{C} 4)$ & 100,0 \\
\hline & $146,8-137,8(d, C 5)$ & \\
\hline
\end{tabular}


Tabela 15. Continuação

\begin{tabular}{|c|c|c|}
\hline Subesqueleto & $\begin{array}{c}\text { Faixas de deslocamentos químicos } \\
(\mathrm{ppm})\end{array}$ & $\begin{array}{c}\text { Percentual de } \\
\text { Reconhecimento (\%) } \\
\end{array}$ \\
\hline & $69,3-67,6(\mathrm{~s}, \mathrm{C} 4)$ & \\
\hline \multirow[t]{3}{*}{$10[4 \mathrm{OH} ; 5 \mathrm{EN}]$} & $131,6-128,1 \quad(d, C 5)$ & 100,0 \\
\hline & $144,1-137,0(\mathrm{~s}, \mathrm{C} 6)$ & \\
\hline & $48,2-46,7(d, C 1)$ & \\
\hline \multirow[t]{4}{*}{$10[10 \mathrm{NCS}]$} & $66,0-61,2(\mathrm{~s}, \mathrm{C} 10)$ & 100,0 \\
\hline & $42,7-37,7 \quad(t, C 9)$ & \\
\hline & $23,0-22,0 \quad(t, C 8)$ & \\
\hline & $51,4-50,0(d, c 7)$ & \\
\hline \multirow[t]{5}{*}{$10[4 \mathrm{EN} ; 110 \mathrm{H}]$} & $73,6-72,6(\mathrm{~s}, \mathrm{c} 11)$ & 100,0 \\
\hline & $46,5-38,0(\mathrm{~d}, \mathrm{C} 6)$ & \\
\hline & $141,8-129,6(s, C 4)$ & \\
\hline & $154,5-122,1 \quad(d, C 5)$ & \\
\hline & $46,5-38,0(d, C 6)$ & \\
\hline \multirow[t]{4}{*}{$10[11 \mathrm{OH}]$} & $51,4-50,0(d, C 7)$ & 100,0 \\
\hline & $23,7-22,8(t, C 8)$ & \\
\hline & $73,6-72,6(\mathrm{~s}, \mathrm{C} 11)$ & \\
\hline & $79,1-78,9(\mathrm{~d}, \mathrm{C9})$ & \\
\hline \multirow[t]{4}{*}{$10[8,90 \mathrm{R} ; 10(14) \mathrm{EN}]$} & $79,1-79,0 \quad(d, C 9)$ & 100,0 \\
\hline & $103,1-103,0 \quad(t, C 14)$ & \\
\hline & $153,3-152,1 \quad(s, \mathrm{C} 10)$ & \\
\hline & $79,1-67,8(d, C 8)$ & \\
\hline \multirow[t]{7}{*}{$10[4,10(14) \mathrm{EN} ; 8 \mathrm{OH}]$} & $111,5-103,0$ (t, C14) & 100,0 \\
\hline & $153,0-148,3(s, C 10)$ & \\
\hline & $128,6-122,0 \quad(d, C 5)$ & \\
\hline & $139,8-134,1 \quad(s, C 4)$ & \\
\hline & $55,2-44,7(d, C 7)$ & \\
\hline & $111,5-103,0(t, C 14)$ & 100,0 \\
\hline & $154,3-148,3(\mathrm{~s}, \mathrm{C} 10)$ & \\
\hline \multirow[t]{4}{*}{$10[4 ; 10(14) \mathrm{EN}]$} & $44,7-37,4(\mathrm{~d}, \mathrm{C} 1)$ & \\
\hline & $44,9-39,7(d, C 6)$ & \\
\hline & $128,6-122,0(d, C 5)$ & \\
\hline & $139,8-133,8(\mathrm{~s}, \mathrm{C} 4)$ & \\
\hline
\end{tabular}


Tabela 16. Continuação

\begin{tabular}{|c|c|c|}
\hline Subesqueleto & $\begin{array}{c}\text { Faixas de deslocamentos } \\
\text { químicos (ppm) } \\
\end{array}$ & $\begin{array}{c}\text { Percentual de } \\
\text { Reconhecimento (\%) } \\
\end{array}$ \\
\hline & $32,8-29,5(d, C 11)$ & \\
\hline \multirow[t]{5}{*}{$10[4 \mathrm{EN} ; 7 \mathrm{OR}]$} & $76,0-74,9(\mathrm{~s}, \mathrm{c} 7)$ & 100,0 \\
\hline & $44,7-40,2(d, C 6)$ & \\
\hline & $153,6-120,2(d, C 5)$ & \\
\hline & $141,1-129,6(\mathrm{~s}, \mathrm{C} 4)$ & \\
\hline & $46,9-36,9(t, C 5)$ & \\
\hline \multirow[t]{4}{*}{$10[6,10 \mathrm{OH}]$} & $74,9-73,0(\mathrm{~s}, \mathrm{C} 6)$ & 100,0 \\
\hline & $54,9-54,0(d, C 1)$ & \\
\hline & $72,572,3(\mathrm{~s}, \mathrm{C} 10)$ & \\
\hline & $68,6-68,4(t, C 15)$ & \\
\hline \multirow[t]{7}{*}{$10[4,11 \mathrm{EN} ; 13,15 \mathrm{OR}]$} & $44,0-42,7(d, C 7)$ & 100,0 \\
\hline & $66,1-65,4(t, C 13)$ & \\
\hline & $129,6-129,6(d, C 5)$ & \\
\hline & $132,8-132,8(s, C 4)$ & \\
\hline & $152,3-146,8(s, \mathrm{C} 11)$ & \\
\hline & $111,8-108,8 \quad(t, C 12)$ & \\
\hline & $69,3-67,3(t, C 15)$ & \\
\hline \multirow[t]{4}{*}{ 10[4EN;15OR] } & $51,0-36,5(d, c 6)$ & 92,9 \\
\hline & $141,3-132,1 \quad(s, C 4)$ & \\
\hline & $129,6-124,3(d, C 5)$ & \\
\hline & $68,6-67,4(t, C 15)$ & \\
\hline \multirow[t]{5}{*}{$10[4,11 \mathrm{EN} ; 15 \mathrm{OR}]$} & $141,3-132,81(s, C 4)$ & 100,0 \\
\hline & $129,6-125,5(d, C 5)$ & \\
\hline & $152,3-146,8(s, C 11)$ & \\
\hline & $111,8-108,8(\mathrm{t}, \mathrm{C} 12)$ & \\
\hline & $71,5-71,1(\mathrm{~s}, \mathrm{C} 10)$ & \\
\hline \multirow[t]{6}{*}{$10[3,10 \mathrm{OH} ; 4,11 \mathrm{EN} ; 130 \times 0 ; 130 \mathrm{Me}]$} & $131,1-128,6(d, C 5)$ & 100,0 \\
\hline & $133,8-131,1(s, C 4)$ & \\
\hline & $73,8-70,5(d, C 3)$ & \\
\hline & $125,3-124,1 \quad(t, C 11)$ & \\
\hline & $144,1-143,3 \quad(s, C 12)$ & \\
\hline & $167,8-167,1(s, C 13)$ & \\
\hline
\end{tabular}


Tabela 15. Continuação

\begin{tabular}{|c|c|c|}
\hline Subesqueleto & $\begin{array}{c}\text { Faixas de deslocamentos } \\
\text { químicos (ppm) }\end{array}$ & $\begin{array}{c}\text { Percentual de } \\
\text { Reconhecimento (\%) } \\
\end{array}$ \\
\hline & $173,3-167,5$ (s, C15) & \\
\hline \multirow[t]{3}{*}{$10[4 \mathrm{EN} ; 150 \times 0 ; 150 R]$} & $132,5-124,0 \quad(s, C 4)$ & 100,0 \\
\hline & $144,8-138,5(d, C 5)$ & \\
\hline & $125,9-119,1(d, C 5)$ & \\
\hline \multirow[t]{5}{*}{$10[4,9 \mathrm{EN}]$} & $36,5-26,5(d, C 6)$ & 100,0 \\
\hline & $39,2-36,7(d, C 1)$ & \\
\hline & $137,1-135,5$ (s, C10) & \\
\hline & $123,8-119,5(d, C 9)$ & \\
\hline & $195,1-194,6$ (d, C15) & \\
\hline \multirow[t]{3}{*}{ 10[4EN; $150 \times O]$} & $141,8-141,1 \quad(s, C 4)$ & 100,0 \\
\hline & $154,5-153,0(d, C 5)$ & \\
\hline & $53,5-48,2(d, C 4)$ & \\
\hline \multirow[t]{6}{*}{$56[8(10) E N]$} & $51,7-41,2$ (d, C9) & 100,0 \\
\hline & $49,5-41,5(d, C 5)$ & \\
\hline & $29,8-27,2$ (d, C11) & \\
\hline & $154,6-146,0(\mathrm{~s}, \mathrm{C} 8)$ & \\
\hline & $110,5-103,5(t, C 10)$ & \\
\hline & $53,5-48,0(d, C 4)$ & \\
\hline \multirow[t]{5}{*}{$56[3(14) \mathrm{EN}]$} & $53,5-41,5(d, C 5)$ & 100,0 \\
\hline & $29,7-27,2$ (d, C11) & \\
\hline & $130,1-117,0$ (d, C14) & \\
\hline & $147,3-140,1(s, C 3)$ & \\
\hline & $53,5-41,5(d, C 5)$ & \\
\hline \multirow[t]{5}{*}{$56[20 \times 0]$} & $34,9-23,7(t, C 6)$ & 100,0 \\
\hline & $29,3-27,3(d, C 11)$ & \\
\hline & $51,7-48,0(d, C 1)$ & \\
\hline & $219,3-182,1 \quad(s, C 2)$ & \\
\hline & $53,5-53,5(d, C 1)$ & \\
\hline \multirow[t]{3}{*}{$75[6,100 \mathrm{XI}]$} & $76,4-73,4 \quad(s, C 10)$ & 100,0 \\
\hline & $75,8-75,5(d, C 6)$ & \\
\hline & $67,8-67,6(d, C 5)$ & \\
\hline
\end{tabular}


Tabela 15. Continuação

\begin{tabular}{|c|c|c|}
\hline Subesqueleto & $\begin{array}{c}\text { Faixas de deslocamentos químicos } \\
\text { (ppm) }\end{array}$ & $\begin{array}{c}\text { Percentual de } \\
\text { Reconhecimento (\%) } \\
\end{array}$ \\
\hline & $44,0-35,7(d, c 7)$ & \\
\hline \multirow[t]{4}{*}{$75[10,110 \times 1]$} & $76,8-74,5(\mathrm{~s}, \mathrm{C} 11)$ & 100,0 \\
\hline & $76,4-73,3(s, \mathrm{c} 10)$ & \\
\hline & $31,1-30,6(q, C 12)$ & \\
\hline & $76,4-73,4(s, \mathrm{c} 10)$ & \\
\hline \multirow[t]{5}{*}{$75[2,8 \mathrm{OR} ; 10,110 \mathrm{OI}]$} & $76,8-74,5(s, \mathrm{C} 11)$ & 100,0 \\
\hline & $44,0-39,9(d, c 7)$ & \\
\hline & $77,0-73,6(\mathrm{~d}, \mathrm{C} 2)$ & \\
\hline & $71,8-64,3(\mathrm{~d}, \mathrm{C} 8)$ & \\
\hline & $92,5-90,4(s, C 5)$ & \\
\hline \multirow[t]{3}{*}{$75[5,110 \times 1]$} & $81,8-80,5$ (s, C11) & 100,0 \\
\hline & $46,0-45,4(d, C 7)$ & \\
\hline & $145,3-144,1 \quad(s, C 1)$ & \\
\hline \multirow[t]{4}{*}{$75[1,3,5,9 \mathrm{EN}]$} & $135,1-134,5(\mathrm{~s}, \mathrm{C} 4)$ & 100,0 \\
\hline & $136,0-132,8 \quad(s, C 5)$ & \\
\hline & $134,6-133,1 \quad(s, C 10)$ & \\
\hline & $135,8-125,5(d, C 2)$ & \\
\hline \multirow[t]{5}{*}{$75[1(10), 2,4,6,8 \mathrm{EN}]$} & $147,1-138,5(\mathrm{~s}, \mathrm{C} 5)$ & 100,0 \\
\hline & $149,3-135,6(s, c 7)$ & \\
\hline & $115,1-114,1 \quad(d, C 9)$ & \\
\hline & $139,1-135,5(s, C 10)$ & \\
\hline & $209,0-194,6(\mathrm{~s}, \mathrm{C} 8)$ & \\
\hline \multirow[t]{5}{*}{$75[7(11 \mathrm{EN}) ; 8 \mathrm{OXO}]$} & $136,6-133,5(\mathrm{~s}, \mathrm{c} 7)$ & 100,0 \\
\hline & $155,3-133,8(\mathrm{~s}, \mathrm{C} 11)$ & \\
\hline & $22,6-20,6 \quad(q, C 12)$ & \\
\hline & $23,0-22,0\left(q, c_{13}\right)$ & \\
\hline & $39,2-36,2(d, C 4)$ & \\
\hline \multirow[t]{5}{*}{$75[1(10), 11 \mathrm{EN}]$} & $49,0-46,2(d, C 5)$ & 100,0 \\
\hline & $51,2-42,7(d, C 7)$ & \\
\hline & $173,6-142,0(s, \mathrm{C} 1)$ & \\
\hline & $135,8-125,5(\mathrm{~s}, \mathrm{C} 10)$ & \\
\hline & $152,1-148,8(s, C 11)$ & \\
\hline
\end{tabular}


Tabela 15. Continuação

\begin{tabular}{|c|c|c|}
\hline Subesqueleto & $\begin{array}{l}\text { Faixas de deslocamentos } \\
\text { químicos (ppm) }\end{array}$ & $\begin{array}{c}\text { Percentual de } \\
\text { Reconhecimento (\%) }\end{array}$ \\
\hline & $39,2-36,9(d, C 4)$ & \\
\hline \multirow[t]{5}{*}{$75[1(10) E N]$} & $49,0-45,0 \quad(d, c 5)$ & 100,0 \\
\hline & $34,9-28,0(t, C 6)$ & \\
\hline & $173,6-139,8(s, C 1)$ & \\
\hline & $135,8-121,6(s, C 10)$ & \\
\hline & $49,0-48,5(d, C 5)$ & \\
\hline \multirow[t]{4}{*}{$75[1(10) E N ; 14 O \times O ; 14 O R]$} & $170,1-166,3(s, C 1)$ & 100,0 \\
\hline & $126,0-125,5(\mathrm{~s}, \mathrm{C} 10)$ & \\
\hline & $173,8-169,1(s, C 14)$ & \\
\hline & $171,0-146,1 \quad(s, C 10)$ & \\
\hline \multirow[t]{5}{*}{$75[1(10), 3 \mathrm{EN} ; 2 \mathrm{OXO}]$} & $136,0-132,8(s, C 1)$ & 100,0 \\
\hline & $197,0-196,1 \quad(s, c 2)$ & \\
\hline & $133,6-132,6(d, C 3)$ & \\
\hline & $175,5-172,0(s, C 4)$ & \\
\hline & $205,5-205,5(s, \mathrm{c} 2)$ & \\
\hline \multirow[t]{5}{*}{$75[20 \times 0 ; 3,5 \mathrm{EN}]$} & $130,8-130,0(d, C 3)$ & 100,0 \\
\hline & $174,8-169,0 \quad(s, C 4)$ & \\
\hline & $143,3-143,3(s, C 5)$ & \\
\hline & $126,6-126,3$ (d, C6) & \\
\hline & $52,7-51,7(d, C 1)$ & \\
\hline \multirow[t]{3}{*}{$75[5,80 \times 1 ; 80 H]$} & $86,9-84,6(s, C 5)$ & 100,0 \\
\hline & $103,8-97,8(s, C 8)$ & \\
\hline & $71,0-70,8(s, \mathrm{c} 5)$ & \\
\hline \multirow[t]{2}{*}{$75[5,6 \mathrm{EP}]$} & $60,5-59,7(d, c 6)$ & 100,0 \\
\hline & $60,7-60,4(s, \mathrm{C} 11)$ & \\
\hline \multirow[t]{2}{*}{$75[5,6,11,12 \mathrm{EP}]$} & $54,9-54,7$ (t, C12) & 100,0 \\
\hline & $54,0-42,7(d, c 1)$ & \\
\hline \multirow[t]{4}{*}{$75[4 \mathrm{OR} ; 10(14) \mathrm{EN}]$} & $59,5-47,2(d, C 5)$ & 100,0 \\
\hline & $81,5-80,3(s, C 4)$ & \\
\hline & $111,0-108,0(t, C 14)$ & \\
\hline & $152,5-149,1 \quad(s, C 10)$ & \\
\hline
\end{tabular}


Tabela 15. Continuação

\begin{tabular}{|c|c|c|}
\hline Subesqueleto & $\begin{array}{l}\text { Faixas de deslocamentos } \\
\text { químicos (ppm) }\end{array}$ & $\begin{array}{c}\text { Percentual de } \\
\text { Reconhecimento (\%) } \\
\end{array}$ \\
\hline & $80,8-80,3(s, C 4)$ & \\
\hline \multirow[t]{4}{*}{$75[4,6 \mathrm{OH} ; 10(14) \mathrm{EN}]$} & $72,5-71,6(d, C 6)$ & 100,0 \\
\hline & $152,5-149,1 \quad(s, C 10)$ & \\
\hline & $111,0-108,0(t, C 14)$ & \\
\hline & $75,5-73,1 \quad(s, \mathrm{C} 11)$ & \\
\hline \multirow[t]{3}{*}{$75[1(10) \mathrm{EN} ; 11 \mathrm{OH}]$} & $128,8-121,6(s, C 10)$ & 100,0 \\
\hline & $148,3-139,8(s, C 1)$ & \\
\hline & $209,6-200,5(s, C 3)$ & \\
\hline \multirow[t]{5}{*}{$75[30 \times 0 ; 4,11 E N]$} & $147,0-137,6(s, C 4)$ & 100,0 \\
\hline & $176,6-171,1 \quad(s, C 5)$ & \\
\hline & $150,3-134,8 \quad(s, C 11)$ & \\
\hline & $125,0-109,0(t, C 12)$ & \\
\hline & $36,2-34,2(t, C 2)$ & \\
\hline \multirow[t]{5}{*}{$75[1(5) \mathrm{EN}]$} & $36,7-33,4 \quad(d, C 10)$ & 100,0 \\
\hline & $46,5-43,4(d, C 4)$ & \\
\hline & $141,1-136,0(s, C 5)$ & \\
\hline & $146,8-138,8(s, C 1)$ & \\
\hline & $37,9-37,9(d, c 4)$ & \\
\hline \multirow[t]{5}{*}{ 75[1(5)EN; 20XO] } & $43,0-43,0(t, C 3)$ & 100,0 \\
\hline & $178,0-177,0(s, C 5)$ & \\
\hline & $145,3-145,1(s, C 1)$ & \\
\hline & $208,5-208,1(s, C 2)$ & \\
\hline & $142,3-142,3(s, C 1)$ & \\
\hline \multirow[t]{4}{*}{$75[1(5), 6 \mathrm{EN}]$} & $136,0-136,0(s, C 5)$ & 100,0 \\
\hline & $117,5-117,5(d, C 6)$ & \\
\hline & $150,8-150,8(\mathrm{~s}, \mathrm{C} 10)$ & \\
\hline & $35,7-30,6(\mathrm{~s}, \mathrm{C} 3)$ & \\
\hline \multirow[t]{3}{*}{74 [sem substituintes] } & $13,6-3,9(t, C 11)$ & 100,0 \\
\hline & $19,7-4,3(t, C 12)$ & \\
\hline & $55,2-37,5(\mathrm{~s}, \mathrm{C} 7)$ & \\
\hline
\end{tabular}


Tabela 15. Continuação

\begin{tabular}{|c|c|c|}
\hline Subesqueleto & $\begin{array}{c}\text { Faixas de deslocamentos } \\
\text { químicos (ppm) } \\
\end{array}$ & $\begin{array}{c}\text { Percentual de } \\
\text { Reconhecimento (\%) } \\
\end{array}$ \\
\hline & $33,7-31,7(s, \mathrm{c} 3)$ & \\
\hline \multirow[t]{4}{*}{$74[60 \times 0]$} & $11,6-6,5(t, C 12)$ & 100,0 \\
\hline & $9,0-6,5(t, C 11)$ & \\
\hline & $225,3-206,6(\mathrm{~s}, \mathrm{C} 6)$ & \\
\hline & $35,7-31,5(t, C 3)$ & \\
\hline \multirow[t]{4}{*}{$74[10 \times 0]$} & $13,6-3,9(t, C 11)$ & 100,0 \\
\hline & $11,6-4,3(t, C 12)$ & \\
\hline & $202,1-185,8(\mathrm{~s}, \mathrm{C} 1)$ & \\
\hline & $45,7-40,5(s, C 11)$ & \\
\hline \multirow[t]{4}{*}{$102[14,15 \mathrm{OXI}]$} & $54,7-47,4(s, C 6)$ & 100,0 \\
\hline & $52,4-42,0(d, C 7)$ & \\
\hline & $99,6-90,8$ (d, C14) & \\
\hline & $44,4-40,5(s, \mathrm{C} 11)$ & \\
\hline \multirow[t]{4}{*}{$102[4 \mathrm{EN}]$} & $56,2-47,4(s, C 6)$ & 100,0 \\
\hline & $146,3-120,9(d, C 4)$ & \\
\hline & $161,3-140,1(s, C 5)$ & \\
\hline & $41,0-40,0(s, C 11)$ & \\
\hline \multirow[t]{6}{*}{41 [sem substituintes] } & $54,2-37,5$ (s, C10) & 80,0 \\
\hline & $57,7-38,2(d, C 7)$ & \\
\hline & $43,7-34,2(d, C 5)$ & \\
\hline & $25,7-23,1(q, C 15)$ & \\
\hline & $27,7-25,7(q, c 14)$ & \\
\hline & $40,0-37,0(d, C 4)$ & \\
\hline \multirow[t]{3}{*}{ 125[sem substituintes] } & $48,0-44,0 \quad(\mathrm{~s}, \mathrm{C})$ & 100,0 \\
\hline & $29,0-25,0(\mathrm{~s}, \mathrm{C} 1)$ & \\
\hline & $44,0-38,5(s, C 1)$ & \\
\hline \multirow[t]{3}{*}{109 [sem substituintes] } & $65,5-63,5(d, C 11)$ & 100,0 \\
\hline & $53,2-49,2(\mathrm{~s}, \mathrm{C} 4)$ & \\
\hline & $44,0-38,5(s, C 1)$ & \\
\hline \multirow[t]{4}{*}{$109[9(12) \mathrm{EN}]$} & $65,5-63,5(d, C 11)$ & 100,0 \\
\hline & $53,2-49,2(s, C 4)$ & \\
\hline & $161,5-159,8(\mathrm{~s}, \mathrm{C} 9)$ & \\
\hline & $109,0-107,5(\mathrm{t}, \mathrm{C} 12)$ & \\
\hline
\end{tabular}


Tabela 15. Continuação

\begin{tabular}{|c|c|c|}
\hline Subesqueleto & $\begin{array}{c}\text { Faixas de deslocamentos quimicos } \\
(\mathrm{ppm})\end{array}$ & $\begin{array}{c}\text { Percentual de } \\
\text { Reconhecimento (\%) }\end{array}$ \\
\hline & $44,0-38,5(s, C 1)$ & \\
\hline \multirow[t]{5}{*}{$109[8,10 \mathrm{OH}]$} & $65,5-63,5(\mathrm{~d}, \mathrm{C} 11)$ & 100,0 \\
\hline & $53,2-49,2(\mathrm{~s}, \mathrm{C} 4)$ & \\
\hline & $73,8-72,4 \quad(d, C 8)$ & \\
\hline & $89,9-86,0 \quad(\mathrm{~s}, \mathrm{C} 10)$ & \\
\hline & $48,5-45,7(s, \mathrm{c} 3)$ & \\
\hline \multirow[t]{4}{*}{$110[6,7 \mathrm{EP}]$} & $63,7-60,9(d, c 6)$ & 100,0 \\
\hline & $76,5-73,5(\mathrm{~s}, \mathrm{C} 7)$ & \\
\hline & $53,2-43,7(s, C 11)$ & \\
\hline & $44,2-37,0 \quad(s, C 6)$ & \\
\hline \multirow[t]{4}{*}{$108[1 \mathrm{EN}]$} & $47,0-40,5(s, C 10)$ & 100,0 \\
\hline & $147,6-138,3(s, C 1)$ & \\
\hline & $130,6-127,1(s, \mathrm{c} 2)$ & \\
\hline & $59,2-54,7(\mathrm{~s}, \mathrm{C} 4)$ & \\
\hline \multirow[t]{4}{*}{119 [sem substituintes] } & $73,5-63,0 \quad(s, \mathrm{c} 8)$ & 100,0 \\
\hline & $65,1-43,0(\mathrm{~d}, \mathrm{C} 1)$ & \\
\hline & $34,9-23,2(t, C 11)$ & \\
\hline & $59,2-58,0(s, C 4)$ & \\
\hline \multirow[t]{3}{*}{$119[50 \times 0]$} & $68,3-63,0(s, C 8)$ & 100,0 \\
\hline & $213,8-208,1 \quad(s, C 5)$ & \\
\hline & $59,2-57,5(\mathrm{~s}, \mathrm{C} 4)$ & \\
\hline \multirow[t]{3}{*}{$119[130 \times 0 ; 130 R]$} & $73,5-63,0 \quad(s, C 8)$ & 100,0 \\
\hline & $164,8-159,1 \quad(s, C 13)$ & \\
\hline & $59,2-54,7(s, C 4)$ & \\
\hline \multirow[t]{4}{*}{$119[6 \mathrm{EN}]$} & $73,5-67,3(s, C 8)$ & 100,0 \\
\hline & $188,6-157,3(\mathrm{~s}, \mathrm{C} 7)$ & \\
\hline & $133,3-127,0(s, C 6)$ & \\
\hline & $57,7-56,5(\mathrm{~s}, \mathrm{C} 4)$ & \\
\hline \multirow[t]{3}{*}{$119[5 E N]$} & $66,1-64,4(s, C 8)$ & 100,0 \\
\hline & $133,6-107,0(d, C 5)$ & \\
\hline & $142,5-133,6(s, c 6)$ & \\
\hline
\end{tabular}


Tabela 15. Continuação

\begin{tabular}{|c|c|c|}
\hline Subesqueleto & $\begin{array}{c}\text { Faixas de deslocamentos } \\
\text { químicos (ppm) }\end{array}$ & $\begin{array}{c}\text { Percentual de } \\
\text { Reconhecimento (\%) }\end{array}$ \\
\hline & $28,2-25,6(t, C 2)$ & \\
\hline \multirow[t]{4}{*}{78 [sem substituintes] } & $70,0-61,7(s, C 1)$ & 100,0 \\
\hline & $43,5-41,0(s, \mathrm{C} 11)$ & \\
\hline & $63,0-47,2(d, c 7)$ & \\
\hline & $66,3-51,9(\mathrm{~s}, \mathrm{C} 1)$ & \\
\hline \multirow[t]{3}{*}{121 [sem substituintes] } & $61,5-56,0(d, C 5)$ & 66,7 \\
\hline & $48,0-42,9(\mathrm{~s}, \mathrm{C} 6)$ & \\
\hline & $54,0-52,5(\mathrm{~s}, \mathrm{C} 1)$ & \\
\hline \multirow[t]{3}{*}{$121[8 \mathrm{OH}]$} & $44,0-42,9(\mathrm{~s}, \mathrm{C} 6)$ & 100,0 \\
\hline & $75,1-74,0(\mathrm{~s}, \mathrm{C} 8)$ & \\
\hline & $66,3-51,9(\mathrm{~s}, \mathrm{C} 1)$ & \\
\hline \multirow[t]{4}{*}{$121[8 \mathrm{EN}]$} & $48,0-44,0 \quad(s, C 6)$ & 100,0 \\
\hline & $165,6-140,0(\mathrm{~s}, \mathrm{C} 8)$ & \\
\hline & $125,8-119,0(d, C 9)$ & \\
\hline & $68,5-45,9(d, C 2)$ & \\
\hline \multirow[t]{4}{*}{ 92[sem substituintes] } & $65,0-39,5$ (d, c11) & 100,0 \\
\hline & $40,9-37,0(s, \mathrm{C} 10)$ & \\
\hline & $40,4-35,2(s, c 6)$ & \\
\hline & $44,7-22,8(d, C 6)$ & \\
\hline \multirow[t]{5}{*}{35 [sem substituintes] } & $39,5-16,7(d, C 7)$ & 100,0 \\
\hline & $35,2-17,8(s, C 11)$ & \\
\hline & $40,4-31,1(d, C 4)$ & \\
\hline & $39,5-36,2(s, \mathrm{C} 5)$ & \\
\hline & $38,9-36,2(s, C 5)$ & \\
\hline \multirow[t]{5}{*}{$35[1(10) \mathrm{EN}]$} & $44,7-29,8 \quad(d, C 6)$ & 100,0 \\
\hline & $30,7-16,7(d, C 7)$ & \\
\hline & $35,2-18,7(s, C 11)$ & \\
\hline & $136,3-116,1 \quad(d, C 1)$ & \\
\hline & $174,3-142,1(\mathrm{~s}, \mathrm{C} 10)$ & \\
\hline
\end{tabular}


Tabela 15. Continuação

\begin{tabular}{|c|c|c|}
\hline Subesqueleto & $\begin{array}{l}\text { Faixas de deslocamentos químicos } \\
\qquad(\mathrm{ppm})\end{array}$ & $\begin{array}{c}\text { Percentual de } \\
\text { Reconhecimento }(\%) \\
\end{array}$ \\
\hline & $36,7-36,2(s, \mathrm{c} 5)$ & \\
\hline \multirow[t]{5}{*}{$35[120 \times 0]$} & $44,7-32,4(d, C 6)$ & 100,0 \\
\hline & $30,7-20,7(d, C 7)$ & \\
\hline & $35,2-34,5(\mathrm{~s}, \mathrm{C} 11)$ & \\
\hline & $205,0-202,8(d, C 12)$ & \\
\hline & $39,5-36,2(s, c 5)$ & \\
\hline \multirow[t]{6}{*}{$35[9 \mathrm{EN}]$} & $40,4-32,0(d, C 6)$ & 100,0 \\
\hline & $36,5-20,7(d, C 7)$ & \\
\hline & $34,5-17,8$ (s, C11) & \\
\hline & $167,0-141,0(s, C 10)$ & \\
\hline & $127,3-117,4(d, C 9)$ & \\
\hline & $66,0-58,5(\mathrm{~d}, \mathrm{C} 1)$ & \\
\hline \multirow[t]{6}{*}{$35[1,10 \mathrm{EP}]$} & $64,6-63,7(\mathrm{~s}, \mathrm{C} 10)$ & 100,0 \\
\hline & $36,5-36,2(s, C 5)$ & \\
\hline & $44,0-22,8(d, C 6)$ & \\
\hline & $39,5-35,4(d, C 7)$ & \\
\hline & $31,0-21,0(s, C 11)$ & \\
\hline & $27,7-18,8 \quad(\mathrm{~s}, \mathrm{C} 11)$ & \\
\hline \multirow[t]{7}{*}{$35[1(10) E N ; 20 \times O]$} & $174,3-162,8(s, C 10)$ & 100,0 \\
\hline & $129,6-122,5(d, C 1)$ & \\
\hline & $199,5-199,1 \quad(s, C 2)$ & \\
\hline & $38,9-36,2(s, C 5)$ & \\
\hline & $34,0-32,0(d, c 6)$ & \\
\hline & $26,1-16,7(d, C 7)$ & \\
\hline & $39,5-36,5(\mathrm{~s}, \mathrm{C} 5)$ & \\
\hline \multirow[t]{4}{*}{$35[80 \times 0]$} & $44,0-39,2(d, C 6)$ & 100,0 \\
\hline & $39,5-35,5(\mathrm{~d}, \mathrm{C} 7)$ & \\
\hline & $31,0-24,2$ (s, C11) & \\
\hline & $197,1-188,8(\mathrm{~s}, \mathrm{C} 8)$ & \\
\hline
\end{tabular}


Tabela 15. Continuação

\begin{tabular}{|c|c|c|}
\hline Subesqueleto & $\begin{array}{c}\text { Faixas de deslocamentos químicos } \\
(\mathrm{ppm})\end{array}$ & $\begin{array}{c}\text { Percentual de } \\
\text { Reconhecimento (\%) }\end{array}$ \\
\hline & $42,4-41,7 \quad(d, C 9)$ & \\
\hline \multirow[t]{5}{*}{$48[$ sem substituintes] } & $20,3-19,7 \quad(s, C 10)$ & 80,0 \\
\hline & $19,5-19,0(t, C 11)$ & \\
\hline & $45,0-42,0(\mathrm{~s}, \mathrm{C} 7)$ & \\
\hline & $23,2-22,7(q, C 14)$ & \\
\hline & $46,0-42,0(\mathrm{~s}, \mathrm{C5})$ & \\
\hline \multirow[t]{4}{*}{$76[1(10) E N ; 2 O X O]$} & $209,0-207,0 \quad(s, \mathrm{C} 2)$ & 100,0 \\
\hline & $155,0-153,0(\mathrm{~s}, \mathrm{C} 1)$ & \\
\hline & $139,0-136,0(s, C 10)$ & \\
\hline & $134,1-119,9(\mathrm{~s}, \mathrm{C} 7)$ & \\
\hline \multirow[t]{4}{*}{$21[8,12$ FURANO $]$} & $154,3-136,3(s, C 8)$ & 100,0 \\
\hline & $121,9-116,1$ (s, C11) & \\
\hline & $147,0-140,6(d, C 12)$ & \\
\hline & $58,0-56,2(5, C 4)$ & \\
\hline \multirow[t]{3}{*}{120 [sem substituintes] } & $53,0-52,0(d, C 7)$ & 100,0 \\
\hline & $51,5-47,4(\mathrm{~s}, \mathrm{C} 6)$ & \\
\hline & $23,2-19,0(d, C 6)$ & \\
\hline \multirow[t]{4}{*}{36 [sem substituintes] } & $29,6-19,3(d, c 7)$ & 100,0 \\
\hline & $36,4-33,2(s, \mathrm{C} 10)$ & \\
\hline & $19,0-17,7$ (s, c11) & \\
\hline & $43,2-35,2(s, C 7)$ & \\
\hline \multirow[t]{3}{*}{$117[10 \times 0 ; 2 E N]$} & $46,0-37,5(\mathrm{~s}, \mathrm{C} 11)$ & 100,0 \\
\hline & $196,6-192,1$ (d, C1) & \\
\hline & $52,2-39,7(s, C 7)$ & \\
\hline \multirow[t]{4}{*}{$117[2(4) E N]$} & $47,5-38,5$ (s, C11) & 100,0 \\
\hline & $136,8-123,0 \quad(s, C 2)$ & \\
\hline & $164,5-127,8(\mathrm{~s}, \mathrm{C} 4)$ & \\
\hline & $43,2-34,0(s, C 7)$ & \\
\hline \multirow[t]{3}{*}{$117[2 \mathrm{EN}]$} & $46,0-37,5(s, C 11)$ & 100,0 \\
\hline & $158,8-131,3(s, C 2)$ & \\
\hline & $159,1-132,6(d, C 3)$ & \\
\hline
\end{tabular}


Tabela 15. Continuação

\begin{tabular}{|c|c|c|}
\hline Subesqueleto & $\begin{array}{l}\text { Faixas de desiocamentos químicos } \\
(\mathrm{ppm})\end{array}$ & $\begin{array}{c}\text { Percentual de } \\
\text { Reconhecimento (\%) } \\
\end{array}$ \\
\hline & $43,2-34,0(s, C 7)$ & \\
\hline \multirow[t]{3}{*}{$117[4,5 \mathrm{OR}]$} & $46,0-37,5$ (s, C11) & 100,0 \\
\hline & $82,0-74,3(\mathrm{~s}, \mathrm{C} 4)$ & \\
\hline & $78,6-72,5(\mathrm{~d}, \mathrm{C} 5)$ & \\
\hline \multirow{4}{*}{$117[1,3 \mathrm{OR}]$} & $52,2-37,0(\mathrm{~s}, \mathrm{C} 7)$ & \\
\hline & $40,4-38,5(\mathrm{~s}, \mathrm{C} 11)$ & 100,0 \\
\hline & $67,9-58,5(t, C 1)$ & \\
\hline & $75,6-69,3(d, c 3)$ & \\
\hline \multirow{3}{*}{$117[1 \mathrm{OR}]$} & $67,9-58,5(\mathrm{t}, \mathrm{C} 1)$ & \\
\hline & $52,2-34,0(\mathrm{~s}, \mathrm{c} 7)$ & 91,0 \\
\hline & $43,2-38,5(s, c 11)$ & \\
\hline \multirow{5}{*}{$117[1,4,5 \mathrm{OR}]$} & $38,9-34,0 \quad(s, C 7)$ & \\
\hline & $43,2-38,7(s, C 11)$ & 100,0 \\
\hline & $67,5-63,0(t, C 1)$ & \\
\hline & $82,0-76,3(\mathrm{~s}, \mathrm{C} 4)$ & \\
\hline & $78,6-72,5(d, c 5)$ & \\
\hline \multirow{5}{*}{$117[4,5,10 \mathrm{OR}]$} & $37,0-35,2(s, C 7)$ & \\
\hline & $46,0-40,7(\mathrm{~s}, \mathrm{C} 11)$ & 100,0 \\
\hline & $77,3-74,3(s, C 4)$ & \\
\hline & $77,1-72,5(d, C 5)$ & \\
\hline & $83,0-79,5(d, c 10)$ & \\
\hline \multirow{3}{*}{$117[10 \mathrm{OR}]$} & $49,7-35,2(s, C 7)$ & \\
\hline & $47,5-40,7(s, C 11)$ & 92,0 \\
\hline & $83,0-79,5(\mathrm{~d}, \mathrm{c} 10)$ & \\
\hline \multirow{6}{*}{$117[4,5,10,13 \mathrm{OR}]$} & $77,3-75,1 \quad(s, \mathrm{C} 4)$ & \\
\hline & $75,4-72,5(d, C 5)$ & 100,0 \\
\hline & $83,0-79,5(d, C 10)$ & \\
\hline & $77,5-74,8(s, \mathrm{C} 13)$ & \\
\hline & $37,0-35,7(s, c 7)$ & \\
\hline & $46,0-40,7(s, C 11)$ & \\
\hline
\end{tabular}


Tabela 15. Continuação

\begin{tabular}{|c|c|c|}
\hline Subesqueleto & $\begin{array}{c}\text { Faixas de deslocamentos químicos } \\
(\mathrm{ppm})\end{array}$ & $\begin{array}{c}\text { Percentual de } \\
\text { Reconhecimento (\%) } \\
\end{array}$ \\
\hline & $50,0-44,4(d, C 4)$ & \\
\hline \multirow[t]{4}{*}{$93[10 \times 0]}$, & $41,9-33,7(s, c 6)$ & 100,0 \\
\hline & $64,3-44,0(s, C 10)$ & \\
\hline & $212,1-200,8(s, C 1)$ & \\
\hline & $41,9-33,7(s, \mathrm{C} 6)$ & \\
\hline \multirow[t]{5}{*}{$93[10 \times O ; 2 E N]$} & $64,3-54,5(s, C 10)$ & 100,0 \\
\hline & $172,3-169,3 \quad(s, C 3)$ & \\
\hline & $122,9-122,0 \quad(d, C 2)$ & \\
\hline & $204,6-200,8(s, C 1)$ & \\
\hline & $200,6-197,8(s, C 2)$ & \\
\hline \multirow[t]{5}{*}{$93[20 \times O ; 3(12) E N]$} & $150,5-147,3(\mathrm{~s}, \mathrm{C} 3)$ & 100,0 \\
\hline & $118,1-115,4$ (t, $C 12)$ & \\
\hline & $36,5-33,0(s, C 6)$ & \\
\hline & $44,0-42,4(\mathrm{~s}, \mathrm{C} 10)$ & \\
\hline & $76,5-74,8$ (d, c9) & \\
\hline \multirow[t]{4}{*}{$93[1,90 R ; 2 \mathrm{EN}]$} & $150,5-148,1 \quad(s, C 3)$ & 100,0 \\
\hline & $120,8-117,5(d, C 2)$ & \\
\hline & $75,6-74,0(d, c 1)$ & \\
\hline & $36,5-35,0(\mathrm{~s}, \mathrm{C} 6)$ & \\
\hline \multirow[t]{5}{*}{$93[7,8,90 R]$} & $55,4-44,0(\mathrm{~s}, \mathrm{C} 10)$ & 100,0 \\
\hline & $75,0-70,5(d, C 1)$ & \\
\hline & $71,5-68,6(d, C 8)$ & \\
\hline & $77,1-73,6(d, C 9)$ & \\
\hline & $74,8-64,8 \quad(d, C 7)$ & \\
\hline \multirow[t]{5}{*}{$93[7,90 R]$} & $36,7-32,0(t, C 8)$ & 94,0 \\
\hline & $76,5-73,0(d, C 9)$ & \\
\hline & $41,9-35,2(s, C 6)$ & \\
\hline & $57,2-45,5(\mathrm{~s}, \mathrm{C} 10)$ & \\
\hline & $48,4-39,5(\mathrm{~s}, \mathrm{C} 10)$ & \\
\hline \multirow[t]{4}{*}{$128[1,3 \mathrm{EN}]$} & $114,8-109,8(t, C 2)$ & 100,0 \\
\hline & $150,3-136,8(d, C 1)$ & \\
\hline & $130,1-110,6(t, C 3)$ & \\
\hline & $148,0-132,6(s, C 4)$ & \\
\hline
\end{tabular}


Tabela 15. Continuação

\begin{tabular}{|c|c|c|}
\hline Subesqueleto & $\begin{array}{l}\text { Faixas de deslocamentos } \\
\text { químicos (ppm) }\end{array}$ & $\begin{array}{c}\text { Percentual de } \\
\text { Reconhecimento (\%) } \\
\end{array}$ \\
\hline & $78,5-77,4(d, c 6)$ & \\
\hline \multirow[t]{4}{*}{$128[6,12 \mathrm{OXI} ; 12 \mathrm{OR}]$} & $54,0-53,4(d, C 7)$ & 100,0 \\
\hline & $43,0-41,0 \quad(d, C 11)$ & \\
\hline & $109,6-98,5(d, C 12)$ & \\
\hline & $150,3-136,8(d, C 1)$ & 87,5 \\
\hline \multirow[t]{5}{*}{$128[1,3,11 E N]$} & $115,0-110,8(t, C 3)$ & \\
\hline & $147,6-139,3(s, C 4)$ & \\
\hline & $150,3-137,8$ (s, C11) & \\
\hline & $128,3-108,3(t, C 12)$ & \\
\hline & $46,2-44,9(s, C 7)$ & 100,0 \\
\hline \multirow[t]{4}{*}{126 [sem substituintes] } & $51,7-46,9(d, C 2)$ & \\
\hline & $37,0-34,2(t, C 3)$ & \\
\hline & $46,5-44,9(d, C 4)$ & \\
\hline & $40,7-29,6(s, C 4)$ & 94,0 \\
\hline \multirow[t]{4}{*}{ 13[7EN] } & $42,2-32,9(\mathrm{~s}, \mathrm{C} 10)$ & \\
\hline & $157,1-117,0(d, C 7)$ & \\
\hline & $143,8-127,1(s, C 8)$ & \\
\hline & $54,2-33,4(s, c 4)$ & 100,0 \\
\hline \multirow[t]{4}{*}{$13[8(12) \mathrm{EN}]$} & $39,7-37,2$ (s, C10) & \\
\hline & $148,3-146,1(s, C 8)$ & \\
\hline & $110,9-106,0 \quad(t, C 12)$ & \\
\hline & $54,2-33,4(s, C 4)$ & 100,0 \\
\hline \multirow[t]{5}{*}{ 13[8(12)EN; 11OR] } & $39,7-37,2(s, \mathrm{C} 10)$ & \\
\hline & $148,3-146,1 \quad(s, C 8)$ & \\
\hline & $110,9-106,0 \quad(t, C 12)$ & \\
\hline & $73,6-58,0(\mathrm{t}, \mathrm{C} 11)$ & \\
\hline & $34,9-33,0 \quad(s, C 4)$ & 100,0 \\
\hline \multirow[t]{4}{*}{ 13[7EN; $9 \mathrm{OH}]$} & $41,7-39,9$ (s, C10) & \\
\hline & $82,0-74,6(s, C 9)$ & \\
\hline & $143,8-133,8(s, C 8)$ & \\
\hline & $157,1-121,1 \quad(d, C 7)$ & \\
\hline
\end{tabular}


Tabela 15. Continuação

\begin{tabular}{|c|c|c|}
\hline Subesqueleto & $\begin{array}{c}\text { Faixas de deslocamentos químicos } \\
(\mathrm{ppm})\end{array}$ & $\begin{array}{c}\text { Percentual de } \\
\text { Reconhecimento (\%) } \\
\end{array}$ \\
\hline \multirow{6}{*}{$13[7 \mathrm{EN} ; 11,120 \times \mathrm{O}]$} & $37,7-29,6 \quad(s, C 4)$ & 100,0 \\
\hline & $42,2-36,2(s, C 10)$ & \\
\hline & $157,1-148,5(d, C 7)$ & \\
\hline & $141,3-137,3(s, C 8)$ & \\
\hline & $202,3-192,5$ (d, C11) & \\
\hline & $202,0-192,5(d, C 12)$ & \\
\hline \multirow{5}{*}{ 13[7EN; 3OR] } & $40,7-34,0(s, C 4)$ & 100,0 \\
\hline & $80,5-75,6(d, c 3)$ & \\
\hline & $37,5-32,9(\mathrm{~s}, \mathrm{C} 10)$ & \\
\hline & $155,3-120,9(d, C 7)$ & \\
\hline & $138,3-127,1(\mathrm{~s}, \mathrm{C} 8)$ & \\
\hline \multirow{5}{*}{ 13[3OR; 8(12)EN] } & $79,0-75,0 \quad(d, c 3)$ & 90,0 \\
\hline & $54,2-36,5(s, C 4)$ & \\
\hline & $39,7-37,2\left(s, C_{10}\right)$ & \\
\hline & $148,3-146,1 \quad(s, C 8)$ & \\
\hline & $110,9-107,6(t, C 12)$ & \\
\hline \multirow{5}{*}{$13[30 R]$} & $80,5-75,0(\mathrm{~d}, \mathrm{C} 3)$ & \\
\hline & $54,2-34,0(s, C 4)$ & 100,0 \\
\hline & $64,5-37,0(d, C 5)$ & \\
\hline & $39,7-33,2(s, \mathrm{C} 10)$ & \\
\hline & $60,7-50,5(\mathrm{~d}, \mathrm{C} 9)$ & \\
\hline \multirow{6}{*}{$87[100 R]$} & $30,1-22,3(d, C 6)$ & \\
\hline & $31,0-22,6(d, c 7)$ & 100,0 \\
\hline & $26,3-18,3(s, \mathrm{C} 11)$ & \\
\hline & $48,2-37,7(d, C 5)$ & \\
\hline & $58,5-52,5(d, C 1)$ & \\
\hline & $82,9-71,8(\mathrm{~s}, \mathrm{C} 10)$ & \\
\hline \multirow{2}{*}{$87[10,14 \mathrm{OH}]$} & $76,4-75,0 \quad(s, \mathrm{C} 10)$ & \\
\hline & $72,1-70,8(\mathrm{t}, \mathrm{C} 14)$ & 100,0 \\
\hline
\end{tabular}


Tabela 15. Continuação

\begin{tabular}{|c|c|c|}
\hline Subesqueleto & $\begin{array}{c}\text { Faixas de deslocamentos químicos } \\
(\mathrm{ppm})\end{array}$ & $\begin{array}{c}\text { Percentual de } \\
\text { Reconhecimento (\%) }\end{array}$ \\
\hline & $31,3-21,8(d, C 6)$ & \\
\hline \multirow[t]{5}{*}{$87[1,5,6,7$ sem substituintes $]$} & $31,7-22,6(d, C 7)$ & 90,7 \\
\hline & $26,3-17,2\left(s, c_{11}\right)$ & \\
\hline & $56,4-37,7(d, C 5)$ & \\
\hline & $58,5-47,0(d, C 1)$ & \\
\hline & $29,5-21,8(d, C 6)$ & \\
\hline \multirow[t]{6}{*}{$87[1,4,5,6,7$ sem substituintes $]$} & $31,0-22,6(d, c 7)$ & 100,0 \\
\hline & $26,3-17,2\left(s, c_{11}\right)$ & \\
\hline & $44,0-37,7(d, C 5)$ & \\
\hline & $58,5-49,9(d, C 1)$ & \\
\hline & $39,5-34,0(d, c 4)$ & \\
\hline & $31,3-23,6(d, C 6)$ & \\
\hline \multirow[t]{5}{*}{$87[10(14) E N]$} & $31,7-23,7(d, c 7)$ & 100,0 \\
\hline & $20,7-17,2(s, C 11)$ & \\
\hline & $157,5-147,6(s, C 10)$ & \\
\hline & $110,3-101,9(t, C 14)$ & \\
\hline & $26,7-21,8(d, C 6)$ & \\
\hline \multirow[t]{5}{*}{$87[9 E N]$} & $29,0-26,3 \quad(d, C 7)$ & 100,0 \\
\hline & $18,818,0(s, \mathrm{c} 11)$ & \\
\hline & $122,4-121,8(d, C 9)$ & \\
\hline & $139,3-138,8(s, C 10)$ & \\
\hline & $30,1-24,7(d, C 6)$ & \\
\hline \multirow[t]{5}{*}{$87[4,10 \mathrm{OH}]$} & $28,7-26,2(d, c 7)$ & 100,0 \\
\hline & $20,7-18,6(s, C 11)$ & \\
\hline & $82,0-80,0(s, C 4)$ & \\
\hline & $75,3-71,8(\mathrm{~s}, \mathrm{C} 10)$ & \\
\hline & $30,7-27,1 \quad(d, C 6)$ & \\
\hline \multirow[t]{4}{*}{$87[1(10) E N]$} & $30,8-25,7(d, c 7)$ & 100,0 \\
\hline & $25,0-18,6(s, \mathrm{C} 11)$ & \\
\hline & $150,1-139,8(s, C 1)$ & \\
\hline & $136,3-124,3(s, C 10)$ & \\
\hline
\end{tabular}


Tabela 15. Continuação

\begin{tabular}{|c|c|c|}
\hline Subesqueleto & $\begin{array}{l}\text { Faixas de deslocamentos químicos } \\
(\mathrm{ppm})\end{array}$ & $\begin{array}{c}\text { Percentual de } \\
\text { Reconhecimento (\%) }\end{array}$ \\
\hline & $28,3-27,1 \quad(d, C 6)$ & \\
\hline \multirow[t]{6}{*}{$87[30 \times 0 ; 4 E N]$} & $31,6-30,8(d, c 7)$ & 100,0 \\
\hline & $25,8-25,0(s, \mathrm{C} 11)$ & \\
\hline & $208,1-202,5(\mathrm{~s}, \mathrm{C} 3)$ & \\
\hline & $149,3-140,1 \quad(s, C 4)$ & \\
\hline & $176,5-166,1 \quad(s, C 5)$ & \\
\hline & $26,0-24,0(\mathrm{~d}, \mathrm{C} 6)$ & \\
\hline \multirow[t]{7}{*}{$87[1(5), 3 E N]$} & $36,0-34,0(d, c 7)$ & 100,0 \\
\hline & $22,0-19,0(s, C 11)$ & \\
\hline & $125,0-122,0(\mathrm{~d}, \mathrm{C} 3)$ & \\
\hline & $145,0-137,0 \quad(s, C 4)$ & \\
\hline & $146,0-137,0(s, C 5)$ & \\
\hline & $147,0-144,0(s, C 1)$ & \\
\hline & $35,0-32,0(s, C 1)$ & \\
\hline \multirow[t]{3}{*}{$34[$ sem substituinte] } & $42,0-36,0(d, C 5)$ & 100,0 \\
\hline & $27,0-21,0(d, c 6)$ & \\
\hline & $37,9-33,4(s, c 1)$ & \\
\hline \multirow[t]{5}{*}{$90[$ sem substituintes] } & $31,5-30,7(d, C 5)$ & 100,0 \\
\hline & $26,1-20,2(d, C 6)$ & \\
\hline & $50,2-49,4(d, C 7)$ & \\
\hline & $39,5-35,9(d, C 10)$ & \\
\hline & $57,0-55,0 \quad(d, C 10)$ & \\
\hline \multirow[t]{3}{*}{111 [sem substituintes] } & $54,5-45,2(d, C 1)$ & 100,0 \\
\hline & $50,4-45,7(\mathrm{~s}, \mathrm{c} 7)$ & \\
\hline & $49,0-45,0 \quad(s, C 7)$ & \\
\hline \multirow[t]{4}{*}{$111[5 \mathrm{OXO} ; 3 \mathrm{EN}]$} & $182,0-178,0 \quad(s, C 3)$ & 100,0 \\
\hline & $137,0-131,0(d, C 4)$ & \\
\hline & $213,0-210,0(s, C 5)$ & \\
\hline & $48,0-44,0(d, c 7)$ & \\
\hline \multirow[t]{3}{*}{$111[3,15 \mathrm{EP} ; 6 \mathrm{OR}]$} & $52,0-48,0(\mathrm{~s}, \mathrm{C} 1)$ & 100,0 \\
\hline & $67,0-63,0(\mathrm{~s}, \mathrm{C} 3)$ & \\
\hline & $59,0-55,0(t, C 15)$ & \\
\hline
\end{tabular}


Tabela 15. Continuação

\begin{tabular}{|c|c|c|}
\hline Subesqueleto & $\begin{array}{c}\text { Faixas de deslocamentos } \\
\text { químicos (ppm) }\end{array}$ & $\begin{array}{c}\text { Percentual de } \\
\text { Reconhecimento (\%) }\end{array}$ \\
\hline & $25,8-22,6(s, C 1)$ & \\
\hline \multirow[t]{4}{*}{116 [sem substituintes] } & $31,7-26,3(d, C 11)$ & 100,0 \\
\hline & $32,2-27,1(d, C 4)$ & \\
\hline & $45,7-42,5(d, C 5)$ & \\
\hline & $121,6-110,3(t, C 1)$ & \\
\hline \multirow[t]{6}{*}{$134[1,6,10 \mathrm{EN}]$} & $146,1-134,6(d, C 2)$ & 92,0 \\
\hline & $131,1-122,0(d, C 6)$ & \\
\hline & $139,8-129,3(\mathrm{~s}, \mathrm{C} 7)$ & \\
\hline & $129,3-119,0(d, C 10)$ & \\
\hline & $155,6-129,8(\mathrm{~s}, \mathrm{C} 11)$ & \\
\hline & $74,3-72,0 \quad(s, \mathrm{C} 3)$ & \\
\hline \multirow[t]{4}{*}{$134[1 \mathrm{EN} ; 3 \mathrm{OH}]$} & $47,5-25,7(t, C 4)$ & 96,7 \\
\hline & $112,6-111,0(t, C 1)$ & \\
\hline & $147,1-143,6(d, C 2)$ & \\
\hline & $73,6-73,0(\mathrm{~s}, \mathrm{C})$ & \\
\hline \multirow[t]{4}{*}{ 134[1EN; $3,70 \times 1]$} & $82,4-77,0(s, C 3)$ & 100,0 \\
\hline & $112,5-110,0(t, C 1)$ & \\
\hline & $147,3-144,6(d, C 2)$ & \\
\hline & $84,4-80,3(d, C 6)$ & \\
\hline \multirow[t]{4}{*}{$134[1 \mathrm{EN} ; 3,6 \mathrm{OXI}]$} & $84,5-82,4(s, C 3)$ & 100,0 \\
\hline & $144,3-143,8(d, C 2)$ & \\
\hline & $111,6-111,0(t, C 1)$ & \\
\hline & $121,6-110,0(t, C 1)$ & \\
\hline \multirow[t]{2}{*}{$134[1 \mathrm{EN}]$} & $147,3-134,6(d, C 2)$ & 100,0 \\
\hline & $106,6-106,0(d, C 10)$ & \\
\hline \multirow[t]{4}{*}{ 134[9,12FURANO $]$} & $119,9-119,1 \quad(s, C 11)$ & 100,0 \\
\hline & $156,8-155,0 \quad(s, \mathrm{C} 9)$ & \\
\hline & $141,3-129,6(d, C 12)$ & \\
\hline & $111,9-110,9(d, C 2)$ & \\
\hline \multirow[t]{3}{*}{ 134[1,5FURANO] } & $124,8-120,6(s, C 3)$ & 100,0 \\
\hline & $142,6-140,5(d, C 1)$ & \\
\hline & $142,8-133,8(d, c 15)$ & \\
\hline
\end{tabular}


Tabela 15. Continuação

\begin{tabular}{|c|c|c|}
\hline Subesqueleto & $\begin{array}{c}\text { Faixas de deslocamentos químicos } \\
(\mathrm{ppm})\end{array}$ & $\begin{array}{c}\text { Percentual de } \\
\text { Reconhecimento (\%) } \\
\end{array}$ \\
\hline \multirow{6}{*}{$134[2,6,10 \mathrm{EN}]$} & $127,0-119,6(d, C 2)$ & \multirow{6}{*}{100,0} \\
\hline & $142,3-136,1 \quad(s, C 3)$ & \\
\hline & $128,6-122,1 \quad(d, C 6)$ & \\
\hline & $163,3-130,0(\mathrm{~s}, \mathrm{C} 7)$ & \\
\hline & $131,3-123,0(d, C 10)$ & \\
\hline & $155,5-130,8(\mathrm{~s}, \mathrm{C} 11)$ & \\
\hline \multirow{4}{*}{$134[1 \mathrm{EN} ; 5,90 \mathrm{XI}]$} & $70,9-65,8(d, c 5)$ & \multirow{4}{*}{100,0} \\
\hline & $72,3-69,8(\mathrm{~d}, \mathrm{C9})$ & \\
\hline & $112,6-111,0(t, C 1)$ & \\
\hline & $146,1-144,5(d, C 2)$ & \\
\hline \multirow{4}{*}{$134[1,6 \mathrm{EN}]$} & $121,6-110,3(t, C 1)$ & \multirow{4}{*}{85,0} \\
\hline & $147,1-134,6(d, C 2)$ & \\
\hline & $131,1-122,0(d, C 6)$ & \\
\hline & $139,8-129,3(\mathrm{~s}, \mathrm{c} 7)$ & \\
\hline \multirow{5}{*}{$134[1 \mathrm{OR} ; 2,6 \mathrm{EN}]$} & $70,3-58,5(t, \mathrm{C} 1)$ & \multirow{5}{*}{96,3} \\
\hline & $126,3-118,1 \quad(d, C 2)$ & \\
\hline & $142,3-136,6(s, C 3)$ & \\
\hline & $128,6-123,8(d, C 6)$ & \\
\hline & $136,5-130,0(\mathrm{~s}, \mathrm{C} 7)$ & \\
\hline \multirow{3}{*}{$134[90 \times O ; 10 E N]$} & $200,3-198,8$ (s, C9) & \multirow{3}{*}{100,0} \\
\hline & $129,1-123,0(d, C 10)$ & \\
\hline & $158,3-155,3(s, C 11)$ & \\
\hline \multirow{5}{*}{38 [sem substituintes] } & $33,9-31,2(s, C 4)$ & \multirow{5}{*}{100,0} \\
\hline & $17,2-4,5 \quad(t, C 11)$ & \\
\hline & $41,4-34,7(s, C 10)$ & \\
\hline & $39,0-33,2(s, C 5)$ & \\
\hline & $27,3-23,6(d, C 6)$ & \\
\hline \multirow{3}{*}{ 58[sem substituintes] } & $38,0-34,0(s, C 4)$ & \multirow{3}{*}{100,0} \\
\hline & $50,0-43,0(s, \mathrm{C} 5)$ & \\
\hline & $55,0-47,0(\mathrm{~s}, \mathrm{C} 9)$ & \\
\hline
\end{tabular}


Tabela 15. Continuação

\begin{tabular}{|c|c|c|}
\hline Subesqueleto & $\begin{array}{l}\text { Faixas de deslocamentos químicos } \\
(\mathrm{ppm})\end{array}$ & $\begin{array}{c}\text { Percentual de } \\
\text { Reconhecimento (\%) }\end{array}$ \\
\hline & $44,5-34,7(s, C 4)$ & \\
\hline \multirow[t]{4}{*}{114 [sem substituintes] } & $47,5-37,4(s, \mathrm{C} 1)$ & 100,0 \\
\hline & $36,7-30,2(t, C 12)$ & \\
\hline & $38,2-34,4(s, C 8)$ & \\
\hline & $39,0-32,0(\mathrm{~s}, \mathrm{C} 1)$ & \\
\hline \multirow[t]{5}{*}{$90[$ sem substituintes] } & $33,0-30,0(d, C 5)$ & 100,0 \\
\hline & $27,0-20,0(d, C 6)$ & \\
\hline & $51,0-48,0(d, C 7)$ & \\
\hline & $39,5-35,9$ (d, c10) & \\
\hline & $59,0-55,0(\mathrm{~s}, \mathrm{C} 4)$ & \\
\hline \multirow[t]{3}{*}{ 115[sem substituintes] } & $66,0-57,0(\mathrm{~s}, \mathrm{C} 8)$ & 100,0 \\
\hline & $67,0-49,0(\mathrm{~s}, \mathrm{C} 7)$ & \\
\hline & $139,3-130,0(d, C 1)$ & \\
\hline \multirow[t]{5}{*}{$22[1(10) E N ; 9 O X O]$} & $155,6-140,0\left(s, C_{10}\right)$ & 100,0 \\
\hline & $200,6-187,8 \quad(s, C 9)$ & \\
\hline & $44,7-39,0(s, C 5)$ & \\
\hline & $59,5-48,5(d, C 6)$ & \\
\hline & $47,0-42,0(\mathrm{~s}, \mathrm{C} 5)$ & \\
\hline \multirow[t]{4}{*}{$22[7,8 \mathrm{EP}]$} & $52,0-47,0(d, c 6)$ & 100,0 \\
\hline & $55,0-52,0(d, c 7)$ & \\
\hline & $57,0-55,0(d, C 8)$ & \\
\hline & $58,0-56,0(d, C 1)$ & \\
\hline \multirow[t]{3}{*}{$23[1,10 \mathrm{EP}]$} & $63,0-60,0(s, C 10)$ & 100,0 \\
\hline & $44,0-40,0(s, C 5)$ & \\
\hline & $37,2-23,3(\mathrm{~s}, \mathrm{C} 3)$ & \\
\hline \multirow[t]{3}{*}{ 42[sem substituinte] } & $40,5-31,2(s, C 6)$ & 100,0 \\
\hline & $37,9-34,5(\mathrm{~s}, \mathrm{C} 11)$ & \\
\hline & $57,0-45,0(\mathrm{~s}, \mathrm{C} 2)$ & \\
\hline \multirow[t]{3}{*}{$43[4,6,8 E N]$} & $147,0-144,0(s, C 5)$ & 100,0 \\
\hline & $139,0-136,0(s, \mathrm{c} 7)$ & \\
\hline & $155,0-151,0(\mathrm{~s}, \mathrm{C} 9)$ & \\
\hline
\end{tabular}


Tabela 15. Continuação

\begin{tabular}{|c|c|c|}
\hline Subesqueleto & $\begin{array}{c}\text { Faixas de deslocamentos químicos } \\
(\mathrm{ppm})\end{array}$ & $\begin{array}{c}\text { Percentual de } \\
\text { Reconhecimento (\%) } \\
\end{array}$ \\
\hline & $55,5-33,9(\mathrm{~d}, \mathrm{C} 2)$ & \\
\hline \multirow[t]{4}{*}{$44[4,6,8 \mathrm{EN}]$} & $146,8-134,8(\mathrm{~s}, \mathrm{C} 5)$ & 100,0 \\
\hline & $139,3-132,6(s, C 7)$ & \\
\hline & $157,8-141,5(\mathrm{~s}, \mathrm{C} 9)$ & \\
\hline & $30,0-26,0 \quad(d, c 1)$ & \\
\hline \multirow[t]{4}{*}{39 [sem substituintes] } & $18,0-9,0(t, C 2)$ & 100,0 \\
\hline & $28,0-22,0(d, C 3)$ & \\
\hline & $46,0-40,0(s, C 10)$ & \\
\hline & $29,1-27,2$ (d, C1) & \\
\hline \multirow[t]{6}{*}{$39[8,12$ FURANO $]$} & $16,7-10,8(t, C 2)$ & 100,0 \\
\hline & $26,7-23,1$ (d, c3) & \\
\hline & $154,3-152,5(\mathrm{~s}, \mathrm{C} 8)$ & \\
\hline & $120,0-119,0(\mathrm{~s}, \mathrm{C} 11)$ & \\
\hline & $139,0-138,0(d, C 12)$ & \\
\hline & $40,2-38,4(s, C 6)$ & \\
\hline \multirow[t]{3}{*}{55 [sem substituintes] } & $55,7-49,0(t, C 7)$ & 100,0 \\
\hline & $68,0-45,2(\mathrm{~s}, \mathrm{C} 8)$ & \\
\hline & $40,2-38,4(s, C 6)$ & \\
\hline \multirow[t]{5}{*}{$55[1(9) \mathrm{EN} ; 100 \times 0]$} & $68,0-45,2(s, C 8)$ & 100,0 \\
\hline & $169,0-157,0$ (s, c9) & \\
\hline & $138,1-131,1(s, C 1)$ & \\
\hline & $192,3-190,1 \quad(d, C 10)$ & \\
\hline & $40,2-38,4(s, C 6)$ & \\
\hline \multirow[t]{3}{*}{$55[40 R]$} & $68,0-45,2(s, C 8)$ & 100,0 \\
\hline & $72,8-69,3(\mathrm{~d}, \mathrm{C} 4)$ & \\
\hline & $40,0-38,4(\mathrm{~s}, \mathrm{C} 6)$ & \\
\hline \multirow[t]{3}{*}{$55[90 R]$} & $59,0-45,2(s, C 8)$ & 100,0 \\
\hline & $89,5-82,5(\mathrm{~s}, \mathrm{C} 9)$ & \\
\hline & $45,5-45,2(s, C 8)$ & \\
\hline \multirow[t]{3}{*}{$55[10,150 \mathrm{OI} ; 10 \mathrm{OR}]$} & $67,6-67,1 \quad(t, C 15)$ & 100,0 \\
\hline & $98,6-92,1$ (d, C10) & \\
\hline & $38,9-38,4(\mathrm{~s}, \mathrm{C} 6)$ & \\
\hline
\end{tabular}


Tabela 15. Continuação

\begin{tabular}{|c|c|c|}
\hline Subesqueleto & $\begin{array}{c}\text { Faixas de deslocamentos } \\
\text { químicos (ppm) }\end{array}$ & $\begin{array}{c}\text { Percentual de } \\
\text { Reconhecimento (\%) } \\
\end{array}$ \\
\hline \multirow{3}{*}{$55[140 \times 0]$} & $40,0-39,4(s, C 6)$ & \\
\hline & $68,0-59,0(\mathrm{~s}, \mathrm{C} 8)$ & 100,0 \\
\hline & $206,6-198,3$ (d, C14) & \\
\hline \multirow{3}{*}{$55[140 \times 0 ; 14 \mathrm{OMe}]$} & $40,2-38,5(s, c 6)$ & \\
\hline & $55,0-51,0(\mathrm{~s}, \mathrm{C} 8)$ & 100,0 \\
\hline & $179,0-173,8(s, C 15)$ & \\
\hline \multirow{4}{*}{$135[1,3 \mathrm{EN} ; 2,3 \mathrm{OXI}]$} & $130,0-127,0(d, C 1)$ & \\
\hline & $141,0-137,0(s, C 3)$ & 100,0 \\
\hline & $156,0-154,0 \quad(s, \mathrm{C} 4)$ & \\
\hline & $139,0-135,0(\mathrm{~s}, \mathrm{C} 2)$ & \\
\hline \multirow{3}{*}{103 [sem substituintes] } & $65,0-62,0(\mathrm{~s}, \mathrm{C} 4)$ & \\
\hline & $55,0-52,0(\mathrm{~s}, \mathrm{C} 7)$ & 100,0 \\
\hline & $42,0-39,0(s, C 11)$ & \\
\hline \multirow{4}{*}{ 99[sem substituintes] } & $48,0-38,0(\mathrm{~s}, \mathrm{C} 6)$ & \\
\hline & $40,0-36,0(s, C 7)$ & 100,0 \\
\hline & $29,0-25,0(t, C 5)$ & \\
\hline & $39,0-35,0 \quad(s, \mathrm{c} 11)$ & \\
\hline \multirow{4}{*}{$133[1,4 \mathrm{OXI} ; 2 \mathrm{EN} ; 2,5 \mathrm{OXI}]$} & $46,0-44,0 \quad(s, \mathrm{C} 10)$ & \\
\hline & $96,0-94,0(\mathrm{~s}, \mathrm{C5})$ & 100,0 \\
\hline & $95,0-92,0 \quad(s, C 4)$ & \\
\hline & $76,0-73,0(t, C 1)$ & \\
\hline \multirow{3}{*}{$132[5,60 X I]$} & $91,0-86,0(\mathrm{~s}, \mathrm{C5})$ & \\
\hline & $83,0-80,0(\mathrm{~s}, \mathrm{C6})$ & 100,0 \\
\hline & $52,0-46,0 \quad(\mathrm{~s}, \mathrm{C} 10)$ & \\
\hline \multirow{3}{*}{$67[6 \mathrm{EN}]$} & $51,0-48,0(\mathrm{~s}, \mathrm{C5})$ & \\
\hline & $128,0-124,0(d, C 6)$ & 100,0 \\
\hline & $145,0-142,0(\mathrm{~s}, \mathrm{C} 7)$ & \\
\hline \multirow{3}{*}{$130[4 \mathrm{EN}]$} & $127,0-124,0 \quad(s, C 4)$ & \\
\hline & $132,0-129,0(\mathrm{~s}, \mathrm{C} 5)$ & 100,0 \\
\hline & $43,0-41,0(\mathrm{~s}, \mathrm{c} 9)$ & \\
\hline \multirow{3}{*}{$54[30 \times 0]$} & $51,0-44,0(\mathrm{~s}, \mathrm{C} 8)$ & \\
\hline & $69,0-65,0(\mathrm{~s}, \mathrm{c} 9)$ & 100,0 \\
\hline & $213,0-207,0(\mathrm{~s}, \mathrm{C} 3)$ & \\
\hline
\end{tabular}


Tabela 15. Continuação

\begin{tabular}{|c|c|c|}
\hline Subesqueleto & $\begin{array}{l}\text { Faixas de deslocamentos } \\
\text { químicos (ppm) }\end{array}$ & $\begin{array}{c}\text { Percentual de } \\
\text { Reconhecimento (\%) }\end{array}$ \\
\hline \multirow{4}{*}{$\begin{array}{c}\text { 2-2'-Bis-Bisabolano } \\
141[1,4,16,190 \times O ; 2,5,17,20 E N]\end{array}$} & $185,0-180,0(s, C 1)$ & \multirow{4}{*}{100,0} \\
\hline & $142,0-139,0 \quad(s, C 2)$ & \\
\hline & $186,0-183,0(s, C 4)$ & \\
\hline & $126,0-122,0(\mathrm{~s}, \mathrm{C} 6)$ & \\
\hline \multirow{6}{*}{$144[10,17 \mathrm{EN} ; 160 \times 0 ; 17 \mathrm{OH}]$} & $127,0-122,0(d, C 10)$ & \multirow{6}{*}{100,0} \\
\hline & $135,0-130,0(s, \mathrm{c} 11)$ & \\
\hline & $165,0-159,0(\mathrm{~s}, \mathrm{C} 16)$ & \\
\hline & $105,0-100,0(s, \mathrm{C} 17)$ & \\
\hline & $154,0-150,0(s, C 24)$ & \\
\hline & $164,0-158,0(s, C 18)$ & \\
\hline \multirow{3}{*}{$\begin{array}{c}\text { 6-8-Guaiano } \\
89[\text { sem substituintes] }\end{array}$} & $15,0-12,0(d, c 6)$ & \multirow{3}{*}{100,0} \\
\hline & $25,0-21,0(d, c 7)$ & \\
\hline & $13,0-10,0 \quad\left(d_{8}, C 8\right)$ & \\
\hline \multirow{4}{*}{$\begin{array}{c}\text { Trifarano } \\
\text { 40[sem substituintes] }\end{array}$} & $51,2-40,7(\mathrm{~s}, \mathrm{C} 3)$ & \multirow{4}{*}{100,0} \\
\hline & $41,0-39,0(s, C 8)$ & \\
\hline & $49,0-46,0(d, C 4)$ & \\
\hline & $42,0-40,0(d, C 7)$ & \\
\hline \multirow{6}{*}{$40[9 E N]$} & $51,2-40,7(\mathrm{~s}, \mathrm{C} 3)$ & \multirow{6}{*}{100,0} \\
\hline & $41,0-39,0 \quad(s, C 8)$ & \\
\hline & $49,0-46,0(d, C 4)$ & \\
\hline & $42,0-40,0(d, C 7)$ & \\
\hline & $152,6-150,3(\mathrm{~s}, \mathrm{C} 9)$ & \\
\hline & $108,5-106,5(t, C 10)$ & \\
\hline \multirow{3}{*}{$\begin{array}{l}\text { Ipionano } \\
69[40 \times 0]\end{array}$} & $48,0-45,0\left(s, C_{10}\right)$ & \multirow{3}{*}{100,0} \\
\hline & $215,0-213,0(s, C 14)$ & \\
\hline & $58,0-54,0(\mathrm{~d}, \mathrm{C} 3)$ & \\
\hline \multirow{8}{*}{$\begin{array}{l}\text { 6-8',15-9'-Bis-Lindenano } \\
145[\text { sem substituintes] }\end{array}$} & $52,0-49,0(\mathrm{~s}, \mathrm{C} 10)$ & \multirow{8}{*}{100,0} \\
\hline & $27,0-24,0(d, C 1)$ & \\
\hline & $17,0-15,0(t, C 2)$ & \\
\hline & $26,0-24,0(d, C 3)$ & \\
\hline & $43,0-41,0(\mathrm{~s}, \mathrm{C} 25)$ & \\
\hline & $25,0-23,0(d, C 16)$ & \\
\hline & $18,0-16,0(\mathrm{t}, \mathrm{C} 17)$ & \\
\hline & $25,0-23,0(d, C 18)$ & \\
\hline
\end{tabular}


Tabela 15. Continuação

\begin{tabular}{|c|c|c|}
\hline Subesqueleto & $\begin{array}{c}\text { Faixas de deslocamentos químicos } \\
(\mathrm{ppm})\end{array}$ & $\begin{array}{c}\text { Percentual de } \\
\text { Reconhecimento (\%) } \\
\end{array}$ \\
\hline & $147,5-141,0(s, C 1)$ & \\
\hline \multirow[t]{4}{*}{$25[1(14) \mathrm{EN}]$} & $53,9-38,2(\mathrm{~s}, \mathrm{C} 5)$ & 100,0 \\
\hline & $50,4-44,0(s, \mathrm{C} 6)$ & \\
\hline & $117,9-113,8(t, C 14)$ & \\
\hline & $201,5-190,1 \quad(s, C 3)$ & \\
\hline \multirow[t]{5}{*}{$25[1 \mathrm{EN} ; 30 \times \mathrm{O}]$} & $127,8-126,5(d, C 2)$ & 100,0 \\
\hline & $174,8-166,5(\mathrm{~s}, \mathrm{c} 1)$ & \\
\hline & $44,7-40,4(s, C 5)$ & \\
\hline & $49,5-43,0(\mathrm{~s}, \mathrm{C} 6)$ & \\
\hline & $36,0-34,0(s, C 1)$ & \\
\hline \multirow[t]{3}{*}{$138[5,6 \mathrm{EP}]$} & $67,5-63,0(\mathrm{~s}, \mathrm{C5})$ & 100,0 \\
\hline & $71,5-68,0(\mathrm{~s}, \mathrm{C} 6)$ & \\
\hline & $41,4-40,7(s, \mathrm{C} 1)$ & \\
\hline \multirow[t]{4}{*}{$138[5,100 X I]$} & $53,7-51,7(d, C 6)$ & 100,0 \\
\hline & $78,3-77,5(\mathrm{~s}, \mathrm{C5})$ & \\
\hline & $71,0-66,0(\mathrm{~d}, \mathrm{C} 10)$ & \\
\hline & $39,0-35,0(s, \mathrm{c} 1)$ & \\
\hline \multirow[t]{4}{*}{$138[5(14) \mathrm{EN}]$} & $54,2-51,7(d, C 6)$ & 100,0 \\
\hline & $151,8-145,3(s, C 5)$ & \\
\hline & $110,0-108,9(t, C 14)$ & \\
\hline & $49,0-34,5(\mathrm{~s}, \mathrm{C} 1)$ & \\
\hline \multirow[t]{4}{*}{ 138[9EN; 11OXO; 11OR] } & $160,1-149,1 \quad(s, c 9)$ & 100,0 \\
\hline & $130,3-115,1 \quad(d, C 10)$ & \\
\hline & $167,3-166,1 \quad(s, \mathrm{c} 11)$ & \\
\hline & $137,1-123,0 \quad(d, C 7)$ & \\
\hline \multirow[t]{5}{*}{$138[7,9 \mathrm{EN}]$} & $137,3-117,4(d, C 8)$ & 100,0 \\
\hline & $153,0-135,0 \quad(s, \mathrm{C} 9)$ & \\
\hline & $130,5-117,5(d, C 10)$ & \\
\hline & $49,0-34,5(s, C 1)$ & \\
\hline & $41,4-35,0 \quad(s, C 1)$ & \\
\hline \multirow[t]{3}{*}{$138[8 E N]$} & $54,0-51,7(d, C 6)$ & 100,0 \\
\hline & $156,6-120,6(d, C 8)$ & \\
\hline & $142,6-133,1 \quad(s, C 9)$ & \\
\hline
\end{tabular}


Tabela 15. Continuação

\begin{tabular}{|c|c|c|}
\hline Subesqueleto & $\begin{array}{l}\text { Faixas de deslocamentos químicos } \\
(\mathrm{ppm})\end{array}$ & $\begin{array}{c}\text { Percentual de } \\
\text { Reconhecimento (\%) } \\
\end{array}$ \\
\hline & $33,5-26,3(d, c 7)$ & \\
\hline \multirow[t]{3}{*}{$129[9,15 \mathrm{OXO} ; 15 \mathrm{OR}]$} & $181,3-167,0(s, C 15)$ & 100,0 \\
\hline & $213,1-200,0(\mathrm{~s}, \mathrm{C} 9)$ & \\
\hline & $55,4-48,2(t, C 8)$ & \\
\hline \multirow[t]{4}{*}{$129[90 \times 0 ; 10 E N]$} & $201,0-198,6 \quad(s, C 9)$ & 100,0 \\
\hline & $125,0-123,0(d, C 10)$ & \\
\hline & $155,8-154,0(\mathrm{~s}, \mathrm{C} 11)$ & \\
\hline & $79,5-73,0 \quad(s, \mathrm{c} 7)$ & \\
\hline \multirow[t]{4}{*}{$129[7,110 \mathrm{XI}]$} & $79,3-74,0(s, \mathrm{C} 11)$ & 100,0 \\
\hline & $31,2-24,0(q, C 12)$ & \\
\hline & $31,0-23,3(q, C 13)$ & \\
\hline & $157,1-136,6 \quad(s, \mathrm{c} 1)$ & \\
\hline \multirow[t]{5}{*}{$129[1,3,5,10 \mathrm{EN} ; 1 \mathrm{OR}]$} & $137,6-121,3 \quad(s, \mathrm{c} 3)$ & 100,0 \\
\hline & $139,5-125,9(s, C 6)$ & \\
\hline & $125,3-122,0(d, C 10)$ & \\
\hline & $132,6-130,3(s, c 11)$ & \\
\hline & $51,2-30,2(d, C 6)$ & \\
\hline \multirow[t]{6}{*}{$129[2,10 \mathrm{EN}]$} & $36,0-25,6(d, c 7)$ & 90,9 \\
\hline & $141,8-121,3(d, C 2)$ & \\
\hline & $161,5-129,8(\mathrm{~s}, \mathrm{C} 3)$ & \\
\hline & $154,8-120,4(d, C 10)$ & \\
\hline & $155,8-129,6(s, C 11)$ & \\
\hline & $37,0-30,6(d, c 7)$ & \\
\hline \multirow[t]{6}{*}{$129[10 \mathrm{OH} ; 2,10 \mathrm{EN}]$} & $72,5-68,0(d, C 1)$ & 100,0 \\
\hline & $154,8-124,3(d, C 10)$ & \\
\hline & $139,3-129,6(s, C 11)$ & \\
\hline & $141,8-119,0(d, C 2)$ & \\
\hline & $140,5-131,6(s, C 3)$ & \\
\hline & $41,2-39,7(d, c 6)$ & \\
\hline \multirow[t]{4}{*}{$129[2,7(14) \mathrm{EN}]$} & $144,8-120,6(d, C 2)$ & 100,0 \\
\hline & $150,8-133,6(s, C 3)$ & \\
\hline & $154,3-135,3(\mathrm{~s}, \mathrm{C} 7)$ & \\
\hline & $109,5-107,0(t, C 14)$ & \\
\hline
\end{tabular}


Tabela 15. Continuação

\begin{tabular}{|c|c|c|}
\hline Subesqueleto & $\begin{array}{c}\text { Faixas de deslocamentos químicos } \\
(\mathrm{ppm})\end{array}$ & $\begin{array}{c}\text { Percentual de } \\
\text { Reconhecimento (\%) }\end{array}$ \\
\hline & $201,1-186,6(s, C 1)$ & \\
\hline \multirow[t]{5}{*}{$129[10 \times O ; 2,10 \mathrm{EN}]$} & $133,6-127,0(d, c 2)$ & 90,0 \\
\hline & $161,5-144,6(s, C 3)$ & \\
\hline & $153,1-123,5(d, C 10)$ & \\
\hline & $155,8-130,0(\mathrm{~s}, \mathrm{C} 11)$ & \\
\hline & $54,7-51,9(d, c 7)$ & \\
\hline \multirow[t]{5}{*}{$105[1(10), 4 \mathrm{EN}]$} & $133,6-127,0(d, C 5)$ & 100,0 \\
\hline & $134,1-129,5(\mathrm{~s}, \mathrm{C} 4)$ & \\
\hline & $135,5-132,8(s, \mathrm{C} 10)$ & \\
\hline & $132,0-127,5(d, C 1)$ & \\
\hline & $72,0-64,3(d, C 5)$ & \\
\hline \multirow[t]{4}{*}{$105[1(10) ; 4,5 E P]$} & $60,5-59,0 \quad(s, C 4)$ & 100,0 \\
\hline & $129,6-121,9(d, C 1)$ & \\
\hline & $140,8-130,4(s, C 10)$ & \\
\hline & $59,9-56,0 \quad(s, \mathrm{C} 4)$ & \\
\hline \multirow[t]{4}{*}{$105[1,10 ; 4,5 \mathrm{EP}]$} & $66,9-61,7(d, C 5)$ & 100,0 \\
\hline & $66,3-60,9(d, c 1)$ & \\
\hline & $62,9-61,4(s, C 10)$ & \\
\hline & $146,8-139,1 \quad(s, C 10)$ & \\
\hline \multirow[t]{4}{*}{$105[4(15) ; 10(14) E N]$} & $117,9-110,5(t, C 14)$ & 100,0 \\
\hline & $153,5-145,5(\mathrm{~s}, \mathrm{C} 4)$ & \\
\hline & $119,4-112,8(t, C 15)$ & \\
\hline & $207,1-204,3(s, C 8)$ & \\
\hline \multirow[t]{5}{*}{$105[1(10), 7(11) \mathrm{EN} ; 80 \times 0]$} & $156,5-126,6(\mathrm{~s}, \mathrm{C})$ & 100,0 \\
\hline & $139,8-130,3(s, C 11)$ & \\
\hline & $138,1-130,0(s, \mathrm{C} 10)$ & \\
\hline & $133,8-129,6(d, C 1)$ & \\
\hline & $130,0-122,4(d, C 1)$ & \\
\hline \multirow[t]{3}{*}{$105[1(10), 4(15) E N]$} & $163,3-133,5(s, C 10)$ & 100,0 \\
\hline & $153,3-142,3(\mathrm{~s}, \mathrm{C} 4)$ & \\
\hline & $122,5-109,0(t, C 15)$ & \\
\hline
\end{tabular}


Tabela 15. Continuação

\begin{tabular}{|c|c|c|}
\hline Subesqueleto & $\begin{array}{c}\text { Faixas de deslocamentos químicos } \\
(\mathrm{ppm})\end{array}$ & $\begin{array}{c}\text { Percentual de } \\
\text { Reconhecimento (\%) }\end{array}$ \\
\hline & $161,3-148,6(s, C 8)$ & \\
\hline \multirow[t]{5}{*}{ 105[1(10),4EN; 8,12FURANO] } & $131,1-128,8(d, C 1)$ & 100,0 \\
\hline & $136,0-127,8(s, C 10)$ & \\
\hline & $140,6-121,9(\mathrm{~s}, \mathrm{C} 4)$ & \\
\hline & $129,1-123,8(d, C 5)$ & \\
\hline & $33,0-30,0(s, c 7)$ & \\
\hline \multirow[t]{4}{*}{ 51[1(6)EN; 7OXO] } & $185,0-172,0(s, C 1)$ & 100,0 \\
\hline & $137,0-135,0 \quad(s, \mathrm{c} 6)$ & \\
\hline & $206,1-201,8(s, C 7)$ & \\
\hline & $57,9-49,4(s, C 4)$ & \\
\hline \multirow[t]{4}{*}{118 [sem substituintes] } & $40,5-39,4(s, C 6)$ & 100,0 \\
\hline & $77,0-68,5(\mathrm{~s}, \mathrm{C} 8)$ & \\
\hline & $64,0-61,0(d, C 7)$ & \\
\hline & $33,7-33,5(s, \mathrm{C} 11)$ & \\
\hline \multirow[t]{3}{*}{$131[8(14) E N]$} & $151,1-150,6(\mathrm{~s}, \mathrm{C} 8)$ & 100,0 \\
\hline & $107,4-107,0(t, C 14)$ & \\
\hline & $48,9-47,7(d, C 1)$ & \\
\hline \multirow[t]{3}{*}{131 [sem substituintes] } & $41,7-41,5(d, C 9)$ & 100,0 \\
\hline & $33,7-33,5(\mathrm{~s}, \mathrm{C} 11)$ & \\
\hline & $33,7-33,5(s, \mathrm{C} 11)$ & \\
\hline \multirow[t]{3}{*}{$131[5 \mathrm{O} \times \mathrm{O} ; 5 \mathrm{OR}]$} & $41,7-41,5(d, C 9)$ & 100,0 \\
\hline & $178,8-173,6(s, C 5)$ & \\
\hline & $37,0-33,0(s, \mathrm{C} 4)$ & \\
\hline \multirow[t]{6}{*}{$137[10(13) \mathrm{EN} ; 11,12 \mathrm{FURANO}]$} & $150,0-145,0 \quad(s, \mathrm{C} 10)$ & 100,0 \\
\hline & $111,0-107,0(t, C 13)$ & \\
\hline & $131,0-121,0 \quad(s, C 8)$ & \\
\hline & $145,0-141,0(d, C 12)$ & \\
\hline & $147,0-138,0(d, C 11)$ & \\
\hline & $36,0-30,0(s, \mathrm{C} 11)$ & \\
\hline \multirow[t]{3}{*}{$31[1,3$ FURANO] } & $148,0-146,0(s, C 3)$ & 100,0 \\
\hline & $126,0-124,0(s, C 15)$ & \\
\hline & $142,0-140,0(d, c 1)$ & \\
\hline
\end{tabular}


Tabela 15. Continuação

\begin{tabular}{|c|c|c|}
\hline Subesqueleto & $\begin{array}{c}\text { Faixas de deslocamentos químicos } \\
(\mathrm{ppm})\end{array}$ & $\begin{array}{c}\text { Percentual de } \\
\text { Reconhecimento (\%) }\end{array}$ \\
\hline & $55,0-52,0(\mathrm{~s}, \mathrm{C} 4)$ & \\
\hline \multirow[t]{4}{*}{$60[70 \times 0 ; 100 H]$} & $31,0-28,0 \quad(d, C 5)$ & 100,0 \\
\hline & $83,0-80,0(s, C 10)$ & \\
\hline & $225,0-207,0(s, C 7)$ & \\
\hline & $39,0-36,0(s, \mathrm{C} 11)$ & \\
\hline \multirow[t]{5}{*}{$83[2,7(13) \mathrm{EN} ; 12 \mathrm{OXO}]$} & $171,0-167,0(\mathrm{~s}, \mathrm{c} 2)$ & 100,0 \\
\hline & $133,0-130,1 \quad(s, C 3)$ & \\
\hline & $145,0-141,0(s, C 7)$ & \\
\hline & $113,0-111,0(t, C 13)$ & \\
\hline & $86,0-84,0 \quad(s, \mathrm{C} 7)$ & \\
\hline \multirow[t]{6}{*}{$140[3,19 ; 7,210 \times 1 ; 160 \times 0]$} & $80,0-75,0(s, \mathrm{c} 3)$ & 100,0 \\
\hline & $154,0-151,0(\mathrm{~s}, \mathrm{C} 19)$ & \\
\hline & $162,0-158,0(s, C 21)$ & \\
\hline & $156,0-154,0(s, \mathrm{C} 23)$ & \\
\hline & $163,0-161,0(s, C 16)$ & \\
\hline & $62,5-44,2(\mathrm{~s}, \mathrm{C} 8)$ & \\
\hline \multirow[t]{3}{*}{$52[$ sem substituintes] } & $59,2-47,7(s, \mathrm{c} 9)$ & 100,0 \\
\hline & $45,0-28,6(d, c 1)$ & \\
\hline & $61,7-45,7$ (s, c9) & \\
\hline \multirow[t]{4}{*}{$52[4(15) E N]$} & $59,2-50,2(\mathrm{~s}, \mathrm{C} 8)$ & 100,0 \\
\hline & $146,3-139,1$ (s, C4) & \\
\hline & $108,5-106,4(t, C 15)$ & \\
\hline & $120,5-111,4(s, C 5)$ & \\
\hline \multirow[t]{5}{*}{ 52[6,11FURANO] } & $150,8-147,0(s, C 6)$ & 100,0 \\
\hline & $110,5-106,6(d, C 10)$ & \\
\hline & $143,1-141,1$ (d, C11) & \\
\hline & $61,7-59,9 \quad(s, C 8)$ & \\
\hline & $58,5-52,5(\mathrm{~s}, \mathrm{C} 9)$ & 100,0 \\
\hline \multirow[t]{5}{*}{ 53[4OXO; 6,11FURANO] } & $197,3-193,8(s, C 4)$ & \\
\hline & $117,4-116,8(\mathrm{~s}, \mathrm{C} 5)$ & \\
\hline & $163,1-162,3(\mathrm{~s}, \mathrm{C} 6)$ & \\
\hline & $107,5-107,0(d, C 10)$ & \\
\hline & $144,3-144,0(d, C 11)$ & \\
\hline
\end{tabular}


Tabela 15. Continuação

\begin{tabular}{|c|c|c|}
\hline Subesqueleto & $\begin{array}{c}\text { Faixas de deslocamentos químicos } \\
(\mathrm{ppm})\end{array}$ & $\begin{array}{c}\text { Percentual de } \\
\text { Reconhecimento (\%) } \\
\end{array}$ \\
\hline & $92,5-90,3(d, C 2)$ & \\
\hline \multirow[t]{4}{*}{$97[2 \mathrm{OR} ; 2,30 \mathrm{OI} ; 3 \mathrm{EN}]$} & $29,6-18,7(s, \mathrm{C} 11)$ & 100,0 \\
\hline & $153,3-140,1 \quad(d, C 3)$ & \\
\hline & $116,5-113,3(\mathrm{~s}, \mathrm{C} 4)$ & \\
\hline & $20,1-19,5$ (s, C11) & \\
\hline \multirow[t]{3}{*}{$97[10(14) E N]$} & $148,6-146,8(s, C 10)$ & 100,0 \\
\hline & $116,9-115,0 \quad(t, C 14)$ & \\
\hline & $61,5-59,2(s, C 10)$ & \\
\hline \multirow[t]{3}{*}{$97[10,14 \mathrm{EP}]$} & $57,4-51,5(t, C 14)$ & 100,0 \\
\hline & $21,7-18,5(s, \mathrm{C} 11)$ & \\
\hline & $29,6-18,7(s, C 11)$ & \\
\hline \multirow[t]{3}{*}{$97[2,15 \mathrm{OR} ; 2,3 \mathrm{OXI}]$} & $63,5-63,0 \quad(t, C 15)$ & 100,0 \\
\hline & $92,5-90,3(d, C 2)$ & \\
\hline & $29,6-18,7$ (s, C11) & \\
\hline \multirow[t]{3}{*}{$97[2,3 \mathrm{OXI} ; 3 \mathrm{EN}]$} & $153,3-133,8(d, C 3)$ & 94,0 \\
\hline & $124,5-111,9(\mathrm{~s}, \mathrm{C} 4)$ & \\
\hline & $51,0-48,0(\mathrm{~s}, \mathrm{C} 4)$ & \\
\hline \multirow[t]{2}{*}{124 [sem substituintes] } & $40,0-33,0(s, C 9)$ & 100,0 \\
\hline & $41,0-38,0(d, C 9)$ & \\
\hline \multirow[t]{2}{*}{$139[6,90 \times 1]$} & $90,6-86,3(\mathrm{~s}, \mathrm{C} 6)$ & \\
\hline & $83,8-75,4(\mathrm{~s}, \mathrm{C} 9)$ & 100,0 \\
\hline \multirow[t]{2}{*}{$139[10,11 \mathrm{IN}]$} & $90,0-87,0(s, \mathrm{c} 10)$ & \\
\hline & $72,0-68,0(d, C 11)$ & 100,0 \\
\hline & $126,1-123,0(d, C 4)$ & \\
\hline \multirow[t]{4}{*}{$139[4,6 \mathrm{EN}]$} & $131,3-130,6(s, C 5)$ & 100,0 \\
\hline & $146,6-142,3(s, C 6)$ & \\
\hline & $118,6-116,0(d, C 7)$ & \\
\hline & $127,5-122,9(d, C 4)$ & \\
\hline \multirow[t]{3}{*}{$139[4 \mathrm{EN} ; 6,90 \times \mathrm{O}]$} & $140,3-132,6(s, C 5)$ & 100,0 \\
\hline & $90,6-86,3(\mathrm{~s}, \mathrm{C} 6)$ & \\
\hline & $83,8-75,4(\mathrm{~s}, \mathrm{C} 9)$ & \\
\hline
\end{tabular}


Tabela 15. Continuação

\begin{tabular}{|c|c|c|}
\hline Subesqueleto & $\begin{array}{l}\text { Faixas de deslocamentos } \\
\text { químicos (ppm) }\end{array}$ & $\begin{array}{c}\text { Percentual de } \\
\text { Reconhecimento (\%) }\end{array}$ \\
\hline & $143,8-143,6\left(s, c_{2}\right)$ & \\
\hline \multirow[t]{4}{*}{$95[1,3,5 \mathrm{EN} ; 6 \mathrm{OR}]$} & $136,0-128,5(\mathrm{~s}, \mathrm{C} 3)$ & 100,0 \\
\hline & $126,4-120,4(s, C 5)$ & \\
\hline & $155,5-148,5(\mathrm{~s}, \mathrm{C} 6)$ & \\
\hline & $128,0-123,0(d, C 4)$ & \\
\hline \multirow[t]{5}{*}{$136[4,6 \mathrm{EN} ; 90 \times 0]$} & $132,0-129,0(s, C 5)$ & 100,0 \\
\hline & $147,0-141,0(s, \mathrm{C} 6)$ & \\
\hline & $117,0-113,0(d, C 7)$ & \\
\hline & $208,0-205,0(s, C 9)$ & \\
\hline & $193,3-192,3(d, C 12)$ & \\
\hline \multirow[t]{4}{*}{ 14[7EN;12OXO] } & $140,1-137,1 \quad(s, C 8)$ & 100,0 \\
\hline & $155,8-152,6(d, C 7)$ & \\
\hline & $42,5-35,0(\mathrm{~s}, \mathrm{C} 10)$ & \\
\hline & $45,7-36,5(d, C 5)$ & \\
\hline \multirow[t]{3}{*}{$123[6,7 \mathrm{EP}]$} & $62,4-60,7(d, C 6)$ & \\
\hline & $63,2-60,5(s, c 7)$ & 100,0 \\
\hline & $37,7-36,5(\mathrm{~s}, \mathrm{C} 11)$ & \\
\hline & $38,0-35,0\left(s, C_{11}\right)$ & \\
\hline \multirow[t]{5}{*}{$123[2,6 \mathrm{EN}]$} & $143,0-122,1 \quad(d, C 2)$ & 100,0 \\
\hline & $143,0-132,8(d, C 3)$ & \\
\hline & $151,6-123,9(d, C 6)$ & \\
\hline & $139,0-133,3(\mathrm{~s}, \mathrm{C} 7)$ & \\
\hline & $151,6-126,0(d, C 6)$ & \\
\hline \multirow[t]{5}{*}{$123[6,9 \mathrm{EN}]$} & $139,3-133,3(\mathrm{~s}, \mathrm{C} 7)$ & 100,0 \\
\hline & $128,1-127,0(d, C 9)$ & \\
\hline & $160,3-140,8(d, C 10)$ & \\
\hline & $38,0-35,0(\mathrm{~s}, \mathrm{C} 11)$ & \\
\hline & $45,0-44,0 \quad(s, C 7)$ & \\
\hline \multirow[t]{4}{*}{$71[1,3,5 \mathrm{EN} ; 1,100 \mathrm{XI}]$} & $88,0-85,0(\mathrm{~s}, \mathrm{C} 10)$ & 100,0 \\
\hline & $153,0-150,0(s, \mathrm{C} 1)$ & \\
\hline & $138,0-136,0(\mathrm{~s}, \mathrm{C} 3)$ & \\
\hline & $131,0-129,0(\mathrm{~s}, \mathrm{C} 6)$ & \\
\hline
\end{tabular}


Tabela 15. Continuação

\begin{tabular}{|c|c|c|}
\hline Subesqueleto & $\begin{array}{c}\text { Faixas de deslocamentos } \\
\text { químicos (ppm) } \\
\end{array}$ & $\begin{array}{c}\text { Percentual de } \\
\text { Reconhecimento (\%) } \\
\end{array}$ \\
\hline & $52,0-44,0 \quad(s, C 7)$ & \\
\hline \multirow[t]{3}{*}{$71[1,3,5 \mathrm{EN}]$} & $135,3-128,6(s, \mathrm{C} 6)$ & 100,0 \\
\hline & $153,3-150,1 \quad(s, C 3)$ & \\
\hline & $48,7-45,2(s, C 1)$ & \\
\hline \multirow[t]{5}{*}{$113[8(12) \mathrm{EN}]$} & $55,5-54,2(\mathrm{~s}, \mathrm{C} 2)$ & 100,0 \\
\hline & $55,7-54,5(s, C 6)$ & \\
\hline & $154,3-145,3(\mathrm{~s}, \mathrm{C} 8)$ & \\
\hline & $123,0-107,0$ (t, C12) & \\
\hline & $52,2-39,7(s, c 6)$ & \\
\hline \multirow[t]{6}{*}{$72[5,100 X I ; 11,12 E P]$} & $70,1-57,2(d, C 5)$ & 100,0 \\
\hline & $79,6-73,4 \quad(d, C 10)$ & \\
\hline & $49,5-42,2(\mathrm{~s}, \mathrm{C} 7)$ & \\
\hline & $65,9-63,5(\mathrm{~s}, \mathrm{C} 11)$ & \\
\hline & $49,0-41,4(t, C 12)$ & \\
\hline & $82,0-80,0(d, C 1)$ & \\
\hline \multirow[t]{4}{*}{$72[1,110 \times I]$} & $93,0-90,0(s, C 11)$ & 100,0 \\
\hline & $53,0-51,0 \quad(s, C 7)$ & \\
\hline & $47,0-44,0 \quad(\mathrm{~s}, \mathrm{C} 6)$ & \\
\hline & $51,9-44,0 \quad(s, \mathrm{C} 7)$ & \\
\hline \multirow[t]{3}{*}{112 [sem substituintes] } & $60,2-51,9(d, C 1)$ & 100,0 \\
\hline & $55,7-47,9(d, C 10)$ & \\
\hline & $51,9-44,9(\mathrm{~s}, \mathrm{C} 7)$ & \\
\hline \multirow[t]{4}{*}{$112[30 \times 0]$} & $56,7-51,9(\mathrm{~d}, \mathrm{C} 1)$ & 100,0 \\
\hline & $55,7-48,2(d, C 10)$ & \\
\hline & $222,8-216,6(\mathrm{~s}, \mathrm{C} 3)$ & \\
\hline & $46,0-43,0(s, C 7)$ & \\
\hline \multirow[t]{3}{*}{$112[50 \times 0]$} & $61,0-58,0(\mathrm{~d}, \mathrm{C} 1)$ & 100,0 \\
\hline & $49,0-46,0(d, C 10)$ & \\
\hline & $222,0-217,0 \quad(s, C 5)$ & \\
\hline
\end{tabular}


Tabela 15. Continuação

\begin{tabular}{|c|c|c|}
\hline Subesqueleto & $\begin{array}{c}\text { Faixas de deslocamentos } \\
\text { químicos (ppm) }\end{array}$ & $\begin{array}{c}\text { Percentual de } \\
\text { Reconhecimento (\%) }\end{array}$ \\
\hline & $46,2-33,2(s, \mathrm{c} 11)$ & \\
\hline \multirow[t]{5}{*}{$83[5,13$ FURANO $]$} & $130,8-119,6(\mathrm{~s}, \mathrm{C} 7)$ & 100,0 \\
\hline & $128,6-117,5(s, \mathrm{C} 6)$ & \\
\hline & $148,6-136,1 \quad(d, c 13)$ & \\
\hline & $149,0-137,3(d, C 5)$ & \\
\hline & $38,0-37,7(5, \mathrm{C} 3)$ & \\
\hline \multirow[t]{3}{*}{$83[3(12) \mathrm{EN}]$} & $147,8-137,6(s, C 12)$ & 80,0 \\
\hline & $113,0-112,8(t, C 11)$ & \\
\hline & $45,2-43,2(d, C 1)$ & \\
\hline \multirow[t]{4}{*}{ 63[sem substituinte] } & $40,2-38,0(d, C 9)$ & 100,0 \\
\hline & $44,0-43,2(s, C 4)$ & \\
\hline & $49,2-45,2(d, C 5)$ & \\
\hline & $46,0-43,0(s, C 4)$ & \\
\hline \multirow[t]{4}{*}{$63[6,100 \mathrm{XI}]$} & $75,0-71,0(\mathrm{~s}, \mathrm{C} 6)$ & 100,0 \\
\hline & $77,0-73,0(\mathrm{~s}, \mathrm{C} 10)$ & \\
\hline & $42,0-39,0(d, c 9)$ & \\
\hline & $44,0-43,7$ (s, C1) & \\
\hline \multirow[t]{5}{*}{$96[2,8 \mathrm{EN}]$} & $140,6-138,6(d, C 2)$ & 100,0 \\
\hline & $127,4-127,0 \quad(s, \mathrm{C} 3)$ & \\
\hline & $149,5-147,8 \quad(s, C 8)$ & \\
\hline & $125,9-121,5(d, c 9)$ & \\
\hline & $48,0-44,0 \quad(d, C 2)$ & \\
\hline \multirow[t]{4}{*}{ 47[sem substituinte] } & $57,0-53,0 \quad(s, C 1)$ & 100,0 \\
\hline & $55,0-46,0 \quad(t, C 12)$ & \\
\hline & $42,0-38,0\left(s, C_{13}\right)$ & \\
\hline & $49,0-39,2(s, \mathrm{c} 6)$ & \\
\hline \multirow[t]{4}{*}{104 [sem substituinte] } & $50,9-32,0(d, c 7)$ & 100,0 \\
\hline & $32,0-21,8(t, C 8)$ & \\
\hline & $44,7-39,7 \quad(s, \mathrm{C} 3)$ & \\
\hline & $35,5-33,7(d, C 1)$ & \\
\hline
\end{tabular}


Tabela 15. Continuação

\begin{tabular}{|c|c|c|}
\hline Subesqueleto & $\begin{array}{l}\text { Faixas de deslocamentos químicos } \\
(\mathrm{ppm})\end{array}$ & $\begin{array}{c}\text { Percentual de } \\
\text { Reconhecimento (\%) }\end{array}$ \\
\hline \multirow{4}{*}{$142[5(13) E N]$} & $42,0-38,0(s, c 6)$ & \multirow{4}{*}{100,0} \\
\hline & $86,0-77,0$ (s, c9) & \\
\hline & $161,0-158,0(s, C 5)$ & \\
\hline & $106,0-102,0(t, C 13)$ & \\
\hline \multirow{6}{*}{$143[5(13), 16,19 \mathrm{EN} ; 18,210 \times \mathrm{O}]$} & $42,0-38,0 \quad(s, C 6)$ & \multirow{6}{*}{100,0} \\
\hline & $163,0-158,1(s, C 5)$ & \\
\hline & $105,0-102,0(t, C 13)$ & \\
\hline & $124,0-116,0(s, C 16)$ & \\
\hline & $186,0-181,0(\mathrm{~s}, \mathrm{C} 18)$ & \\
\hline & $185,0-177,0(\mathrm{~s}, \mathrm{C} 21)$ & \\
\hline \multirow{4}{*}{$45[1,3,5 \mathrm{EN} ; 80 \times 0]$} & $50,7-45,7(s, c 7)$ & \multirow{4}{*}{100,0} \\
\hline & $213,8-206,6(s, C 8)$ & \\
\hline & $163,6-154,0 \quad(s, C 5)$ & \\
\hline & $150,1-142,6(s, c 9)$ & \\
\hline \multirow{4}{*}{$101[1,140 \times I]$} & $38,0-35,0 \quad(s, C 11)$ & \multirow{4}{*}{100,0} \\
\hline & $97,0-94,0(s, C 1)$ & \\
\hline & $84,0-79,0(d, C 14)$ & \\
\hline & $61,0-57,0(d, c 5)$ & \\
\hline \multirow{4}{*}{$89[1(10) \mathrm{EN}]$} & $42,9-41,5 \quad(s, c 9)$ & \multirow{4}{*}{100,0} \\
\hline & $142,0-138,6(d, C 10)$ & \\
\hline & $145,6-139,5(s, C 1)$ & \\
\hline & $50,4-48,2(d, C 7)$ & \\
\hline \multirow{3}{*}{$46[$ sem substituintes] } & $60,0-42,0 \quad\left(s, c_{10}\right)$ & \multirow{3}{*}{100,0} \\
\hline & $24,0-20,0\left(s, C_{11}\right)$ & \\
\hline & $41,0-34,0(s, C 5)$ & \\
\hline \multirow{4}{*}{$49[$ sem substituintes] } & $53,0-50,0(\mathrm{~s}, \mathrm{C} 6)$ & \multirow{4}{*}{100,0} \\
\hline & $46,0-44,0(s, C 7)$ & \\
\hline & $24,0-19,0(s, C 4)$ & \\
\hline & $33,0-31,0(s, C 11)$ & \\
\hline \multirow{4}{*}{ 50[sem substituintes] } & $73,6-73,1 \quad(s, c 5)$ & \multirow{4}{*}{100,0} \\
\hline & $66,4-65,5(s, \mathrm{C} 1)$ & \\
\hline & $49,7-46,0(s, C 4)$ & \\
\hline & $30,0-29,2(d, c 9)$ & \\
\hline
\end{tabular}


Tabela 15. Continuação

\begin{tabular}{|c|c|c|}
\hline Subesqueleto & $\begin{array}{c}\text { Faixas de deslocamentos } \\
\text { químicos (ppm) } \\
\end{array}$ & $\begin{array}{c}\text { Percentual de } \\
\text { Reconhecimento (\%) } \\
\end{array}$ \\
\hline \multirow[t]{3}{*}{127 [sem substituintes] } & $52,2-50,0(\mathrm{~d}, \mathrm{C} 1)$ & \\
\hline & $42,0-39,0(d, C 5)$ & 100,0 \\
\hline & $44,0-42,0(\mathrm{~s}, \mathrm{C} 6)$ & \\
\hline \multirow{5}{*}{$127[2(15) \mathrm{EN}]$} & $52,0-50,0(d, C 1)$ & \\
\hline & $42,0-39,0(d, C 5)$ & 100,0 \\
\hline & $44,0-42,0(\mathrm{~s}, \mathrm{C} 6)$ & \\
\hline & $152,5-151,0 \quad(s, C 2)$ & \\
\hline & $107,0-106,0(t, C 15)$ & \\
\hline \multirow{5}{*}{100 [sem substituintes] } & $37,5-33,5(\mathrm{~s}, \mathrm{C} 4)$ & \\
\hline & $34,7-33,0(s, C 11)$ & 100,0 \\
\hline & $49,9-39,5(\mathrm{~d}, \mathrm{C} 1)$ & \\
\hline & $49,0-38,2(d, C 9)$ & \\
\hline & $48,7-35,2(\mathrm{t}, \mathrm{C} 14)$ & \\
\hline \multirow[t]{3}{*}{$98[5 E N]$} & $40,0-37,0 \quad(s, C 4)$ & \\
\hline & $36,0-33,0(t, \mathrm{C} 11)$ & 100,0 \\
\hline & $129,0-127,0 \quad(s, C 5)$ & \\
\hline
\end{tabular}




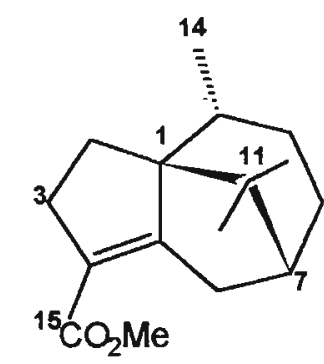

Esqueleto CIPERANO
Phytochemistry, 1997, $\underline{45}, 149$ Joannesia princeps (Euphorbiaceae)

RMN ${ }^{13} \mathrm{C}\left(\mathrm{CDCl}_{3}, \mathbf{C 1} 1 \mathrm{C15}\right): 67,9$ (s); 25,7 (t); 36,7 (t); 123,3 (s); 169,3 (s); 31,1 (t); 48,2 (d); 27,0 (t); 27,9 (t); 35,9 (d); 41,6 (s); 26,2 (q); 19,3 (q); 17,9 (q); 166,0 (s); OMe - 50,9 (q).

SUBESTRUTRURA PROPOSTA PELO SISCONST:

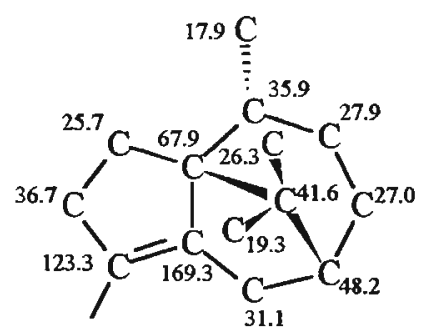

PROBABILDADE DE ESQUELETO (\%)

\begin{tabular}{lccccc}
\hline ESQUELETO & SISCONST & C13MACH & SISOCBOT & REGRAS & GLOBAL \\
\hline \hline CIPERANO & 50.5 & 91.4 & - & 100.0 & 38.1 \\
GIMNOMITRANO & - & - & 100.0 & - & 8.0 \\
GUAIANO & 7.8 & 8.6 & - & - & 4.6 \\
OUTROS & 12.0 & - & - & - & 3.8 \\
EUDESMANO & 11.6 & - & - & - & 3.7 \\
EREMOFLANO & 8.4 & - & - & - & 2.7 \\
CAROTANO & 5.0 & - & - & - & 1.6 \\
SEICHELANO & 1.8 & - & - & - & 0.6 \\
\hline
\end{tabular}

SUBESQUELETO : $78[$ sem substituintes] $-100.0 \%$ 


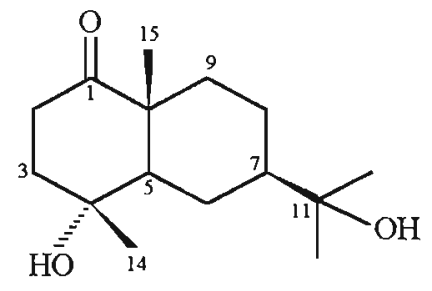

Esqueleto EUDESMANO
Phytochemistry, 1996, $\underline{4}, 815$ Artemisia eriopoda (Compositae)

RMN ${ }^{13}$ C (acetona d-6, C1-C15): 215,6 (s); 41,1 (t); 35,9 (t); 73,7 (s); 43,8 (d); 22,1 (t); 42,5 (d); 21,6 $(\mathrm{t}) ; 33,6(\mathrm{t}) ; 46,6$ (s); 70,7 (s); 23,4 (q); 29,9 (q); 29,7 (q); 29,0 (q).

SUBESTRUTURAS PROPOSTAS PELO SISCONST:<smiles>CCCC(CC(C)C(C)(O)CC)C(C)(C)O</smiles><smiles>CCC(=O)C(C)(C)CCC(C)C(C)(C)O</smiles><smiles>CC1(Cl)CCC(C(C)(C)O)CC1</smiles>

PROBABILIDADE DE ESQUELETO (\%)

\begin{tabular}{lccccc}
\hline ESQUELETO & SISCONST & C13MACH & SISOCBOT & REGRAS & GLOBAL \\
\hline \hline EUDESMANO & 38.7 & 29.7 & 25.0 & 100.0 & 57.5 \\
BISABOLANO & 11.1 & 20.1 & 6.3 & - & 8.9 \\
ESQ31 & 19.3 & - & - & - & 6.2 \\
CADINANO & 3.3 & 10.0 & 9.4 & - & 4.2 \\
GUAIANO & 8.5 & - & - & - & 2.7 \\
LONGICICLANO & - & 10.6 & -- & - & 2.5 \\
ESTERPURANO & - & 10.1 & - & - & 2.4 \\
CEDRANO & - & 9.8 & - & - & 2.3 \\
\hline
\end{tabular}

SUBESQUELETOS : $1[4,11 \mathrm{OR}]-100.0 \% ; 1[11 \mathrm{OR}]-90.6 \%$ 


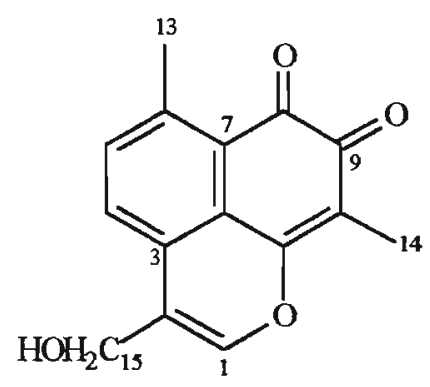

Phytochemistry, 1996, $\underline{43}, 425$

Esqueleto CADINANO

$\mathrm{RMN}^{13} \mathrm{C}\left(\mathrm{CDCl}_{3}, \mathbf{C 1}-\mathrm{C15}\right): 143,1$ (d); 117,5 (s); 129,1 (s); 130,4 (d); 137,6 (d); 148,0 (s); 126,5 (s); $182,5(\mathrm{~s}) ; 178,7$ (s); 113,8 (s); 163,7 (s); 12,1 (s); 23,4 (q); 7,7 (q); 58,3 (t).

SUBESTRUTURAS PROPOSTAS PELO SISCONST:<smiles>C=C(C)C(=O)C(=O)c1c(C)cccc1C</smiles><smiles>C=Cc1cccc(C)c1C(=O)C(C)=O</smiles><smiles>C=C(C)C1=CC(=O)C(=O)C(C)=C1OC</smiles>

PROBABLIDADE DE ESQUELETO (\%)

\begin{tabular}{lccccc}
\hline \multicolumn{1}{c}{ ESQUELETO } & SISCONST & C13MACH & SISOCBOT & REGRAS & GLOBAL \\
\hline \hline CADINANO & 100.0 & 43.1 & - & 50.0 & 60.3 \\
14(->6)-EREMOFLANO & - & - & - & 50.0 & 18.0 \\
ALIACANO & - & 10.1 & - & - & 2.4 \\
GERMACRANO & - & 10.0 & - & - & 2.4 \\
BISABOLANO & - & 9.8 & - & - & 2.3 \\
ESQ62 & - & 9.0 & - & - & 2.2 \\
EREMOFILANO & - & 9.0 & - & - & 2.2 \\
LAURANO & - & 8.9 & - & - & 2.1 \\
\hline
\end{tabular}

SUBESQUELETOS : $10[2 \mathrm{OXO} ; 1(6), 7,9 \mathrm{EN}]-100.0 \% ; 21[8,12$ FURANO $]-100.0 \%$ 


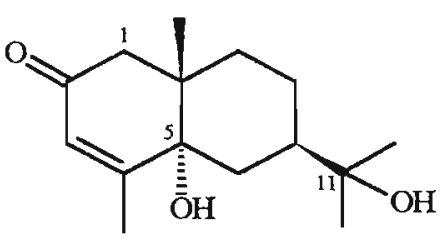

Esqueleto EUDESMANO
Phytochemistry, 1996, $\underline{43}, 81$

Artemisia eriopoda (Compositae)

$\mathrm{RMN}^{13} \mathrm{C}\left(\mathrm{CDCl}_{3}, \mathrm{C1}-\mathrm{C15}\right) ; 4$ 42,3 (t); 199,0 (s); 125,4 (d); 167,0 (s); 76,0 (s); 34,3 (t); 47,0 (d); 22,7 (t); $35,0(\mathrm{t}) ; 40,1(\mathrm{~s}) ; 72,6(\mathrm{~s}) ; 25,5$ (q); 28,6 (q); 22,7 (q); 18,8(q).

SUBESTRUTURAS PROPOSTAS PELO SISCONST:
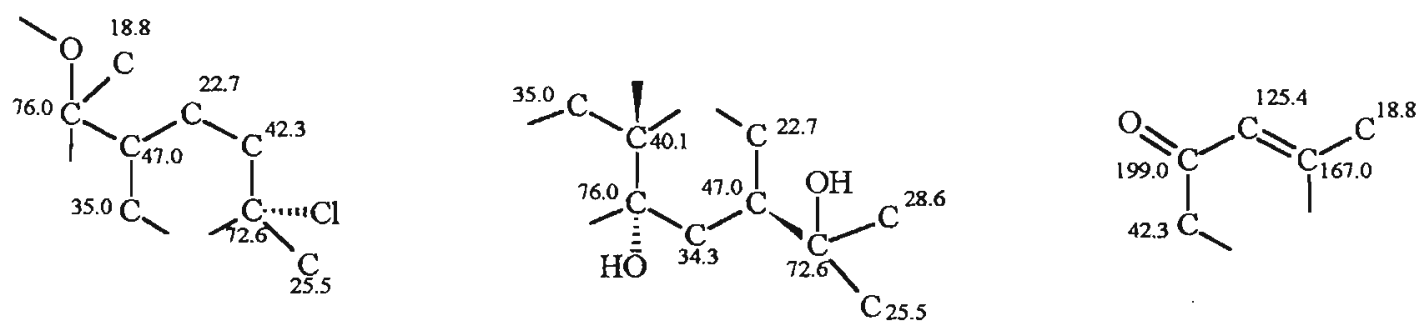

PROBABILIDADE DE ESQUELETO (\%)

\begin{tabular}{lccccc}
\hline ESQUELETO & SISCONST & C13MACH & SISOCBOT & REGRAS & GLOBAL \\
\hline \hline EUDESMANO & 30.9 & 20.2 & 25.0 & 90.6 & 49.4 \\
EREMOFILANO & 27.3 & - & - & - & 8.7 \\
FARNESANO & - & 19.8 & 12.5 & - & 5.8 \\
CADINANO & - & 20.0 & 9.4 & - & 5.6 \\
ESTERPURANO & - & 9.9 & - & - & 2.4 \\
VETISPIRANO & - & 9.8 & - & - & 2.4 \\
ESQ6 & - & 9.7 & - & - & 2.3 \\
ESQ31 & 7.0 & - & - & - & 2.2 \\
\hline
\end{tabular}

SUBESQUELETO: 1 [11OR] - $90.6 \%$ 
<smiles>COc1c(I)cc2c(c1O)C(C)C(OC)CC2C(C)C</smiles>

Esqueleto CADINANO
Phytochemistry, 1996, $\underline{41}, 1351$

Alangium premnifolium (Alangiaceae)

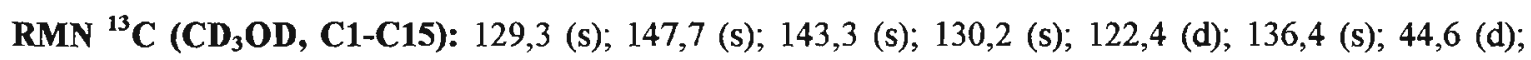
24,1 (t); 76,9 (d); 34,0 (d); 35,9 (d); 19, 3 (q); 22,4 (q); 17,2 (q); 14,6 (q); 107,9 (d); 75,5 (d); 78,3 (d); 71,7 (d); 78,0 (d); 62,8 (t); Gly 3-O: 107,9 (d); 75,5 (d); 78,3 (d); 71,7 (d); 78,0 (d); 62,8 (t); Gly 9-0: 103,5 (d) ; 75,3 (d); 78,4(d); 71,2(d); 78,1(d); 62,5(t).

\section{SUBESTRUTURAS PROPOSTAS PELO SISCONST:}

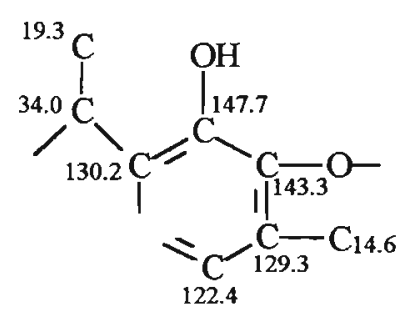<smiles>C=C(OC)C(=C(C)C)C(CC)C(C)C</smiles><smiles>C=C(C=C(C)C)C(C)C(C)C</smiles>

PROBABILIDADE DE ESQUELETO (\%)

\begin{tabular}{lccccc}
\hline ESQUELETO & SISCONST & C13MACH & SISOCBOT & REGRAS & GLOBAL \\
\hline \hline CADINANO & 34.8 & 19.5 & - & 100.0 & 51.8 \\
GUAIANO & 18.7 & 9.5 & - & - & 8.3 \\
ESQ19 & - & 21.7 & - & - & 5.2 \\
EUDESMANO & 8.6 & 9.5 & - & - & 5.0 \\
BISABOLANO & 7.1 & 9.6 & - & - & 4.6 \\
ESQ14 & 3.5 & 10.1 & - & - & 3.5 \\
ESQ12 & - & 10.4 & - & - & 2.5 \\
ISOCOMANO & - & 9.8 & - & - & 2.4 \\
\hline
\end{tabular}

SUBESQUELETO: $10[4,9 \mathrm{EN}]-100.0 \%$

MACRONO: Glicose: 103,5d; 78,4d; 78,3d; 75,3d; 71,7d; 62,8t (erro médio = 0,275); Etóxido: 62,5t; 14,4q (erro médio = 1,450); Glicose: 107,9d; 78,1d; 78,0d; 75,5d; 71,2d; 62,5t (erro médio=1,450). 
<smiles>COc1c(C)cc2c(c1O)C(C)C(C(C)C)CC2OC</smiles>

Phytochemistry, 1996, $\underline{41}, 1351$

Esqueleto CADINANO

Alangium premnifolium (Alangiaceae)

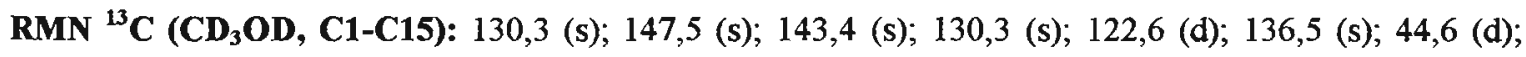
24,1 (t); 77, 2 (d); 34,1 (d); 35,9 (d); 19,3 (q); 22,4 (q); 17,2 (q); 14,8 (q); Xyl: 105,5 (d); 75,5 (d); 77,6 (d); 71,2 (d); 66,9 (t); Gly 3-O: 107,8 (d); 75,4 (d); 78,3 (d); 71,7 (d); 78,0 (d); 62,8 (t); Gly 9-0: 103,8 (d); 75,3 (d); 78,4 (d); 71,7 (d); 78,0 (d); 70,1 (t).

\section{SUBESTRUTURAS PROPOSTAS PELO SISCONST:}<smiles>COc1c(Br)ccc(C(C)C)c1O</smiles><smiles>C=C1C(=C)C(C(C)C)CCC1C</smiles><smiles>CC(C)=CC(=C(C)C)C(C)C(C)C</smiles>

PROBABILIDADE DE ESQUELETO (\%)

\begin{tabular}{lccccc}
\hline ESQUELETO & SISCONST & C13MACH & SISOCBOT & REGRAS & GLOBAL \\
\hline \hline CADINANO & 37.9 & 29.1 & - & 100.0 & 55.1 \\
ESQ19 & - & 22.2 & - & - & 5.3 \\
EUDESMANO & 8.3 & 9.6 & - & - & 5.0 \\
13(->10)-VALERENANO & 14.8 & - & - & - & 4.7 \\
BISABOLANO & 6.5 & 9.5 & - & - & 4.4 \\
ESQ14 & 4.1 & 9.9 & - & - & 3.7 \\
DRIMANO & - & 9.9 & - & - & 2.4 \\
ISOCOMANO & - & 9.7 & - & - & 2.3 \\
\hline
\end{tabular}

SUBESQUELETO: $10[4,9 \mathrm{EN}]-100.0 \%$

MACRONO: Glicose - 103,8d; 77,6d; 77,2d; 75,3d; 71,2d; 62,8t (erro médio = 0,650); Gly (6<-1) Xyl 107,8d; 105,5d; 78,3d; 78,0d; 78,0d; 75,5d; 75,4d; 71,7d; 71,7d; 70,1t; 66,9t (erro médio $=0.686$ ). 
<smiles>COc1cc2c(F)c(OC)cc(C(C)C)c2cc1C</smiles>

Esqueleto CADINANO
Phytochemistry, 1996, $\underline{41}, 1351$ Alangium premnifolium (Alangiaceae)

RMN ${ }^{13}$ C (piridina d-5, C1-C15): 135,0 (s), 108,5 (d), 155,7 (s), 126,5 (s), 125,3 (d), 124,0 (s), 143,5 (s), 113,2 (d), 153,6 (s), 118,3 (s), 28,8 (d), 23,7 (q), 23,8 (q), 17,4 (q), 11,5 (q), Gly 3-O: 104,1 (d), 75,3 (d), 79,2 (d), 71,6 (d), 78,9 (d), 62,7 (t), Gly 9-0: 103,0 (d), 75,1 (d), 78,9 (d), 71,6 (d), 78,9 (d), 62,6 (t).

\section{SUBESTRUTURAS PROPOSTAS PELO SISCONST:}<smiles>COc1cc(C)cc(C(C)(C)C)c1</smiles><smiles>COC(C)C(C)C=C(C)C</smiles>

PROBABILIDADE DE ESQUELETO (\%)

\begin{tabular}{lccccc}
\hline \multicolumn{1}{c}{ ESQUELETO } & SISCONST & CI3MACH & SISOCBOT & REGRAS & GLOBAL \\
\hline \hline CADINANO & 50.9 & 31.6 & - & 100.0 & 59.9 \\
14( $\rightarrow$ 6) EREMOFLLANO & 19.8 & 20.0 & - & - & 11.1 \\
BISABOLANO & 15.6 & - & - & - & 5.0 \\
ALIACANO & 6.1 & 9.6 & - & - & 4.3 \\
SPINIFERIN-1 & - & 9.4 & - & - & 2.8 \\
EUDESMANO & -- & 10.0 & - & - & 2.4 \\
4,5-SECO-EREMOFILANO & - & 9.7 & - & - & 2.3 \\
ESQ45 & -- & 9.7 & -- & -- & 2.3 \\
\hline
\end{tabular}

SUBESQUELETO: $10[1,3,5,7,9 \mathrm{EN}]-100.0 \%$

MACRONO: Gly - 104,1d; 78,9d; 78,9d; 75,3d; 71,6d; 62,7t (erro médio = 0,192); Gly - 103,0d; 79,2d; 78,9d, 75, 1d; 71,6d; 62,6t (erro médio $=0,442$ ). 
<smiles>CC1=C[C@H]2[C@@H](C)CC[C@@](O)(C(C)C)[C@H]2CC1=O</smiles>

Phytochemistry, 1996, 41, 837

Esqueleto CADINANO Artemisia chamaemelifolia (Compositae)

RMN ${ }^{13} \mathrm{C}$ (CDCl 3 , C1-C15): 40,8 (d); 42,4 (t); 199,6 (s); 136,7 (s); 142,9 (d); 45,0 (d); 74,4 (s); 32,5 (t); $29,8(\mathrm{t}) ; 28,1(\mathrm{~d}) ; 33,0(\mathrm{~d}) ; 16,1$ (s); 15,7 (s); 19,3 (s); 16,1 (s).

SUBESTRUTURAS PROPOSTAS PELO SISCONST:<smiles>CC(C)CC(C)(O)C1CC(=O)C(C)(C)CC1C</smiles><smiles>CC(=O)C(C)=CC(C(C)C(C)C)C(C)(C)O</smiles>

PROBABILIDADE DE ESQUELETO (\%)

\begin{tabular}{lccccc}
\hline \multicolumn{1}{c}{ ESQUELETO } & SISCONST & CI3MACH & SISOCBOT & REGRAS & GLOBAL \\
\hline \hline CADINANO & 52.5 & 40.3 & 9.4 & 100.0 & 63.2 \\
AROMADENDRANO & 22.2 & 10.3 & - & - & 9.6 \\
BISABOLANO & 5.1 & 19.8 & 6.3 & - & 6.9 \\
PROTOILUDANO & - & 20.0 & - & - & 4.8 \\
4,5-SECO-GUAIANO & 13.1 & - & - & - & 4.2 \\
ESQ6 & - & 9.6 & - & - & 2.3 \\
EUDESMANO & - & - & 25.0 & - & 2.0 \\
GUAIANO & 2.0 & - & - & - & 0.6 \\
\hline
\end{tabular}

SUBESQUELETOS : $10[30 X 0 ; 4 \mathrm{EN}]-100.0 \% ; 10[11 \mathrm{OR}, 4 \mathrm{EN}]-100.0 \%$ 
<smiles>CC(C)[C@H]1CC[C@@H](C)C23CCC(C)(C=C12)OO3</smiles>

Esqueleto CADINANO
Phytochemistry, 1996, $\underline{42}, 1361$

Bazzania trilobata (Lepidoziaceae)

RMN ${ }^{13} \mathrm{C}\left(\mathrm{CDCl}_{3}, \mathbf{C 1}-\mathbf{C 1 5}\right): 30,6$ (d); 26,7 (t); 19,3 (t); 39,4 (d); 148,5 (s); 126,3 (d); 75,3 (s); 30,0 (t); $21,9(\mathrm{t}) ; 79,3$ (s); 27,5 (d); 16,9 (q); 21,4 (q); 15,4 (q); 22,0 (q).

SUBESTRUTURAS PROPOSTAS PELO SISCONST:

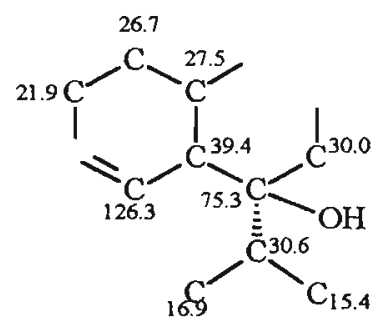<smiles>CC(C)CC(CC(C)C)C(O)(C(C)C)C(C)C</smiles>

PROBABILIDADE DE ESQUELETO (\%)

\begin{tabular}{lccccc}
\hline \multicolumn{1}{c}{ ESQUELETO } & SISCONST & C13MACH & SISOCBOT & REGRAS & GLOBAL \\
\hline \hline CADINANO & 78.7 & 61.0 & - & 100.0 & 75.8 \\
EUDESMANO & 6.2 & 19.6 & - & - & 6.7 \\
GIMNOMITRANO & - & - & 50.0 & - & 4.0 \\
MILTAIIANO & - & - & 50.0 & - & 4.0 \\
BICICLOGERMACRANO & - & 9.8 & - & - & 2.4 \\
AROMADENDRANO & - & 9.7 & - & - & 2.3 \\
CAROTANO & 5.8 & - & - & - & 1.9 \\
ESQ11 & 3.6 & - & - & - & 1.2 \\
\hline
\end{tabular}

SUBESQUELETO: $10[4 \mathrm{OH} ; 5 E N]-100.0 \%$ 


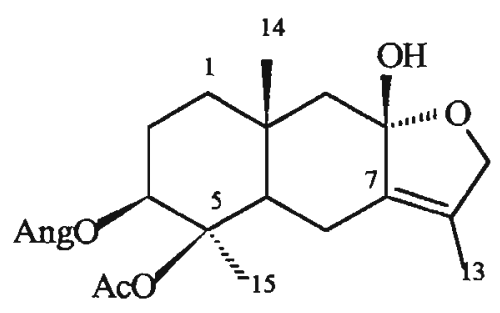

Phytochemistry, 1996, 43, 417

Esqueleto EUDESMANO Pluchea quitoc (Compositae)

$\mathrm{RMN}^{13} \mathrm{C}\left(\mathrm{CDCl}_{3}, \mathrm{C1}-\mathrm{C15}\right): 38,5$ (t); 25,4 (t); 73,8 (d); 87,6 (s); 47,5 (d); 20,9 (t); 129,5 (s); 97,1 (s); 49,7 (t); 35,6 (s); 125,1 (s); 72,9 (t); 13,3 (q); 19,7 (q); 17,0 (q); Ang: 167,0 (s); 128,1 (s); 137,6 (d); 15,7 (q); 20,6 (q); OAc: 22,7 (q); 170,2 (s).

SUBESTRUTURAS PROPOSTAS PELO SISCONST:<smiles>C=C(C)CC(C(C)(C)C)C(C)(C)OC</smiles>

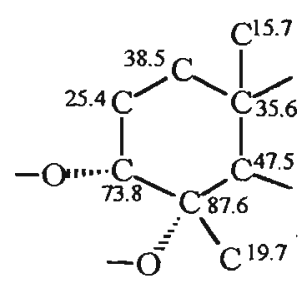

PROBABILIDADE DE ESQUELETO (\%)

\begin{tabular}{lccccc}
\hline \multicolumn{1}{c}{ ESQUELETO } & SISCONST & C13MACH & SISOCBOT & REGRAS & GLOBAL \\
\hline \hline EUDESMANO & 63.7 & 21.0 & 75.0 & 76.9 & 59.1 \\
BICICLOGERMACRANO & - & - & - & 15.4 & 5.5 \\
ARISTOLANO & - & 21.7 & - & - & 5.2 \\
3,4-SECO-13(->)9-DRIMANO & 11.8 & - & - & - & 3.8 \\
CADINANO & 2.8 & 9.8 & - & - & 3.2 \\
4,5-SECO-GUAIANO & - & 9.6 & - & - & 2.3 \\
ESQ48 & - & 9.5 & - & - & 2.3 \\
8,11-SECO-ISHWARANO & -- & 9.5 & - & -- & 2.3 \\
\hline
\end{tabular}

SUBESQUELETO: 1 [7(11)EN] - 76.9\%

MACRONO: Acetato - 170,2s; 20,6q (erro médio=0,800); Angelato - 167,0s; 137,6d; 129,5s; 17,0q $13,3 q$ (erro médio $=1.980$ ). 


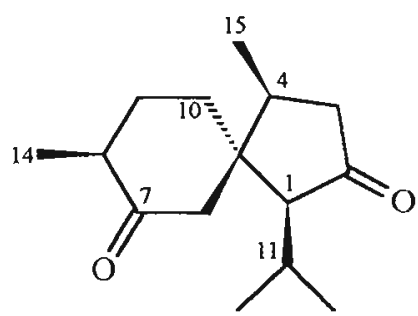

Esqueleto ACORANO
Phytochemistry, 1996, $\underline{43}, 1175$

Acorus calamus (Araceae)

RMN ${ }^{13} \mathrm{C}\left(\mathrm{CDCl}_{3}, \mathrm{C} 1-\mathrm{C} 15\right): 60,3$ (d); 212,5 (s); 40,3 (t); 31,5 (d); 48,1 (s); 45,5 (t); 217,7 (s); 44,4 (d); 29,9 (t); 32,7 (t); 25,0 (d); 24,6 (q); 16,3 (q); 14,3 (q); 19,6 (q).

SUBESTRUTURAS PROPOSTAS PELO PROGRAMA SISCONST:<smiles>CCCCC(C)(C)C(C)C</smiles><smiles>CCC(C)C(C)C(C)C(C)C</smiles>

PROBABILIDADE DE ESQUELETO (\%)

\begin{tabular}{lccccc}
\hline \multicolumn{1}{c}{ ESQUELETO } & SISCONST & C13MACH & SISOCBOT & REGRAS & GLOBAL \\
\hline \hline BULATANO & - & - & - & 100.0 & 36.0 \\
15-NOR-BOURBONANO & 40.1 & - & - & - & 12.8 \\
GUAIANO & - & 9.9 & 100.0 & - & 10.4 \\
AROMADENDRANO & - & 20.4 & - & - & 4.9 \\
CADINANO & - & 20.3 & - & - & 4.9 \\
VETISPIRANO & 8.8 & - & - & - & 2.8 \\
CAROTANO & 8.2 & - & - & - & 2.6 \\
PINGUISANO & - & 10.5 & - & - & 2.5 \\
\hline
\end{tabular}

SUBESQUELETO: 59 [sem substituintes] - 100.0\% 


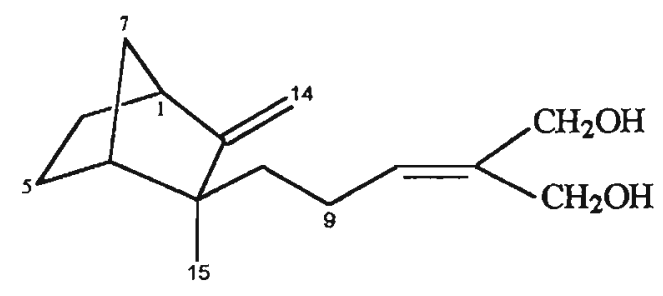

Phytochemistry, 1996, 41,829

Esqueleto $\beta$-SANTALANO

Santalum insulare (Santalaceae)

RMN ${ }^{13} \mathrm{C}\left(\mathrm{CDCl}_{3}, \mathrm{C} 1-\mathrm{C15}\right) ; 46,8$ (d); 166,0 (s); 44,8 (s); 44,7 (d); 23,7 (t); 29,7 (t); 37,1 (t); 41,0 (t); $23,1(\mathrm{t}) ; 131,7(\mathrm{~d}) ; 136,8(\mathrm{~s}) ; 67,6(\mathrm{t}) ; 59,9(\mathrm{t}) ; 99,9(\mathrm{t}) ; 22,7(\mathrm{q})$.

SUBESTRUTURA PROPOSTA PELO SISCONST:

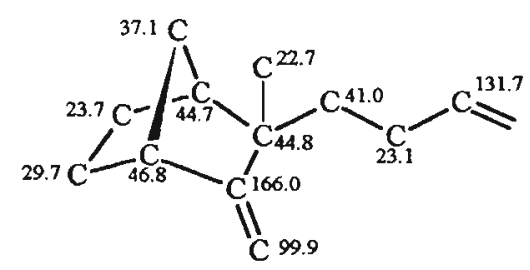

PROBABILIDADE DE ESQUELETO (\%)

\begin{tabular}{lccccc}
\hline \multicolumn{1}{c}{ ESQUELETO } & SISCONST & C13MACH & SISOCBOT & REGRAS & GLOBAL \\
\hline \hline B-SANTALANO & 28.2 & 14.8 & - & 100.0 & 48.6 \\
14(->4) CAROTANO & 26.2 & - & - & - & 8.4 \\
BISABOLANO & - & - & 100.0 & - & 8.0 \\
EUDESMANO & 3.9 & 20.2 & - & - & 6.1 \\
CADINANO & 4.8 & 9.3 & - & - & 3.8 \\
ACORANO & 2.9 & 9.6 & - & - & 3.2 \\
VETISPIRANO & 7.8 & - & - & - & 2.5 \\
15-NOR-BOURBONANO & - & 9.8 & - & - & 2.3 \\
\hline
\end{tabular}

SUBESQUELETO: 126 [sem substituintes] 


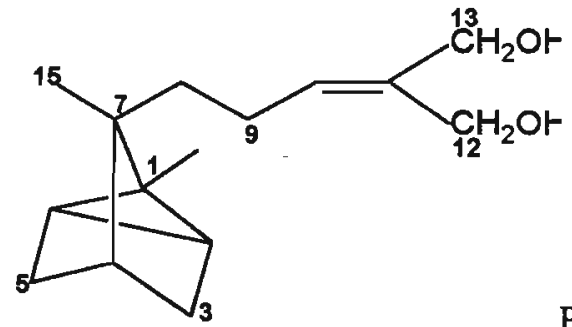

Phytochemistry, 1996, 41, 829

Santalum insulare (Santalaceae)

Esqueleto $\alpha$-SANTALANO

$\mathrm{RMN}^{13} \mathrm{C}\left(\mathrm{CDCl}_{3}, \mathbf{C 1}-\mathrm{C15}\right): 27,4$ (s); 19,6 (d); 31,6 (t); 38,2 (d); 31,1 (t); 19,5 (d); 44,9 (s); 34,6 (t); 22,9 $(\mathrm{t}) ; 132,1(\mathrm{~d}) ; 136,5(\mathrm{~s}) ; 67,4(\mathrm{t}) ; 59,7(\mathrm{t}) ; 10,7$ (q); 17,5 (q).

SUBESTRUTURAS PROPOSTAS PELO SISCONST:
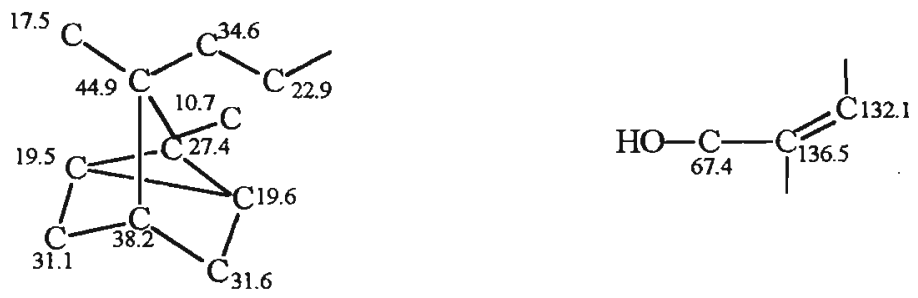

PROBABILIDADE DE ESQUELETO (\%)

\begin{tabular}{lccccc}
\hline \multicolumn{1}{c}{ ESQUELETO } & SISCONST & C13MACH & SISOCBOT & REGRAS & GLOBAL \\
\hline \hline Q-SANTALANO & 86.4 & 100.0 & - & 50.0 & 69.6 \\
PROTOILUDANO & - & - & - & 50.0 & 18.0 \\
BISABOLANO & - & - & 100.0 & - & 8.0 \\
ISHWARANO & 4.9 & - & - & - & 1.6 \\
CICLOMILTAILANO & 4.3 & - & - & - & 1.4 \\
LONGICICLANO & 2.2 & - & - & - & 0.7 \\
OUTROS & 2.2 & & & & 0.7 \\
\hline
\end{tabular}

SUBESQUELETOS: 125 [sem substituintes] - 100.0\%;117[2(4) EN] - 100\% 


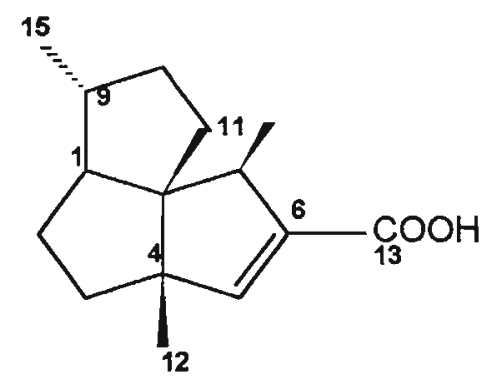

Phytochemistry, 1996, 41, 837

Artemisia Chamaemolifolia (Compositae)

\section{Esqueleto SIFIPERFOLANO}

RMN ${ }^{13} \mathrm{C}\left(\mathrm{CDCl}_{3}, \mathrm{C1}-\mathrm{C15}\right): 64,5$ (d); 30, 0 (t); 37,5 (t); 58,6 (s); 154,3 (d); 138,6 (s); 49,9 (d); 64,0 (s); 43,1 (d); 35,6 (t); 28,9 (t); 19,9 (q); 170,7 (s); 18,0 (q); 21,7(q).

SUBESTRUTURAS PROPOSTAS PELO SISCONST:
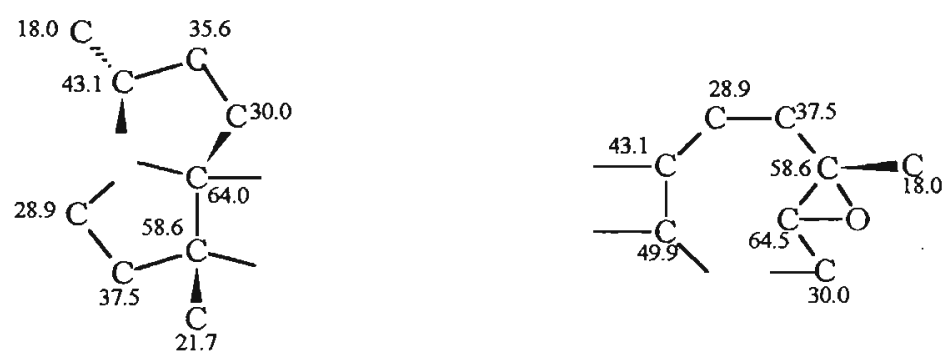

PROBABILIDADE DE ESQUELETO (\%)

\begin{tabular}{lccccc}
\hline \multicolumn{1}{c}{ ESQUELETO } & SISCONST & C13MACH & SISOCBOT & REGRAS & GLOBAL \\
\hline \hline SILFIPERFOLANO & 38.9 & 10.6 & 12.5 & 100.0 & 52.0 \\
CADINANO & 4.0 & 30.0 & 9.4 & - & 9.2 \\
CARIOFILANO & 18.3 & - & - & - & 5.9 \\
BISABOLANO & - & 9.5 & 6.3 & - & 2.8 \\
11-NOR-14 (->3)DRIMANO & - & 10.6 & - & - & 2.5 \\
EREMOFILANO & - & 10.1 & - & - & 2.4 \\
OPLOPANANO & - & 10.0 & - & - & 2.4 \\
AROMADENDRANO & - & 9.6 & - & - & 2.3 \\
OUTROS & - & 7.1 & - & - & 1.7 \\
\hline
\end{tabular}

SUBESQUELETO: $119[130 X 0 ; 13 O R]-100 \%$ 


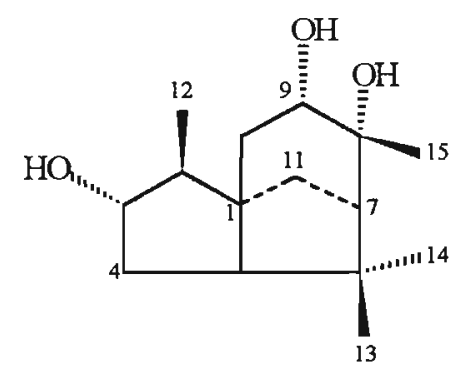

Esqueleto CEDRANO
Phytochemistry, 1996, 42, 1583

Mucor plumbeus (Fungo)

RMN ${ }^{13} \mathrm{C}\left(\mathrm{CDCl}_{3}, \mathrm{C1}\right.$ - C15) : 49,8 (s); 49,5 (d); 80,7 (d); 35,0 (t); 60,4 (d); 40,5 (s); 53,4 (d); 73,8 (s); 70,9 (d); 39,9 (t); 40,5 (t); 12,06 (q);26, 3 (q); 28,0 (q);29,5 (q).

SUBESTRUTURAS PROPOSTAS PELO SISCONST:
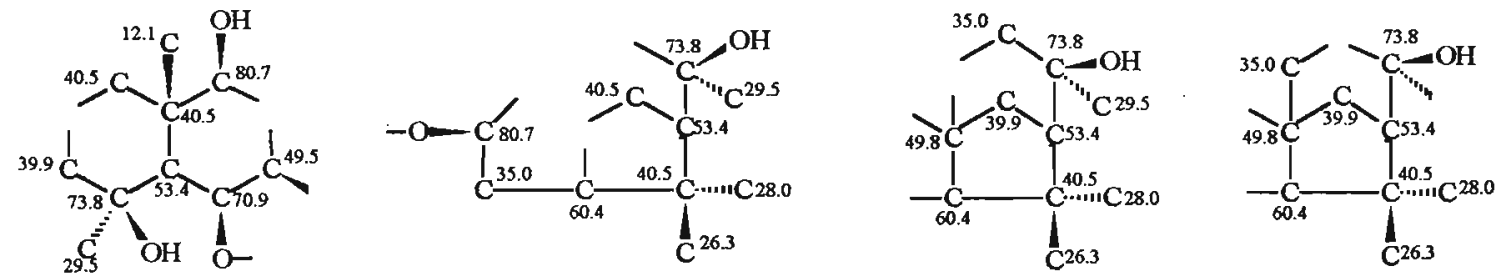

PROBABILIDADE DE ESQUELETO (\%)

\begin{tabular}{lccccc}
\hline ESQUELETO & SISCONST & Cl3MACH & SISOCBOT & REGRAS & GLOBAL \\
\hline \hline 2-10-HIMACHALANO & - & - & - & 50.0 & 18.0 \\
EUDESMANO & 13.2 & 52.3 & - & - & 16.8 \\
PROTOLUDANO & - & - & - & 46.0 & 16.6 \\
CEDRANO & 30.5 & - & - & - & 9.8 \\
HIRSUTANO & 7.2 & - & - & 4.0 & 3.7 \\
CAPNELANO & 10.7 & - & - & - & 3.4 \\
ELEMANO & 1.8 & 10.6 & - & - & 3.1 \\
CAROTANO & 2.8 & 9.1 & - & - & 3.1 \\
\hline
\end{tabular}

SUBESQUELETOS : 92[sem substituintes] -100.0\%; 117[10OR] - 92.0\% 
<smiles>COC(=O)c1ccc2c3c1C(=O)C(=O)C(C)=C3OC=C2C</smiles>

Esqueleto CADINANO

Phytochemistry, 1996, $\underline{43}, 425$

Ulmus davidiana (Ulmaceae)

$\mathbf{R M N}{ }^{13} \mathbf{C}\left(\mathbf{C D C l}_{3}, \mathbf{C 1} 1 \mathbf{C 1 5}\right): 141,9$ (d); 111,7 (s); 132,1 (s); 128,9 (d); 130,3 (d); 137,0 (s); 125,3 (s); $179,0(\mathrm{~s}) ; 178,0$ (s); 114,3 (s); 161,0 (s); 124,2 (s); 12,8 (q); 7,6 (q); 168,6 (s); OMe - 53,4 (q).

SUBESTRUTURA PROPOSTA PELO SISCONST:<smiles>CC=C1C(C)=COC(=C(C)C(C)=O)C1=C(C)C</smiles>

PROBABILIDADE DE ESQUELETO (\%)

\begin{tabular}{lccccc}
\hline ESQUELETO & SISCONST & C13MACH & SISOCBOT & REGRAS & GLOBAL \\
\hline \hline CADINANO & 83.8 & 22.8 & - & 100.0 & 68.3 \\
14(->6)-EREMOFLLANO & 16.2 & - & - & - & 5.2 \\
LAURANO & - & 19.9 & - & - & 4.8 \\
GERMACRANO & -- & 18.9 & - & - & 4.5 \\
EUDESMANO & - & 10.0 & - & - & 2.4 \\
10,11,15-TRINOR-PINGUISANO & - & 9.6 & - & - & 2.3 \\
2,2'-BIS-BISABOLANO & - & 9.4 & - & - & 2.2 \\
ESQ52 & - & 9.3 & - & - & 2.2 \\
\hline
\end{tabular}

SUBESQUELETO : 10 [4EN; $15 \mathrm{OXO} ; 15 \mathrm{OR}]-100.0 \%$ (faixa de erro=0.0); $10[2,3 \mathrm{OXO} ; 5,12 \mathrm{OXI}$; $1(6), 4,7,9 \mathrm{EN}]-100 \%$ (faixa de erro $=1.0$ ). 


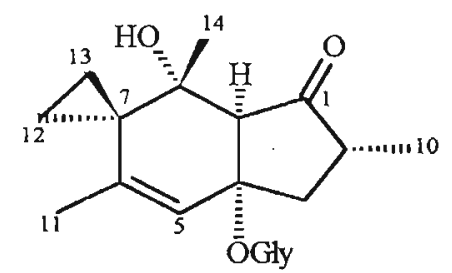

Esqueleto 15-NOR-ILUDANO
Phytochemistry, 1997, $\underline{44}, 901$ Pteridium aquilinum (Pteridaceae)

RMN ${ }^{13}$ C (Piridina-d, C1-C15) : 221,9 (s); 42,5 (t); 43,9 (t); 83,5 (s); 125,2 (d); 138,5 (s); 30,4 (s); 74,2 (s); 61,7 (d); 14,9 (q); 19,5 (q); 7,2 (t); 9,7 (t); 23,0 (q), OGly - 99,9 (d); 75,0 (d); 78,5 (d); 71,9 (d); $79,1(\mathrm{~d}) ; 62,9(\mathrm{t})$.

SUBESTRUTURAS PROPOSTAS PELO SISCONST:
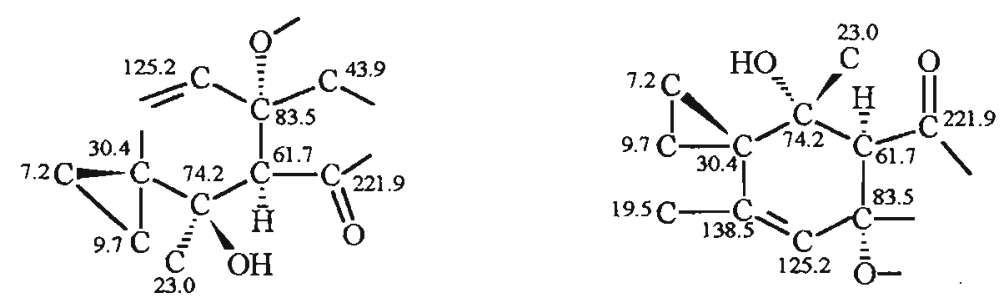

PROBABILIDADE DE ESQUELETO (\%)

\begin{tabular}{lccccc}
\hline ESQUELETO & SISCONST & C13MACH & SISOCBOT & REGRAS & GLOBAL \\
\hline \hline 15-NOR-ILUDANO & 100.0 & 51.7 & - & 100.0 & 80.4 \\
I1-NOR-PTEROSIN & - & - & 61.1 & - & 4.9 \\
CAROTANO & - & 19.2 & - & - & 4.6 \\
PTEROSIN & - & - & 33.3 & - & 2.7 \\
EUDESMANO & - & 9.9 & - & - & 2.4 \\
BISABOLANO & - & 9.8 & - & - & 2.4 \\
HUMULANO & - & 9.4 & - & - & 2.3 \\
\hline
\end{tabular}

OBS: O esqueleto 15-Nor-iludano foi caracterizado no REGRAS apenas pelo seu padrão de substituição.

MACRONO: Glicose - 99,9d; 79,1d; 78,5d; 75,0d; 71,9d; 62,9t (erro médio=1.142); Etóxido - 62,9 t; 14,9q (erro médio $=1.500$ ); Isopropionato $-61,7 \mathrm{~d} ; 23,0 \mathrm{q} ; 19,5 \mathrm{q}$ (erro médio=2.600). 


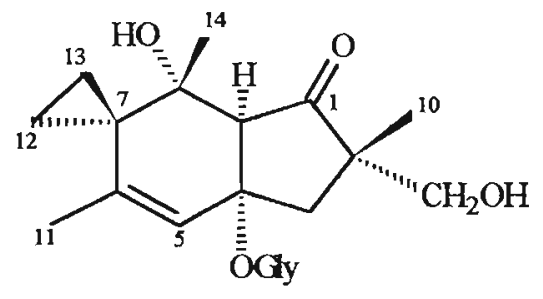

Esqueleto ILUDANO

Phytochemistry, 1997, 44, 901

Pteridium aquilinum (Pteridaceae)

RMN ${ }^{13} \mathrm{C}$ (piridina-d 5, C1-C15) : 225,6 (s); 53,2 (s); 46,1 (t); 81,6 (s); 125,0 (d); 141,7 (s); 29,9 (s); 71,3 (s); 64,2 (d); 21,5 (q); 19,2 (q); 6,2 (t); 9,9 (t); 27,4 (q); 67,8 (t); OGly - 99,6 (d); 75,4 (d); 78,4 (d); $72,1(\mathrm{~d}) ; 78,9(\mathrm{~d}) ; 63,1(\mathrm{t})$.

SUBESTRUTURAS PROPOSTAS PELO SISCONST :
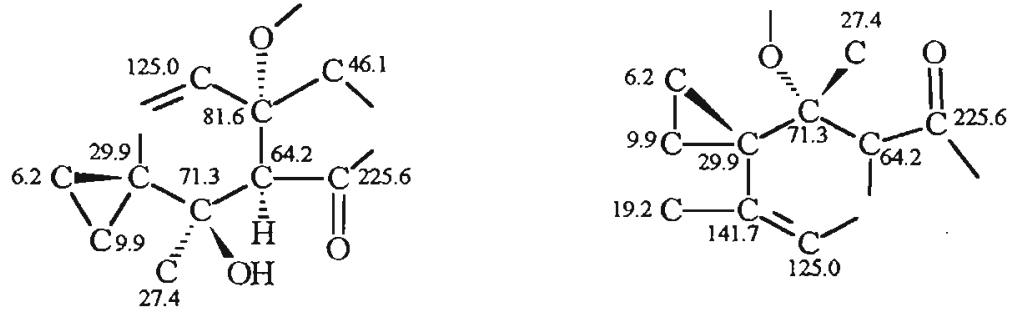

PROBABILIDADE DE ESQUELETO (\%)

\begin{tabular}{lccccc}
\hline ESQUELETO & SISCONST & C13MACH & SISOCBOT & REGRAS & GLOBAL \\
\hline \hline LUDANO & 100.0 & 46.0 & 5.6 & 100.0 & 79.5 \\
11-NOR-PTEROSIN & - & - & 61.1 & - & 4.9 \\
14(->4)-CAROTANO & - & 18.8 & - & - & 4.5 \\
PTEROSIN & - & - & 33.3 & - & 2.7 \\
ESQ14 & - & 9.1 & - & - & 2.2 \\
15(->1)-EUDESMANO & - & 8.7 & - & - & 2.1 \\
CADINANO & - & 8.7 & - & - & 2.1 \\
14-(->6)-EREMOFLANO & - & 8.7 & - & - & 2.1 \\
\hline
\end{tabular}

SUBESQUELETO: 74 [sem substituintes] - 100.0\%

MACRONO: Glicose - 99,6d; 78,9d; 78,4d; 75,4d; 72,1d; 63,1t (erro médio=1.092); Etóxido - 63,1 t; 19,2q (erro médio $=3.650$ ); Isopropionato $-64,2 d ; 21,5 q ; 19,2 q$ (erro médio=2.367). 
<smiles>CC(=O)OC1CC[C@]2(I)CC(=O)C(C(C)(C)O)=CC2C1(C)O</smiles>

Esqueleto EUDESMANO
Phytochemistry, 1996, 43, 417 Pluchea quitoc (Compositae)

RMN ${ }^{13} \mathrm{C}\left(\mathrm{CDCl}_{3}, \mathrm{C1}\right.$ - C15) : 36,5 (t); 25,6 (t); 72,9 (d); 85,7 (s); 48,6 (d); 140,1 (d); 145,1 (s); 200,3 (s); 57,6 (t); 40,0 (s); 71,7 (s); 28,9 (q); 29,1 (q); 18,5 (q); 18,7 (q); Ang-167,0 (s); 127,8 (s); 138,1 (d); 15,7 (q); 20,6 (q); OAc $-22,8$ (q); 170,7 (s).

SUBESTRUTURAS PROPOSTAS PELO SISCONST:
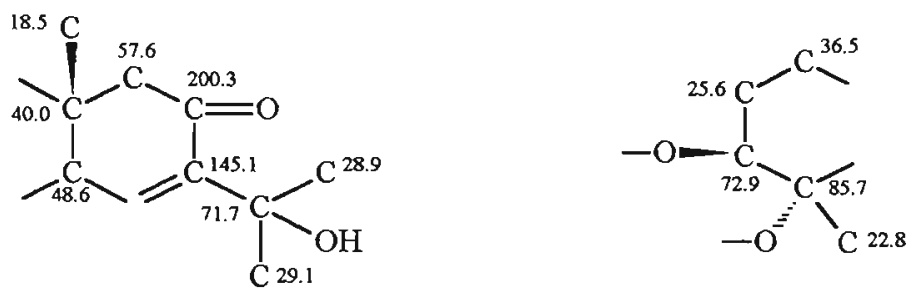

PROBABILIDADE DE ESQUELETO (\%)

\begin{tabular}{lccccc}
\hline ESQUELETO & SISCONST & C13MACH & SISOCBOT & REGRAS & GLOBAL \\
\hline \hline EUDESMANO & 86.2 & 73.3 & 75.0 & 100.0 & 87.2 \\
4,5-SECO-GUAIANO & - & 9.1 & - & - & 2.2 \\
CADINANO & - & 8.9 & - & - & 2.1 \\
HUMULANO & - & 8.7 & - & - & 2.0 \\
NARDOSINANO & 5.3 & - & - & - & 1.7 \\
MONOCICLOFARNESANO & 1.1 & - & 16.7 & - & 1.7 \\
OUTROS & 5.0 & - & - & - & 1.6 \\
CHAMIGRANO & 1.1 & - & -- & -- & 0.4 \\
\hline
\end{tabular}

SUBESQUELETOS: 1 [8OXO] - 100.0\%; 1 [6EN; 8OXO] $-100.0 \%$.

MACRONO: Acetato - 170,7s; 22,8q (erro médio=1.050); Tiglato - 167,0s; 140,1d; 127,8s; 18,7q; 15,7q (erro médio $=1.300$ ). 


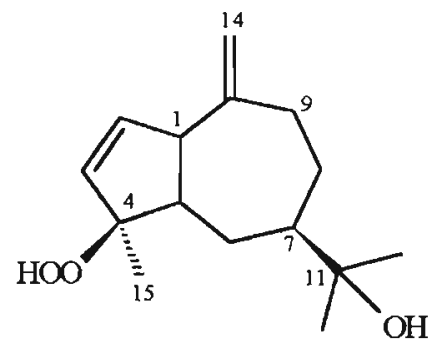

Phytochemistry, 1996, 42,741

Esqueleto GUAIANO Viburnum awabuki (Caprifoliaceae)

RMN ${ }^{13} \mathrm{C}$ (C CD $\left._{6}, \mathbf{C 1}-\mathrm{C} 5\right): 54,1$ (d); 133,6 (d), 136,6 (d); 96,5 (s); 48,8 (d); 27,4 (t); 48,7 (d); 27,3 (t); 39,4 (t); 153,4 (s); 73,9 (s); 26,4 (q); 27,8 (q); 106,9 (t), 19,2 (q).

SUBESTRUTURAS PROPOSTAS PELO SISCONST:

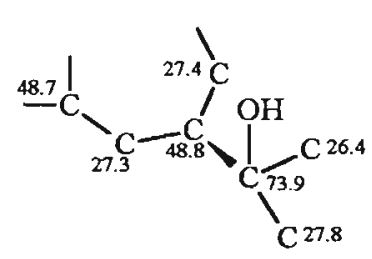

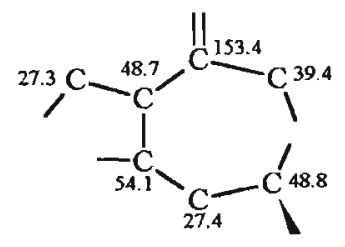<smiles>C=C(C)C(C)CC</smiles>

PROBABILIDADE DE ESQUELETO (\%)

\begin{tabular}{lccccc}
\hline ESQUELETO & SISCONST & C13MACH & SISOCBOT & REGRAS & GLOBAL \\
\hline \hline CADINANO & - & - & - & 100.0 & 36.0 \\
EUDESMANO & 70.4 & 29.8 & - & - & 29.7 \\
GUAIANO & 11.4 & 31.0 & 100.0 & - & 19.1 \\
GERMACRANO & 10.2 & - & - & - & 3.3 \\
AROMADENDRANO & - & 10.8 & - & - & 2.6 \\
ESQ44 & - & 9.6 & - & - & 2.3 \\
LAURANO & - & 9.4 & - & - & 2.2 \\
$9,10-S E C O-D R I M A N O$ & - & 9.3 & - & & 2.2 \\
\hline
\end{tabular}

SUBESQUELETO: 10 [13OR; 4,11EN] - 100.0\% 


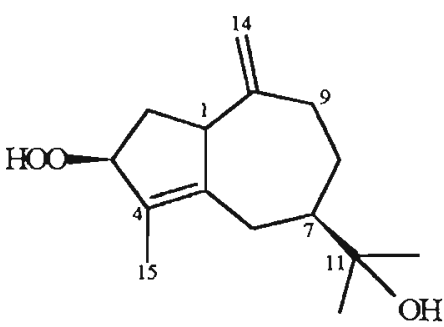

Esqueleto GUAIANO

Phytochemistry, $1996,42,741$ Viburnum awabuki (Caprifoliaceae)

RMN ${ }^{13} \mathrm{C}\left(\mathbf{C}_{6} \mathbf{D}_{6}, \mathbf{C 1}-\mathrm{C} 15\right): 51,3$ (d); 35,8 (t); 92,6 (d); 130,1 (s); 143,4 (s); $29,0(\mathrm{t}) ; 45,8(\mathrm{~d}) ; 30,4$ (t); $34,9(\mathrm{t}) ; 153,1(\mathrm{~s}) ; 72,5(\mathrm{~s}) ; 26,4$ (q); 27,3 (q); 109,6 (t); 19,1 (q).

SUBESTRUTURAS PROPOSTAS PELO SISCONST:

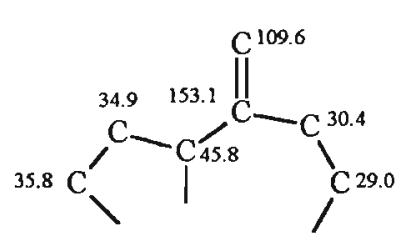<smiles>C=C(CCC)C(C)C(C)CC</smiles><smiles>CCC(CC(C)C)C(C)(C)O</smiles>

PROBABILIDADE DE ESQUELETO (\%)

\begin{tabular}{lccccc}
\hline ESQUELETO & SISCONST & CI3MACH & SISOCBOT & REGRAS & GLOBAL \\
\hline \hline GUAIANO & 51.0 & 20.5 & 100.0 & 100.0 & 65.2 \\
EUDESMANO & 25.6 & 21.2 & - & - & 13.3 \\
CADINANO & 5.6 & 9.6 & - & - & 4.1 \\
BISABOLANO & 1.4 & 9.8 & - & - & 2.8 \\
FITUBERIN & - & 10.1 & - & - & 2.4 \\
ESQ18 & - & 9.6 & - & - & 2.3 \\
ESQ6 & - & 9.6 & - & & 2.3 \\
AROMADENDRANO & - & 9.6 & - & - & 2.2 \\
\hline
\end{tabular}

SUBESQUELETO: $75[11 \mathrm{OH} ; 1(10) \mathrm{EN}]-100.0 \%$ 


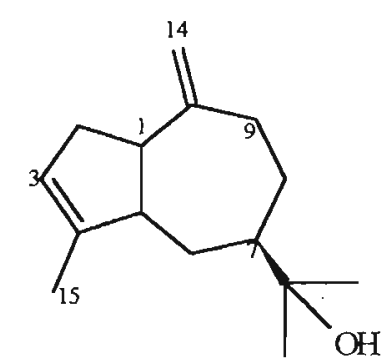

Esqueleto GUAIANO
Phytochemistry, 1996, 42,741 Viburnum awabuki (Caprifoliaceae)

RMN ${ }^{13}$ C (C6 $\left.\mathbf{D}_{6}, \mathbf{C 1}-\mathbf{C 1 5}\right): 49,2$ (d); 34,0 (t); 123,1 (d); 142,0 (s); 51,0 (d); 32,3(t); 48,9 (d); 26,2 (t); $41,1(\mathrm{t}) ; 152,7(\mathrm{~s}) ; 74,1(\mathrm{~s}) ; 25,7(\mathrm{q}) ; 28,0(\mathrm{q}) ; 106,4(\mathrm{t}) ; 14,8(\mathrm{q})$.

SUBESTRUTURAS PROPOSTAS PELO SISCONST:<smiles>C=C(C)C(C)CC(C)C(C)(C)C(C)(C)C</smiles><smiles>CCC(CC)[C@@H](CC)C(C)(C)O</smiles>

$!_{123.1}$<smiles>C=C(C)CC</smiles>

PROBABILIDADE DE ESQUELETO (\%)

\begin{tabular}{lccccc}
\hline ESQUELETO & SISCONST & C13MACH & SISOCBOT & REGRAS & GLOBAL \\
\hline \hline CADINANO & - & - & - & 100.0 & 36.0 \\
GUALANO & 40.3 & 40.0 & 100.0 & - & 30.5 \\
EUDESMANO & 23.5 & 19.9 & - & - & 12.3 \\
OPLOPANANO & 17.6 & - & - & - & 5.6 \\
EREMOFILANO & - & 10.4 & - & - & 2.5 \\
AMBIGUANO & - & 10.2 & - & - & 2.5 \\
FARNESANO & - & 9.9 & - & - & 2.4 \\
AROMADENDRANO & - & 9.7 & - & - & 2.3 \\
\hline
\end{tabular}

SUBESQUELETO: $10[110 \mathrm{OH}]-100.0 \% ; 10[8,9 \mathrm{OR} ; 10(14) \mathrm{EN}]-100.0 \%$ 


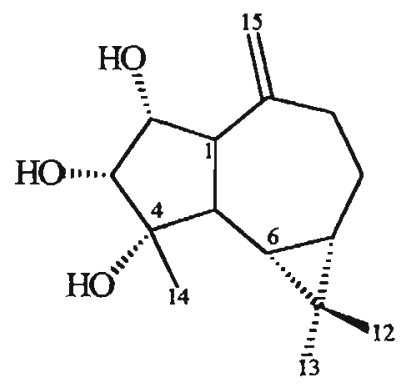

Phytochemistry, 1996, $\underline{43}, 83$

Esqueleto AROMADENDRANO Heteroscyphus planus (Jungermanniaceae)

$\mathbf{R M N}{ }^{13} \mathbf{C}\left(\mathrm{CDCl}_{3}, \mathbf{C 1}-\mathrm{C15}\right): 53,2$ (d); 74,2 (d); 76,1 (d); 81,4 (s); 45,9 (d); 26,1 (d); 25,2 (d); 21,1 (t); $37,4(\mathrm{t}) ; 147,9$ (s); 17,7 (s); 28,4 (q); 15,9 (q); 22,3 (q); 110,2 (t).

SUBESTRUTURA PROPOSTA PELO SISCONST:

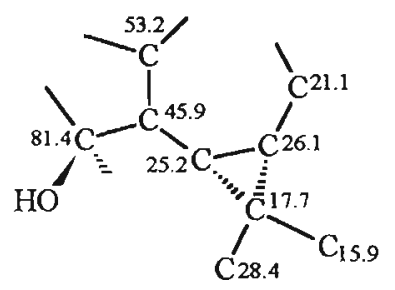

PROBABILIDADE DE ESQUELETO (\%)

\begin{tabular}{lccccc}
\hline ESQUELETO & SISCONST & C13MACH & SISOCBOT & REGRAS & GLOBAL \\
\hline \hline AROMADENDRANO & 71.8 & 60.6 & 3.8 & 100.0 & 73.8 \\
EUDESMANO & 11.2 & 9.9 & - & - & 6.0 \\
GUAIANO & 1.6 & 9.5 & - & - & 2.8 \\
CADINANO & 4.1 & - & 19.2 & - & 2.8 \\
FARNESANO & - & 10.4 & - & - & 2.5 \\
MONOCICLOFARNESANO & - & 9.7 & - & - & 2.3 \\
2,3SECO-AROMADENDRANO & 5.8 & - & - & - & 1.9 \\
OPLOPANANO & 3.0 & - & - & - & 1.0 \\
\hline
\end{tabular}

SUBESQUELETO : 87 [sem substituintes] - 90.7\%; 87[10(14)EN] - $100.0 \%$ 


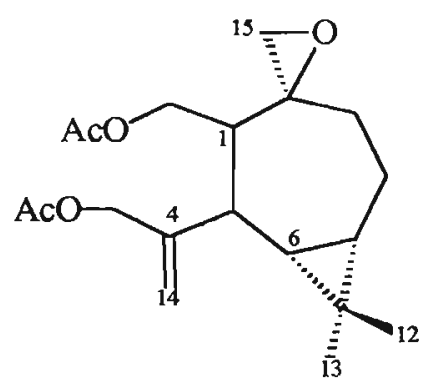

Esqueleto 2,3-SECO-AROMADENDRANO
Phytochemistry, 1996, 43,83 Heteroscyphus planus (Jungermanniaceae)

RMN ${ }^{13} \mathrm{C}\left(\mathrm{CDCl}_{3}, \mathrm{C1}-\mathrm{C15}\right): 47,1$ (d); 62,6 (t); 66,5 (t); 145,1 (s); 38,0 (d); 25,4 (d); 26,1 (d); 21,3 (t); $33,0(\mathrm{t}) ; 62,5$ (s); 18,5 (s); 28,6 (q); 14,4 (q);114,1 (t); 52,9 (t); OAc : 170,5 (s); 171,2 (s); 21,0 (q); 21,0 (q).

SUBESTRUTURAS PROPOSTAS PELO SISCONST:

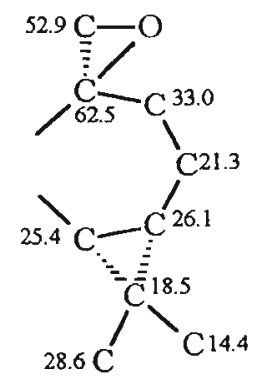

PROBABILIDADE DE ESQUELETO (\%)

\begin{tabular}{lccccc}
\hline ESQUELETO & SISCONST & C13MACH & SISOCBOT & REGRAS & GLOBAL \\
\hline \hline 2,3-SECO-AROMADENDRANO & 70.2 & 40.8 & - & 100.0 & 68.3 \\
AROMADENDRANO & 29.8 & 30.1 & 3.8 & - & 17.1 \\
TREMULANO & - & 9.8 & - & - & 2.4 \\
11,12,13 -TRINOR-ELEMANO & - & 9.8 & - & - & 2.4 \\
13-NOR-ELEMANO & - & 9.5 & - & - & 2.3 \\
ARISTOLANO & - & - & 23.1 & - & 1.8 \\
CADINANO & - & - & 19.2 & - & 1.5 \\
CUPARANO & - & - & 15.4 & - & 1.2 \\
\hline
\end{tabular}

OBS: O padrão de substituição do esqueleto 2,3-Seco-aromadendrano foi suficiente para caracterizá-lo. MACRONO: Acetato - 170,5s; 21,0q (erro médio=0.750); Acetato - 171,2s; 21,0q (erro médio $=1.100$ ). 
<smiles>C=C1CC[C@H]2[C@@H](C)[C@@H](OC(C)=O)[C@H]2C1CO</smiles>

Esqueleto 2,3-SECO-AROMADENDRANO
Phytochemistry, 1996, 43, 83

Heteroscyphus planus (Jungermanniaceae)

RMN ${ }^{13}$ C (CDCl, C1-C15): 51,6 (d); 91,4 (d); 137,8 (d); 120,8 (s); 33,4 (d); 29,6 (d); 29,3 (d); 25,6 (t); $35,2(\mathrm{t}) ; 148,5$ (s); 19,6 (s); 28,8 (q); 15,8 (q); 61,7 (t); 116,3 (t); OAc : 169,8 (s); 21,0 (q).

SUBESTRUTURAS PROPOSTAS PELO SISCONST:<smiles>C=C1CCC2C([C@H](C)[C@@H]2C(OC)(OC)OC)C1(C)C</smiles><smiles>CO/C=C(\C)COC</smiles>

PROBABILIDADE DE ESQUELETO (\%)

\begin{tabular}{lccccc}
\hline ESQUELETO & SISCONST & Cl3MACH & SISOCBOT & REGRAS & GLOBAL \\
\hline \hline 2,3-SECO-AROMADENDRANO & 91.2 & 83.1 & - & 100.0 & 85.1 \\
AROMADENDRANO & 8.0 & 16.9 & 3.8 & - & 6.9 \\
ARISTOLANO & - & - & 23.1 & - & 1.8 \\
CADINANO & - & - & 19.2 & - & 1.5 \\
CUPARANO & - & - & 15.4 & - & 1.2 \\
CHIIOSCIFANO & - & - & 7.7 & - & 0.6 \\
OUTROS & 0.8 & - & - & - & 0.3 \\
BISABOLANO & - & - & 3.8 & - & 0.3 \\
\hline
\end{tabular}

MACRONO: Acetato - 169,8s; 21,0q (erro médio $=0.400$ ). 


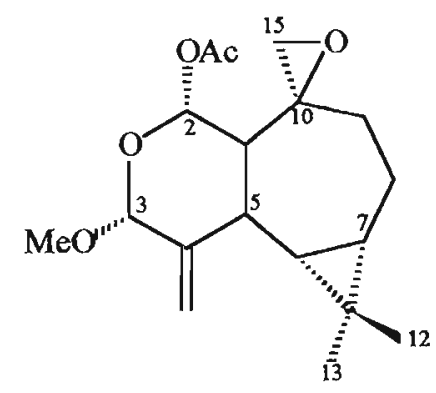

Phytochemistry, 1996, 43,83

Esqueleto 2,3-SECO-AROMADENDRANO Heteroscyphus planus (Jungermanniaceae)

RMN ${ }^{13} \mathrm{C}$ (CDCl 3 , C1-C15): 51,6 (d); 91,4 (d); 137,8 (d); 120,8 (s); 33,4 (d); 29,6 (d); 29,3 (d); 25,6 (t); 35,2 (t); 148,5 (s); 19,6 (s); 28,8 (q); 15,8 (q); 61,7 (t);116,3 (t); OAc - 170,6 (s); 21,4 (q); OMe - 52,2 (q).

SUBESTRUTURAS PROPOSTAS PELO SISCONST:

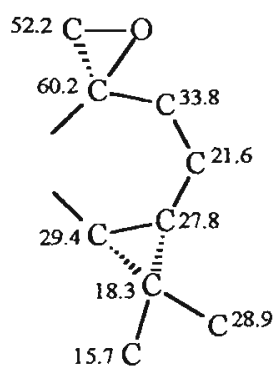<smiles>C=CC(C)C(C(=C)C)C(C)C</smiles>

PROBABILIDADE DE ESQUELETO (\%)

\begin{tabular}{lccccc}
\hline ESQUELETO & SISCONST & C13MACH & SISOCBOT & REGRAS & GLOBAL \\
\hline \hline 2,3-SECO-AROMADENDRANO & 75.3 & 73.6 & - & 100.0 & 77.8 \\
AROMADENDRANO & 21.9 & 17.7 & 3.8 & - & 11.6 \\
ARISTOLANO & - & 8.7 & 23.1 & - & 3.9 \\
CADINANO & 2.6 & - & 19.2 & - & 2.4 \\
CUPARANO & - & - & 15.4 & - & 1.2 \\
CHIIISCIFANO & - & - & 7.7 & - & 0.6 \\
BISABOLANO & 0.8 & - & 3.8 & - & 0.6 \\
TRICOTECANO & - & - & 3.8 & - & 0.3 \\
\hline
\end{tabular}

MACRONO: Acetato - 170,6s; 21,4q (erro médio $=1.000$ ); Metóxi $-55,5 \mathrm{~s}($ erro médio $=2.500$ ). 
<smiles>C=C(C(=O)O)C1CC[C@@]2(C)CCC(O)C(=C)[C@]2(O)C1</smiles>

Phytochemistry, 1997, 44, 897

Esqueleto EUDESMANO Tessaria absinthioides (Compositae)

RMN ${ }^{13} \mathbf{C}\left(\mathbf{C D C l}_{3}, \mathbf{C 1}-\mathbf{C 1 5}\right): 30,2(\mathrm{t}) ; 28,2(\mathrm{t}) ; 74,9$ (d); 146,9 (s); 76,9 (s); 36,6 (t); 36,0 (d); 25,6 (t); $33,0(\mathrm{t}) ; 38,6(\mathrm{~s}) ; 144,4(\mathrm{~s}) ; 171,3(\mathrm{~s}) ; 124,2(\mathrm{t}) ; 116,4(\mathrm{t}) ; 22,3$ (q).

SUBESTRUTURAS PROPOSTAS PELO SISCONST:
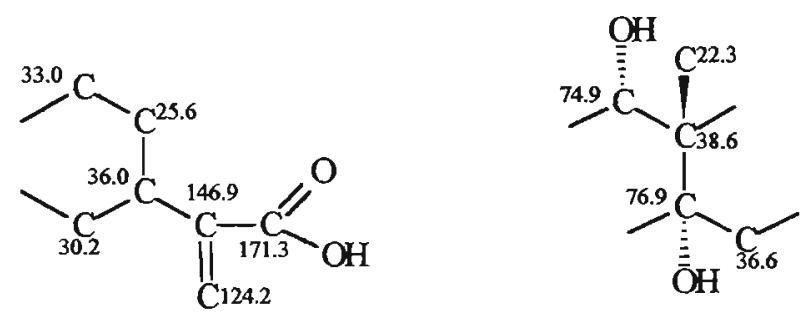

PROBABILIDADE DE ESQUELETO (\%)

\begin{tabular}{lccccc}
\hline ESQUELETO & SISCONST & C13MACH & SISOCBOT & REGRAS & GLOBAL \\
\hline \hline EUDESMANO & 57.8 & 30.6 & 100.0 & 94.0 & 67.7 \\
EREMOFILANO & 15.6 & - & - & - & 5.0 \\
BISABOLANO & 2.4 & 11.1 & - & - & 3.4 \\
DRIMANO & 1.9 & 9.8 & - & - & 3.0 \\
ARISTOLANO & 1.4 & 9.6 & - & - & 2.8 \\
PSEUDOGUAIANO & - & 10.3 & - & - & 2.5 \\
CADINANO & - & 9.6 & - & - & 2.3 \\
HUMULANO & - & 9.5 & - & - & 2.3 \\
\hline
\end{tabular}

SUBESQUELETO: $1[13 \mathrm{OXO}, 13 \mathrm{OR} ; 11 \mathrm{EN}]-94.0 \%$ 
<smiles>CC1=C2C(=O)C(C(C)C)CC[C@]2(C)[C@H](O)C[C@H]1O</smiles>

Esqueleto EUDESMANO
Phytochemistry, 1996, 43, 1175 Acorus Calamus (Araceae)

RMN ${ }^{13} \mathrm{C}\left(\mathrm{CDCl}_{3}, \mathrm{C1}-\mathrm{C15}\right): 72,9$ (d); 35,9 (t); 69,9 (d); 142,5 (s); 133,7 (s); 207,8 (s); 58,2 (d); 22,6 (t); 37,0 (t); 44,0 (s); 25,8 (d); 17,8 (q); 21,1 (q); 16,3 (q); 18,4 (q).

SUBESTRUTURAS PROPOSTAS PELO SISCONST:<smiles>C=C1C[C@H](O)[C@H](Br)[C@](C)(Cl)C1</smiles><smiles>CC1=CC(C)(C)CCC1=O</smiles><smiles>C[C@@H](CCC(C)(C)C)C(C)(C)C</smiles>

PROBABILIDADE DE ESQUELETO (\%)

\begin{tabular}{lccccc}
\hline ESQUELETO & SISCONST & C13MACH & SISOCBOT & REGRAS & GLOBAL \\
\hline \hline CAROTANO & 57.6 & 40.6 & - & 41.7 & 43.2 \\
EUDESMANO & 13.0 & - & - & 38.4 & 18.0 \\
GUAIANO & - & - & 100.0 & - & 8.0 \\
SLFIPERFOLANO & - & 20.0 & - & - & 4.8 \\
ACORANO & - & - & - & 8.4 & 3.0 \\
BICICLOGERMACRANO & - & - & - & 7.7 & 2.8 \\
EREMOFILANO & 8.7 & - & - & & 2.8 \\
BISABOLANO & - & 10.1 & - & - & 2.4 \\
\hline
\end{tabular}

SUBESQUELETOS: $1[7(11) E N]-76.9 \% ; 79[6(10) E N]-83.3 \%$. 
<smiles>CC(C)C1CCC(=O)c2cc(O)c(I)cc21</smiles>

Esqueleto CADINANO
Fitoterapia, 1997, $\underline{68}, 87$ Trichilia catigua (Meliaceae)

$\mathbf{R M N}{ }^{13} \mathbf{C}\left(\mathrm{CDCl}_{3}, \mathbf{C 1 - C 1 4 )}: 139,9\right.$ (s); 112,1 (d); 153,1 (s); 131,3 (s); 131,3 (s); 44,0 (d); 24,4 (t); 35,3 (t); 199,1 (s); 30,2 (d); 19,7 (q); 21,6 (q); 16,5 (q).

\section{SUBESTRUTURAS PROPOSTAS PELO SISCONST:}
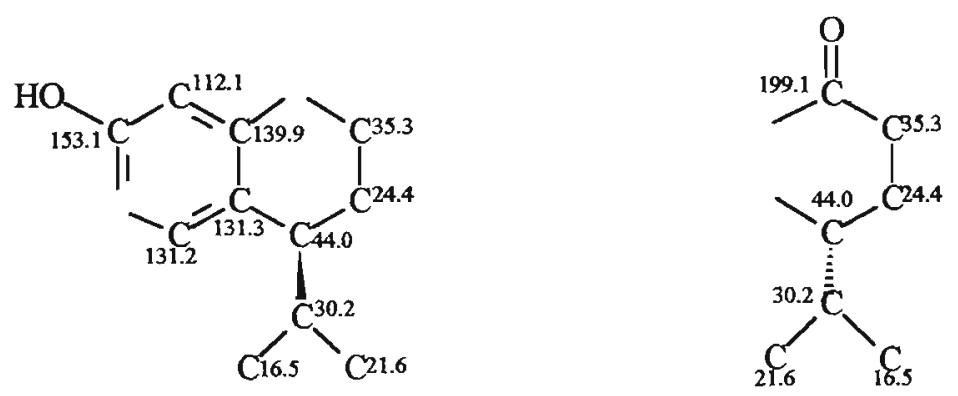

PROBABLIDADE DE ESQUELETO (\%)

\begin{tabular}{lccccc}
\hline ESQUELETO & SISCONST & C13MACH & SISOCBOT & REGRAS & GLOBAL \\
\hline \hline CADINANO & 32.0 & 29.7 & 50.0 & - & 21.4 \\
BISABOLANO & 29.0 & 30.1 & - & - & 16.5 \\
1,10-SECO-CADINANO & - & 10.7 & - & - & 2.6 \\
14-NOR-EREMOFLANO & - & - & - & 20.0 & 7.2 \\
14-NOR-CADINANO & - & - & - & 20.0 & 7.2 \\
12-NOR-CARIOFILANO & - & - & - & 20.0 & 7.2 \\
15-NOR-EUDESMANO & - & - & - & 20.0 & 7.2 \\
14-NOR-GUAIANO & - & - & - & 20.0 & 7.2 \\
\hline
\end{tabular}




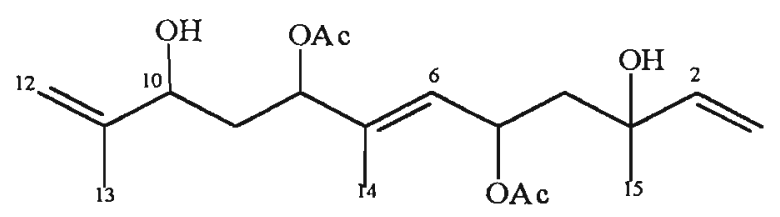

Esqueleto FARNESANO

Phytochemistry, 1997, 44, 311 Tanacetum aucheranum (Compositae)

$\mathbf{R M N}{ }^{13} \mathbf{C}\left(\mathbf{C D C l}_{3}, \mathbf{C 1}-\mathbf{C 1 5}\right): 112,2$ (t); 114,2 (d); 72,4 (s); 39,0 (t); 68,7 (d); 125,9 (d); 137,2 (s); 75,3 (d); 46,3 (t); 71,6 (d); 146,8 (s); 111,0 (t); 17,9 (q);13,2 (q);28,4 (q); OAc - 170,8 (s);21,2 (q); 170,1 (s); 21,4 (q).

SUBESTRUTURAS PROPOSTAS PELO SISCONST:
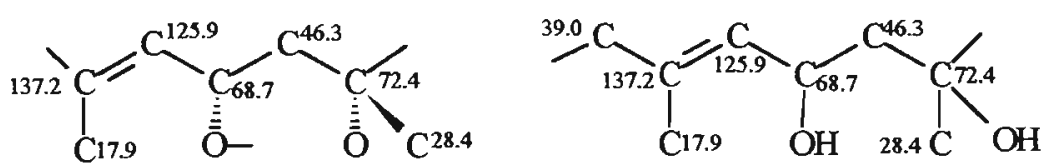<smiles>C=C(C)[C@@H](O)[C@@H](C)O</smiles>

PROBABILIDADE DE ESQUELETO (\%)

\begin{tabular}{lccccc}
\hline ESQUELETO & SISCONST & CI3MACH & SISOCBOT & REGRAS & GLOBAL \\
\hline \hline FARNESANO & 26.4 & 61.6 & 55.6 & 100.0 & 63.7 \\
TRICOTECANO & 41.4 & - & - & - & 13.2 \\
GERMACRANO & 20.9 & 9.6 & 22.2 & - & 10.8 \\
ELEMANO & - & 19.4 & - & - & 4.7 \\
4,5-SECO-GUAIANO & - & 9.5 & - & - & 2.3 \\
MONOCICLOFARNESANO & 5.0 & - & - & - & 1.6 \\
LONGIPINANO & - & - & 11.1 & - & 0.9 \\
ESQ23 & 1.8 & - & - & - & 0.6 \\
\hline
\end{tabular}

SUBESQUELETO: $134[2,6,10 E N]-100.0 \%$. 


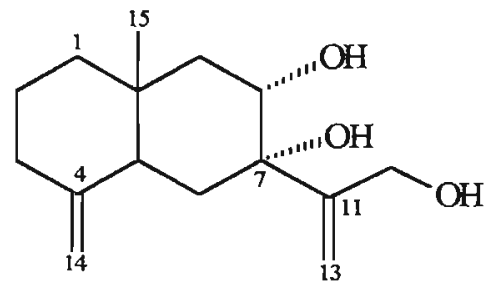

Phamnazie, 1997, 52, 245

Esqueleto EUDESMANO

Rhaponticum uniflorum (Compositae)

$\mathbf{R M N}{ }^{13} \mathbf{C}\left(\mathbf{C D C l}_{3}, \mathbf{C 1}-\mathbf{C 1 5}\right): 36,0(\mathrm{t}) ; 22,9(\mathrm{t}) ; 37,0(\mathrm{t}) ; 149,6(\mathrm{~s}) ; 43,3(\mathrm{~s}) ; 41,2(\mathrm{t}) ; 77,1(\mathrm{~s}) ; 70,2$ $(d) ; 43,4(t) ; 37,0(s) ; 153,4(s) ; 64,6(t) ; 114,7(t) ; 105,5(t) ; 16,6(q)$.

SUBESTRUTURAS PROPOSTAS PELO SISCONST:
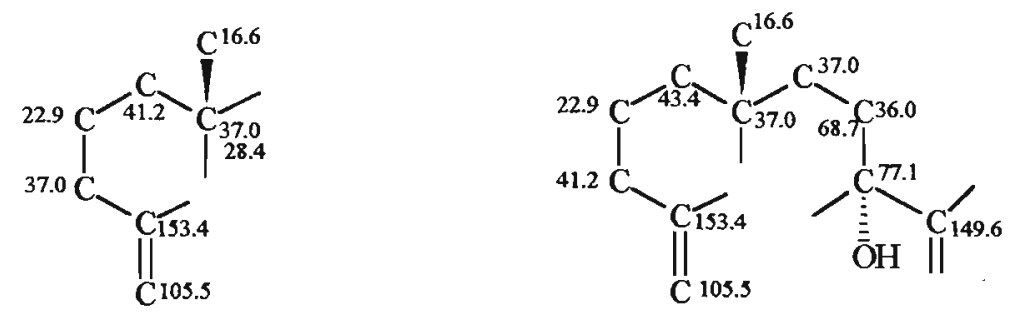

PROBABILIDADE DE ESQUELETO (\%)

\begin{tabular}{lccccc}
\hline ESQUELETO & SISCONST & C13MACH & SISOCBOT & REGRAS & GLOBAL \\
\hline \hline EUDESMANO & 68.4 & 71.4 & 100.0 & - & 47.0 \\
VETISPIRANO & - & - & - & 100.0 & 36.0 \\
CAROTANO & 15.8 & - & - & - & 5.1 \\
IRIFARANO & 10.5 & - & - & - & 3.4 \\
EREMOFILANO & 2.6 & 9.1 & - & - & 3.0 \\
BISABOLANO & - & 10.1 & - & - & 2.4 \\
NEOLEMNANO & - & 9.4 & - & - & 2.3 \\
DRIMANO & 2.6 & - & - & - & 0.8 \\
\hline
\end{tabular}

SUBESQUELETO: $73[2 \mathrm{OR} ; 14 \mathrm{OR}]-100.0 \%$ 
<smiles>CC1=C[C@H](O)C([C@@](C)(O)C/C=C\C(C)(C)O)CC1=O</smiles>

Esqueleto BISABOLANO Achilla cretica (Compositae)

RMN ${ }^{13} \mathrm{C}\left(\mathbf{C D C l}_{3}, \mathbf{C 1}\right.$ - C15) : 64,9 (d); 142,7 (d); 137,3 (s); 200,3 (s); 33,3 (t); 44,1 (d); 74,3 (s); 42,7 $(\mathrm{t}) ; 120,8$ (d); 141,7 (d); 70,7 (s); 29,8 (q);29,8 (q);25,3 (q); 15,8 (q).

SUBESTRUTURAS PROPOSTAS PELO SISCONST:<smiles>CC(C)(O)CC=CC(C)(O)C(C)(O)C(C)(C)C</smiles><smiles>CC1=CC[C@@H](C(C)(C)O)[C@H](C)C1=O</smiles>

PROBABILIDADE DE ESQUELETO (\%)

\begin{tabular}{lccccc}
\hline ESQUELETO & SISCONST & C13MACH & SISOCBOT & REGRAS & GLOBAL \\
\hline \hline BISABOLANO & 28.9 & 42.1 & - & 100.0 & 55.4 \\
FARNESANO & 24.4 & 30.0 & 42.1 & - & 18.4 \\
MONOCICLOFARNESANO & 14.4 & - & - & - & 4.6 \\
EUDESMANO & 4.4 & 9.2 & - & - & 3.6 \\
PACIFIGORGIANO & 8.9 & - & - & - & 2.9 \\
1,2-DINOR-FARNESANO & 1.1 & 9.6 & - & - & 2.7 \\
LACTARANO & - & 9.1 & - & - & 2.2 \\
GUAIANO & 5.6 & - & - & - & 1.8 \\
\hline
\end{tabular}

SUBESQUELETO: $\quad 129[7,110 X \mathrm{XI}]-100.0 \%$ 
<smiles>CC(=O)OCCCC(C)[C@@H]1CC=C(C)C(=O)C1</smiles>

Esqueleto BISABOLANO
Phytochemistry, 1996, 42, 737 Achillea cretica (Compositae)

RMN ${ }^{13} \mathrm{C}\left(\mathrm{CDCl}_{3}, \mathbf{C 1}-\mathrm{C15}\right): 27,3$ (t); 144,6 (d); 135,4 (s); 199,1 (s); 39,2 (t); 44,6 (t); 73,1 (s). 39,1 (t); 25,3 (t); 128,8 (d); 130,7 (s); 13,9 (q); 69,9 (t);24,4 (q); 15,5 (q); OAc - 170,8 (s); 20,7 (q).

SUBESTRUTURAS PROPOSTAS PELO SISCONST:
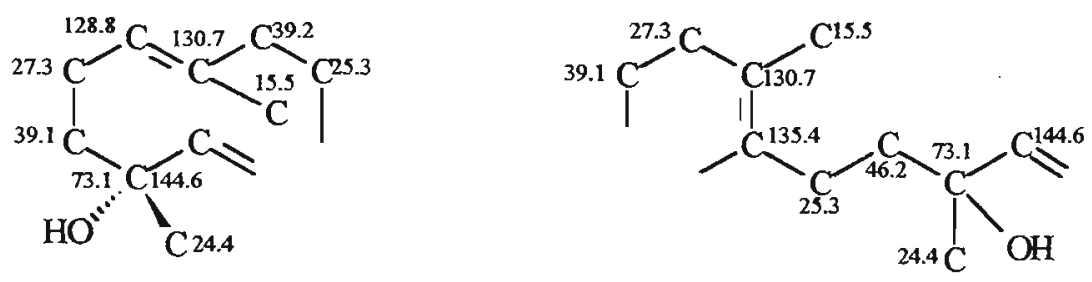

PROBABILIDADE DE ESQUELETO (\%)

\begin{tabular}{|c|c|c|c|c|c|}
\hline ESQUELETO & SISCONST & Cl3MACH & SISOCBOT & REGRAS & GLOBAL \\
\hline BISABOLANO & - & 19.0 & - - & 50.0 & 222.6 \\
\hline CADINANO & - & - & - & 50.0 & 18.0 \\
\hline FARNESANO & - & 51.5 & 42.1 & - & 15.7 \\
\hline 15(->9)-MODEFANO & - & 19.9 & - & - & 4.8 \\
\hline DRIMANO & - & - & 42.1 & - & 3.4 \\
\hline 13,14-DINOR-DRIMANO & - & 9.6 & - & - & 2.3 \\
\hline 3,4-SECO-13(->9)-DRIMANO & - & - & 10.5 & - & 0.8 \\
\hline 13(->9)-DRIMANO & - & - & 5.3 & - & 0.4 \\
\hline
\end{tabular}

SUBESQUELETO: $10[3 \mathrm{OXO} ; 4 \mathrm{EN}]-100.0 \% ; \quad 129[7,11 \mathrm{OXI}]-100.0 \%$. 
<smiles>CC(=O)/C=C/C[C@](C)(O)C1CC=C(C)C(=O)C1</smiles>

Esqueleto 13-NOR- BISABOLANO
Phytochemistry, 1996, $\underline{42}, 737$ Achillea cretica (Compositae)

RMN ${ }^{13} \mathrm{C}\left(\mathrm{CDCl}_{3}, \mathrm{C1}-\mathrm{C14}\right): 27,1(\mathrm{t}) ; 144,1$ (d); 135,5 (s); 199,3 (s); 38,9 (t); 44,8 (d); 73,1 (s); 42,8 (t); 142,3 (d); 134,3 (d); 197,7 (s); 29,7 (q); 24,4 (q); 15,4 (q).

SUBESTRUTURAS PROPOSTAS PELO SISCONST:
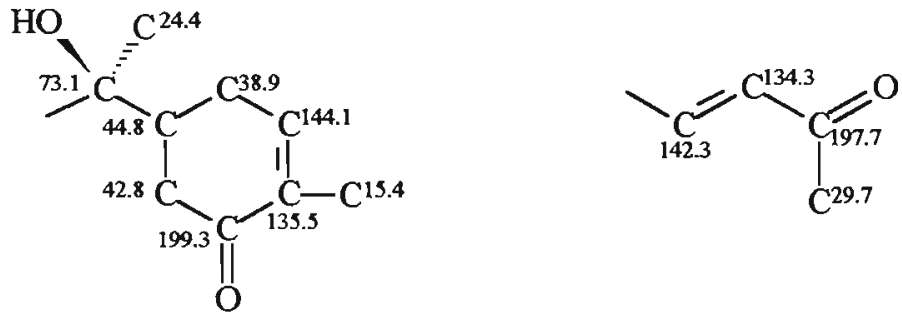

PROBABILIDADE DE ESQUELETO (\%)

\begin{tabular}{lccccc}
\hline ESQUELETO & SISCONST & C13MACH & SISOCBOT & REGRAS & GLOBAL \\
\hline \hline BISABOLANO & 23.3 & 51.9 & - & - & 19.9 \\
13-NOR-BISABOLANO & - & - & - & 50.0 & 18.0 \\
ESQ33 & - & - & - & 50.0 & 18.0 \\
FARNESANO & 16.7 & 9.4 & 42.1 & - & 11.0 \\
GERMACRANO & 29.9 & - & - & - & 9.6 \\
15(->9)-MODEFANO & - & 19.1 & - & - & 4.6 \\
MODEFANO & 1.4 & 10.1 & - & - & 2.9 \\
CADINANO & 8.7 & - & - & - & 2.8 \\
\hline
\end{tabular}




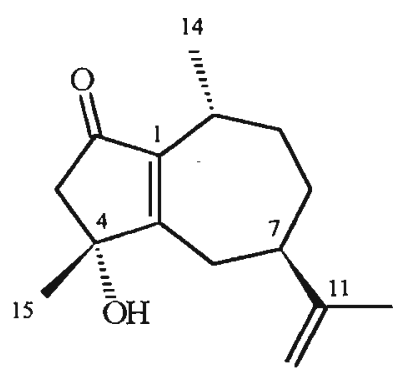

Esqueleto GUAIANO
Phytochemistry, 1997, 45, 149 Joannesia princeps (Euphorbiaceae)

$\mathbf{R M N}{ }^{13} \mathrm{C}\left(\mathrm{CDCl}_{3}, \mathrm{C} 1\right.$ - C15) : 145,5 (s); 204,4 (s); 51,4 (t); 75,8 (s); 174,4 (s); 28,3 (t); 44,5 (d); 28,9 (t); 28,9 (t); 28,3 (d); 149,6 (s); 109,6 (t); 20,1 (q);17,7 (q);26,5 (q).

\section{SUBESTRUTURAS PROPOSTAS PELO SISCONST:}<smiles></smiles>

PROBABILIDADE DE ESQUELETO (\%)

\begin{tabular}{lccccc}
\hline ESQUELETO & SISCONST & CI3MACH & SISOCBOT & REGRAS & GLOBAL \\
\hline \hline GUAIANO & 81.0 & 14.9 & - & 100.0 & 65.5 \\
GIMNOMITRANO & - & - & 100.0 & - & 8.0 \\
CAROTANO & - & 24.6 & - & - & 5.9 \\
CADINANO & 14.3 & - & - & - & 4.6 \\
EUDESMANO & 4.8 & 10.1 & - & - & 4.0 \\
GERMACRANO & - & 10.7 & - & - & 2.6 \\
PRE-SEICHELAANO & - & 5.7 & - & - & 1.4 \\
PINGUISANO & - & 4.9 & - & - & 1.2 \\
\hline
\end{tabular}

SUBESQUELETO: $75[3 \mathrm{OXO} ; 4,11 \mathrm{EN}]-100.0 \%$ 


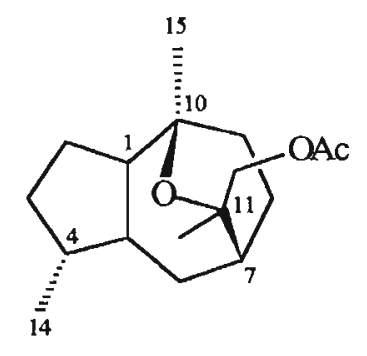

Esqueleto GUAIANO
Phytochemistry, 1996, $\underline{41}, 977$

Valeriana officialis (Valerianaceae)

$\mathbf{R M N}^{13} \mathbf{C}\left(\mathrm{CDCl}_{3}, \mathbf{C 1}-\mathbf{C 1 5}\right): 50,3(\mathrm{~d}) ; 28,2(\mathrm{t}) ; 32,0(\mathrm{t}) ; 32,8$ (d); 41,6 (d); 33,0 (t); 31,4 (d); 23,7 (t); $35,0(\mathrm{t}) ; 74,5$ (s); 75,5 (s); 70,6 (t); 23,4 (q); 18,4 (q);27,4 (q); OAc - 21,0 (q); 171,0 (s).

SUBESTRUTURA PROPOSTA PELO SISCONST:

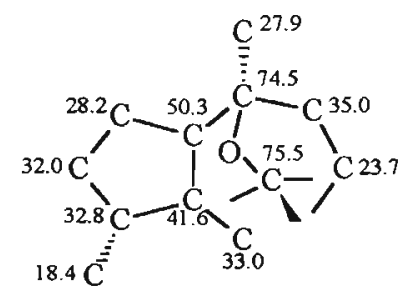

PROBABLIDADE DE ESQUELETO (\%)

\begin{tabular}{lccccc}
\hline ESQUELETO & SISCONST & C13MACH & SISOCBOT & REGRAS & GLOBAL \\
\hline \hline CADINANO & 33.3 & - & - & 100.0 & 46.7 \\
GUAIANO & 31.2 & 53.0 & 43.8 & - & 26.2 \\
BISABOLANO & 7.4 & 9.5 & 18.8 & - & 6.2 \\
EUDESMANO & 11.4 & - & - & - & 3.6 \\
EREMOFILANO & 1.8 & 8.9 & - & - & 2.7 \\
ESQ6 & - & 9.8 & - & - & 2.4 \\
CAROTANO & - & 9.5 & - & - & 2.3 \\
PRE-SEICHELANO & - & 9.2 & - & - & 2.2 \\
\hline
\end{tabular}

SUBESQUELETO: $10[11 \mathrm{OH}]-100.0 \%$ 
<smiles>CC1(C)CC2[C@@H](O)C[C@]3(C=O)C[C@]3(C)[C@@H]2C1</smiles>

Esqueleto MARASMANO
Phytochemistry, 1997, 45, 1569

Russula cuprea (Russulaceae)

RMN ${ }^{13} \mathbf{C}\left(\mathbf{C D C l}_{3}, \mathbf{C 1}\right.$ - C15) : 45,3 (t); 45,0 (d); 30,3 (s); 23,7 (t); 202,2 (d); 39,7 (s); 35,4 (d); 70,7 $(\mathrm{d}) ; 40,5$ (d); 43,3 (t); 37,1 (s); 20,3 (q); 63,5 (t); 31,0 (q), 31.,9 (q).

SUBESTRUTURAS PROPOSTAS PELO SISCONST:<smiles>CC1CC(C)(C)CC1C(C)(C)C</smiles><smiles>CC(C)C(C)CC(C)(C)C</smiles>

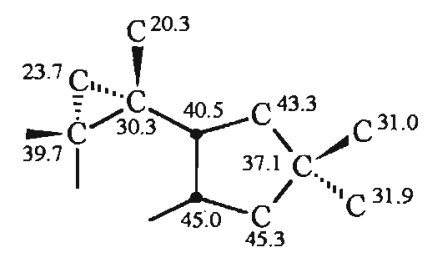

PROBABILIDADE DE ESQUELETO (\%)

\begin{tabular}{lccccc}
\hline ESQUELETO & SISCONST & C13MACH & SISOCBOT & REGRAS & GLOBAL \\
\hline \hline MARASMANO & 23.7 & 20.8 & - & 50.0 & 30.6 \\
ISOLACTARANO & - & - & - & 50.0 & 18.0 \\
PROTOLLUDANO & 22.0 & 30.3 & - & - & 14.3 \\
THUJOPSANO & 15.8 & - & - & - & 5.1 \\
EREMOFLANO & - & 19.5 & - & - & 4.7 \\
LONGIPINANO & 12.4 & - & - & - & 4.0 \\
4-14-CARIOFILANO & 10.2 & - & - & - & 3.3 \\
ARISTOLANO & 2.3 & 9.9 & - & - & 3.1 \\
\hline
\end{tabular}

SUBESQUELETO: 42[sem substituintes]. 


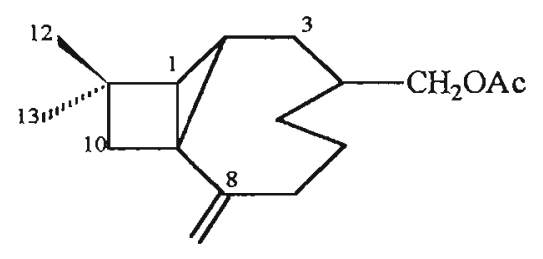

Phytochemistry, 1997, 45, 343

Euphorbia wangii (Euphorbiaceae)

Esqueleto 2-9-CARIOFILANO

RMN ${ }^{13} \mathrm{C}\left(\left(\mathrm{CD}_{3}\right)_{2} \mathrm{CO}, \mathrm{C1}-\mathrm{C15}\right): 61,7$ (d); 58,0 (d); 26,7 (t); 48,1 (d); 32,9 (t); 33,6 (t); 30,0 (t); 151,8 (s); 54,3 (s); 46,0 (t); 42,4 (s); 26,9 (q); 26,2 (q); 108,5 (t); 65,3 (t).

SUBESTRUTURA PROPOSTA PELO SISCONST:

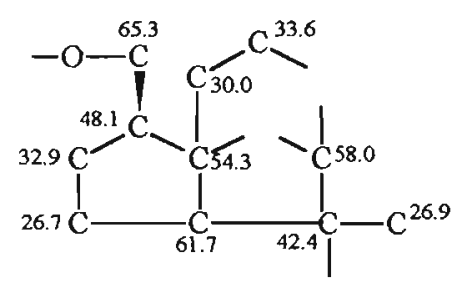

PROBABILIDADE DE ESQULETO (\%)

\begin{tabular}{lccccc}
\hline ESQUELETO & SISCONST & CI3MACH & SISOCBOT & REGRAS & GLOBAL \\
\hline \hline PROTOILUDANO & - & - & - & 91.0 & 32.8 \\
CEDRANO & 33.2 & 21.8 & - & - & 15.9 \\
VETISPIRANO & 13.4 & 19.2 & - & - & 8.9 \\
CARIOFILANO & 6.4 & - & 50.0 & - & 6.0 \\
TRIFARANO & 10.7 & 9.5 & - & - & 5.7 \\
GUAIANO & - & 19.9 & -- & - & 4.8 \\
EUDESMANO & 4.8 & 10.0 & - & - & 3.9 \\
CADINANO & - & 9.8 & - & - & 2.4 \\
\hline
\end{tabular}

SUBESQUELETO: $117[1 \mathrm{OR}]$ - $91.0 \%$ 


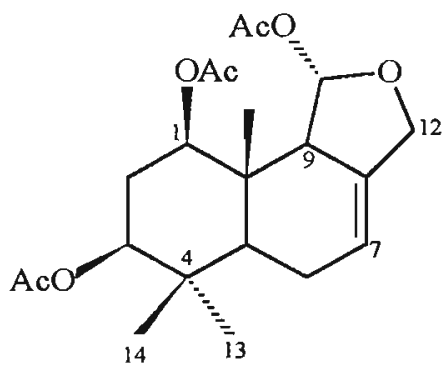

Esqueleto Drimano

Phytochemistry, 1996, 41, 1119 Nephrolepis biserrata (Davalliaceae)

$\mathbf{R M N}{ }^{13} \mathbf{C}\left(\mathrm{CDCl}_{3}, \mathbf{C 1}\right.$ - C15) : 77,5 (d); 29,3 (t); 76,2 (d); 37,2 (s); 46,7 (d); 22,5 (d); 117,0 (d); 134,9 (s); 57,7 (d); 37,4 (s); 98,2 (d); 69,4 (t); 27,1 (q); 15,5 (q); 9,1 (q).

SUBESTRUTURAS PROPOSTAS PELO SISCONST :<smiles>CO[C@H]1CC=C(C)[C@H](C)[C@H]1C</smiles>

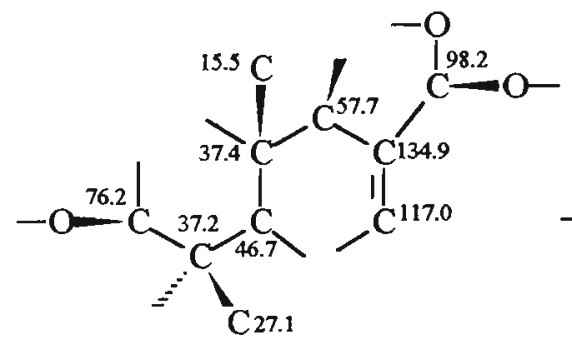<smiles>COC(OC)[C@@H]1C=CC(C(C)(C)C(C)OC)C(C)(C)[C@@H]1C</smiles>

PROBABILIDADE DE ESQUELETO (\%)

\begin{tabular}{lccccc}
\hline ESQUELETO & SISCONST & C13MACH & SISOCBOT & REGRAS & GLOBAL \\
\hline \hline DRIMANO & 86.7 & 49.9 & - & 100.0 & 75.7 \\
EUDESMANO & 8.1 & 21.0 & - & - & 7.6 \\
EREMOFILANO & - & 19.3 & - & - & 4.6 \\
CARIOFILANO & - & - & 50.0 & - & 4.0 \\
FARNESANO & - & 9.8 & - & - & 2.4 \\
OUTROS & 4.0 & - & - & - & 1.3 \\
14-15-CARIOFILANO & - & - & 16.7 & - & 1.3 \\
MONOCICLOFARNESANO & 1.2 & - & - & - & 0.4 \\
\hline
\end{tabular}

SUBESQUELETOS: 13 [7EN] - 94.0\%; 13[3OR] - 100.0\%. 


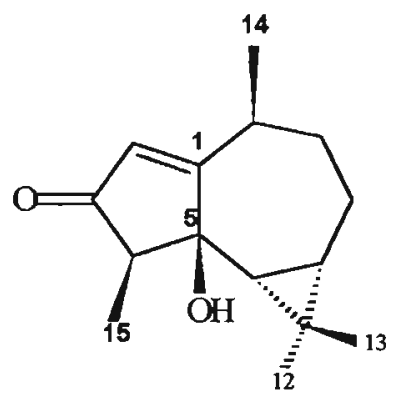

Phytochemistry, 1996, 41, 575

Esqueleto AROMADENDRANO Heteroscyphus coalitus (Jungermanniceae)

RMN ${ }^{13} \mathrm{C}\left(\mathrm{CDCl}_{3}, \mathrm{C1}\right.$ - C15) : 184,4 (s); 132,4 (s); 209,3 (s); 53,8 (d); 83,1 (s); 36,5 (d); 27,7 (d); 22,3 (t); 32,2 (t); 40,1 (d); 18,1 (s); 15,1 (q); 30,2 (q);25,6 (q);11,1 (q).

SUBESTRUTURAS PROPOSTAS PELO SISCONST:
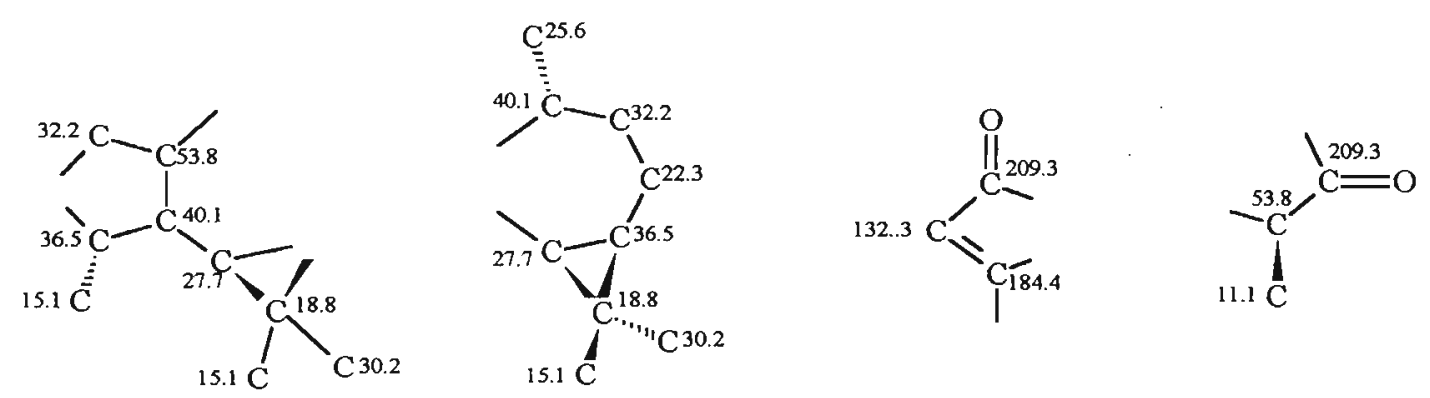

PROBABILIDADE DE ESQUELETO (\%)

\begin{tabular}{lccccc}
\hline ESQUELETO & SISCONST & C13MACH & SISOCBOT & REGRAS & GLOBAL \\
\hline \hline AROMADENDRANO & 86.8 & 39.1 & - & 100.0 & 73.2 \\
CADINANO & 7.1 & 10.5 & 9.4 & - & 5.5 \\
EUDESMANO & 2.0 & 2.0 & 25.0 & - & 3.1 \\
$11,12,14-T R I N O R-O X E P I N A$ & - & 10.9 & - & - & 2.6 \\
ARISTOLANO & - & 10.0 & - & - & 2.4 \\
ESQ5 & - & 9.9 & - & - & 2.4 \\
ESQ35 & - & 9.8 & - & - & 2.3 \\
OPLOPANANO & 2.5 & - & - & - & 0.8 \\
\hline
\end{tabular}

SUBESQUELETO: 87 [sem substituintes]. 


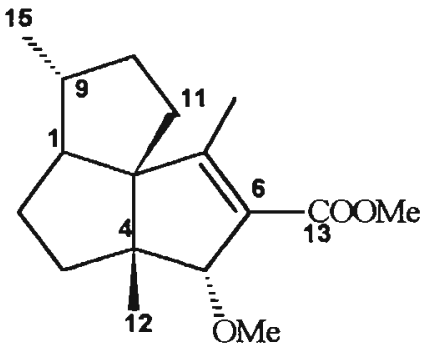

Phytochemistry, 1996, 41, 837

Esqueleto SILFIPERFOLANO Artemisia chamaemelifolia (Compositae)

$\mathbf{R M N}{ }^{13} \mathrm{C}\left(\mathrm{CDCl}_{3}, \mathbf{C} 1\right.$ - C15) : 58,6 (d); 30,1 (t); 35,4 (t); 52,5 (s); 94,5 (d); 126,0 (s); 163,6 (s); 73,2 (s); 41,4 (d); 36,9 (t); 29,5 (t); 25,2 (q); 166,4 (s); 13,5 (q); 19,3 (q); 5-OMe - 60,6 (s); 13-OMe - 50,9(s).

SUBESTRUTURAS PROPOSTAS PELO SISCONST:
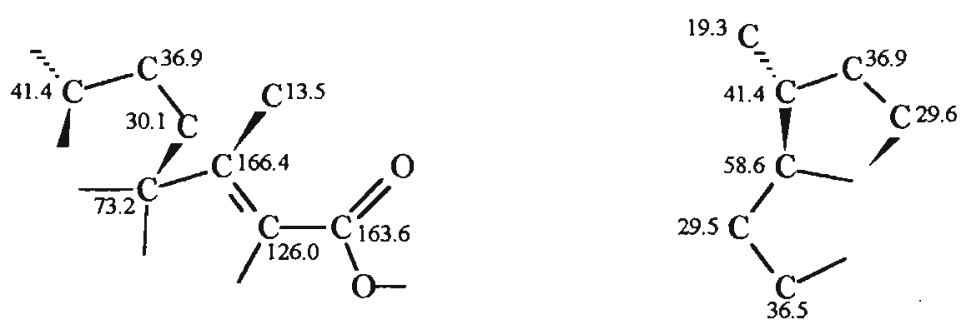

PROBABILIDADE DE ESQUELETO (\%)

\begin{tabular}{lccccc}
\hline ESQUELETO & SISCONST & C13MACH & SISOCBOT & REGRAS & GLOBAL \\
\hline \hline SILFIPERFOLANO & 29.4 & 32.0 & 12.5 & 100.0 & 54.1 \\
CEDRANO & 23.5 & - & - & - & 7.5 \\
GUAIANO & 20.0 & - & - & - & 6.4 \\
EUDESMANO & 4.7 & 9.7 & 25.0 & - & 5.8 \\
VETISPIRANO & 11.8 & - & - & - & 3.8 \\
CADINANO & -- & 9.7 & 9.4 & - & 3.1 \\
13-NOR-SILFIPERFOLANO & 1.2 & 9.3 & 3.1 & - & 2.9 \\
BISABOLANO & - & 9.6 & 6.3 & - & 2.8 \\
\hline
\end{tabular}

SUBESQUELETOS: 119 [6EN] - 100.0\% 


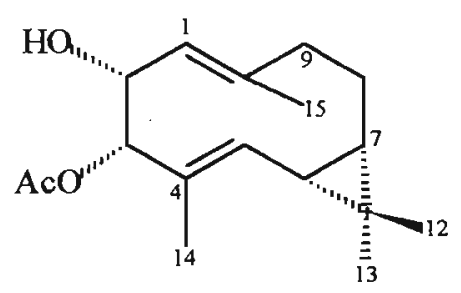

Phytochemistry, 1996, $\underline{43}, 83$

Esqueleto BICICLOGERMACRANO Heteroscyphus planus (Jungermanniaceae)

RMN ${ }^{13} \mathrm{C}\left(\mathrm{CDCl}_{3}, \mathbf{C 1}\right.$ - C15) : 122,9 (d); 70,4 (d); 82,1 (d); 126,6 (s); 128,4 (d); 27,1 (d); 31,2 (d); 27,1 (t); 37,6 (t); 143,3 (s); 21,0 (s); 29,0 (q);15,3 (q); 15,9 (q);21,9 (q); OAc - 170,7 (s); 21,2 (q).

SUBESTRUTURAS PROPOSTAS PELO SISCONST:
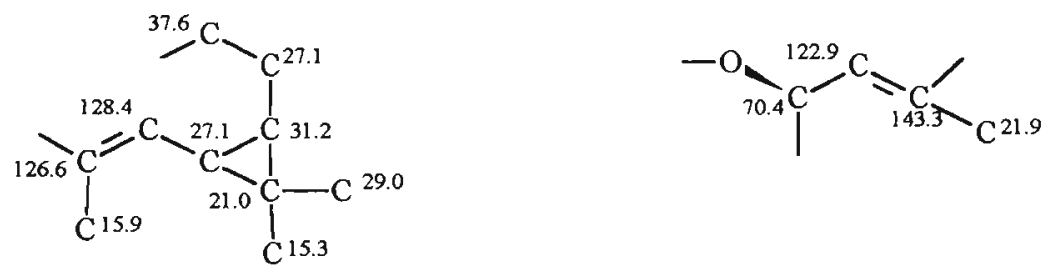

PROBABILIDADE DE ESQUELETO (\%)

\begin{tabular}{lccccc}
\hline ESQUELETO & SISCONST & C13MACH & SISOCBOT & REGRAS & GLOBAL \\
\hline \hline CADINANO & - & 40.1 & 100.0 & - & 17.6 \\
BICICLOGERMACRANO & 38.7 & 20.7 & - & 2.8 & 17.4 \\
BISABOLANO & 29.4 & 9.9 & - & - & 11.8 \\
AROMADENDRANO & 19.6 & 10.1 & - & - & 8.7 \\
14-NOR-CARIOFLANO & - & 20.0 & - & - & 4.8 \\
OPLOPANANO & - & 9.6 & - & - & 2.3 \\
FARNESANO & 6.7 & - & - & - & 2.1 \\
ESQ14 & 2.1 & - & - & 2.8 & 0.7 \\
\hline
\end{tabular}

MACRONO: Acetato - 170,7s; 21,2q (erro médio $=0.950$ ). 


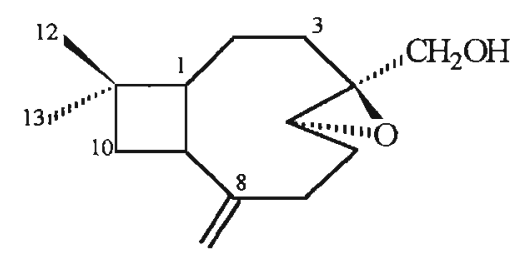

Phytochemistry, 1997, 4 5, 343

Esqueleto CARIOFILANO

Euphorbia wargii (Euphorbiaceae)

RMN ${ }^{13}$ C ( acetona-d 6 , C1-C15): 50,6 (d); 27,1 (t); 35,9 (t); 62,6 (s); 63,6 (d); 30,7 (t); 29,7 (t); 152,6 $(\mathrm{s}) ; 49,2$ (d); 40,1 (t); 34,6 (s); 29,9 (q); 21,8 (q); 113,0 (t); 62,0 (t).

SUBESTRUTURAS PROPOSTAS PELO SISCONST:
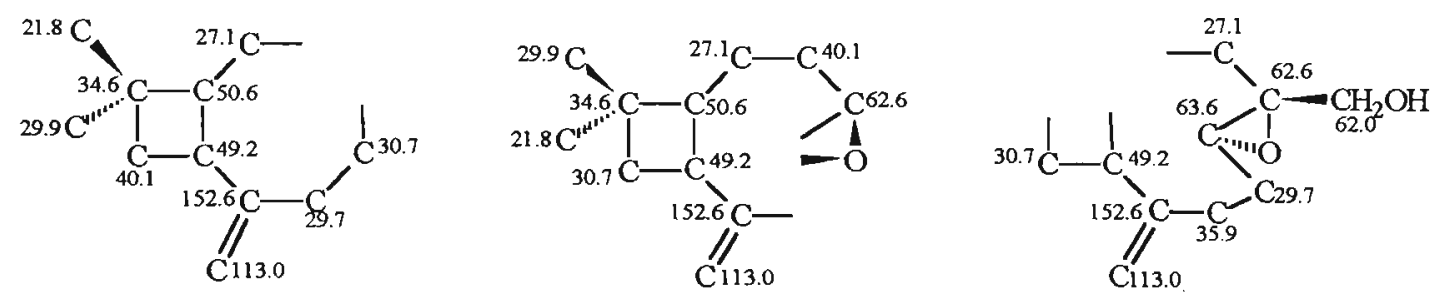

PROBABILIDADE DE ESQUELETO (\%)

\begin{tabular}{lccccc}
\hline ESQUELETO & SISCONST & C13MACH & SISOCBOT & REGRAS & GLOBAL \\
\hline \hline CARIOFILANO & 56.5 & 63.6 & 50.0 & 100.0 & 73.3 \\
EUDESMANO & 12.5 & 8.8 & - & - & 6.1 \\
GERMACRANO & - & 18.5 & - & - & 4.4 \\
GUAIANO & 2.9 & 9.1 & - & - & 3.1 \\
MONOCICLOFARNESANO & 7.2 & - & - & - & 2.3 \\
14NOR-CARIOFILANO & 4.5 & - & - & - & 1.4 \\
12NOR-CARIOFLLANO & 4.5 & - & - & - & 1.4 \\
OUTROS & 3.8 & - & - & - & 1.2 \\
\hline
\end{tabular}

SUBESQUELETOS: $122[8(14) E N]-92.6 \% ; 122[4,5 E P]-100.0 \%$ 


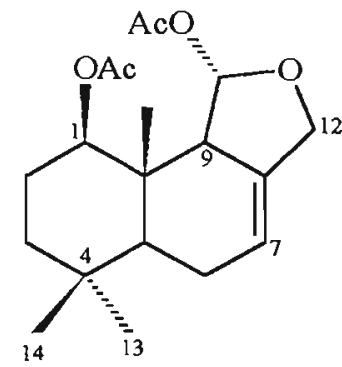

Phytochemistry, 1996, $\underline{41}, 1119$

Esqueleto DRIMANO Nephrolepis biserrata (Davalliaceae)

RMN ${ }^{13} \mathrm{C}\left(\mathrm{CDCl}_{3}, \mathbf{C 1}-\mathrm{C15}\right): 81,4$ (d); 24,2 (t); 39,6 (t); 32,7 (s); 48,9 (d); 23,3 (t); 117,6 (d); 135,1 (s); 57,9 (d); 37,4 (s); 98,8 (d); 69,6 (t); 32,3 (q); 21,3 (q); 9,1 (q); OAc - 21,3 (q); 21,3 (q); 170,5 (s); 171,1 (s).

SUBESTRUTURAS PROPOSTAS PELO SISCONST:<smiles>CC[C@H]1C[C@H](OC)[C@@H](CC)C1(C)C</smiles><smiles>CCC(=O)C(C)(C)[C@@H](CC)C(C)(C)C(C)C</smiles><smiles>CCC(C(C)(C)C)C(C)(C)CC</smiles><smiles>CC(CC=C1CO[C@H](O)[C@@H]1C)C(C)(C)C</smiles>

PROBABLIDADE DE ESQUELETO (\%)

\begin{tabular}{lccccc}
\hline ESQUELETO & SISCONST & C13MACH & SISOCBOT & REGRAS & GLOBAL \\
\hline \hline DRIMANO & 81.8 & 61.6 & - & 100.0 & 77.0 \\
EUDESMANO & 11.6 & 29.1 & - & - & 10.7 \\
IPIONANO & - & 9.3 & - & - & 2.2 \\
CARIOFILANO & 3.5 & - & - & - & 1.1 \\
OUTROS & 2.0 & - & - & - & 0.6 \\
\hline
\end{tabular}

SUBESQUELETOS: 13[7EN] - 94\%; outros drimanos - 6\% 
<smiles>Cc1coc2c1[C@@H](O)[C@]1(C)C(=CCC[C@H]1C)C2</smiles>

Esqueleto EREMOFILANO
Phytochemistry, 1998, 47, 57

Senecio auricula (Compositae)

RMN ${ }^{13} \mathbf{C}\left(\mathbf{C D C l}_{3}, \mathbf{C 1}\right.$ - C15) : 124,3 (d); 21,3 (t); 31,3 (t); 31,9 (d); 42,5 (s); 72,1 (d); 119,7 (s); 151,3

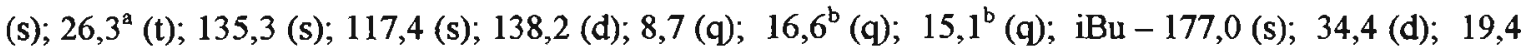
(q); 18,7 (q).

SUBESTRUTURAS PROPOSTAS PELO SISCONST:

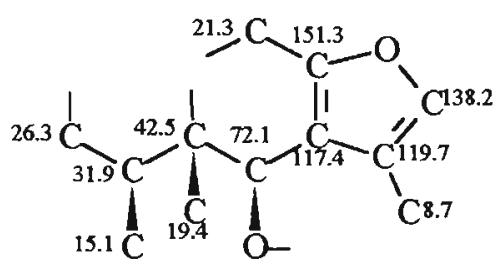<smiles>CCC1=C(CC)C(C)(C)C(C)(C)C1O</smiles>

PROBABILIDADE DE ESQUELETO (\%)

\begin{tabular}{lccccc}
\hline ESQUELETO & SISCONST & C13MACH & SISOCBOT & REGRAS & GLOBAL \\
\hline \hline EREMOFILANO & 73.3 & 72.2 & 46.4 & 91.0 & 77.3 \\
CADINANO & - & 18.6 & - & - & 4.5 \\
EUDESMANO & - & - & - & 9.0 & 3.2 \\
ARISTOLANO & - & 9.3 & - & - & 2.2 \\
FURODISANO & 4.8 & - & - & - & 1.5 \\
GERMACRANO & 3.3 & - & 3.6 & - & 1.3 \\
BISABOLANO & 2.2 & - & 7.1 & - & 1.3 \\
PINGUISANO & 3.3 & - & - & - & 1.1 \\
\hline
\end{tabular}

SUBESQUELETO: 6 [ 8,12FURANO] - 91.0\% 


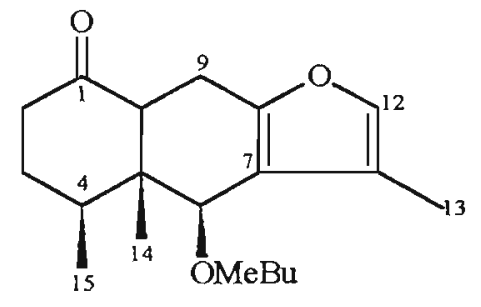

Esqueleto EREMOFILANO
Phytochemistry, 1998, 47, 57

Senecio auricula (Compositae)

$\mathbf{R M N}{ }^{13} \mathrm{C}\left(\mathrm{CDCl}_{3}, \mathrm{C1}-\mathrm{C15}\right): 209,0(\mathrm{~s}) ; 36,0(\mathrm{t}) ; 29,8(\mathrm{t}) ; 30,3$ (d); 44,1 (s); 67,9 (d); 116,0 (s); 149,8 (s); 22,3 (t); 51,1 (d); 119,4 (s); 138,4 (d); 8,2 (q); 17, $8^{\mathrm{a}}$ (q); 14,8 (q); MeBu - 176,6 (s); 41,2 (d); 26,4 $(\mathrm{t}) ; 17,0^{\mathrm{a}}$ (q); $11,7(\mathrm{q})$.

SUBESTRUTURAS PROPOSTAS PELO SISCONST:

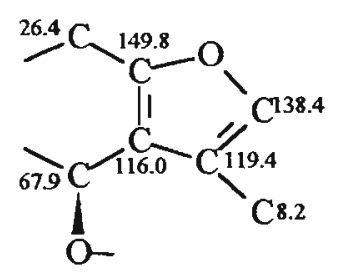<smiles>CCC(CC)[C@@H](C)C(C)=O</smiles>

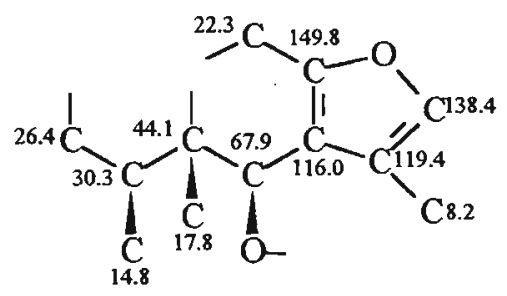

PROBABILIDADE DE ESQUELETO (\%)

\begin{tabular}{lccccc}
\hline ESQUELETO & SISCONST & C13MACH & SISOCBOT & REGRAS & GLOBAL \\
\hline \hline EREMOFILANO & 36.7 & 70.7 & 46.4 & 91.0 & 65.2 \\
1-3-EUDESMANO & 27.8 & 9.4 & - & - & 11.2 \\
EUDESMANO & 8.2 & 10.1 & - & 9.0 & 8.3 \\
ELEMANO & 3.7 & 9.8 & - & - & 3.5 \\
CADINANO & 6.5 & - & - & - & 2.1 \\
OPLOPANANO & - & - & 25.0 & - & 2.0 \\
VETTSPIRANO & 5.7 & - & - & - & 1.8 \\
OUTROS & 3.7 & - & - & - & 1.2 \\
\hline
\end{tabular}

SUBESQUELETO: 6 [ 8,12FURANO ] - 91.0\% 
<smiles></smiles>

Esqueleto CADINANO
Phytochemistry, 1997, 45, 1523

Thespesia populnea (Malvaceae)

$\mathbf{R M N}{ }^{13} \mathrm{C}\left(\mathrm{CDCl}_{3}, \mathbf{C 1}-\mathrm{C15}\right): 115,1$ (s) ; 143,6 (s); 181,3 (s); 115,0 (s); 157, 3 (s); 131,0 (s); 139,4 (s); 197,1 (s); 44,5 (t); 27,5 (d); 26,4 (d); 71,9 (t); 16,2 (q); 20,6 (q); 8,0 (q);

SUBESTRUTURAS PROPOSTAS PELO SISCONST:<smiles>C=C(C)C(C)=C1CC(C#N)COC1=C(C)C(C)=O</smiles><smiles>CC(=O)[C@@H](O)[C@H](C)[C@H](C)[C@@H](C)C(C)=O</smiles>

PROBABILIDADE DE ESQUELETO (\%)

\begin{tabular}{lccccc}
\hline ESQUELETO & SISCONST & C13MACH & SISOCBOT & REGRAS & GLOBAL \\
\hline \hline CADINANO & 91.7 & 9.6 & 75.0 & 100.0 & 73.6 \\
AROMADENDRANO & - & 30.4 & 8.3 & - & 8.0 \\
BICICLOGERMACRANO & - & 19.6 & - & - & 4.7 \\
14(->6)-EREMOFILANO & 8.3 & - & - & - & 2.7 \\
EREMOFLLANO & - & 10.9 & - & - & 2.6 \\
GERMACRANO & - & 10.1 & -- & - & 2.4 \\
11-NOR-PTEROSIN & - & 9.7 & - & - & 2.3 \\
4,5-SECO-EREMOFLANO & - & 9.7 & - & - & 2.3 \\
\hline
\end{tabular}

SUBESQUELETO: $10[2 \mathrm{OXO} ; 1(10), 6,8 \mathrm{EN}]-100.0 \%$ 


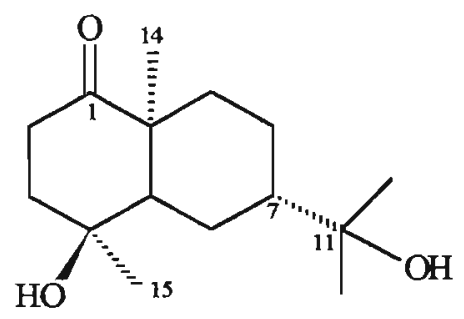

Esqueleto EUDESMANO

Phytochemistry, 1997, 44, 459 Laggera pteredonta (Compositae)

RMN ${ }^{13} \mathrm{C}\left(\left(\mathrm{CD}_{3}\right)_{2} \mathbf{C O}, \mathbf{C 1} 1 \mathrm{C15}\right): 215,8(\mathrm{~s}) ; 33,6(\mathrm{t}) ; 36,0(\mathrm{t}) ; 70,3(\mathrm{~s}) ; 48,0(\mathrm{~d}) ; 21,6(\mathrm{t}) ; 42,4$ (d); 22,1 (t); 41,2 (t); 46,5 (s); 73,4 (s); 29,5 (q); 30,1 (q); 19,2 (q); 23,7 (q).

SUBESTRUTURAS PROPOSTAS PELO SISCONST:
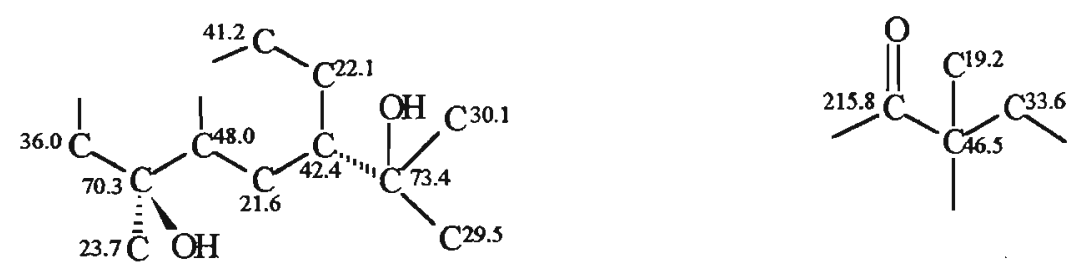

PROBABILIDADE DE ESQUELETO (\%)

\begin{tabular}{lccccc}
\hline \multicolumn{1}{c}{ ESQUELETO } & SISCONST & C13MACH & SISOCBOT & REGRAS & GLOBAL \\
\hline \hline EUDESMANO & 34.5 & 29.7 & 100.0 & 100.0 & 62.5 \\
BISABOLANO & 5.7 & 20.5 & - & - & 6.7 \\
CADINANO & 4.5 & 20.1 & - & - & 6.3 \\
ESQ31 & 18.2 & - & - & - & 5.8 \\
OUTROS & 12.5 & - & - & - & 4.0 \\
LONGICICLANO & - & 10.2 & - & - & 2.4 \\
ESTERPURANO & - & 9.8 & - & - & 2.3 \\
CARIOFLLANO & - & 9.6 & - & - & 2.3 \\
\hline
\end{tabular}

SUBESQUELETOS: $1[4,11 \mathrm{OR}]-100.0 \% ; 1[11 \mathrm{OR}]-90.6 \%$ 


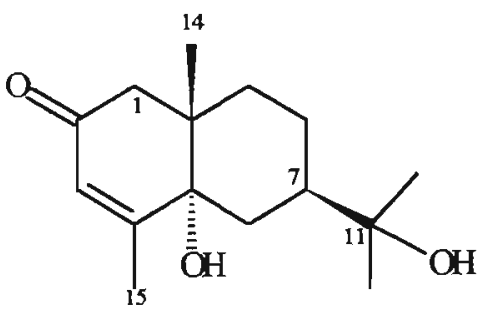

Esqueleto EUDESMANO
Phytochemistry, 1997, $\underline{44}, 459$

Laggera pteredonta (Compositae)

RMN ${ }^{13} \mathrm{C}$ ( ( CD $\left._{3}\right)_{2}$ CO, C1-C15) : 47,7 (t); 198,4 (s); 125,5 (d); 168,2 (s); 76,2 (s); 35,4 (t); 42,9 (d); $23,0(\mathrm{t}) ; 76,2$ (t); 41,5 (s); 71,8 (s); 23,2 (q); 27,1 (q); 18,2 (q); 28,1 (q).

SUBESTRUTURAS PROPOSTAS PELO SISCONST:
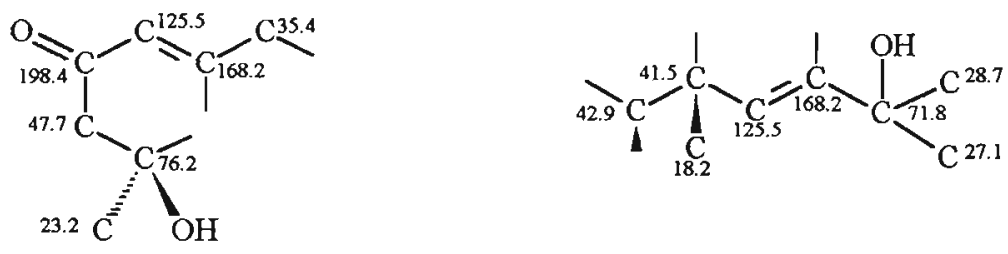

PROBABILIDADE DE ESQUELETO (\%)

\begin{tabular}{lccccc}
\hline ESQUELETO & SISCONST & C13MACH & SISOCBOT & REGRAS & GLOBAL \\
\hline \hline EUDESMANO & 15.6 & 29.0 & 100.0 & 90.6 & 52.5 \\
EREMOFILANO & 28.7 & - & - & - & 9.2 \\
BISABOL_ANO & 10.7 & 9.8 & - & - & 5.7 \\
CHAMIGRANO & 9.8 & 10.7 & - & - & 5.7 \\
CADINANO & - & 19.9 & - & - & 4.8 \\
ESQ31 & 13.9 & - & - & - & 4.4 \\
HIMACHALANO & - & 10.2 & - & - & 2.4 \\
ESQ6 & - & 9.9 & - & - & 2.4 \\
\hline
\end{tabular}

SUBESQUELETO : $1 .[11 \mathrm{lOR}]-90.6 \%$ 


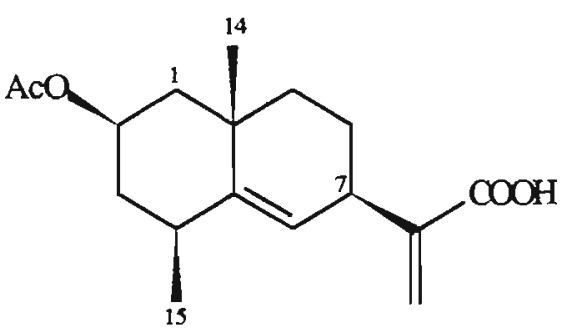

Phytochemistry, 1997, $\underline{44}, 459$

Esqueleto EUDESMANO Laggera pteredonta (Compositae)

RMN ${ }^{13}$ C (acetona-d 6 , C1-C15) : 47,2 (t); 67,8 (d); 39,0 (t); 39,4 (d); 147,8 (s); 126,0 (d); 42,0 (d); 27,5 (t); 42,0 (t); 39,4 (s); 146,7 (s); 168,5 (s); 123,1 (t); 28,2 (q); 19,3 (q); OAc - 170,4 (s); 21,2 (q).

SUBESTRUTURAS PROPOSTAS PELO SISCONST:<smiles>C=C(C(=O)OC)[C@@H](C)[CH][C@H](C)C(C)(C)CC</smiles><smiles>C=C(C)C(CC)C(C)(C)C(C)C(C)COC</smiles>

PROBABILIDADE DE ESQUELETO (\%)

\begin{tabular}{lccccc}
\hline ESQUELETO & SISCONST & C13MACH & SISOCBOT & REGRAS & GLOBAL \\
\hline \hline EUDESMANO & 26.7 & 20.9 & 100.0 & 94.0 & 55.4 \\
EREMOFLANO & 37.0 & 9.6 & - & - & 14.1 \\
LONGIPINANO & 5.2 & 19.9 & - & - & 6.4 \\
13-NOR-ELEMANO & 3.7 & 10.1 & - & - & 3.6 \\
GUAIANO & 2.2 & 9.9 & - & - & 3.1 \\
BISABOLANO & - & 10.3 & - & - & 2.5 \\
CARIOFILANO & - & 9.7 & - & - & 2.3 \\
CAROTANO & - & 9.7 & - & - & 2.3 \\
\hline
\end{tabular}

SUBESQUELETO: 1 [ 13OXO; 13OR; 11EN] - 94.0\%

MACRONO : Acetato - 168,5s; 21,2q (erro médio $=0.350$ ). 
<smiles>C=C(C(=O)O)[C@@H]1CC[C@]2(C)C[C@H](OC(C)=O)CC(=S)C2C1</smiles>

Phytochemistry, 1997, 44, 459

Esqueleto EUDESMANO Laggera pteredonta (Compositae)

RMN ${ }^{13}$ C (acetona-d ${ }_{6}$, C1-C15) : 47,3 (t); 70,8(d); 42,8 (t); 146,5 (s); 49,8 (d); 26,6 (t); 40,4 (d); 30,0 $(\mathrm{t}) ; 41,2(\mathrm{t}) ; 35,9(\mathrm{~s}) ; 146,6(\mathrm{~s}) ; 168,3(\mathrm{~s}) ; 123,7(\mathrm{t}) ; 24,0(\mathrm{q}) ; 109,1(\mathrm{t}) ;$ OAc - 170,5 (s); $21,2(\mathrm{q})$.

SUBESTRUTURAS PROPOSTAS PELO SISCONST:
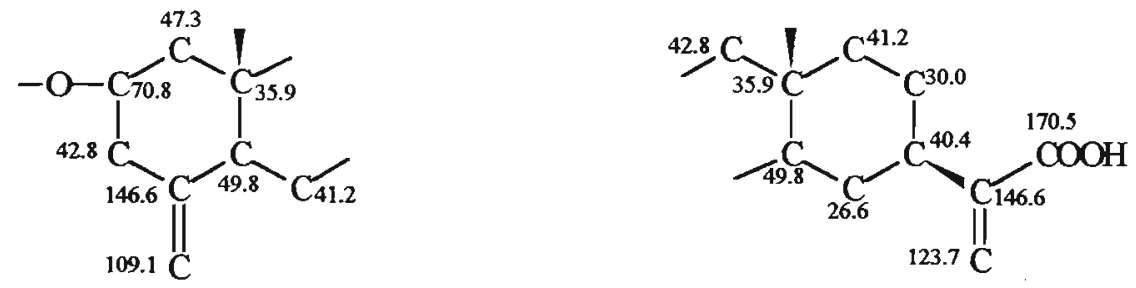

PROBABILDADE DE ESQUELETO (\%)

\begin{tabular}{lccccc}
\hline ESQUELETO & SISCONST & CI3MACH & SISOCBOT & REGRAS & GLOBAL \\
\hline \hline EUDESMANO & 42.5 & 80.7 & 100.0 & 94.0 & 74.8 \\
EREMOFILANO & 14.6 & - & - & - & 4.7 \\
CARIOFILANO & 11.4 & - & - & - & 3.6 \\
OUTROS & 10.2 & - & - & - & 3.3 \\
4,5-SECO-CARIOFILANO & 10.0 & - & - & - & 3.2 \\
2-10-HIMACHALANO & - & 9.7 & - & - & 2.3 \\
12,13-DINOR-ELEMANO & - & 9.6 & - & - & 2.3 \\
13-NOR-ELEMANO & 4.8 & - & - & - & 1.5 \\
\hline
\end{tabular}

SUBESQUELETO: 1 [ 13OXO; 13OR; 11EN] - 94.0\%

MACRONO: Acetato - 168,3s; 21,2q (erro médio $=0.450$ ). 
<smiles>CC(C)=C1CC[C@@]2(C)C([C@H]1O)[C@](O)(F)CC[C@@H]2O</smiles>

Phytochemistry, 1997, 44, 459

RMN ${ }^{13} \mathbf{C}$ (piridina-d 6 , C1-C15) : 79,3 (d); 29,4 (t); 36,7 (t); 72,7 (s); 47,9 (d); 73,1 (d); 51,2 (d); 23,4 $(\mathrm{t}) ; 41,4$ (t); 41,5 (s); 25,9 (d); 24,5 (q); 25,1 (q); 14,7 (q); 22,6 (q).

SUBESTRUTURAS PROPOSTAS PELO SISCONST:<smiles>C[C@@H]1[C@H](O)[C@H](C)[C@](C)(O)C1(C)C</smiles><smiles>CC(C)C(C)C(C)O</smiles>

PROBABILIDADE DE ESQUELETO (\%)

\begin{tabular}{lccccc}
\hline ESQUELETO & SISCONST & C13MACH & SISOCBOT & REGRAS & GLOBAL \\
\hline \hline EUDESMANO & 68.2 & 50.2 & 100.0 & 90.6 & 74.5 \\
BULATANO & - & 9.7 & - & 9.4 & 5.7 \\
ELEMANO & 3.3 & 9.6 & - & - & 3.4 \\
CADINANO & 2.9 & 9.7 & - & - & 3.3 \\
15-NOR-BOURBONANO & - & 11.2 & - & - & 2.7 \\
OUTROS & 8.9 & - & - & - & 2.8 \\
BISABOLANO & - & 9.7 & - & - & 2.3 \\
CAROTANO & 1.8 & - & - & - & 0.6 \\
\hline
\end{tabular}

SUBESQUELETOS: $1[11 \mathrm{OR}]-90.6 \% ; 1[4,6 \mathrm{OR}]-78.9 \% ; 1[1,4 \mathrm{OR}]-58.3 \%$. 


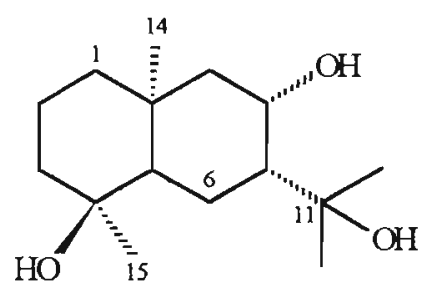

Phytochemistry, 1997, 44, 459

Esqueleto EUDESMANO Laggera pteredonta (Compositae)

RMN ${ }^{13} \mathrm{C}$ (acetona-d $\left.{ }_{6}, \mathrm{C1}-\mathrm{C15}\right): 43,5(\mathrm{t}) ; 21,0(\mathrm{t}) ; 44,3(\mathrm{t}) ; 71,6(\mathrm{~s}) ; 52,0$ (d); $21,5(\mathrm{t}) ; 48,4$ (d); 68,6 (d); 52,0 (t); 35,2 (s); 73,9 (s); 25,8 (q); 30,1 (q);23,3 (q); 23,8 (q).

SUBESTRUTURAS PROPOSTAS PELO SISCONST:
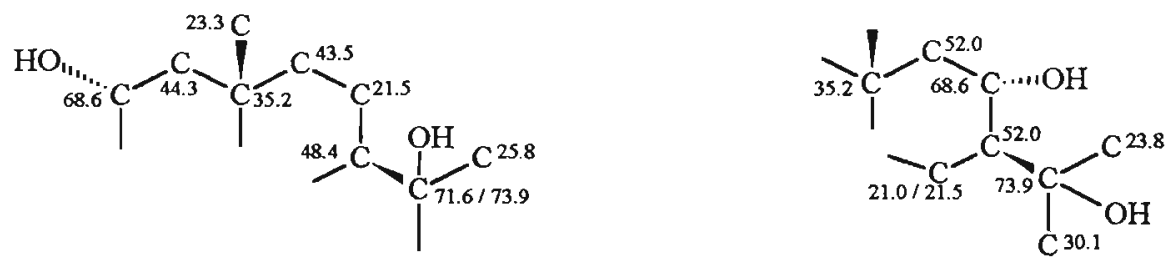

PROBABILIDADE DE ESQUELETO (\%)

\begin{tabular}{lccccc}
\hline \multicolumn{1}{c}{ ESQUELETO } & SISCONST & C13MACH & SISOCBOT & REGRAS & GLOBAL \\
\hline \hline EUDESMANO & 64.1 & 50.8 & 100.0 & 100.0 & 76.7 \\
LONGIPINANO & - & 20.5 & - & - & 4.9 \\
BISABOLANO & 4.9 & 9.3 & - & - & 3.8 \\
LACTARANO & 2.5 & 9.6 & - & - & 3.1 \\
CADINANO & 1.6 & 9.8 & - & - & 2.8 \\
ELEMANO & 4.9 & - & - & - & 1.6 \\
14-NOR-EUDESMANO & 4.7 & - & - & - & 1.5 \\
GUALANO & 4.5 & - & - & - & 1.4 \\
\hline
\end{tabular}

SUBESQUELETOS: $1[4,11 \mathrm{OR}]-100 \% ; 1[11 \mathrm{OR}]-90.6 \% ; 1[4 \mathrm{OR}]-55.8 \%$. 
<smiles>CC(=CCCC(C)C1(O)CC=C(C)CC1)CO</smiles>

Esqueleto BISABOLANO
Phytochemistry, 1997, $\underline{44}, 1519$

Santalum austrocaledonicum (Santalaceae)

RMN ${ }^{13} \mathbf{C}\left(\mathbf{C D C l}_{3}, \mathbf{C 1}-\mathbf{C 1 5}\right): 133,9(\mathrm{~s}) ; 118,4(\mathrm{~d}) ; 35,0(\mathrm{t}) ; 72,2(\mathrm{~s}) ; 30,4(\mathrm{t}) ; 27,0$ (t); 41,6 (d); 31,2 (t); $26,1(\mathrm{t}) ; 128,4(\mathrm{~d}) ; 134,6(\mathrm{~s}) ; 21,3(\mathrm{q}) ; 61,3(\mathrm{t}) ; 23,3$ (q); 13,7 (q).

\section{SUBESTRUTURAS PROPOSTAS PELO SISCONST:}
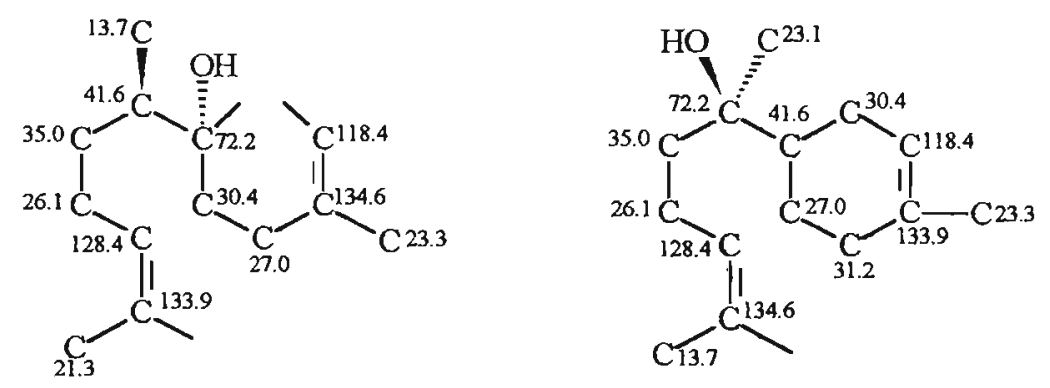

PROBABILIDADE DE ESQUELETO (\%)

\begin{tabular}{lccccc}
\hline ESQUELETO & SISCONST & C13MACH & SISOCBOT & REGRAS & GLOBAL \\
\hline \hline BISABOLANO & 57.9 & 19.7 & 100.0 & 50.0 & 49.3 \\
CADINANO & 18.1 & 51.3 & - & 50.0 & 36.1 \\
FARNESANO & 15.8 & - & - & - & 5.1 \\
ESQ51B & - & 9.7 & - & - & 2.3 \\
ACORANO & - & 9.6 & - & - & 2.3 \\
14-NOR-EREMOFLANO & - & 9.6 & - & - & 2.3 \\
OUTROS & 2.7 & - & - & - & 0.9 \\
GERMACRANO & 2.1 & - & - & - & 0.7 \\
\hline
\end{tabular}

SUBESQUELETOS : $129[7,110 X]-100.0 \% ; 10[1 \mathrm{OH}, 4 \mathrm{EN}]-100.0 \%$ 


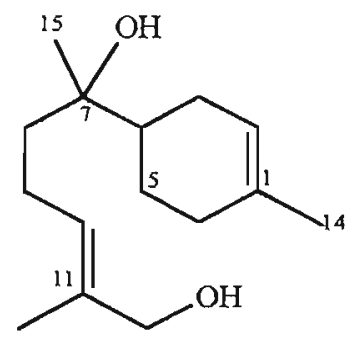

Phytochemistry, 1997, 44, 1519

Esqueleto BISABOLANO Santalum austrocaledonicum (Santalaceae)

$\mathbf{R M N}{ }^{13} \mathbf{C}\left(\mathrm{CDCl}_{3}, \mathbf{C 1 - C 1 5 ) :} 133,9(\mathrm{~s}) ; 120,7\right.$ (d); 26,1 (t); 43,7 (d); 24,1 (t); 31,1 (t); 74,6 (s); 39,4 (t); $21,9(\mathrm{t}) ; 128,7$ (d); 134,5 (s); 21,6 (q); 61,5 (t);23,5 (q); 23,9 (q).

SUBESTRUTURAS PROPOSTAS PELO SISCONST:
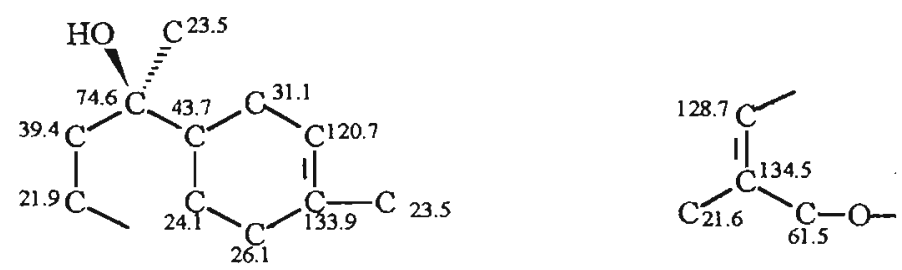

PROBABLIDADE DE ESQUELETO (\%)

\begin{tabular}{lccccc}
\hline ESQUELETO & SISCONST & C13MACH & SISOCBOT & REGRAS & GLOBAL \\
\hline \hline BISABOLANO & 63.1 & 80.5 & 100.0 & 100.0 & 83.5 \\
CADINANO & 11.5 & 19.5 & - & - & 8.4 \\
FARNESANO & 12.7 & - & - & - & 4.1 \\
15 $\rightarrow$ 1)-EUDESMANO & 3.7 & - & - & - & 1.2 \\
EUDESMANO & 3.0 & - & - & - & 1.0 \\
ESQ18 & 1.9 & - & - & - & 0.6 \\
GERMACRANO & 1.7 & - & - & - & 0.5 \\
OUTROS & 1.3 & - & - & - & 0.4 \\
\hline
\end{tabular}

SUBESQUELETO : $129[7,110 X]-100.0 \%$ 


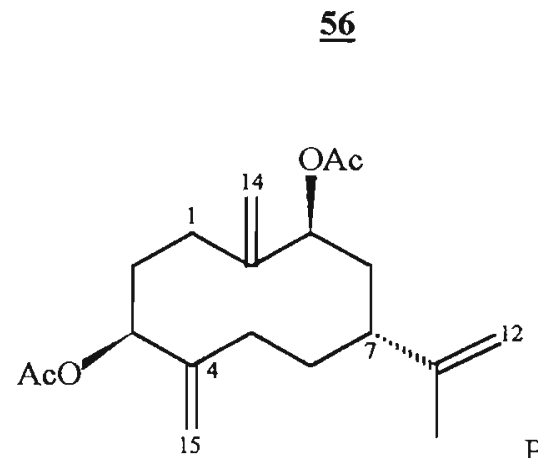

Phytochemistry, 1997, $\underline{44}, 1287$

Chrysanthemum morifolium (Compositae)

Esqueleto GERMACRANO

RMN ${ }^{13} \mathbf{C}\left(\mathbf{C D C l}_{3}, \mathbf{C 1}-\mathbf{C 1 5}\right): 24,8(\mathrm{t}) ; 29,7(\mathrm{t}) ; 76,0$ (d); 145,2 (s); 30,4 (t); 31,2 (t); 40,6 (d); 34,4 (t); 78,4 (d); 145,3 (s); 148,1 (s); 110,5 (t); 19,4 (q); 116,9 (t); 116,6 (t); OAc - 170,2 (s); 169,6 (s); 21,3 (q); $21,3(\mathfrak{q})$.

SUBESTRUTURAS PROPOSTAS PELO SISCONST:<smiles>CC(C)C(C)C(C)C(C)O</smiles><smiles>C=C(CC(C)C(C)(C)C)[C@@H](O)C(C)(C)C</smiles><smiles>C=C(C)C(CC)CCC</smiles>

PROBABLIDADE DE ESQUELETO (\%)

\begin{tabular}{lccccc}
\hline ESQUELETO & SISCONST & C13MACH & SISOCBOT & REGRAS & GLOBAL \\
\hline \hline GERMACRANO & 52.5 & 10.1 & - & 100.0 & 55.2 \\
GUAIANO & 22.5 & - & - & - & 7.2 \\
CADINANO & 3.3 & 19.7 & - & - & 5.8 \\
BISABOLANO & - & 12.7 & 25.0 & - & 5.0 \\
ESQ26 & 10.8 & - & - & - & 3.5 \\
COPAANO & - & 9.6 & - & - & 2.3 \\
TREMULANO & - & 9.6 & - & - & 2.3 \\
DRIMANO & - & 9.6 & - & - & 2.3 \\
\hline
\end{tabular}

SUBESQUELETO: $105[4(15), 10(14) \mathrm{EN}]-100.0 \%$

MACRONO: Acetatos - 170,2 s; 169,6 s; 21,3 q; 21,3 q. 


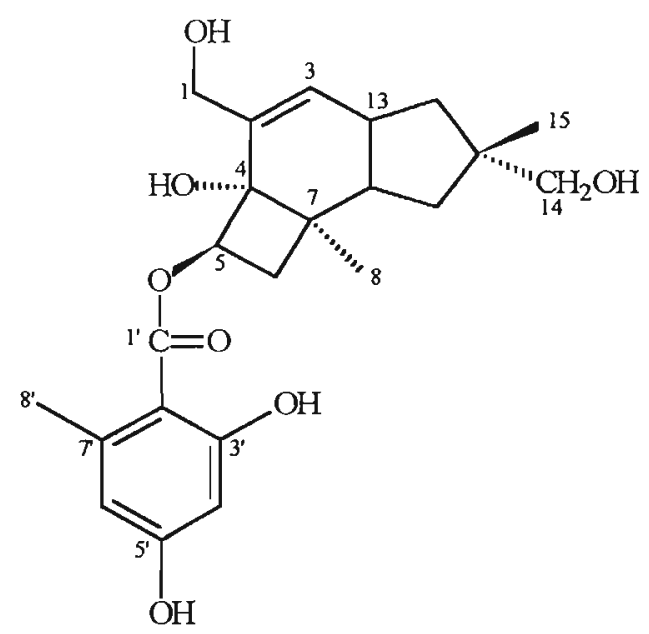

Phytochemistry, 1997, 44, 1473

Esqueleto PROTOLUDANO

Armillaria tabescens (Fungo)

RMN ${ }^{13} \mathrm{C}$ (acetona-d 6 , C1-C15): 64,5 (t); 135,6 (t); 132,6 (t); 77,7 (s); 77,0 (d); 33,7 (t); 39,7 (s); 22,1 (q); 44,8 (d); 37,6 (t); 44,5 (s); 43,5 (t); 39,8 (d); 72,4 (t); 27,1 (q); Orselinato - 172,0 (s); 105,5 (s); 166,1 (s); 101,6 (d); 163,7 (s); 112,5 (d); 144,6 (s); 24,3 (q).

SUBESTRUTURAS PROPOSTAS PELO SISCONST:
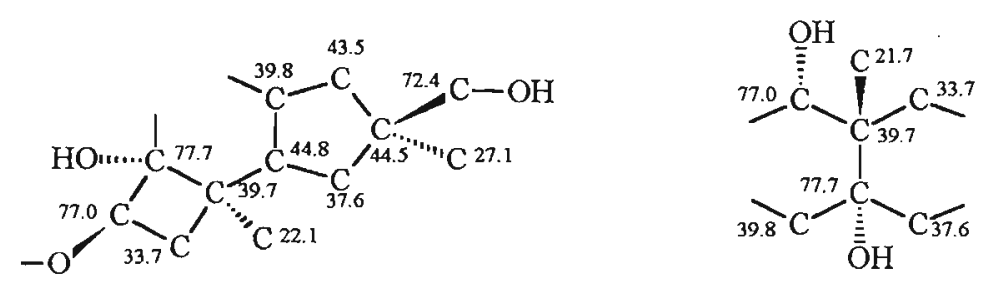

PROBABILIDADE DE ESQUELETO (\%)

\begin{tabular}{lccccc}
\hline ESQUELETO & SISCONST & C13MACH & SISOCBOT & REGRAS & GLOBAL \\
\hline \hline PROTOILUDANO & 44.7 & 32.7 & 100.0 & 100.0 & 66.2 \\
TRIFARANO & 23.1 & - & - & - & 7.4 \\
EUDESMANO & 5.8 & 10.6 & - & - & 4.4 \\
ESQ1 & - & 9.8 & - & - & 2.4 \\
CHAMIGRANO & - & 9.8 & - & - & 2.4 \\
PREZIZANO & - & 9.6 & - & - & 2.3 \\
NARDOSINANO & - & 9.2 & - & - & 2.2 \\
CARIOFILANO & - & 9.2 & - & - & 2.2 \\
\hline
\end{tabular}

SUBESQUELETO: $117[4,5 \mathrm{OR}]-100 \% ; 117[1 \mathrm{OR}]-100.0 \%$.

MACRONO: Orselinato - $172,0 \mathrm{~s} ; 105,5 \mathrm{~s} ; 166,1 \mathrm{~s} ; 101,6 \mathrm{~d} ; 163,7 \mathrm{~s} ; 112,5 \mathrm{~d} ; 144,6 \mathrm{~s} ; 24,3 \mathrm{q}$. 


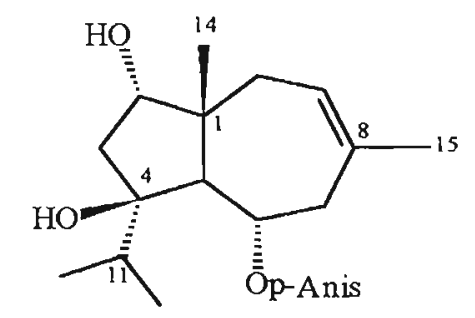

Phytochemistry, 1997, 45, 1633

Esqueleto CAROTANO

Pluchea dioscoridis (Asteraceae)

$\operatorname{RMN}^{13} \mathrm{C}\left(\mathrm{CDCl}_{3}, \mathrm{C1}-\mathrm{C15}\right): 48,2$ (s); 80,3 (d); 41,6 (t); 85,6 (s); 54,6 (d); 70,5 (d); 40,6 (d); 133,6 (s); 124,6 (d); 35,0 (t); 37,0 (d); 18,2 (q); 17,7 (q); 20,1 (q); 25,8 (q); p-Ani - 166,6 (s); 122,6 (s); 113,7 (d); $131,6(d) ; 163,5(s) ; 55,3(q)$.

SUBESTRUTURAS PROPOSTAS PELO SISCONST:
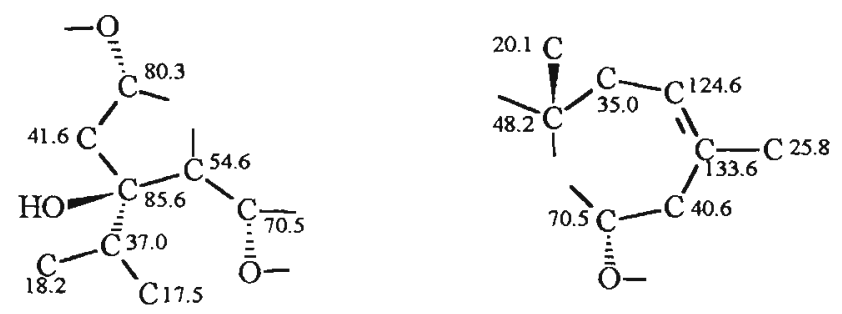

PROBABILIDADE DE ESQUELETO (\%)

\begin{tabular}{lccccc}
\hline ESQUELETO & SISCONST & C13MACH & SISOCBOT & REGRAS & GLOBAL \\
\hline \hline CAROTANO & 90.7 & 100.0 & - & 50.0 & 71.0 \\
EUDESMANO & 4.5 & - & 75.0 & 50.0 & 25.4 \\
OUTROS & 2.8 & - & - & - & 9.9 \\
GUAIANO & 2.0 & - & - & - & 9.6 \\
MONOCICLOFARNESANO & - & - & 16.7 & - & 1.3 \\
$11,12,13$ NOR-EUDESMANO & - & - & 8.3 & - & 0.7 \\
\hline
\end{tabular}

SUBESQUELETOS: $79[5,10 \mathrm{OR} ; 2 \mathrm{EN}]-100.0 \% ; 1[1,9 \mathrm{OR} ; 5,11 \mathrm{OXI}]-100.0 \%$

MACRONO : p-Anisato - $166,6 \mathrm{~s} ; 122,6 \mathrm{~s} ; 113,7 \mathrm{~d} ; 131,6 \mathrm{~d} ; 163,5 \mathrm{~s} ; 55,3 \mathrm{q}$. 


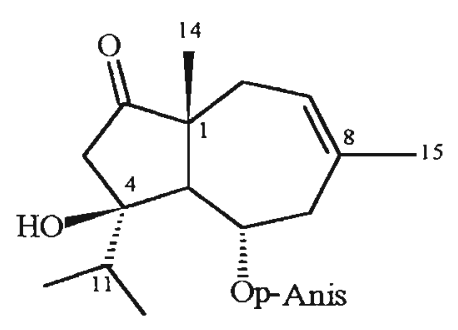

Phytochemistry, 1997, $\underline{45}, 1633$

Esqueleto CAROTANO

Pluchea dioscoridis (Asteraceae)

RMN ${ }^{13} \mathrm{C}\left(\mathrm{CDCl}_{3}, \mathbf{C 1} 1 \mathrm{C15}\right): 50,7$ (s); 219,0 (s); 44,1 (t); 80,3 (s); 55,5 (d); 69,9 (d); 41,3 (t); 133,6 (s); 123,3 (d); 35,6 (t); 36,5 (d); 18,7 (q); 17,8 (q); 19,3 (q); 26,9 (q); p-Anis - 167,2 (s); 122,3 (s); 113,9 (d); $131,8(d) ; 163,8(s) ; 56,3$ (q).

SUBESTRUTURAS PROPOSTAS PELO SISCONST:
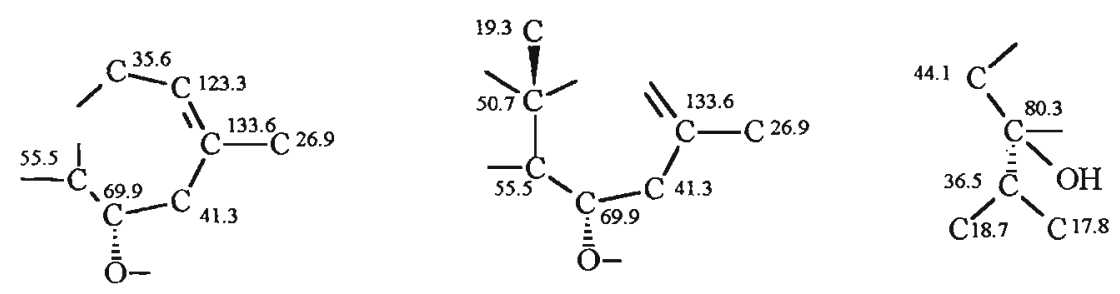

PROBABILIDADE DE ESQUELETO (\%)

\begin{tabular}{lccccc}
\hline ESQUELETO & SISCONST & C13MACH & SISOCBOT & REGRAS & GLOBAL \\
\hline \hline CAROTANO & 87.5 & 100.0 & - & 50.0 & 70.0 \\
EUDESMANO & 3.9 & - & 75.0 & 50.0 & 25.2 \\
MONOCICLOFARNESANO & 1.6 & - & 16.7 & - & 1.8 \\
OUTROS & 3.1 & - & - & - & 1.0 \\
VETISPIRANO & 2.3 & - & - & - & 0.7 \\
BOTRIANO & 1.6 & - & - & - & 0.5 \\
$11,12,13-$ TRINOR-EUDESMANO & - & - & 8.3 & - & 0.7 \\
\hline
\end{tabular}

SUBESQUELETO: $79[5,10 \mathrm{OR} ; 2 \mathrm{EN}]-100.0 \% ; 79[8 \mathrm{OXO} ; 2 \mathrm{EN}]-100.0 \% ; 1[1,9, \mathrm{OR} ; 5,11 \mathrm{OXM}]-100.0 \%$. MACRONO : p-Anisato - 167,6 s; 127,0 s; 140,3 d; 15,9 q; 20,5 q. 


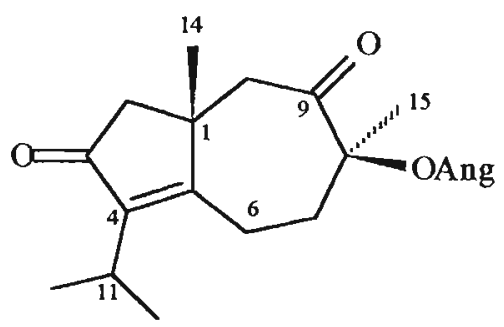

Phytochemistry, 1997, $\underline{45}, 1633$

Esqueleto CAROTANO

Pluchea dioscoridis (Asteraceae)

RMN ${ }^{13} \mathrm{C}\left(\mathrm{CDCl}_{3}, \mathrm{C1}-\mathrm{C15}\right): 41,2(\mathrm{~s}) ; 48,8(\mathrm{t}) ; 205,3$ (s); 144,0 (s); 174,6 (s); 21,1 (t); 40,4 (t); 85,5 (s); 207,2 (s); 42,8 (t); 25,3 (d); 20,1 (q); 20,5 (q); 30,1 (q), 21,4 (q); Ang - 167,6 (s); 127,0 (s); 140,3 (d); 15,9 (q); 20,5 (q).

SUBESTRUTURAS PROPOSTAS PELO SISCONST:
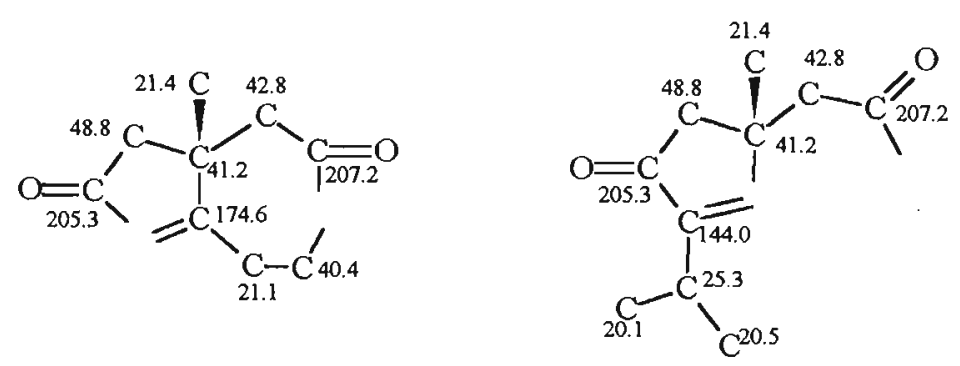

PROBABILIDADE DE ESQUELETO (\%)

\begin{tabular}{lccccc}
\hline ESQUELETO & SISCONST & C13MACH & SISOCBOT & REGRAS & GLOBAL \\
\hline \hline CAROTANO & 100.0 & 24.8 & - & 100.0 & 74.0 \\
EUDESMANO & - & 28.5 & 75.0 & - & 12.8 \\
BOTRIANO & - & 9.8 & - & - & 2.4 \\
CADINANO & - & 9.5 & - & - & 2.3 \\
EREMOFILANO & - & 9.2 & - & - & 2.2 \\
3,4SECO-13(->9)DRIMANO & - & 9.1 & - & - & 2.2 \\
TREMULANO & - & 9.0 & & - & 2.1 \\
\hline
\end{tabular}

SUBESQUELETO: $79[2 \mathrm{OXO} ; 6(10) \mathrm{EN}]-100.0 \% ; 79[6(10) \mathrm{EN}]-100.0 \%$.

MACRONO : Angelato - 167,6 s; 127,0 s; 140,3 d; 15,9 q; 20,5 q. 
TESTES REALIZADOS COM MISTURAS DE SESQUITERPENOS

\section{MISTURA 1}

COMPONENTES DA MISTURA : Espatulenol e Alismol.

Ocorrência botânica: Xylopia emarginata

RMN ${ }^{13} \mathrm{C}: 53.4 \mathrm{~d}, 24.7 \mathrm{t}, 41.7 \mathrm{t}, 81.0 \mathrm{~s}, 53.0 \mathrm{~d}, 29.9 \mathrm{~d}, 27.4 \mathrm{~d}, 26.7 \mathrm{t}, 38.8 \mathrm{t}, 153.4 \mathrm{~s}$, 20.2 s, 28.6 q, 16.3 q, 26.0 q, 106.2 t, 47.3 d, 24.8 t, 30.0 t, 81.0 s, 55.0 d, 121.3 d, $149.8 \mathrm{~s}, 37.1 \mathrm{t}, 40.2 \mathrm{t}, 153.9 \mathrm{~s}, 37.4 \mathrm{~d}, 21.3 \mathrm{q}, 21.5 \mathrm{q}, 24.1 \mathrm{q}, 106.5 \mathrm{t}$.

\section{ESTRUTURAS FORNECIDAS PELO SISCONST e C13MACH :}<smiles></smiles>

Espatulenol

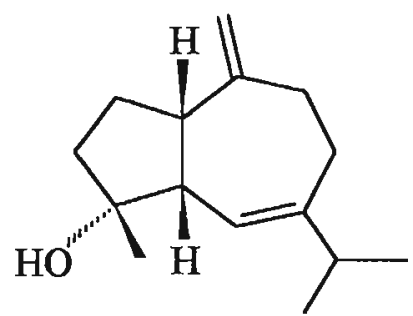

Alismol 


\section{MISTURA 2}

COMPONENTES DA MISTURA : $\alpha$-Guaieno, $\alpha$-Bulneseno, $\alpha$-Patchouleno $e$ Seicheleno (J. Essent. Oil Res., 1997, 9 , 321).

Ocorrência botânica : Pogostemon cablin (Lamiaceae).

RMN ${ }^{13} \mathrm{C}: 61.7,145.5,43.6,40.0,35.5,162.5,142.0,128.8,152.2,140.6,138.7,152.1$ (s), 26.3, 38.2, 33.8, 34.2, 119.3, 26.8, 31.9, 28.0, 26.7, 26.8, 37.5, 103.6, 30.4, 33.2, $32.9,32.0,33.8,108.1,36.3,31.3,33.4,31.2,34.0,108.0(t), 34.8,59.8,47.3,37.9$, $45.1,30.1,38.9,46.3,50.9,46.6,46.7,33.9$ (d) $21.3,21.1,15.7,25.0,20.8,18.8$, $20.9,22.2,15.4,20.4,18.6,19.8(q)$.

\section{ESTRUTURAS FORNECIDAS PELO SISCONST e C13MACH :}

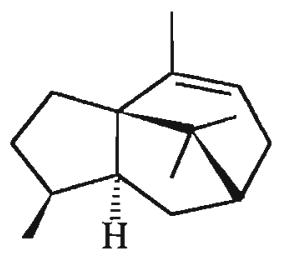

$\alpha$-pachouleno

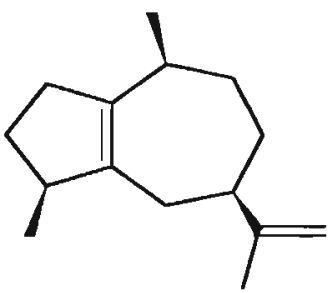

$\alpha$-guaieno

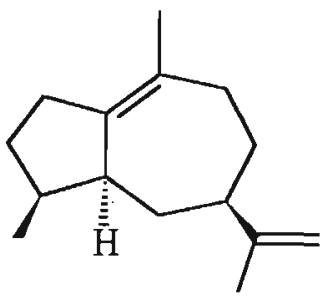

$\alpha$-bulneseno

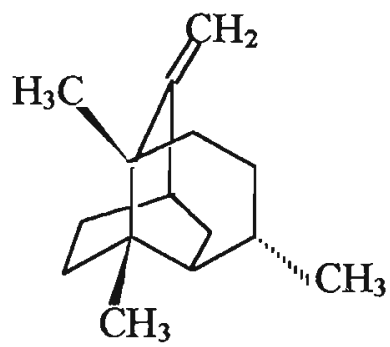

seicheleno 


\section{MISTURA 3}

COMPONENTES DA MISTURA : $\alpha$-Eudesmol, $\beta$-Eudesmol, $\lambda$-Eudesmol, Carissona, Guaiol, Bulnesol, Elemol (Magn. Res. Chem.,1995, 233).

Ocorrência botânica : Neocallitropsis Pancheri

RMN ${ }^{13} \mathrm{C}: 37.8,22.9,24.3,22.4,40.1,41.9,23.5,36.9,25.0,22.4,41.1,105.3$, $42.3,19.2,33.2,26.4,23.3,40.3,37.3,33.8,28.8,22.6,42.0,35.4,31.0,27.9,27.4$, $33.8,30.3,33.1,28.8,27.8,34.9,109.9,112.1,28.5,22.6,39.9(\mathrm{t}), 120.9,46.6,49.9$, $49.8,49.4,50.6,49.7,46.3,49.6,33.8,39.0,46.3,54.1,150.3,52.8,49.4$ (d), 135.1 , $32.1,73.0,150.9,35.8,72.7,124.5,134.9,34.5,72.9,199.2,128.8,163.0,35.9,72.4$, $140.0,139.0,73.5,141.6,128.9,73.8,147.9,39.7,72.7$ (s), 27.4, 26.7, 21.1, 15.5, $27.1,27.1,16.3,27.2,26.9,19.3,24.7,27.5,26.7,10.9,22.5,26.1,27.4,19.9,19.8$, $27.3,27.1,15.4,22.3,27.2,27.1,24.8,16.6(q)$.

ESTRUTURAS FORNECIDAS PELO SISCONST e C13MACH :<smiles>CC1=CCC[C@]2(C)CCC(C(C)(C)O)C[C@H]12</smiles>

$\alpha$-Eudesmol<smiles>CC1=C2C[C@H](C(C)(C)O)CC[C@]2(C)CCC1=O</smiles>

Carissona<smiles>C=C1CCC[C@]2(C)CCC(C(C)(C)O)C[C@@H]12</smiles>

$\beta$-Eudesmol

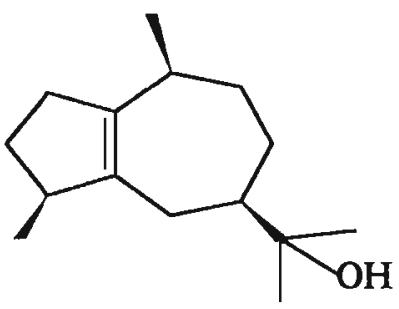

Guaiol<smiles>CC1=C2C[C@H](C(C)(C)O)CC[C@]2(C)CCC1</smiles>

$\lambda$-Eudesmol

Bulnesol<smiles>C=C[C@]1(C)CCC(C(C)(C)O)C[C@H]1C(=C)C</smiles>

Elemol 


\section{DISCUSSÃO DOS RESULTADOS}

Analisando-se os resultados dos testes anteriormente apresentados, verificamos pelos valores de probabilidade global fornecidos pelo pacote dos programas de previsão de esqueletos, que o esqueleto correto foi indicado como primeira opção em $86,7 \%$ dos casos e, se considerarmos até a quarta opção, o sistema apresentou um acerto de $96,7 \%$. Nos casos em que a probabilidade global para o esqueleto apresentou um valor baixo, menor do que $40 \%$, deve-se levar em consideração não apenas o seu valor absoluto, mas também o seu valor relativo, comparado às probabilidades de outros esqueletos, que normalmente são muito menores.

O programa SISCONST apresentou um índice de acerto de $83,3 \%$, considerando-se o esqueleto correto como primeira opção e um acerto de $95,0 \%$, quando levada em conta a segunda opção. Comparado com o programa $\mathrm{C} 13 \mathrm{MACH}$, que acertou $73,0 \%$ dos testes, o SISCONST apresentou-se mais eficiente. Isto deve-se ao fato de que o programa SISCONST seleciona para a determinação dos esqueletos mais prováveis apenas os sesquiterpenos que contenham no mínimo sete átomos de carbonos interligados, com os deslocamentos quimicos e as multiplicidades requeridas pelo usuário. Já o programa $\mathrm{C} 13 \mathrm{MACH}$ realiza apenas um confronto entre os deslocamentos químicos do espectro-problema e os dados espectrais presentes no banco de dados, sem a restrição de interligação entre os átomos que apresentam aqueles deslocamentos.

Em geral, observa-se que o programa SISCONST erra na previsão de esqueleto quando o número de substâncias presentes no banco, referentes ao tipo de esqueleto da substância analisada, é muito pequeno. A não ser que, apesar de estar pouco representado na base de dados, aquele tipo de esqueleto apresente sinais de $\operatorname{RMN}{ }^{13} \mathrm{C}$ muito característicos, ou a substância em questão tenha um espectro semelhante àqueles presentes no banco de dados. Como exemplo, podemos citar os esqueletos $\alpha$ santalano e $\beta$-santalano (testes 12 e 13), cujos números de substâncias presentes no banco de dados são, respectivamente, 11 e 1 . Nos testes de número 11 e 38 , o SISCONST não fornece o esqueleto correto entre os mais prováveis. O número de substâncias do esqueleto acorano presentes no banco é igual a 4 e pouco oxidadas. Já no caso do teste 38 , o esqueleto 2-9-cariofilano ainda não está presente no banco de dados.

Analisando as subestruturas propostas pelo SISCONST, observamos que em vários testes, a sobreposição ou a união de algumas subestruturas pode conduzir a uma subestrutura maior ou até mesmo à estrutura completa da substância, como por 
exemplo, nos testes $43,48,52,55$ e 58 . Dentre as 128 subestruturas apresentadas nos testes, $81,3 \%$ são compatíveis com as estruturas propostas na literatura. Muitas vezes, as subestruturas propostas são parecidas, pois um mesmo sinal do espectro de RMN ${ }^{13} \mathrm{C}$ analisado pode ser atribuído a diferentes átomos em diferentes subestruturas.

O programa SISCONST também pode auxiliar na tarefa de atribuição dos sinais de RMN ${ }^{13} \mathrm{C}$. Nos testes realizados, dentre os sinais atribuidos pelo programa, $81,5 \%$ foram atribuídos corretamente, de acordo com a literatura.

O programa SISOCBOT que realiza a análise dos dados botânicos, apresentou um índice de acerto de $43 \%$, o que é até bastante significativo, tendo em vista que o levantamento das ocorrências botânicas de sesquiterpenos não fazia parte do objetivo deste trabalho. Apenas foram levantados os dados botânicos dos sesquiterpenos cujos espectros de $\mathrm{RMN}{ }^{13} \mathrm{C}$ constavam na literatura. Portanto, o número de ocorrências botânicas presentes no banco é muito pequeno, comparado ao número de ocorrências existentes. Quando a substância analisada pertence a uma família não presente no banco de dados, o programa não fornece nenhuma previsão de esqueleto, porém, caso o gênero seja desconhecido ou não esteja presente no banco, a pesquisa poderá ser feita, utilizando-se o nome da familia. Nestes casos, no entanto, o resultado é menos confiável. O SISOCBOT, destaca-se, então, como mais uma ferramenta, somado aos programas SISCONST, C13MACH e REGRAS, para a previsão do tipo de esqueleto de sesquiterpenos.

O programa REGRAS, baseado nas faixas características de deslocamentos químicos de esqueletos ou subesqueletos (tabela 15) e no padrão dos tipos de carbonos para cada esqueleto desfuncionalizado (tabela 12), apresentou um índice de acerto semelhante ao do programa SISCONST, embora forneça valores de probabilidade geralmente em tomo de $100 \%$. Este programa indicou o subesqueleto correto em $68 \%$ dos casos analisados. Em alguns casos, apresenta mais de um subesqueleto, devido ao fato da substância apresentar deslocamentos químicos característicos de ambos, por exemplo, no teste 43, a substância-teste tem o esqueleto cariofilano e apresenta os grupos funcionais característicos dos dois subesqueletos, 0 8(14)-en-cariofilano e o 4,5-epóxi-cariofilano.

Nos casos em que não há regras para o esqueleto ou subesqueleto, o programa REGRAS pode indicar quais são os esqueletos possíveis de acordo com o número de sinais do espectro de $\mathrm{RMN}{ }^{13} \mathrm{C}$ e o número de carbonos quaternários, metínicos, metilênicos e metílicos do esqueleto desfuncionalizado. Por exemplo, a substância do teste 17, apresenta 14 átomos de carbono, sendo 1 quatemário, $5 \mathrm{CH}, 5 \mathrm{CH}_{2}$ e $3 \mathrm{CH}_{3}$. Com estas características, existe apenas um único esqueleto possivel para tal substância, que é o 15-Nor-iludano. O grupo glicosila presente na substância foi 
identificado pelo programa MACRONO, sendo os seus deslocamentos químicos retirados do espectro antes da análise pelos programas de previsão de esqueleto.

Os resultados de alguns dos testes com o programa REGRAS revelaram um erro cometido pelo programa durante a análise das faixas de deslocamentos químicos de ${ }^{13} \mathrm{C}$. No teste 11 , por exemplo, o programa forneceu uma probabilidade de $50,0 \%$ para o esqueleto $\alpha$-santalano e $50,0 \%$ para o subesqueleto 2(4)en-protoiludano. No entanto, as faixas de deslocamentos químicos obtidas para o subesqueleto 2(4)-enprotoiludano apresentam dois singletos na região de 52,2-34,7 e 47,5-38,5 $\delta$; enquanto - espectro questionado tem apenas um único singleto em $44,9 \delta$ que pode ser associado a ambas as faixas. Sendo assim, o programa deveria ter fomecido apenas o esqueleto $\alpha$-santalano, pois os deslocamentos químicos do espectro analisado, que são característicos deste esqueleto $(38,4 d ; 44,9 s ; 27,4 s)$, estão correlacionados com as faixas de deslocamentos obtidas para o mesmo, 40,0-37,0d; 48,0-44,0s e 29,0-25,0s, segundo uma relação unívoca. Deste modo, será necessária uma modificação no programa para que ele não associe um mesmo deslocamento químico do espectro analisado a diferentes faixas de deslocamentos químicos obtidas para um determinado esqueleto. O mesmo tipo de erro também ocorreu na análise das substâncias dos testes 32,33 e 40 .

O programa MACRONO foi utilizado em 26 testes, identificando com sucesso $97 \%$ dos grupos substituintes, entre eles, glicosila, isobutirila, p-anisoíla, orselinoíla, acetila, angeloila, 2-metilbutirila e metóxila. Em certos casos, observamos que o programa atribuiu ao substituinte um ou dois sinais do espectro que pertenciam, de acordo com a literatura, a carbonos do esqueleto da substância. Isto deve-se ao fato de que aqueles deslocamentos estavam mais próximos dos deslocamentos do macronó presente no banco de dados. Nos testes aqui apresentados isto não prejudicou a previsão de esqueleto ou a obtenção de subestruturas, mas sim a atribuição dos sinais do espectro questionado. Em outros casos, porém isto pode determinar um erro na análise posteriormente realizada pelos programas SISCONST, C13MACH e REGRAS. Para evitar este tipo de erro e também a proposta de outros macronós que não correspondam àqueles presentes na substância analisada, mas que podem aparecer nos resultados, devido à necessidade de se aumentar o gradiente na pesquisa do macronó, pretende-se armazenar dados de deslocamentos químicos dos diversos macronós em vários solventes, de modo que a pesquisa possa ser realizada de acordo com o solvente no qual foi obtido o espectro de ${ }^{13} \mathrm{C}$ da amostra. Muitas vezes, o efeito do solvente pode conduzir a uma grande diferença entre os deslocamentos químicos do 
macronó presente no banco de dados e aqueles do macronó da substância analisada em outro solvente, atingindo valores de $6 \delta$, como é o caso dos açúcares.

Os testes realizados para análise de misturas de sesquiterpenos, utilizando-se os programas SISCONST e C13MACH, também apresentaram resultados muito bons e animadores, pois foi possível a identificação dos componentes de três frações de diferentes óleos essencias, contendo um número de 2, 4 e 7 sesquiterpenos. Porém, os programas que podem fazer este tipo de análise permitem a entrada de um número máximo de apenas 60 deslocamentos químicos com as respectivas multiplicidades. Para o teste com a mistura de sete sesquiterpenos, foram retirados os sinais de algumas metilas e outros que eram muito próximos e tinham a mesma multiplicidade. $\grave{A}$ medida que o programa identificava um dos componentes, os sinais atribuídos a ele eram retirados e aqueles deslocamentos inicialmente descartados eram incluídos ao espectro para nova análise. Este processo foi repetido até a identificação de todos os componentes da amostra, porém não é nada prático e torna-se inviável para uma mistura com número de compostos maior do que sete, podendo conduzir também a falsos resultados, dependendo da combinação que foi feita para os sessenta primeiros sinais a serem analisados. Portanto, deverão ser realizadas modificações nos programas SISCONST e C13MACH, de modo que eles permitam a entrada de um número de deslocamentos químicos superior a 150, para que se possam analisar misturas de pelo menos dez componentes. 


\section{CONCLUSÃO}

O SISTEMAT é um sistema especialista que foi criado com o objetivo principal de auxiliar os espectroscopistas no processo de determinação estrutural, especificamente, de produtos naturais. Este sistema ainda se encontra em fase de desenvolvimento e aperfeiçoamento, não sendo realizada, até o momento, a geração automática de estruturas completas, pois o programa gerador desenvolvido requer algumas modificações para o seu bom funcionamento. No entanto, os programas aplicativos que compõem o SISTEMAT tem sido consideravelmente testados para a previsão do tipo de esqueleto, com compostos tais como diterpóides, triterpenóides, monoterpenóides e sesquiterpenos lactonizados, apresentando bons resultados.

As análises realizadas neste trabalho também mostram um bom desempenho dos programas do SISTEMAT para a previsão do tipo de esqueleto de sesquiterpenos, com um acerto médio de $84 \%$. Isto já demonstra o papel fundamental que este sistema pode desempenhar, no sentido de aumentar a capacidade de interpretação espectral dos pesquisadores, tendo em vista que a caracterização do tipo de esqueleto de um produto natural desconhecido é um ponto-chave na determinação estrutural destes compostos.

O programa SISCONST, juntamente com os esqueletos mais prováveis para uma dada substância, fornece também grandes subestruturas com sinais de ${ }^{13} \mathrm{C}$ atribuídos, cuja sobreposição ou união, muitas vezes pode conduzir à estrutura completa de um composto ou pelo menos a estruturas maiores relevantes para o estabelecimento de uma proposta estrutural.

No SISTEMAT, além das informações espectrais de RMN ${ }^{13} \mathrm{C}$, também são utilizados dados botânicos como mais uma ferramenta para previsão do tipo de esqueleto do composto isolado, pois observa-se que algumas famílias tendem a produzir metabólitos secundários com um determinado tipo de esqueleto.

As informações obtidas a partir dos programas aplicativos, tais como os prováveis tipos de esqueletos para um composto analisado, as grandes subestruturas, - percentual de reconhecimento de subesqueletos e os prováveis grupos substituintes presentes na estrutura, serão utilizadas como grandes restrições pelo programa gerador de estrutura, de modo a se obter um número menor de propostas estruturais e uma redução do tempo de análise computacional. Nenhum outro sistema inicia o processo de geração de estrutura a partir do provável esqueleto da substância.

Os testes realizados com os programas SISCONST e C13MACH para a identificação de sesquiterpenos em misturas, como por exemplo, óleos essenciais, 
apresentaram ótimos resultados, sendo necessária apenas uma modificação nesses programas para que eles permitam a entrada de um grande número de deslocamentos químicos e multiplicidades.

Algumas das modificações necessárias em outros programas já foram discutidas anteriormente no item discussão dos resultados. Outros programas devem ser desenvolvidos como, por exemplo, um programa que obtenha automaticamente as faixas de deslocamentos químicos característicos para esqueletos ou subesqueletos. 0 programa deveria ser capaz de escolher os átomos cujas faixas de deslocamentos químicos devem ser analisadas, o que hoje está sendo feito pelos químicos, e determiná-las para posterior verificação do percentual de reconhecimento, confrontando estas faixas com dados de $\mathrm{RMN}^{13} \mathrm{C}$ de todos os compostos do banco de dados. Este programa é muito importante para o SISTEMAT, pois, à medida que aumenta o número de compostos no banco de dados, as faixas características de deslocamentos químicos precisam ser revistas e atualizadas, tendo em vista que, podem ter sido ampliadas ou terem sofrido alterações nos seus percentuais de reconhecimento. Além disso, novas regras heurísticas podem ser obtidas. 


\section{REFERÊNCIAS BIBLIOGRÁFICAS}

1. E. Rich, "Inteligência Artificial" ; São Paulo, McGraw-Hill, (1988).

2. S. M. Weiss, C. A. Kulikowski, "Guia Prático Para Projetar Sistemas Especialistas" ; Rio de Janeiro, Livros Técnicos e Científicos, (1988).

3. H. M. Cartwright, "Applications of Artificial Intelligence in Chemistry" ; New York, Oxford University Press Inc., (1993).

4. Z. Hippe, "Artificial Intelligence in Chemistry - Structure elucidation and simulation of organic reactions" ; Warszawa, Polish Scientific Publishers, (1991).

5. E. H. Shortliffe, "Computer-based medical consultations: MYCIN." ; New York, Elsevier, (1976).

6. R. O. Duda, P. E. Hart, K. Konolige, R. Reboh, "A Computer based consultant for mineral exploration"; Technical Report, SRI International, (1979).

7. T. D. Salatin, Jorgensen, W. L. Jorgensen, "Computer assisted mechanistic evaluation of organic reactions. 1. Overview"; J. Org. Chem., 45, 2043 (1980).

8. N. A. B. Gray, "Computer-assisted structure elucidation"; New York, John Willey \& Sons, (1986).

9. R. Attias, "DARC Substructure Search System: A New Approach to Chemical Information"; J. Chem. Inf. Comp. Sc., 23, 102 (1983).

10. W. Bremser, M. Grzonka, "Specinfo - A Multidimensional Spectroscopic Interpretation System"; Mikrochim. Acta, II, 483 (1991).

11. R. K. Lindsay, B. G. Buchanan, E. A. Fergenbaum, J. Lederberg, "Applications of Artificial Intelligence for Organic Chemistry: the DENDRAL project;; New York, McGraw-Hill, (1980). 
12. C. Djerassi, D. H. Smith, C. W. Crandell, N. A. B. Gray, J. G. Nourse, M. R. Lindley, "The DENDRAL Project: computational aids to natural products structure elucidation"; Pure and Appl. Chem., 54, 2425 (1982).

13. I. Kubo, Y. M. Lee, M. Pettei, F. Pilkiewicz, K. Nakanishi, "Potent army worm antifeedants from the east african warburgia plants"; J. Chem. Soc. Chem. Commun., 1013 (1976).

14. R. Neudert, M. Penk, "Enhanced Structure Elucidation"; J. Chem. Inf. Comput. Sci., 36, 244 (1996).

15. W. Bremser, "HOSE- A novel substructure code"; Analytica Chimica Acta, 103, 355 (1978).

16. M. Will, W. Fachinger, J. R. Richert, "Fully Automated Structure Elucidation - A Spectroscopist's Dream Comes True"; J. Chem. Inf. Comput. Sci., 36, 221 (1996).

17. M. J. Pestchanker, M. S. Ascheri, O. S. Giordano; Phytochemistry, 24(7), 1622 (1985).

18. T. Konoshima; M. Takasaki, M. Kozuka, M. Haruna, K. Ito, J. R. Estes, K. Lee, "Constituents of Rosaceous Plants. I. Structures of New Triterpenoids from Cowania mexicana"; Chem. Pharm. Bull., 41(9), 1612 (1993).

19. J. P. Gastmans, M. Furlan, M. N. Lopes, J. H. G. Borges, V. P. Emerenciano, "A Inteligência Artificial Aplicada à Química de Produtos Naturais. O programa SISTEMAT. Parte I - Bases Teóricas"; Quím. Nova, 13(1), 10 (1990).

20. J. P. Gastmans, M. Furlan, M. N. Lopes, J. H. G. Borges, V. P. Emerenciano, "A Inteligência Artificial Aplicada à Química de Produtos Naturais. O programa SISTEMAT. Parte II - Organização do Programa e Aplicativos"; Quím. Nova, 13(2), 75 (1990).

21. J. P. Gastmans, M. N. Lopes, M. Furlan, V. P. Emerenciano; Comput. and Chem., 14, 75 (1990). 
22. J. P. Gastmans, J. C. Zurita, J. Sahão, V. P. Emerenciano; Anal. Chem. Acta, 217, 85 (1989).

23. D. L. G. Fromanteau, J. P. Gastmans, S. A. Vestri, V. P. Emerenciano, J. H. G. Borges; Comput. and Chem., 17, 369 (1993).

24. S. A. V. Alvarenga, "Criação de um Sistema Especialista em diterpenóides com base em RMN ${ }^{13} \mathrm{C}$ e dados botânicos". Tese de doutorado, IQ-USP, São Paulo (1993).

25. P. A. T. Macari, "Desenvolvimento de Novas Técnicas de Inteligência Artificial para a Identificação de Esqueletos de Triterpenos". Tese de doutorado, IQ-USP, São Paulo (1994).

26. V. P. Emerenciano, G. V. Rodrigues, J. P. Gastmans, "Um método de codificação semi-automático assistido por computador para o SISTEMAT", Quím. Nova, 15, 431 (1993).

27. M. J. P. Ferreira, V. P. Emerenciano, G. A. R. Lima, P. Romoff, P. A. T. Macari, G. V. Rodrigues, ${ }^{~}{ }^{13} \mathrm{C}$ NMR Spectroscopy of Monoterpenoids"; Progress in Nuclear Magnetic Ressonance, no prelo.

28. G. V. Rodrigues, "Um Sistema Especialista para Determinação Estrutural de Sesquiterpenos Lactonizados - um enfoque multi-espectral aliado a dados botânicos". Tese de doutorado, IQ-USP, São Paulo (1996).

29. V. P. Emerenciano, A. C. Bussolini, G. V. Rodrigues, "A pattern recognition method on ${ }^{13} \mathrm{C}$ NMR spectroscopy of sesquiterpenes skeletal types"; Spectroscopy, 11, 95 (1993).

30. A. P. Lins, M. Furlan, J. P. Gastmans e V. P. Emerenciano, "Aplicações de Técnicas de Inteligência Artificial em Química Orgánica. Estudo, por Computador, de RMN ${ }^{13} \mathrm{C}$ de Esteróides"; An. Acad. bras. Ci., 63(2), 141 (1991).

31. V. P. Emerenciano, L. D. Melo, G. V. Rodrigues, J. P. Gastmans, "Application of Artificial Intelligence in Organic Chemistry. Part XIX. Pattern recognition and 
strutuctural determination of flavonoids using ${ }^{13}$ C-NMR spetra"; Spectroscopy, 13 , 181(1997).

32. J. H. G. Borges, "Design e Implementação de um Gerador Estrutural Automático para o Projeto SISTEMAT". Tese de doutorado, IQ-USP, São Paulo (1998).

33. M. Carabedian, I. Dagane, J. E. Dubois, "Elucidation by progressive intersection of ordered substructures from carbon-13 nuclear magnetic resonance", Anal. Chem., 60, 2186 (1988).

34. D. L. G. Fromanteau, J. P. Gastmans, S. A. Vestri, V. P. Emerenciano, J. H. G. Borges, "A constraints generator in structural determination by microcomputer"; Computers Chem. , 17(4), 369 (1993).

35. J. A. Marco, J. F. Sanz-Cervera, M. D. Morante, V. G. Lliso, Joan V. Xirau, J. Jakupovic, Phytochemistry, 41(3), 837 (1996).

36. W. Bremser, M. Klier, E. Meyer; Org. Magn. Res., 7, 97 (1975).

37. J. P. Gastmans, V. P. Emerenciano, Computer and Chemistry, 12, 285 (1988).

38. G. V. Rodrigues, I. P. A. Campos , V. P. Emerenciano, "Applications of artificial intelligence to structure determination of organic compounds. $X X$. Determination of groups attached to the skeleton of natural products using ${ }^{13} \mathrm{C}$ nuclear magnetic resonance spectroscopy", Spectroscopy, 13, 191 (1997). 